\author{
Ph.D. Dissertation
}

\title{
Transmit and Receive Signal Processing for MIMO Terrestrial Broadcast Systems
}

\author{
Universitat Politècnica de València \\ Departamento de Comunicaciones
}

\author{
Author \\ David E. Vargas Paredero \\ Advisor \\ Dr. David Gómez-Barquero \\ Tutor \\ Prof. Narcís Cardona Marcet
}

Valencia, May 2016 



\section{Supervisors}

Dr. David Gómez-Barquero

Institute of Telecommunications and Multimedia Applications

Universitat Politècnica de València, Spain

Professor Narcís Cardona Marcet

Communications Department

Universitat Politècnica de València, Spain

\section{Reviewers}

Associate Professor Camilla Hollanti

Department of Mathematics and Systems Analysis

Aalto University, Finland

Associate Professor Jeongchang Kim

Division of Electronics and Electrical Information Engineering

Korea Maritime and Ocean University, South Korea

Assistant Professor Youssef Nasser

Faculty of Engineering and Architecture

American University of Beirut, Lebanon

\section{Opponents}

Professor Alberto González Salvador

Communications Department

Universitat Politècnica de València, Spain

Dr. Mikel Mendicute Errasti

Department of Electronics and Computer Science

Mondragon Unibertsitatea, Spain

Dr. Belkacem Mouhouche

Samsung Electronics Research Institute, United Kingdom 


\section{Abstract}

Multiple-Input Multiple-Output (MIMO) technology in Digital Terrestrial Television (DTT) networks has the potential to increase the spectral efficiency and improve network coverage to cope with the competition of limited spectrum use (e.g., assignment of digital dividend and spectrum demands of mobile broadband), the appearance of new high data rate services (e.g., ultra-high definition TV - UHDTV), and the ubiquity of the content (e.g., fixed, portable, and mobile). It is widely recognised that MIMO can provide multiple benefits such as additional receive power due to array gain, higher resilience against signal outages due to spatial diversity, and higher data rates due to the spatial multiplexing gain of the MIMO channel. These benefits can be achieved without additional transmit power nor additional bandwidth, but normally come at the expense of a higher system complexity at the transmitter and receiver ends. The final system performance gains due to the use of MIMO directly depend on physical characteristics of the propagation environment such as spatial correlation, antenna orientation, and/or power imbalances experienced at the transmit aerials. Additionally, due to complexity constraints and finite-precision arithmetic at the receivers, it is crucial for the overall system performance to carefully design specific signal processing algorithms.

This dissertation focuses on transmit and received signal processing for DTT systems using MIMO-BICM (Bit-Interleaved Coded Modulation) without feedback channel to the transmitter from the receiver terminals. At the transmitter side, this thesis presents investigations on MIMO precoding in DTT systems to overcome system degradations due to different channel conditions. At the receiver side, the focus is given on design and evaluation of practical MIMOBICM receivers based on quantized information and its impact in both the in-chip memory size and system performance. These investigations are carried within the standardization process of DVB-NGH (Digital Video Broadcasting - Next Generation Handheld) the handheld evolution of DVB-T2 (Terrestrial - Second Generation), and ATSC 3.0 (Advanced Television Systems Committee - Third Generation), which incorporate MIMO-BICM as key technology to overcome the Shannon limit of single antenna communications. Nonetheless, 


\section{ABSTRACT}

this dissertation employs a generic approach in the design, analysis and evaluations, hence, the results and ideas can be applied to other wireless broadcast communication systems using MIMO-BICM.

The first part of the thesis analyses the performance and structure of MIMO precoders based on rotation matrices for $2 \times 2 \mathrm{MIMO}$ and focus on the case of cross-polar antennas, which is the preferred configuration in DTT systems in the UHF band. Analysis and evaluation with the information-theoretic limits of BICM systems and bit-error-rate simulations including channel coding show the interesting results that the performance of the precoder depends on the selected code-rate. While rotation can provide significant improvements when connected with high code-rates, the performance improvement diminishes with lower code-rates. The results obtained in this part of the dissertation provide new insights on the performance of these type precoders in typical DTT scenarios and the dependences with channel characteristics and system parameters. Furthermore, a channel-precoder is proposed that exploits statistical information of the MIMO channel. The performance of the channel-precoder is evaluated in a wide set of channel scenarios and mismatched channel conditions, a typical situation in the broadcast set-up. Capacity results show performance improvements in the case of strong line-of-sight scenarios with correlated antenna components and resilience against mismatched condition. Finally, bit-error-rate simulation results compare the performance of singleinput single-output, $2 \times 2$ and $4 \times 2$ MIMO systems and the proposed MIMO channel-precoder.

The second part of the thesis is devoted to investigations of memory and performance trade-offs of soft-quantized information in MIMO receivers. DTT systems rely on time-interleaving techniques to overcome signal fluctuations and improve the system performance. Yet, time-interleaving imposes the highest in-chip memory requirements which depend on the quantization resolution and algorithms employed at the receiver terminal. Since on-chip memory accounts for a large fraction of the chip area, it is desirable to have small word length with reduced performance loss. Two types of quantized receivers are investigated: quantization of In-phase and Quadrature (I\&Q) samples and quantization of log-likelihood ratios. The implications on the in-chip memory and the possibility of implementing MIMO-BICM with iterative decoding are presented and discussed. The performance of uniform quantization and nonuniform quantization is evaluated showing potential benefits for non-uniform quantization adapted to the signal statistics. The results obtained in this chapter provide new insights on the important trade-off between in-chip memory and system performance for receiver architectures with quantized information. 


\section{Resumen}

La tecnología de múltiples entradas y múltiples salidas (MIMO) en redes de Televisión Digital Terrestre (TDT) tiene el potencial de incrementar la eficiencia espectral y mejorar la cobertura de red para afrontar las demandas de uso del escaso espectro electromagnético (e.g., designación del dividendo digital y la demanda de espectro por parte de las redes de comunicaciones móviles), la aparición de nuevos contenidos de alta tasa de datos (e.g., ultra-high definition TV - UHDTV) y la ubicuidad del contenido (e.g., fijo, portable y móvil). Es ampliamente reconocido que MIMO puede proporcionar múltiples beneficios como: potencia recibida adicional gracias a las ganancias de array, mayor robustez contra desvanecimientos de la señal gracias a la diversidad espacial y mayores tasas de transmisión gracias a la ganancia por multiplexado del canal MIMO. Estos beneficios se pueden conseguir sin incrementar la potencia transmitida ni el ancho de banda, pero normalmente se obtienen a expensas de una mayor complejidad del sistema tanto en el transmisor como en el receptor. Las ganancias de rendimiento finales debido al uso de MIMO dependen directamente de las características físicas del entorno de propagación como: la correlación entre los canales espaciales, la orientación de las antenas y/o los desbalances de potencia sufridos en las antenas transmisoras. Adicionalmente, debido a restricciones en la complejidad y aritmética de precisión finita en los receptores, es fundamental para el rendimiento global del sistema un diseño cuidadoso de algoritmos específicos de procesado de señal.

Esta tesis doctoral se centra en el procesado de señal, tanto en el transmisor como en el receptor, para sistemas TDT que implementan MIMO-BICM (Bit-Interleaved Coded Modulation) sin canal de retorno hacia el transmisor desde los receptores. En el transmisor esta tesis presenta investigaciones en precoding MIMO en sistemas TDT para superar las degradaciones del sistema debidas a diferentes condiciones del canal. En el receptor se presta especial atención al diseño y evaluación de receptores prácticos MIMO-BICM basados en información cuantificada y a su impacto tanto en la memoria del chip como en el rendimiento del sistema. Estas investigaciones se llevan a cabo en el contexto de estandarización de DVB-NGH (Digital Video Broadcasting - Next 


\section{RESUMEN}

Generation Handheld), la evolución portátil de DVB-T2 (Second Generation Terrestrial), y ATSC 3.0 (Advanced Television Systems Commitee - Third Generation) que incorporan MIMO-BICM como clave tecnológica para superar el límite de Shannon para comunicaciones con una única antena. No obstante, esta tesis doctoral emplea un método genérico tanto para el diseño, análisis y evaluación, por lo que los resultados e ideas pueden ser aplicados a otros sistemas de comunicación inalámbricos que empleen MIMO-BICM.

La primera parte de la tesis analiza el rendimiento y la estructura de los precoders MIMO basados en matrices de rotación para MIMO $2 \times 2$ y se centra en el caso de antenas crospolarizadas que es la configuración preferida de los sistemas TDT en la banda UHF. El análisis y la evaluación con los límites de teoría de la información de los sistemas BICM y las simulaciones de bit-errorrate (BER) incluyendo codificación de canal, demuestran que el rendimiento del precoder depende del code-rate seleccionado. Mientras que la rotación puede proporcionar mejoras significativas con el uso de code-rates altos, la mejora de rendimiento se reduce utilizando code-rates bajos. Además, se propone un precoder de canal que explota la información estadística del canal MIMO. El rendimiento del precoder de canal se evalúa en una amplia variedad de escenarios de canal y en condiciones de mismatch (i.e., difieren los estadísticos usados por el precoder y los estadísticos del canal), una situación típica en los sistemas de difusión. Los resultados de capacidad demuestran mejoras de rendimiento en casos de una fuerte componente de visión directa con el transmisor junto a canales espaciales correlados, y robustez en condiciones de mismatch. Finalmente, se comparan el rendimiento mediante resultados de simulación de BER de sistemas de una antenna, $2 \times 2$ y $4 \times 2$ MIMO y el precoder de canal MIMO.

La segunda parte de la tesis investiga los trade-off entre la memoria y el rendimiento de receptores MIMO con información soft cuantificada. Los sistemas TDT utilizan técnicas de entrelazado temporal para superar las fluctuaciones de la señal y mejorar el rendimiento del sistema. Sin embargo, el entrelazado temporal impone los requisitos más altos de memoria de chip que dependen de la resolución de cuantificación y de los algoritmos en los receptores. Debido a que la memoria de chip conlleva una gran parte del área del chip, es deseable tener representaciones de palabra reducidas con una pérdida de rendimiento limitada. Dos tipos de receptores cuantificados son investigados: cuantificación de muestras In-phase y Quadrature (I\&Q) y de loglikelihood ratios. Las implicaciones en la memoria de chip y la posibilidad de implementar MIMO-BICM con decodificación iterativa se presentan y discuten. El rendimiento de cuantificación uniforme y no-uniforme es evaluado demostrando beneficios potenciales para cuantificación no-uniforme adaptada a los estadísticos de la señal. Los resultados obtenidos en este capítulo mejoran el conocimiento sobre el importante trade-off entre memoria de chip y el rendimiento para arquitecturas de recepción con información cuantificada. 


\section{Resum}

La tecnologia de múltiples entrades i múltiples eixides (MIMO) en xarxes de Televisió Digital Terrestre (TDT) té el potencial d'incrementar l'eficiència espectral i millorar la cobertura de xarxa per a afrontar les demandes d'ús de l'escàs espectre electromagnètic (e.g., designació del dividend digital i la demanda d'espectre per part de les xarxes de comunicacions mòbils), l'aparició de nous continguts d'alta taxa de dades (e.g., ultra-high deffinition TV - UHDTV) i la ubiqüitat del contingut (e.g., fix, portàtil i mòbil). És àmpliament reconegut que MIMO pot proporcionar múltiples beneficis com: potència rebuda addicional gràcies als guanys de array, major robustesa contra esvaments del senyal gràcies a la diversitat espacial i majors taxes de transmissió gràcies al guany per multiplexat del canal MIMO. Aquests beneficis es poden aconseguir sense incrementar la potència transmesa ni l'ample de banda, però normalment s'obtenen a costa d'una major complexitat del sistema tant en el transmissor com en el receptor. Els guanys de rendiment finals a causa de l'ús de MIMO depenen directament de les característiques físiques de l'entorn de propagació com: la correlació entre els canals espacials, l'orientació de les antenes, i/o els desequilibris de potència patits en les antenes transmissores. Addicionalment, a causa de restriccions en la complexitat i aritmètica de precisió finita en els receptors, és fonamental per al rendiment global del sistema un disseny acurat d'algorismes específics de processament de senyal.

Aquesta tesi doctoral se centra en el processament de senyal tant en el transmissor com en el receptor per a sistemes TDT que implementen MIMO-BICM (Bit-Interleaved Coded Modulation) sense canal de tornada cap al transmissor des dels receptors. En el transmissor aquesta tesi presenta recerques en precoding MIMO en sistemes TDT per a superar les degradacions del sistema degudes a diferents condicions del canal. En el receptor es presta especial atenció al disseny i avaluació de receptors pràctics MIMO-BICM basats en informació quantificada i al seu impacte tant en la memòria del xip com en el rendiment del sistema. Aquestes recerques es duen a terme en el context d'estandardització de DVB-NGH (Digital Video Broadcasting - Next Generation Handheld), l'evolució portàtil de DVB-T2 (Second Generation Terrestrial), 
i ATSC 3.0 (Advanced Television Systems Commitee - Third Generation) que incorporen MIMO-BICM com a clau tecnològica per a superar el límit de Shannon per a comunicacions amb una única antena. No obstant açò, aquesta tesi doctoral empra un mètode genèric tant per al disseny, anàlisi i avaluació, per la qual cosa els resultats i idees poden ser aplicats a altres sistemes de comunicació sense fils que empren MIMO-BICM.

La primera part de la tesi analitza el rendiment i l'estructura dels precoders MIMO basats en matrius de rotació per a MIMO $2 \times 2$ i se centra en el cas d'antenes amb polarització croada que és la configuració preferida dels sistemes TDT en la banda UHF. L'anàlisi i l'avaluació amb els límits de teoria de la informació dels sistemes BICM i les simulacions de bit-error-rate incloent codificació de canal, demostren els interessants resultats que el rendiment del precoder depèn del code-rate seleccionat. Mentre que la rotació pot proporcionar millores significatives mitjançant l'ús de code-rates alts, la millora de rendiment es redueix utilitzant code-rates més baixos. A més, es proposa un precoder de canal que explota la informació estadística del canal MIMO. El rendiment del precoder de canal s'avalua en una àmplia varietat d'escenaris de canal i en condicions de mismatch (i.e., difereixen els estadístics usats pel precoder i els estadístics del canal), una situació típica en els sistemes de difusió. Els resultats de capacitat demostren millores de rendiment en casos d'una forta component de visió directa amb el transmissor al costat de canals espacials correlats, i robustesa en condicions de mismatch. Finalment, es comparen el rendiment amb resultats de simulació de bit-error-rate de sistemes single-input single-output, $2 \times 2$ i $4 \times 2$ MIMO i el precoder de canal MIMO proposat.

La segona part de la tesi es dedica a recerques dels trade-off entre la memòria i el rendiment de receptors MIMO amb informació soft quantificada. Els sistemes TDT depenen de les tècniques d'entrellaçat temporal per a superar les fluctuacions del senyal i millorar el rendiment del sistema. No obstant açò, l'entrellaçat temporal imposa els requisits més alts de memòria de xip que depenen de la resolució de quantificació i dels algorismes emprats en els receptors. A causa que la memòria de xip comporta una gran part de l'àrea del xip, és desitjable tenir representacions de paraula redudes amb una pèrdua de rendiment limitada. Dos tipus de receptors quantificats són investigats: quantificació de mostres In-phase i Quadrature (I\&Q) i quantificació de log-likelihood ràtios. Les implicacions en la memòria de xip i la possibilitat d'implementar MIMOBICM amb descodificació iterativa es presenten i discuteixen. El rendiment de quantificació uniforme i no-uniforme és avaluat demostrant beneficis potencials per a quantificació no-uniforme adaptada als estadístics del senyal. Els resultats obtinguts en aquest capítol milloren el coneixement sobre l'important trade-off entre memòria de xip i el rendiment de sistema per a arquitectures de recepció amb informació quantificada. 


\section{Acknowledgements}

The realization of this Ph.D. dissertation has been a very fulfilling and rewarding experience. I would like to take this opportunity to thank all the exceptional people I have encountered through this exciting journey.

Foremost, I would like to express gratitude to my thesis advisers. Dr. David Gómez-Barquero for his excellent supervision, guidance, and constant advice over the last years, which have been very important for my professional development. Prof. Narcís Cardona Marcet for all his support during the course of this dissertation and for giving me the opportunity to pursuit my $\mathrm{Ph} . \mathrm{D}$. within his research group.

I am also very grateful to Prof. Camilla Hollanti from Aalto University, Prof. Jeongchang Kim from Korea Maritime and Ocean University, and Prof. Youssef Nasser from American University of Beirut, for acting as reviewers of this dissertation and for providing useful comments and suggestions. I would also like extend my gratitude to the thesis examiners, Prof. Alberto González Salvador from Universitat Politècnica de València, Dr. Mikel Mendicute Errasti from Mondragon Unibertsitatea and Dr. Belkacem Mouhouche from Samsung Electronics Research Institute, for taking the time to review this thesis.

During the course of this Ph.D. I had the pleasure to visit other research centres and cooperate with very talented researchers. These appointments have been a source of inspiration, learning, and personal growth.

I would like to thank Prof. Gerald Matz from the Vienna University of Technology for inviting me to his research group. His research group is a very exciting place to conduct research. A special mention goes to Dr. Andreas Winkelbauer for his excellent technical suggestions, advice and discussions from which I have learnt a lot. I would also like to thank him for providing his implementation of the Information-Bottleneck and Lloyd-Max algorithms. During my second visit, I extend my gratitude to Michael Meidlinger for his cooperation and technical suggestions.

I thank Prof. Jan Bajcsy from McGill University for hosting me at his research group. I also thank him for allowing me attend one of his courses 


\section{ACKNOWLEDGEMENTS}

from which I obtained a deeper understanding of the theoretical aspects of communication systems. During this visit I am very grateful to Prof. Yong Jin Daniel Kim for all the very interesting discussions, cooperation and for his technical advice.

I owe my deepest gratitude to Peter Moss formerly of BBC Research and Development for his supervision during my internship at BBC Research and Development. Discussing different technical topics with Peter has been a privilege and I feel very lucky to have collaborated with such a talented researcher with extraordinary motivation, great intuition, and great technical insight. His advice has not only been important for certain parts of this thesis but also for my professional development. Thank you Peter! I would also like to thank Chris Nokes for allowing me to conduct my internship within his section.

I am very grateful to all the members of the Mobile Communications Group (MCG) of the Instituto de Comunicaciones y Aplicaciones Multimedia (iTEAM) for providing such a pleasant environment: Jordi Joan Giménez, Carlos Herranz, Gerardo Martinez, Conchi García, Josetxo Monserrat, Jorge Cabrejas, Sonia Giménez, Tere Pardo, Juanan Díaz, Irene Alepuz, Alicia Abad, Carlos Barjau, Dani Calabuig, Sandra Roger, David García, David Martín-Sacristán, Edu Garro, Manuel Fuentes, Jefferson Ribadeneira, former members of the group David Gozálvez, Jaime López, Jordi Calabuig and may others.

Last, but not least, I would like to thank my beloved parents, María Teresa and Segundo, my sister Cusi and my brother Segundo for all their unconditional love, support and encouragement through all the years. My most sincere gratitude goes to Soraya, for all her love and support during the course of this dissertation. Her endless patient during my multiple research stays and encouragement along all these years have been vital for the completion of this thesis. Thank you.

David E. Vargas Paredero

May 2016 


\section{Contents}

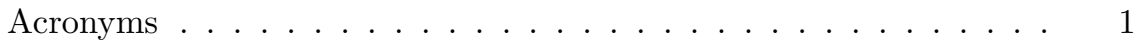

1 Introduction $\mathbf{5}$

1.1 State-of-the-Art of MIMO Signal Processing in DTT Systems . 11

1.2 Motivation and Problem Statement . . . . . . . . . . . 15

1.3 Objectives and Thesis Scope . . . . . . . . . . . . . . 17

1.4 Research Approach and Methodology . . . . . . . . . . . . 17

1.5 Thesis Outline . . . . . . . . . . . . . . . . . . 19

1.6 Research Contributions . . . . . . . . . . . . . . 21

1.7 List of Publications . . . . . . . . . . . . . . . 24

2 Preliminaries $\quad 27$

2.1 System Model Overview . . . . . . . . . . . . . . . . . . 28

2.2 MIMO Channel: Signal model, Gains and Capacity . . . . . . . 30

2.2.1 AWGN channel . . . . . . . . . . . . . . . . 30

2.2 .2 Fading . . . . . . . . . . . . . . . . . . 30

2.2.3 Multiple Antenna Channel . . . . . . . . . . . . 31

2.2.4 MIMO Channel Capacity ............ 34

2.3 A Fundamental Trade-Off and Transmission Approaches . . . . 37

2.3.1 Multiplexing-Diversity Trade-Off . . . . . . . . . . . . 37

2.3.2 Space-Frequency Block codes ........... . 38

2.3.3 Spatial Multiplexing Techniques . . . . . . . . . . . . 40

2.4 MIMO-BICM: Components and Mutual Information . . . . . . 44

2.4.1 Forward Error Correction . . . . . . . . . . . . . . . 44

2.4 Bit-Interleaver . . . . . . . . . . . . . 45

2.4.3 Mapping from Bits to Constellation Symbols . . . . . . 46

2.4.4 MIMO Demodulation and Iterative Decoding . . . . . . 48

2.4.5 Mutual Information of MIMO-BICM systems . . . . . 53

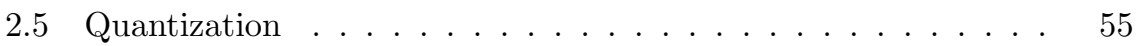


3 MIMO Precoding for Terrestrial Broadcast Systems $\quad 59$

3.1 Introduction and Scope . . . . . . . . . . . . . . . 60

3.2 Precoding Based on Rotation Matrices . . . . . . . . . . . . . 62

3.2 .1 BICM Capacity Analysis . . . . . . . . . . . . . . . 64

3.2 .2 System Performance . . . . . . . . . . . . . . . . 75

3.2.3 MIMO Precoding in DVB-NGH and ATSC $3.0 \ldots . .87$

3.3 Precoding Based on Maximum Ergodic Capacity . . . . . . . . 92

3.3.1 Design of MIMO-Channel-Precoders . . . . . . . . . 97

3.3.2 Capacity Analysis . . . . . . . . . . . . . . . . . . . . 99

3.3.3 System Performance . . . . . . . . . . . . . . . . . 104

3.4 Summary and Conclusions . . . . . . . . . . . . . . . . 113

4 Receiver Memory and Performance Trade-Off of Soft-Quantized Information $\quad \mathbf{1 1 5}$

4.1 Quantized receiver Architectures . . . . . . . . . . . . 116

4.2 Quantization Algorithms . . . . . . . . . . . . . . . 120

4.2.1 Quantization of I\&Q Signal Components . . . . . . . . 120

4.2.2 Quantization of Log-likelihood Ratios . . . . . . . . . 121

4.3 Performance Evaluation of Quantized Architectures . . . . . . . 126

4.4 Memory vs. Performance Trade-Off . . . . . . . . . . . . . . . 131

4.5 Summary and Conclusions . . . . . . . . . . . . . . 138

5 Conclusions and Outlook $\quad 141$

5.1 Conclusions . . . . . . . . . . . . . . . . . . . . 143

5.2 Outlook . . . . . . . . . . . . . . . . . . . 148

A MIMO Channels in Terrestrial Broadcasting: Models and Capacity 153

A.1 Channel Models . . . . . . . . . . . . . . . . . . . . . . . . 154

A.1.1 Basic Models . . . . . . . . . . . . . . . . . 154

A.1.2 Modified Guilford Rooftop Model . . . . . . . . . . . 156

A.1.3 Next Generation Handheld Models . . . . . . . . . . 158

A.1.4 Extension to Four Transmit Antennas . . . . . . . . . . 161

A.2 Ergodic Capacity with Asymptotic SNR . . . . . . . . . 162

A.3 Performance Evaluation . . . . . . . . . . . . . . . 163

A.3.1 Performance evaluation in basic channels . . . . . . . 163

A.3.2 Performance evaluation in Terrestrial broadcast channels 169

B DVB-NGH and ATSC 3.0 MIMO Physical Layers 177

B.1 Generic Transmit MIMO DVB-NGH \& ATSC 3.0 Physical layer 178

B.2 Overview of DVB-NGH and ATSC 3.0 Transmitting blocks . . 178

$\begin{array}{ll}\text { References } & 185\end{array}$ 


\section{Acronyms}

1D-NUC One-dimensional Non-Uniform Constellation

2D-NUC Two-dimensional Non-Uniform Constellation

3GPP Third Generation Partnership Project

ADC Analog-to-Digital Converters

ATSC Advanced Television Systems Committee

ATSC-M/H ATSC - Mobile/Handheld

ATSC 3.0 ATSC - Terrestrial Third Generation

AWGN Additive White Gaussian Noise

BCH Bose-Chaudhuri-Hocquenghem

BER Bit Error Rate

bpc bits per channel use

BICM Bit Interleaved Coded Modulation

cdf Cumulative Density Function

CM Coded Modulation

CMMB China Mobile Multimedia Broadcasting

CNR Carrier to Noise Ratio

CRSI Channel Receive State Information

CSI Channel State Information

CTSI Channel Transmit State Information

DMT Diversity-Multiplexing Tradeoff

DTMB Digital Terrestrial Multimedia Broadcast

DTT Digital Terrestrial TV

DVB Digital Video Broadcasting

DVB-C2 DVB - Cable 2nd Generation

DVB-H DVB - Handheld 
DVB-NGH DVB - Next Generation Handheld

DVB-SH DVB - Satellite services to Handheld devices

DVB-T DVB - Terrestrial

DVB-T2 DVB - Terrestrial 2nd Generation

E-MBMS Enhanced Multimedia Broadcast and Multicast Service

eSFN enhanced Single Frequency Network

eSM-PH Enhanced Spatial Multiplexing with Phase Hopping

FEC Forward Error Correction

FER Frame Error Rate

FOBTV Future of Broadcast Television

HDTV High Definition TV

HEVC High Efficiency Video Coding

IB Information Bottleneck

ISDB-T Integrated Services Digital Broadcasting - Terrestrial

ISDB-T2 ISDB - Terrestrial 2nd Generation

ITU International Telecommunication Union

LDPC Low Density Parity Check

LLR Log-Likelihood Ratio

LoS Line Of Sight

LTE Long Term Evolution

MAP Maximum a Posteriori

MFN Multi Frequency Network

MGM Modified Guilford Model

MIMO Multiple-Input Multiple-Output

MIMO-BICM MIMO - Bit Interleaved Coded Modulation

MIMO-BICM-ID MIMO-BICM with Iterative Decoding 


$\begin{array}{ll}\text { MISO } & \text { Multiple-Input Single-Output } \\ \text { MMSE } & \text { Minimum Mean Square Error } \\ \text { MSB } & \text { Most Significant Bit } \\ \text { MSE } & \text { Mean Squared Error } \\ \text { NLoS } & \text { Non Line Of Sight } \\ \text { OFDM } & \text { Orthogonal Frequency Division Multiplexing } \\ \text { PAM } & \text { Pulse Amplitude Modulation } \\ \text { pdf } & \text { Probability Density Function } \\ \text { PI } & \text { Power Imbalance } \\ \text { QoS } & \text { Quality of Service } \\ \text { RF } & \text { Radio Frequency } \\ \text { SDTV } & \text { Standard Definition TV } \\ \text { SFBC } & \text { Space Frequency Block Coding } \\ \text { SFN } & \text { Single Frequency Network } \\ \text { SHV } & \text { Super Hi-Vision } \\ \text { SIMO } & \text { Single-Input Multiple-Output } \\ \text { SISO } & \text { Single-Input Single-Output } \\ \text { SM } & \text { Spatial Multiplexing } \\ \text { SNR } & \text { Signal to Noise Ratio } \\ \text { STBC } & \text { Space Time Block Code } \\ \text { TDCFS } & \text { Transmit Diversity Code Filter Sets } \\ \text { TDI } & \text { Time de-Interleaving } \\ \text { T-DMB } & \text { Terrestrial - Digital Multimedia Broadcasting } \\ \text { TI } & \text { Time Interleaving } \\ \text { TV } & \text { Television } \\ \text { UHDTV } & \text { Ultra-High Definition TV } \\ & \end{array}$


UHF

WiMAX

WRC

XPD
Ultra High Frequency

Worldwide Interoperability for Microwave Access

World Radiocommunication Conference

Cross Polarization Discrimination 


\section{Chapter 1}

\section{Introduction}

TERRESTRIAL broadcasting systems are facing a new era in which the spec1 trum efficiency is forced to be significantly enhanced due to increasing demands of the scarce frequency resource in the Ultra High Frequency (UHF) band. Since the 1950s, Television (TV) was the most widespread and popular telecommunication system. The introduction of TV produced social changes, where the entire family and friends stopped daily activities to gather around this new technology device to watch the news, historic events, or entertainment TV. The switch from analogue to digital processing in the 1990s brought various benefits, such better use of the frequency spectrum, noise-free reception, High Definition TV (HDTV), flexible network parameter configurations (e.g., trade-off service area, quality reception, transmission power, data capacity and spectrum) and the introduction of multimedia or interactivity amongst others.

The utilization of broadcasting systems for the delivery of Digital Terrestrial TV (DTT) has grown very strongly during the last decade, and DTT networks are already in place in many countries all over the world. Several first generation broadcasting technologies are available today for the provision of DTT services: Advanced Television Systems Committee (ATSC) in North America, Integrated Services Digital Broadcasting - Terrestrial (ISDB-T) in Japan, Terrestrial - Digital Multimedia Broadcasting (T-DMB) in Korea, Digital Terrestrial Multimedia Broadcast (DTMB) in China, and the Digital Video Broadcasting (DVB) forum in Europe developed DVB - Terrestrial (DVB-T). Also, already deployed a second generation DTT system, DVB - Terrestrial 2nd Generation (DVB-T2) standard [1], which provides a $50 \%$ increase of spectral efficiency over its predecessor [2], and motivated by technological advances in communications and the appearance of new digital content such as HDTV. 


\section{Pressure on DTT spectrum}

The better utilization of the DTT spectrum has lead to the so-called digital dividend, defined as the amount of spectrum made available by the transition of terrestrial TV broadcasting from analogue to digital [3]. Part of released spectrum can be used by broadcasting services to accommodate more programs or with higher quality, or on the other hand it can be assigned to other services such mobile broadband. With the strong penetration of mobile terminals, such smart-phones and tablet computers, there is an exploding demand for mobile data traffic, which is estimated to increase nearly eightfold between 2015 and 2020, and video content will reach a significant percentage of this traffic [4].

It is obvious that mobile broadband networks need more resources to fulfil the current mobile data-tsunami in the form of new infrastructure or new spectrum bands. This served as motivation to the International Telecommunication Union (ITU) to allocate to mobile broadband the upper part of the $800 \mathrm{MHz}$ UHF band (i.e., from 790 to $862 \mathrm{MHz}$ ) in ITU region $1^{1}$, and the $700 \mathrm{MHz}$ band $(698-806 \mathrm{MHz})$ in ITU regions 2 and 3 . At the World Radiocommunication Conference (WRC) 2012 (WRC-12), the $700 \mathrm{MHz}$ band (694-790 MHz) in Region 1 was already assigned to broadcasting and mobile broadband on a co-primary basis. Recently, in the WRC-15 it has been decided to release the $700 \mathrm{MHz}$ band (also known as the second digital dividend) in ITU region 1, but it has also been decided that there would be no change to the allocation in the 470-694 MHz band either now, or at WRC19. However, there will be a review of the spectrum use in the entire UHF band (470-960 MHz) at the WRC in 2023.

Equally important in the pressure on DTT spectrum is the higher data rates required for the delivery of HDTV, new content such as Ultra-High Definition TV (UHDTV), and the pressure for all Standard Definition TV (SDTV) services to be converted to HDTV.

It is obvious that in this time of competitiveness, DTT networks have to significantly increase their spectrum efficiency to cope with the limited spectrum use, appearance of new content, and the ubiquity of the content (e.g., fixed, portable, mobile). To cope with these demands currently various international technical forums are envisaging new DTT technologies: ATSC - Terrestrial Third Generation (ATSC 3.0) in North America and ISDB - Terrestrial 2nd Generation (ISDB-T2) in Japan. At the same time, motivated by the high fragmentation of terrestrial broadcasting systems around the world, and the necessity to benefit from economies of scale, in November 2011 the Future of

\footnotetext{
${ }^{1}$ For the purpose of global radio spectrum management, the world is divided in three ITU regions. The first is formed by Europe, Africa, the Middle East west of the Persian Gulf including Iraq, the former Soviet Union and Mongolia. The second includes the Americas, Greenland and some of the eastern Pacific Islands. The third covers most of non-formerSoviet-Union Asia, east of and including Iran, and most of Oceania.
} 
Broadcast Television (FOBTV) initiative was launched. FOBTV aims to create next generation single world-wide terrestrial broadcast system [5].

\section{DTT Systems for the Delivery of Mobile Broadcast}

The transmission of mobile video is one of the most popular multimedia content currently conveyed through the networks, and since it requires higher data-rate that other multimedia contents (e.g., such audio or text), it absorbs notable part of the network's bandwidth. Estimations allocate around threefourths of the world's mobile data traffic will be video by 2020 [4]. DTT specifications for the provision of mobile services in handheld devices have been deployed by the main broadcasting organizations such as: ATSC - Mobile/Handheld (ATSC-M/H) [6], ISDB-T one-segment [7], or China Mobile Multimedia Broadcasting (CMMB) [8]. In Europe, the DVB forum specified DVB - Next Generation Handheld (DVB-NGH) [9] for the provision of mobile services to handheld devices outperforming in coverage and capacity previous mobile broadcasting standards such as DVB - Handheld (DVB-H) and DVB Satellite services to Handheld devices (DVB-SH).

\section{Technology Advancements for DTT Systems}

Higher spectral efficiencies are enabled by the utilization of new technological advancements. More sophisticated modulation and coding schemes can increase the system capacity or equivalently reduce the required Signal to Noise Ratio (SNR) for a service to be decodable. The use of advanced channel coding like turbo or Low Density Parity Check (LDPC) codes [10, 11] achieve a performance that is close to the theoretical limits in Additive White Gaussian Noise (AWGN) channel and reduces the gap with capacity in mobile channels. For instance, the switch from convolutional to LDPC codes brought $50 \%$ capacity increase from DVB-T to DVB-T2 [1]. Also, the progress in video compression schemes allows for significant bit rate reductions as for the High Efficiency Video Coding (HEVC) which can reduce by $50 \%$ the video bit rate compared with the previous H.264/AVC codec version [12].

However, the spectral efficiencies reached even with combining state of the art DTT system with performance close to capacity (e.g., DVB-T2) and latest video compression schemes (e.g., HEVC) may not to suffice to face present and future DTT spectrum demands. A significant increase in DTT spectral efficiency can be achieved is by multi-antenna technology, i.e., Multiple-Input Multiple-Output (MIMO). MIMO allows overcoming the capacity limit of single antenna wireless communications in a given channel bandwidth without any increase in the total transmission power [13]. The spectral efficiencies achieved with MIMO and new video compression schemes have the potential to cope with present demands of DTT spectral efficiency. 
MIMO can be a key technology for future broadcasting systems, which increases the system capacity and the signal resilience without any additional bandwidth or increased transmission power. However these gains come at expense of additional hardware and more sophisticated signal processing at both sides of the transmission link. Because of its potential, MIMO has become part of wireless standards such as: IEEE 802.11n for wireless local area networks, Worldwide Interoperability for Microwave Access (WiMAX) for broadband wireless access area systems, and Third Generation Partnership Project (3GPP)s Long Term Evolution (LTE) for cellular networks amongst others.

\section{Multiple-Input Multiple Output (MIMO) for DTT Systems}

In DTT broadcasting world, DVB-NGH and ATSC 3.0 incorporate multiantenna technology exploiting the benefits of the MIMO channel [14, 15]. Similarly, ISDB-T2 [16], FOBTV, and DVB with a potential extension to DVB-T2 are also considering MIMO. MIMO has proved the potential of $80 \%$ capacity increase over Single-Input Single-Output (SISO) in mobile scenarios [14], and higher capacity gains are expected in rooftop reception due to higher signal strength levels where figures of $25 \mathrm{~dB}$ of Carrier to Noise Ratio (CNR) are practical [17]. An important difference between DTT and cellular systems is the lack of a return channel from the receivers to the transmitters, which prevents link adaptation and/or beamforming techniques in DTT systems.

Multi antenna techniques provide three important gains, i.e. array gain, diversity gain, and multiplexing gain [18], and illustrated in Fig. 1.1. Array gain increases the received CNR with coherent combination at the receive side (signal co-phasing and weighting for constructive addition). Here, information about channel state information is required, and it is commonly obtained by tracking the channel variations with the transmission of pilot signals. Diversity gain improves the reliability of the transmission by sending the same information through independently faded spatial branches to reduce the probability that all channels are in a deep fade. In addition to array and diversity gains, the MIMO channel can increase the system capacity by transmitting independent data streams across the transmit antennas through spatial multiplexing. MIMO techniques can be used to improve the robustness of the transmitted signal by exploiting the spatial diversity of the MIMO channel, but also to achieve increased data rates through spatial multiplexing especially at high CNR values [13], e.g., outdoor medium/high signal use cases such as vehicular reception or fixed rooftop reception.

The main drawback for implementing MIMO is that existing DTT network infrastructure needs to be upgraded at both transmitter (e.g., additional transmit antennas, Radio Frequency (RF) feedings, power combiners and amplifiers, 


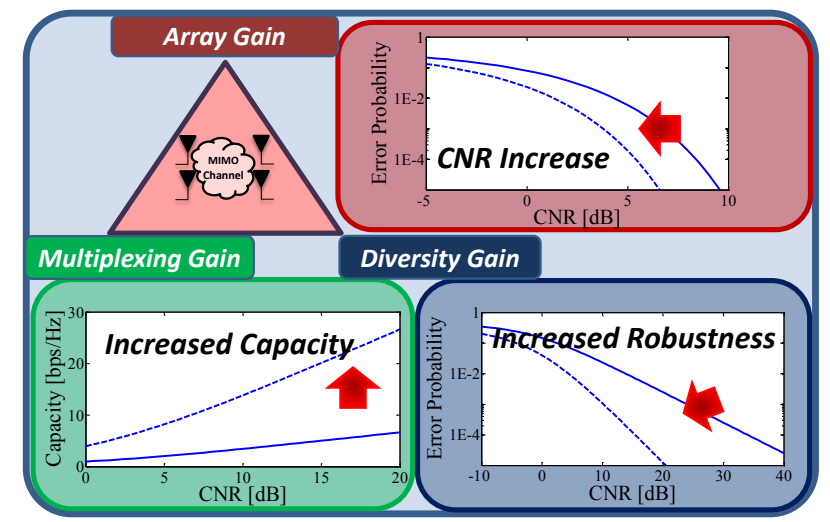

Figure 1.1: MIMO gains. Array gain produces an average increase in the received CNR, diversity gain increases the resilience against fading, and multiplexing gain increases the capacity of the system.

etc.) and receiver sides (more sophisticated signal processing with more antennas and RF front ends for demodulation). However, the deployment impact can be reduced at the consumer receivers by timely combining MIMO implementation and HEVC codec upgrade in the same wave of digital switch-overs. Additionally, to demodulate an MIMO signal, it is necessary to employ orthogonal pilot patterns between antennas, so that each receive antenna can estimate the channel response from each transmit antenna. Compared with single antenna transmissions, the required number of transmitted pilots must be doubled (with two transmit antennas) for the same resolution of channel estimation, hence reducing the overall spectral efficiency.

The operation in the UHF band of DTT systems have motivated the adoption of cross-polar ${ }^{2}$ antenna configuration (i.e., antennas with vertical and horizontal polarizations). Compared with the co-polar counterpart (antennas with the same polarization), cross-polar antennas provide higher multiplexing gains in Line Of Sight (LoS) conditions due to orthogonal nature of the cross-polar channel [17]. Moreover, in the UHF frequency range, the antenna separation required in the co-polar case to provide sufficiently independent fading signal may exceed typical handheld devices sizes.

\footnotetext{
${ }^{2}$ In the literature the antenna configuration of a MIMO $2 \times 2$ system with antennas with orthogonal polarizations is referred as: dual-polar antennas, polarized antennas, orthogonally polarized antennas, and cross-polar antennas. In this thesis the term cross-polar antennas will be used.
} 


\section{MIMO Broadcast Field Trials}

The potentials of MIMO for DTT with cross-polar transmission were recognized in early work of Monnier et al. in [19] in 1992. Latter in 2006, the public broadcaster BBC (UK) performed initial tests of MIMO broadcast with crosspolar antennas in laboratory and small scale field measurements [20], which lead to a high-power field trial in Guildford (UK) [21]. This field test showed the potential of doubling the system bit-rate obtained with DVB-T in the same $8 \mathrm{MHz}$ channel with similar coverage area. In addition, this field test allowed the creation of a channel model representative of fixed reception at rooftop level with cross-polar antennas [22].

During the standardization process of DVB-NGH, a channel sounding campaign took place in July 2010 in Helsinki, Finland, focussing on cross-polar UHF transmission and reception in order to compare candidate schemes in simulation with a realistic measurement-based channel model [17]. The trials included a number of practical designs for antennas suitable for a handheld terminal. From the measurements, the following channel models were developed: an outdoor mobile model, an outdoor portable model and an indoor portable model. For the mobile case, a user velocity of $350 \mathrm{~km} / \mathrm{h}$ or $60 \mathrm{~km} / \mathrm{h}$ are considered, whereas for the portable case, user velocities of $3 \mathrm{~km} / \mathrm{h}$ or $0 \mathrm{~km} / \mathrm{h}$ are considered.

The Japanese public broadcaster (NHK) has performed various field trials and research experiments of cross-polar MIMO under the development of the Super Hi-Vision (SHV) video system for fixed and mobile reception. In 2012, a transmission was achieved with cross-polar MIMO with LDPC codes and a constellation order of 4096QAM [23]. Such a high spectral efficiency is required to support the very high data rates of the $\mathrm{SHV}$ video system. The performance in mobile environments using Space Frequency Block Coding (SFBC) was evaluated by simulations and field experiments [24]. Results in [16, 25] present field trials with two transmitter sites in a in Single Frequency Network (SFN) configuration, forming a $4 \times 2 \mathrm{MIMO}$ system. In this trial the use of Space Time Block Code (STBC) provides enhanced performance over conventional SFN transmitting the same signal at each site.

Although MIMO in DTT systems has already been included in DVB-NGH and ATSC 3.0, and multiple field trials have been performed, there are currently no commercial deployments of DTT systems using MIMO with multiple antennas at the transmitters and the receivers.

DVB-T2 specification did not include cross-polar MIMO because the commercial requirements mandated the reuse of existing domestic receiving antenna installations and of existing transmitter infrastructures. For this reason DVB-T2 adopted a Multiple-Input Single-Output (MISO) scheme based on a site distributed configuration with SFBC reusing the transmitter infrastruc- 
ture. The SFBC scheme adopted in DVB-T2 is based on Alamouti coding [26] defined to exploit only diversity gain. The Alamouti code is designed for achieving full diversity with reduced (linear) complexity at the receiver side with two transmit antennas. By using the Alamouti code in a distributed manner it is possible to improve the performance in SFN transmission. The arrival of similar-strength signals from different transmitters in LoS scenarios can cause deep notches in the frequency response of the channel degrading the performance in an important manner. The Alamouti code requires orthogonal pilot patterns between antennas which imposes doubling the number of transmitted pilots to achieve the same resolution of channel estimation as with single antenna transmissions. Despite the potential improvements shown in theory by the distributed MISO scheme in SFN scenarions, results from simulation in [27] and field measurements carried out in Germany in portable and mobile scenarios do not recommend the use of MISO [28] based on Alamouti coding.

\subsection{State-of-the-Art of MIMO Signal Process- ing in DTT Systems}

\section{Transmit MIMO Signal Processing}

DVB-NGH is the first broadcast system to employ MIMO (i.e., with spatial multiplexing gains) as key technology exploiting the benefits of the MIMO channel (cf. Fig. 1.1). In DVB-NGH two types of MIMO schemes are specified which are detailed next.

The first type of techniques is known in DVB-NGH as MIMO rate 1 codes, which exploit the spatial diversity of the MIMO channel without the need of multiple antennas at the receiver side. They can be applied across the transmitter sites of SFNs to reuse the existing network infrastructure (i.e. DVB-T and DVB-T2), as well as to an individual multiple-antenna transmitter site. These codes are specified as part of the base (sheer-terrestrial) profile and include the Alamouti code, already featured in DVB-T2, together with a scheme known as enhanced Single Frequency Network (eSFN). The main idea of eSFN is to apply a linear pre-distortion function to each antenna in such a way that it does not impact the channel estimation. This technique increases the frequency diversity of the channel without the need of specific pilot patterns or signal processing to demodulate the signal. eSFN is also well suited for its utilization in a distributed manner, as the randomization performed in each transmitter can avoid the negative effects cause by LoS components in this kind of networks [29].

The second type of techniques is known in DVB-NGH as MIMO rate 2 codes, which exploit the diversity and multiplexing capabilities of the MIMO 
channel. In this category DVB-NGH has adopted a novel scheme known as Enhanced Spatial Multiplexing with Phase Hopping (eSM-PH). The most simple way of increasing the multiplexing rate of information consists on dividing the information symbols between the transmit antennas, this is referred to as MIMO Spatial Multiplexing (SM). However, the presence of correlation in the MIMO channel due to LoS channel condition, which frequency happens in terrestrial broadcast transmissions, is especially detrimental for MIMO SM. eSM-PH was designed to overcome this situation retaining the multiplexing capabilities of MIMO SM, and at the same time, increasing the robustness against spatial correlation. With eSM-PH the information symbols are weighted and combined before their transmission across the antennas according to a specified rotation angle. This rotation angle has been tuned for every combination of constellation order and deliberated transmit power imbalance. To ease dual polar operation, MIMO rate 2 codes can be transmitted with deliberated power imbalance between the transmit antennas with values of $0 \mathrm{~dB}, 3 \mathrm{~dB}$ and $6 \mathrm{~dB}$. In addition, eSM-PH includes a periodical phase hopping term to the second antenna in order to randomize the code structure and avoid the negative effect of certain channel realizations. It is important to note that the implementation of eSM-PH scheme requires additional investment at both sides of the transmission link. For this reason, these codes are specified as an optional profile to allow for a progressive deployment of MIMO networks depending on the market demands. In addition to demodulate an eSM-PH signal it is necessary to employ orthogonal pilot patterns (as for the Alamouti code) between antennas.

MIMO for DVB-NGH targets mobile terminals operating in the low-tomedium spectral efficiency range. High data rate services operating in the high spectral efficiency range for rooftop reception are addressed by the development of ATSC 3.0. ATSC 3.0 includes a wider range of spectral efficiencies than DVB-NGH and in addition it uses non-uniform constellations to provide additional shaping gains [15]. The MIMO precoding scheme adopted in ATSC 3.0 is composed of a rotation matrix (as in DVB-NGH), an In-phase and Quadrature (I\&Q) polarization interleaver and a phase hopping blocks [15]. Each of the three sub-blocks of the MIMO precoder can be optionally selected. The phase hopping block adopted in ATSC 3.0 is the same as in DVB-NGH. The I\&Q polarization interleaver is simply a switching interleaving operation, such that the output cells consist of the real (In-phase) component of one input symbol and the imaginary (Quadrature) component of the other input symbol. A more sophisticated inter-polarization spreading techniques is proposed in [30], to improve the performance under high correlated channels or reception with one antenna.

The use of MIMO in SFN networks has been studied by the hybrid terrestrialsatellite profile of DVB-NGH. The terrestrial and satellite transmitters can form a SFN network where either the terrestrial site or the satellite can have 
multiple transmit antennas. During the DVB-NGH standardization multiple rate 1 and rate 2 SFBCs were proposed to optimize the performance in the distributed scenario [31, 32]. Other codes have been proposed for DTT systems to optimize the performance in SFN scenarios as in [33] with high decoding complexity and similarly in [34] with a code design to significantly reduce the receiving computational complexity. A good survey of MIMO SFBCs in DTT scenarios can be found in [35].

\section{Feature Comparison with Long Term Evolution (LTE) Multi-Antenna Techniques}

LTE/LTE-Advanced is a wireless standard for cellular networks with multiantenna technology [36]. The main physical difference with broadcasting systems is the feedback channel from the receiver to the transmitter that allows for link adaptation techniques in LTE/LTE-Advanced. Link adaptation techniques improve the communication from the transmitter to the receiver by dynamically adapting key transmission parameters (e.g. modulation, coding rate, antenna weight vector for transmit beamforming) to the channel variations occurred in time, frequency and/or space.

Note that LTE/LTE-Advanced does not include MIMO for its multicast broadcast extension known as Enhanced Multimedia Broadcast and Multicast Service (E-MBMS). However, the MIMO schemes adopted for DVB-NGH and ATSC 3.0 share similar technologies with LTE/LTE-Advanced open loop single user downlink schemes as the way to exploit the benefits of the MIMO channel. Regarding the number of transmitting elements, MIMO DTT systems define a maximum of two transmit antennas, while for LTE the maximum number is four, and eight for LTE-Advanced. Increasing the number of transmit antennas generally improves the system performance at expense of higher system complexity.

\section{Receive MIMO Signal Processing}

Bit Interleaved Coded Modulation (BICM) [37, 38] is a pragmatic approach to coded Coded Modulation (CM) [39] where a bit-wise interleaver permits the independent design of modulation and coding. Design flexibility and robustness against fading have motivated rapid extensions to MIMO systems and its combination with Orthogonal Frequency Division Multiplexing (OFDM) modulation. MIMO - Bit Interleaved Coded Modulation (MIMO-BICM) is a key technology for modern wireless communications systems, such the specified DVB-NGH and ATSC 3.0. Although BICM with single antenna systems can provide performance close to capacity [40], there is still a substantial gap between MIMO-BICM and the multi-antenna system capacity. MIMO-BICM 
with Iterative Decoding (MIMO-BICM-ID), where MIMO demodulator and the channel decoder exchange extrinsic information in an iterative fashion, has the potential to reduce the gap to the CM bound. However, the performance improvements of iterative decoding the MIMO-BICM system come at expense of higher computational complexity, making it less suited for mobile devices. To reduce the computational complexity, numerous suboptimal MIMO receivers have been proposed, e.g., sphere decoding implementation [41, 42] and Minimum Mean Square Error (MMSE) receivers [43].

The performance of MIMO-BICM-ID was evaluated during the standardization of the MIMO profile of DVB-NGH due to the introduction of a bitinterleaver, which exhibits low complexity, low latency, and fully parallel design that ease the implementation of iterative structures. The bit-interleaving is based on permutation sequences that have been optimized for the different combinations of constellation and code-rate and transmit power imbalance. Furthermore, these selected permutations optimize the gain achieved by MIMO-BICM-ID receivers. The evaluations of MIMO-BICM-ID showed an increasing iterative decoding gain at increasing code-rate [14, 44].

During the standardization of new communication systems the initial design accounts for perfect reception conditions, e.g., optimal demodulators, perfect Channel State Information (CSI), perfect noise power estimation, and infinite-precision number representations. For instance, that was the approach to design DVB-T2, DVB-NGH and ATSC 3.0. However, due to complexity constraints and finite-precision arithmetic, it is crucial for the overall system performance to carefully design receiver algorithms. In addition since on-chip memory accounts for a large fraction of the chip area, it is desirable to have small word length with reduced performance loss.

DTT systems such as DVB-T2, DVB-NGH and ATSC 3.0 rely on Time Interleaving (TI) techniques to overcome signal fluctuations and improve the system performance. Yet, TI imposes the highest in-chip memory requirements [45], which depend on the quantization resolution and algorithms employed at the receiver terminal. DVB-T2 defines the same TI memory in number of cells (which represent a modulated OFDM carrier) irrespectively of the modulation and coding configured [46]. Using a constant number of cells irrespective of the modulation and coding leads to an important reduction in the maximum data rate and maximum TI depth duration for low order constellations [46]. It is important to note that the final amount of memory in bits required for the time-deinterleaving block directly depends on the resolution of the quantization algorithms. Since low order constellations, such as QPSK, can tolerate higher quantization noise levels, DVB-NGH introduced the concept of adaptive cell quantization [47]. Adaptive cell quantization permits, for a given time-deinterleaving memory size in bits, increasing the number of cells of the TI block for low order constellations while maintaining the interleaving depth 
for higher order constellations. Numerical evaluations for different quantization resolutions for the I\&Q components showed that the number of cells of the TI block can be doubled for QPSK and 16QAM compared to 64QAM and 256QAM for similar performance degradation [47]. Similarly, ATSC 3.0 has also introduced adaptive cell quantization scheme in its specification.

\subsection{Motivation and Problem Statement}

MIMO in DTT networks has the potential to increase the spectral efficiency and improve network coverage to cope with the competition of limited spectrum use, the appearance of new high data rate services, and the ubiquity of the content. The MIMO benefits can be achieved without additional transmit power nor additional bandwidth, but normally come at the expense of a higher system complexity at the transmitter and receiver ends. However, the final gains due to the use of MIMO directly depend on physical characteristics of the propagation environment such as spatial correlation, antenna orientation, and/or power imbalances experienced at the transmit aerials. Additionally, due to complexity constraints and finite-precision arithmetic at the receivers, it is crucial for the overall system performance to carefully design specific signal processing algorithms.

DVB-NGH and ATSC 3.0, the DTT systems with the most advanced terrestrial broadcast transmission technologies, have adopted MIMO as key technology to overcome the Shannon limit of single antenna communications. DVBNGH targeting mobile and portable reception, and ATSC 3.0 targeting mobile, portable and fixed reception, implement similar MIMO precoding solutions based on rotation matrices for $2 \times 2 \mathrm{MIMO}$. Hence, the performance analysis of MIMO precoding techniques for DTT systems in realistic cross-polar MIMO channels in the UHF band targeting mobile, portable, and fixed rooftop reception is an important research topic. Additionally, a characterization of the precoder performance against typical characteristics of the MIMO cross-polar channel in the UHF band (such as antenna correlation and/or transmit power imbalance) and/or transmission parameters (such as the transmit constellation order and/or the channel code-rate) is of significant research interest.

Other interesting topic of research is precoding which can exploit statistical information of the MIMO channel. Previous works in the literature such as in references $[48,49,50,51]$ have studied MIMO precoding schemes when transmitters have only available statistical information of the channel due weak feedback information of high mobility channels, for instance. However, it has not been applied to the MIMO terrestrial broadcast set-up. Additionally, the design of precoders for $4 \times 2$ MIMO is an interesting topic due to initial consid- 
erations of $4 \times 2$ MIMO set-ups in the MIMO study mission of the DVB fora for a potential extension of DVB-T2 specification.

So far, receiver hardware design has taken into account finite-precision number representations only in a straightforward manner via simulations, completely neglecting non-uniform quantizers adjusted to the signal properties. In addition since on-chip memory accounts for a large fraction of the chip area, it is desirable to have small word length with reduced performance loss. DTT systems, such as DVB-NGH, rely on TI techniques to overcome signal fluctuations and improve the system performance by time-diversity gain. Yet, long TI imposes the highest in-chip memory requirements at the receiver, which depends on the quantization resolution and specific algorithms. With the introduction of adaptive cell quantization [47] during the standardization of DVB-NGH (and recently in ATSC 3.0), uniform quantization of I\&Q signal component has been considered and evaluated. However, neither quantization of Log-Likelihood Ratio (LLR)s nor non-uniform quantizers adapted to the signal statistics have been considered during the development of DVB-NGH or ATSC 3.0. On the other hand, [52] proposes non-uniform quantization of LLRs to reduce the time de-interleaving memory for a DVB - Cable 2nd Generation (DVB-C2) system. However, quantization of I\&Q signal component was not considered in this work and the algorithms were applied to a SISO system. Hence, investigations on the topic of quantized MIMO-BICM receivers in DTT systems comparing I\&Q signal components and LLR quantization is an important research topic. 


\subsection{Objectives and Thesis Scope}

The main research topic of this dissertation is the design, characterization and evaluation of transmit and receive signal processing for DTT systems employing MIMO-BICM. In particular, the following specific objectives are defined:

\section{Transmit MIMO Signal Processing}

- To analyse the structure of the precoding based on rotation matrices for MIMO $2 \times 2$ systems as the one proposed in DVB-NGH and ATSC 3.0.

- To characterize the performance of the MIMO $2 \times 2$ precoding based on rotation matrices against important channel parameters in DTT systems in the UHF band, and different transmission parameters.

- To compare the results and conclusions obtained in the research of MIMO $2 \times 2$ precoding based on rotation matrices with the solutions adopted in DVB-NGH and ATSC 3.0.

- To develop new precoding schemes for DTT systems which exploit statistical information of the MIMO channel for $2 \times 2$ and $4 \times 2$ MIMO systems.

\section{Receive MIMO Signal Processing}

- To investigate the performance of uniform and non-uniform quantization of IEQ signal components and LLRs in MIMO-BICM receivers with iterative decoding.

- To investigate the impact on the in-chip memory and receiving architecture of quantization of IEQ signal components and quantization of LLRs.

- To study the performance and in-chip memory trade-off of quantized MIMO-BICM receiver with iterative decoding.

\subsection{Research Approach and Methodology}

This thesis includes design of communication algorithms (e.g., MIMO precoding techniques and practical iterative MIMO receivers based on quantized information) and system performance evaluation (e.g., evaluation of DVB and ATSC terrestrial standards). It is noted that despite the focus is primarily under the scope of DTT standards, the investigations will embrace a more general scope as design of communication techniques for future wireless broadcast communication systems using MIMO-BICM. 


\section{CHAPTER 1. INTRODUCTION}

The evaluation processes will be mostly driven by numerical simulations. In practice, the performance evaluation of a new communication system can be addressed by formula-based calculations, waveform-level simulation, or through hardware prototyping and measurements [53], and not mutually exclusive. In first stages of a system design simulation-based approach provides with a cost and flexible tool where different system/component configurations and tradeoff can be efficiently tested. Simulators can model with desired level of detail the information flow along the consequent blocks. However, the computational complexity of current algorithms, such MIMO demodulation or channel decoding, is significantly increasing and the simulation time to reach acceptable low error rate criteria can be extremely time demanding. On the other hand, formula-based techniques are mathematical expressions for different performance measures (Bit Error Rate (BER), Frame Error Rate (FER), etc.) dependent of the system parameters. They require very low computational complexity but are, in general, very complex to obtain or various assumptions have to be integrated in the model which in turns reduces the accuracy of the predictions. This thesis aims to design and evaluate DTT communication systems in various environments (e.g., fixed, portable, and mobile) with most of its components (e.g., channel encoders, modulators, interleavers, etc) and with practical channel models extracted from measurement campaigns; therefore, exact closed-form expression are extremely complex to obtain. Naturally, hardware implementations provide massive computational time gains in addition to accurate and realistic results. Still, it usually involves longer development time, lower flexibility and higher expenses in equipment. Due to these reasons, hardware implementations are not commonly used at the early stages of design cycle when high flexibility is required. In this thesis the design and evaluation methodology will be mainly based of a combination of system numerical simulations and derivation of mathematical expressions due to the requirement of flexibility on design and feasible mathematical tractability.

The performance evaluation of the different signal processing algorithms in this thesis will be carried in the context of the DTT systems. To this end a simulation platform supporting the MIMO profiles of the DVB-NGH and ATSC 3.0 physical layers is developed. This development includes the MIMO cross-polar channel models in the UHF band for mobile/portable reception (i.e., NGH channel models), for fixed rooftop reception (MGM channel mode), and other basic channels detailed in the Appendix A. These platforms have been calibrated during the standardization of DVB-NGH and ATSC 3.0 with the simulation platform of other entities showing good alignment.

The numerical evaluations will average enough channel realizations to provide statistically reliable results. To this end a number of minimum number of simulated frames and a minimum number or erroneous detected frames is defined. The error criteria for the evaluations is selected in each case as a 
trade-off between simulation accuracy and simulation time and common values used in this dissertation are BER $10^{-4}$ and $10^{-5}$. Specific simulation stopping conditions and error rate criteria will be provided in each section prior the evaluation results.

Transmit MIMO signal processing: In this part of the thesis, the MIMO channel precoders based on rotation matrices and the proposed channel precoder will be evaluated with information theoretic measures, and with system BER. Information theoretic measures allow us to study the performance of the precoders in ideal transmit and receive conditions, providing upper limits for the maximum achievable rate of a practical implementation. For the performance characterization of the MIMO precoding based on rotation matrices, the mutual information of the BICM and CM systems will be used. For the performance characterization of MIMO-Channel-precoder based on statistical information the channel capacity will be used. On the other hand system BER evaluations provide us with a performance closer to a practical implementation with specific of transmit and receiving algorithms. In this case both type of precoders will use the BER criteria to characterize the minimum CNR at a given error-rate criteria. For the evaluations of the $4 \times 2$ MIMO schemes, the DVB-NGH physical layer simulation platform will be extended to support four antennas and information streams.

Receive MIMO signal processing: Specifically to this work, the simulation platform has been extended to include MIMO-BICM iterative decoding and the uniform/non-uniform quantizers of I\&Q signal components and LLRs. The performance of the unquantized MIMO-BICM receiver with iterative decoding was also calibrated during the standardization process of DVB-NGH since the bit-interleaving of the MIMO profile was optimized to improve the iterative decoding gain. In this part of the thesis the goal is to evaluate the performance of practical receivers, hence, the comparisons will be carried using system BER.

\subsection{Thesis Outline}

This thesis is divided in five chapters and two appendices which are outlined next.

Chapter 2 presents fundamental concepts on wireless communications needed for the rest of the thesis. First it presents the fundamentals of MIMO channels and the fundamental trade-off in MIMO communication which allows to introduce two important transmission approaches the so-called SFBC and spatial multiplexing schemes. Then it provides a description of the different 
components and their interactions of MIMO-BICM systems and presents its information theoretic limits. Finally, this chapter concludes introducing the concept of iterative demapping and quantization of the received information which permits signal processing with finite resolution. The theoretical and practical description of the communication components used in this thesis will allow to present more in-depth the contributions for this thesis work.

Chapter 3 first studies the performance and structure of MIMO precoders based on rotation matrices for $2 \times 2 \mathrm{MIMO}$ and focus on the case of cross-polar antennas, which is the preferred configuration in DTT systems in the UHF band. Interesting insights are obtained by analysis and evaluation with the information-theoretic limits of BICM systems and bit-error-rate simulations including channel coding. Then, a channel-precoder is proposed that exploits statistical information of the MIMO channel. The performance of the channelprecoder is evaluated in a wide set of channel scenarios and mismatched channel conditions, a typical situation in the broadcast set-up. Capacity and system performance simulations study the benefits and drawbacks of the proposed MIMO-channel-precoder.

Chapter 4 investigates memory and performance trade-offs of soft-quantized information in MIMO-BICM receivers. Two types of quantized receivers are investigated: quantization of I\&Q samples and quantization of LLRs. The implications on the in-chip memory and the possibility of implementing MIMOBICM with iterative decoding are presented and discussed. Additionally, the performance degradations of uniform quantization and non-uniform quantization algorithms is evaluated showing significant potential benefits for nonuniform quantization adapted to the signal statistics. The results obtained in this chapter highlight the important trade-off between in-chip memory and performance for quantized receiver architectures.

Chapter 5 summarizes the main contributions of this thesis and suggests further topics of research beyond the results of this work.

Appendix A presents the potential gains of multi-antenna techniques in terms of ergodic and outage capacities for representative channel models in terrestrial broadcast TV systems. It first starts with the evaluation with some basic channels widely used within literature and then proceed with the analysis with channel models extracted from channel measurement campaigns in the UHF band. The potential gains over the single antenna transmitter and receivers, which is the most representative case of the current DTT deployment worldwide, are given. Here, the performance evaluation considers the case where the information of the channel state is available only at the receiver (CRSI) and when is also available at the transmitter (CTSI). 
Appendix B describes the MIMO physical layers adopted for DVB-NGH and ATSC 3.0 specifications. Both specifications share similar architecture for the implementation of MIMO.

\subsection{Research Contributions}

\section{Precoding for MIMO $2 \times 2$ based in Rotation Matrices}

- Performance characterization of the MIMO $2 \times 2$ precoding based on rotation matrices against: typical channel characteristics of the MIMO cross-polar channel in the UHF band, and transmit constellation order and channel code-rate. Results based on Monte-Carlo simulations of the information theoretical limits of MIMO-BICM systems and system biterror-rate with practical channel codes show the interesting result that in general the optimal rotation angle depends on the system code-rate. In particular, the rotation gain increases for increasing code-rate, i.e., at low code-rates it is optimal not to rotate, and at mid and high code-rates it is optimal to rotate. It has been found that the gain achieved with rotation, on the other hand, decreases with increasing constellation orders. The study of the effect of power imbalance between transmit antennas, which is an important scenario in the broadcast-set-up, shows that the rotation performance has the same behaviour as with equal power between transmit antennas. However, in this case the rotation gains are even higher, increasing for increasing power imbalance factor.

- Performance characterization of the stream-power-allocation matrix with constellations of different cardinality in each transmit antenna. The evaluation in the case of cross-polar antennas shows that allocation of more power to the constellation with higher cardinality can improve the performance from mid to high code-rates. This can be achieved by either intentional transmit power imbalance between antennas or stream-power allocation. The rotation matrix in this case allows for a flexible configuration of the desired power allocation to the asymmetric information streams.

- Analysis and comparison of the precoding solutions adopted in DVB-NGH and $A T S C$ 3.0. A comparison with the results and conclusions obtained in this thesis show that the adopted parameters for the precoding in DVB-NGH can be suboptimal for some transmission configurations due to the use of the same rotation angle at all transmitted code-rates. For ATSC 3.0 case, a per code-rate rotation angle optimization allows optimal rotation performance at each transmitted spectral efficiency. 


\section{MIMO Precoding based on Channel Statistical Information}

- A MIMO channel-precoder that exploits statistical information of the MIMO channel for the terrestrial broadcasting setting. This precoder has the potential to further increase the channel capacity when compared to equivalent unprecoded MIMO set-up. The performance of the proposed channel-precoder is evaluated for fixed and portable channels and various reception conditions. A mismatched analysis allows to evaluate the performance of the precoder when the channel statistics do not match the precoder, a typical situation in the broadcast set-up. Capacity results present performance enhancements in scenarios with strong LoS correlated antenna component, and resilience in mismatched condition for the considered $4 \times 2$ MIMO systems but no enhacement for $2 \times 2$ MIMO systems. BER simulation results with the DVB-NGH MIMO physical layer show that for low code rates, performance improvements can be achieved in the case of strong LoS correlation and resilience against mismatched condition with the channel statistics.

\section{Receiver Memory and Performance Trade-Off of Soft-Quantized In- formation}

- Performance comparison of uniform and non-uniform quantizers for IEQ signal components and LLRs. The numerical evaluations show that nonuniform quantizer design adapted to the signal statistics provide significant improvements in terms of system performance or alternatively inchip memory savings. This has been verified for both quantized architectures, quantized I\&Q components and quantized LLRs, but showing higher enhancement for the latter.

- Impact of the type of quantization in the receiving architecture. An analysis of the receiving architectures with quantized I\&Q signal components and quantized LLRs unveils than the latter is not tailored to perform MIMO-BICM with iterative decoding. This is due to the exchange of demapping and time de-interleaving processing blocks. A receiving architecture with quantized I\&Q signal components does not have such a restriction since all the necessary signals for demapping with prior information are quantized and stored into memory.

- Impact of the type of quantization in the in-chip receiving memory. Due to the highest in-chip memory requirements are imposed by the time deinterleaving memory in DTT systems, this processing block is taken as reference for memory size calculations. An further study of the in-chip memory requirements shows lower memory requirements for a receiving architecture with quantized LLRs than for a receiving architecture with 
quantized I\&Q signal components. From the results it can be concluded that for similar quantization performance degradation, LLR quantization requires around half the memory compared to the in-chip memory with I\&Q quantization (assuming non-uniform quantizers).

- In-chip memory vs. performance trade-off of MIMO-BICM receivers with quantized soft-information. Due to the interrelationships between type of quantization architecture, MIMO-BICM with iterative decoding and inchip memory, this thesis presents the in-chip memory vs. performance trade-off to characterize MIMO-BICM receivers with quantized information. Quantization type selection guidelines are provided depending on the complexity and in-chip memory restrictions imposed at the receiver. 


\subsection{List of Publications}

\section{International journals}

[J1] D. Gozálvez, D. Gómez-Barquero, D. Vargas, and N. Cardona, "Time Diversity in Mobile DVB-T2 Systems," IEEE Transactions on Broadcasting, vol. 57, no. 3, pp. 617-628, September 2011.

[J2] D. Vargas, D. Gozálvez, D. Gómez-Barquero and N. Cardona, "MIMO for DVB-NGH, The Next Genaration Mobile TV Broadcasting," IEEE Communications Magazine, vol. 51, no. 7, pp. 130-137, July 2013.

[J3] D. Gozálvez, D. Gómez-Barquero D. Vargas and N. Cardona, "Combined Time, Frequency and Space Diversity in DVB-NGH," IEEE Transactions on Broadcasting, vol.59, no. 4, pp. 674-684, December 2013.

[J4] D. Vargas , Y. J. D. Kim, J. Bajcsy, D. Gómez-Barquero, and N. Cardona, "A MIMO-Channel-Precoding Scheme for Next Generation Terrestrial Broadcast TV Systems," IEEE Transactions on Broadcasting, vol. 61, no. 3, pp. 445-456, September 2015.

[J5] M. Fuentes, D. Vargas, and D. Gómez-Barquero, "Low-Complexity Demapping Algorithm for Two-Dimensional Non-Uniform Constellations," IEEE Transactions on Broadcasting, 2016.

[J6] S. LoPresto, R. Citta, D. Vargas, and David Gómez-Barquero, "Transmit Diversity Code Filter Sets (TDCFS), a MISO Antenna Frequency Pre-Distortion Scheme for ATSC 3.0," IEEE Transactions on Broadcasting, vol. 62, no. 1, pp. 271-280, March 2016.

[J7] D. Gómez-Barquero, D. Vargas, M. Fuentes, P. Klenner, S. Moon, J.-Y. Choi, D. Schneider, and K. Murayama, "MIMO for ATSC 3.0," IEEE Transactions on Broadcasting, vol. 62, no. 1, pp. 298-305, March 2016.

\section{National journals}

[NJ1] D. Vargas, D. Gozálvez, D. Gómez-Barquero, and N. Cardona, "Multi Antenna Techniques for Digital Video Broadcasting (DVB) Systems," Waves, year 4, pp. 79-88, 2012.

\section{Book chapters}

[B1] D. Vargas, S. Moon, W.-S. Ko, and D. Gómez-Barquero. "Enhanced MIMO Spatial Multiplexing with Phase Hopping for DVB-NGH," in Next Generation Mobile Broadcasting , D. Gómez-Barquero Ed. Boca Raton, FL, USA: CRC Press, 2013, pp. 609-634. 


\section{Papers in conference proceedings (international and na- tional)}

[C1] D. Gozálvez, D. Vargas, D. Gómez-Barquero, and N. Cardona, "Performance evaluation of DVB-T2 time interleaving in mobile environments," Proc. IEEE Vehicular Technology Conference (VTC) Fall, Ottawa, Canada, September 2010.

[C2] D. Gómez-Barquero, D. Vargas, P. Gomez, J. Llorca, C. Romero, J. Puig, J. Gimenez, J. Lopez, D. Gozalvez, N. Cardona, "DVB-NGH, La Nueva Generacion de Television Digital Movil," Jornadas Telecom $I+D$, Santander, Spain, 2011.

[C3] D. Vargas, A. Winkelbauer, G. Matz, D. Gómez-Barquero and N. Cardona, "Low Complexity Iterative MIMO Receivers for DVB-NGH Using Soft MMSE Demapping and Quantized Log-Likelihood Ratios," 3rd MC and Scientific Meeting, European COST IC1004 action, Barcelona, Spain, February 2012.

[C4] S. Moon, W. Ko, D. Vargas, D. Gozálvez, M.D. Nisar, V. Pauli, "Enhanced Spatial Multiplexing for Rate-2 MIMO of DVB-NGH System," Proc. IEEE 19th International Conference on Telecommunications (ITC), Jounieh, Lebanon, April 2012.

\section{Contributions to standardization activities}

[S1] 9 contributions to the MIMO task force in the standardization process of DVB-NGH.

Contributions to the performance evaluation and validation of physical layer candidate technologies:

- MIMO precoding techniques based on rotation matrices in NGH mobile/portable channels,

- Performance evaluation of MIMO precoding techniques based on rotation matrices with real channel estimation in NGH mobile/portable channels,

- Performance evaluation of MIMO-BICM with iterative decoding and new parallel bit-interleaving permutations,

- Performance evaluation of MIMO-BICM with iterative decoding and MMSE demapping with prior information. 
[S2] 2 contributions to the DVB Technical Module MIMO Study Mission.

- Contributions to the capacity evaluation of multi-antenna schemes in fixed rooftop channel models for DTT systems. In particular, evaluation with the MIMO MGM channel model with log-normal fading.

[S3] 7 contributions to the TG3/S32-2 Ad-hoc Group on Modulation and Coding in the standardization process of ATSC 3.0.

Contributions for the performance evaluation and validation of physical layer candidate technologies:

- Bit-interleaving coded modulation components: LDPC codes, parallel bit-interleaving permutations and non-uniform constellations,

- Transmit diversity techniques for Multiple-Input Single-Output systems in SFN networks,

- Performance evaluation and optimization of MIMO precoding based on rotation matrices.

\section{Other journals not directly related to this thesis}

[OJ1] Y. J. D. Kim, J. Bajcsy, and D. Vargas, "Faster-Than-Nyquist Broadcasting in Gaussian Channels: Achievable Rate Regions and Coding," IEEE Transactions on Communications, vol. 64, no. 3, pp. 1016-1030, March 2016. 


\section{Chapter 2}

\section{Preliminaries}

$T^{\text {HIS CHAPTER describes fundamental concepts on wireless communications }}$

1 needed for the rest of the thesis. First, a system overview of main communication components is provided. The fundamentals of MIMO communications taking a look at the channel matrix representation, types of fading, gains provided by MIMO and information theoretic limits are presented. The fundamental trade-off in MIMO communication between spatial multiplexing (to increase the data rate) and signal diversity (to increase the signal resilience against channel fading) is presented. This fundamental trade-off serves as introduction to review two important transmission approaches, the so-called space-frequency/time-block codes (to extract diversity) and spatial multiplexing schemes (to extract the multiplexing gain). The focus is then given to practical systems based on bit-interleaved coded modulation that aim at extracting the various benefits of the MIMO channel. Followed by a description of the different components and their interactions, the information theoretic limits of MIMO-BICM systems are presented. The last part of this chapter is devoted to the receiver side. First it introduces the concept of iterative demapping that can provide significant system performance gains at the cost of higher receiver complexity. Additionally, an important aspect on practical receiver implementation is the quantization of the received information which permits signal processing with finite resolution. This chapter describes quantization concepts and design challenges. The theoretical and practical description of the communication components detailed in this chapter will allow to introduce with more in-depth the contributions of this thesis work. 


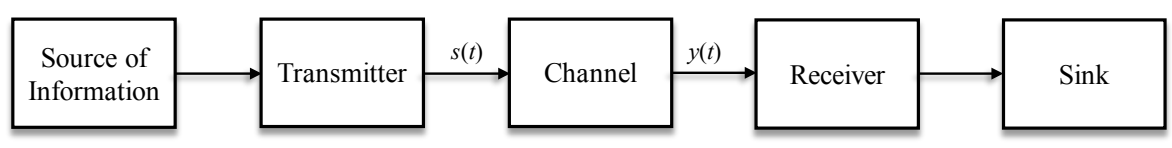

Figure 2.1: Simplified communication system block diagram.

\subsection{System Model Overview}

A basic communication system block diagram is presented in Fig. 2.1. A source generates information, generally in the form of bits, to be conveyed to other point of a communication link. This information is fed to a transmitter that adapts the information characteristics to the channel with the goal to maximize the transmission rate (i.e., number of information bits or symbols per second). A general representation of the continuous time baseband signal $s(t)$ is:

$$
s(t)=\sum_{n \in \mathbb{Z}} a[n] x(t-n T),
$$

where $a[n]$ are the information symbols which modulate bandlimited continuous time pulses $x(t+n T)$, where $n \in \mathbb{Z}$ (i.e., set of all integers). The transmitter sends information every $T$ seconds which produces an information rate of $1 / T$ $\mathrm{Hz}$ proportional to the signal bandwidth. The signal $s(t)$ is passed through the channel $h(t)$ which distorts its characteristics producing a signal:

$$
y(t)=h(t) * s(t)+w(t)
$$

where $w(t)$ is a white Gaussian random process and the operator * denotes the convolution. The receiver then has the task to recover the transmitted information. Assuming that the receiver samples the received signal $y(t)$ at every $0, T, 2 T, \ldots$ and considering that the Nyquist rate signalling criterion is fulfilled, the received signal can then be represented by the discrete time baseband model:

$$
y[n]=h[n] s[n]+w[n],
$$

where $n$ represents the $n$th sample. With the sampled received signal $y[n]$ the receiver corrects the corrupted information bits which are finally sent to the sink which completes the information loop. This provides a very simplified view of a communication system, now a closer look at the specific components at the transmitter, receiver and channel characteristics is provided.

Notation In the rest of the thesis the following notation is used: lower case letters $z$ denote scalars, boldface lower case letters $\mathbf{z}$ denote column vectors, boldface upper case letters $\mathbf{Z}$ denote matrices, the superscripts ${ }^{T},{ }^{\dagger}$ denote 


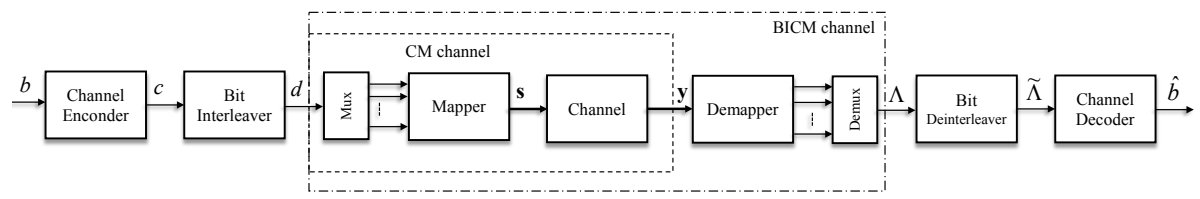

Figure 2.2: Basic transmit to receive bit-interleaved coded modulation system block diagram.

transposition and Hermitian transposition, the operator $E_{\mathbf{Z}}\{\cdot\}$ denotes the expectation over the random variable $\mathbf{Z},\|\cdot\|_{F}$ is the Frobenius norm, $|\mathcal{X}|$ is the cardinality of the set $\mathcal{X}$, and $N_{t}$ and $N_{r}$ are the number of transmit and receive antennas, respectively.

Fig. 2.2 presents a system block diagram based on the bit-interleaved codedmodulation architecture in the general case where multiple information streams are transmitted across multiple transmit antennas. In the following, details and a description of the functionalities and structure of each block in the chain is provided.

A bit stream $b$ that is formed by blocks of data of size $N$ which are input to the channel encoder is assumed. The channel encoder adds redundancy to increase the signal resilience against errors generated by the channel generating a block of coded bits of size $N_{c}$ (i.e., $N_{c} \geq N$ ). The ratio $N / N_{c}$ is the code-rate and as an example a code-rate $1 / 2$ means that half of the code word contains source information, while the other half contains parity data. In a BICM system the encoded bits $c$ are bit-interleaved by a permutation sequence $\pi$ of size $N_{c}$ which breaks the dependencies introduced by the channel encoder. In the case that multiple streams are accommodated to multiple transmit antennas, the interleaved coded bits are demultiplexed to $N_{t}$ streams by a bitwise multiplexer $T$. At the $l$ th stream, $l=1, \ldots, N_{t}$, groups of $m_{l}$ bits (where $m_{1}+\ldots+$ $m_{N_{t}}=m$ ) are mapped to complex symbols via a one-to-one mapping function $\mu \triangleq\{0,1\}^{m_{l}} \rightarrow \mathcal{X}$ were $\mathcal{X}$ is the set of possible constellation symbols with cardinality $|\mathcal{X}|=2^{m_{l}}$. Each of the constellation symbols at each of the transmit streams (or layers) form a vector $\mathbf{s}$ of size $N_{t} \times 1$ that is transmitted through the channel which distorts the signal and adds AWGN noise. The received signal vector $\mathbf{y}$ of dimension $N_{r} \times 1$ is passed to the demapper which provides soft information about the transmitted information bits $d$ in the form of loglikelihood ratios $\Lambda$. The stream $\Lambda$ is de-interleaved and passed to the channel decoder and outputs an estimate vector $\hat{b}$ of the transmitted bits $b$. More specific information of the BICM components will be given in Section 2.4 as well as its particular application in DTT systems. 


\subsection{MIMO Channel: Signal model, Gains and Capacity}

\subsubsection{AWGN channel}

Given the discrete complex base-band representation of (2.3), a received signal $y$ distorted by AWGN noise at the $n$th sample is:

$$
y[n]=s[n]+w[n],
$$

where $s[n]$ is the transmitted symbol and $w[n] \sim \mathcal{C N}\left(\mathbf{0}, \sigma^{2}\right)$ is additive circularly symmetric complex Gaussian noise where $\sigma^{2}$ is the noise power. This channel, although simple, provides with an accurate model for a wide set of communication channels. Shannon in his path-breaking paper [54] showed that the maximum rate at which information can be communicated across noisy channel with arbitrary reliability is:

$$
C_{\text {awgn }}=\log _{2}(1+\mathrm{SNR}) \text { bits } / \mathrm{s} / \mathrm{Hz}
$$

where SNR is the signal-to-noise ratio $P / \sigma^{2}$ where $P=\|s\|^{2}$ is the variance of the transmitted signal. As the transmission duration tends to infinity the probability of error asymptotically approaches zero as long as the rate of information $R<C_{\text {awgn }}$.

\subsubsection{Fading}

In the scenario with one transmit and one receive antennas where a transmitted signal $s$ is distorted due to the transmission through a wireless environment with multiple obstacles and signal reflections the discrete complex base-band model can be mathematically expressed as:

$$
y[n]=h[n] s[n]+w[n],
$$

where $h \sim \mathcal{C N}(\mathbf{0}, 1)$ is the fading coefficient at sample $n$. The amplitude $|h|$ is Rayleigh distributed and it models the arrival of a large number of rays at a singe receive antenna. At the receiver the value of $h$ can be estimated (under certain channel variability constrains) for coherent detection by tracking the channel with pilot information sent multiplexed with the data. In the case that the information of the channel estate $h$ is absent at the receiver this is known as blind or non-coherent detection. In this thesis it is always assumed that the receiver estimates the channel for coherent detection. 


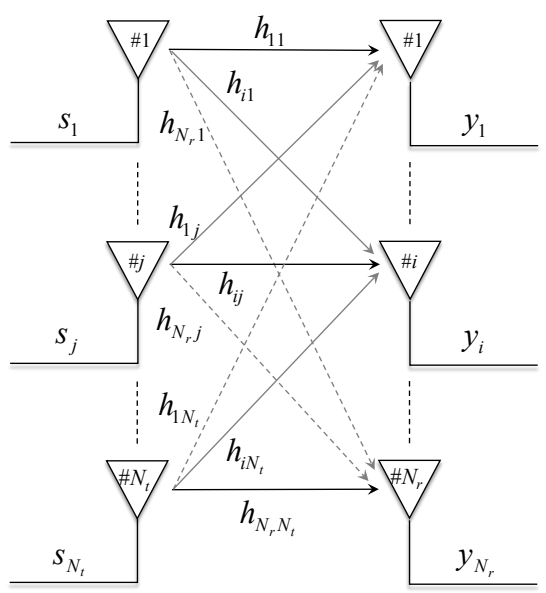

Figure 2.3: MIMO channel diagram.

Fast and Block Fading The type of fading can be classified as fast fading and block fading depending on the channel variability and the duration of the code words. First the channel coherence bandwidth/time is defined as the frequency/time range where the channel variation can be considered constant. Fast fading refers to the situation where within a code word multiple coherence periods can be observed, while for the block fading case the channel remains constant over the entire code word. Typically, fast fading occurs due to constructive and destructive interference at the receive terminals due to multipath interference or high speed mobility reception. Deep fades in fast fading operation can vanish received signal up to $40 \mathrm{~dB}$ [55]. For block fading, the fading can drop the received signal up to $20 \mathrm{~dB}$ [55], and it can be caused due to varying obstruction of the LoS between the transmitter and receiver, such as buildings or hilly terrain.

\subsubsection{Multiple Antenna Channel}

In the case that $N_{t}$ transmit antennas and $N_{r}$ receive antennas are used in the communication link, the discrete complex base-band model is:

$$
\mathbf{y}[n]=\mathbf{H}[n] \mathbf{s}[n]+\mathbf{w}[n]
$$

where $\mathbf{s}$ is the $N_{t} \times 1$ transmitted vector and $\mathbf{w} \sim \mathcal{C N}\left(\mathbf{0}, \sigma^{2} \mathbf{I}\right)$ is $N_{r} \times 1$ additive circularly symmetric complex Gaussian noise. In (2.7) $\mathbf{H}$ is the channel 
represented by a matrix of dimension $N_{r} \times N_{t}$ :

$$
\mathbf{H}=\left[\begin{array}{ccccc}
h_{11} & \ldots & h_{1 j} & \ldots & h_{1 N_{t}} \\
\vdots & \ddots & \vdots & \ddots & \vdots \\
h_{i 1} & \ldots & h_{i j} & \ldots & h_{i N_{t}} \\
\vdots & \ddots & \vdots & \ddots & \vdots \\
h_{N_{r} 1} & \ldots & h_{N_{r} j} & \ldots & h_{N_{r} N_{t}}
\end{array}\right]
$$

where $h_{i j}$ is the channel coefficient between the $i$ th receive antenna and the $j$ th transmit antenna and distributed -in the general case- as $\mathcal{C N}(\mathbf{0}, 1)$. The index $n$ is omitted for simplicity in the rest of the subsection. Fig. 2.3 shows a representation of a MIMO channel. Different antenna configurations are defined depending on the number of transmit $\left(N_{t}\right)$ and receive $\left(N_{r}\right)$ antennas. SISO refers to the case when a single antenna is used at both sides of the communication link, Single-Input Multiple-Output (SIMO) when only multiple antennas are employed at the receiver, MISO when only multiple antennas are used at the transmitter, and the general case when both the transmitter and the receiver have multiple antennas is called MIMO.

Channel normalization In this thesis the channel power is defined as the squared Frobenius matrix norm:

$$
\|\mathbf{H}\|_{F}^{2}=\sum_{i, j}^{N_{r}, N_{t}}\left|h_{i j}\right|^{2},
$$

and additionally we force $\sum_{i}^{N_{r}}\left|h_{i j}\right|^{2}=1$. As an example for a system with $N_{r}=$ 2 and $N_{t}=1$ the channel power in (2.9) is equal to 1 and for a MIMO system with $N_{r}=2$ and $N_{t}=2$ the channel power in (2.9) is equal to 2 . This channel normalization is valid for the antenna schemes considered in this dissertation with up to two receive antennas, and it will be useful when comparing MIMO channels with different structure (e.g., co-polar and cross-polar) for the same number of transmit and receive antennas.

SNR definition In the literature multiple definitions are used for the SNR. In this thesis the SNR is defined as $\mathrm{SNR}=P / \sigma^{2}$, where $P$ is the total transmitted power across all the transmit antennas. For a fair comparison between MIMO schemes with different number of transmit antennas, the following power constrain: $\sum_{i}^{N_{t}} P_{i}=1$ is set.

CNR definition In a OFDM system the SNR can be defined with respect to the CNR. In a practical OFDM signal some of the outer end carriers are unused 
(guard carriers) to reduce the potential interfere to adjacent RF channels due to the spectrum roll-off. The number of guard carriers depend on the FFT size, the larger the FFT-size the quicker the spectrum roll-off and therefore more carriers can be used to convey information data [45]. In the CNR definition used in this thesis the carrier power refers to information carrier power. Since the noise added at the receiver (in time domain) affects all the carriers (i.e., information and guard carriers) the signal to noise ratio at the input of the OFDM demodulator (SNR) and the output of the OFDM demodulator (CNR) has the following relationship in $\mathrm{dB}$ :

$$
\mathrm{SNR}=\mathrm{CNR}-10 \cdot \log _{10}\left(\frac{\mathrm{FFT}_{\text {size }}}{N_{\text {Info-carriers }}}\right) .
$$

Multi-antenna self interference At the $i^{\text {th }}$ receive antenna the signal is:

$$
y_{i}=\sum_{j}^{N_{t}} h_{i j} s_{j}+w_{i}, \quad i=1,2 \ldots, N_{r}
$$

as we can see the received signal at the $i$ th antenna is the combination of all the signals from all the transmit antennas plus the AWGN noise. Let us consider at the $j$ th receive antenna the symbol from the $i$ th transmit antenna when $i=j$ as the desired message and the other transmit symbols (i.e., $i \neq j$ ) as interference. Then expression (2.11) can be decomposed as:

$$
y_{i}=h_{i i} s_{i}+\underbrace{\sum_{j \neq i}^{N_{t}} h_{i j} s_{j}}_{\text {interfence term }}+w_{i}, \quad i=1,2 \ldots, N_{r} .
$$

Here, we can clearly observe the inter-antenna interference created in a MIMO channel. Optimal detection of the received vector $\mathbf{y}$ with the inter-antenna interference term increases the search space of the received signal to $|\mathcal{X}|^{N_{t}}$. The full space search increases exponentially with the number of the transmit antennas which can impose significant limitations on the system complexity for typical constellation sizes and number of transmit antennas. More detailed explanation of MIMO demodulation and complexity issues will be discussed in subsection 2.4.4.

MIMO Gains The implementation of MIMO provides array gain, diversity gain and multiplexing gains which are described next. Array gain increases the received SNR with multiple transmit and/or receive antennas. When channel state information is available at the receiver, the signal received from multiple receive antennas can be added coherently. In a transmitter with multiple 
transmit antennas and channel state information, the information at each of the transmit antennas can pre-processed so the signals add coherently at the receiver side. This is also known as beamforming, however, information about the channel state at the transmitter is absent in DTT systems. Diversity gain improves the reliability of the transmission by sending the same information through independently faded spatial branches to reduce the probability that all channels are in a deep fade. The simultaneous utilization of multiple antennas at the transmitter and receiver side permits the opening of multiple spatial pipes across the wireless channel providing multiplexing gain.

\subsubsection{MIMO Channel Capacity}

Fixed channel For a fixed matrix $H$, the channel capacity derived by Telatar [56] with information about the channel coefficients only available at the receiver is:

$$
C=\log _{2} \operatorname{det}\left(\mathbf{I}_{N_{r}}+\frac{\mathrm{SNR}_{N_{t}}}{\mathbf{H H}^{\dagger}}\right),
$$

where SNR is the received SNR at each receive antenna.

An alternative formulation for the channel capacity comes from the singular value decomposition of a matrix [57]

$$
\mathbf{H}=\mathbf{U} \boldsymbol{\Sigma} \mathbf{V}^{\dagger},
$$

where $\mathbf{U}$ and $\mathbf{V}$ are $N_{r} \times N_{r}$ and $N_{t} \times N_{t}$, respectively, unitary matrices and $\Sigma$ of size $N_{r} \times N_{t}$ is a rectangular matrix with non-negative real numbers on the diagonal. Substituting (2.14) in (2.7) and defining [13]:

$$
\begin{aligned}
\tilde{\mathbf{x}} & :=\mathbf{V}^{\dagger} \mathbf{x} \\
\tilde{\mathbf{y}} & :=\mathbf{U}^{\dagger} \mathbf{y} \\
\tilde{\mathbf{w}} & :=\mathbf{U}^{\dagger} \mathbf{w}
\end{aligned}
$$

expressions (2.7) can be reformulated as:

$$
\tilde{\mathbf{y}}=\Sigma \tilde{\mathbf{x}}+\tilde{\mathbf{w}} .
$$

Using the fact that $\tilde{\mathbf{w}}$ is the equally distributed ${ }^{1}$ as $\mathbf{w},(2.13)$ is expressed as a function of the singular values of the channel matrix $\mathbf{H}$ :

$$
C=\sum_{i=1}^{n_{\min }} \log _{2}\left(1+\frac{\mathrm{SNR}}{N_{t}} \lambda_{i}^{2}\right),
$$

where $\lambda_{i}$ is the $i$ th diagonal entry of $\Sigma$.

\footnotetext{
${ }^{1} \mathbf{U w} \sim \mathbf{w}$ if $\mathbf{w} \sim \mathcal{C N}(\mathbf{0}, \mathbf{I})$ and $\mathbf{U}$ is unitary matrix, i.e., $\mathbf{U}^{\dagger} \mathbf{U}=\mathbf{I}$.
} 
The above expression shows that the MIMO channel can be decomposed into a parallel channel where the gain of each channel is given by the singular values of the matrix $\mathbf{H}$. The value $n_{\min }$ is the number of non-zero singular values which depend on the rank of the matrix channel $\mathbf{H}$. The higher the value $n_{\text {min }}$ the higher the number of parallel channels in which information can be transmitted through. The maximum capacity in a MIMO channel is achieved when the matrix $\mathbf{H}$ is full rank, i.e., columns are linearly independent (when $N_{r}>N_{t}$ ) or rows are linearly independent (when $N_{t}>N_{r}$ ). Full rank matrices are achieved in environments with many scatters with sufficient separation between antennas, or in MIMO systems that use different polarizations. In the extreme case that the MIMO components are fully correlated, the channel matrix $\mathbf{H}$ becomes singular, with only one non-zero singular value, which has the same capacity as a SISO communication link. The impact of correlation in MIMO capacity will be studied in more detail in Chapter 3 and Appendix A.

Fast fading channel If the channel matrix $\mathbf{H}$ is a Rayleigh random variable i.i.d. from sample to sample (i.e., fast fading) the long-term rate of reliable communication is:

$$
C=E_{\mathbf{H}}\left\{\log _{2} \operatorname{det}\left(\mathbf{I}_{N_{r}}+\frac{\mathrm{SNR}}{N_{t}} \mathbf{H} \mathbf{H}^{\dagger}\right)\right\} .
$$

This channel capacity commonly known as ergodic-capacity is achieved for systems where coding is performed over multiple channel realizations. This is the case as in mobile channels where the information can be interleaved over different channel realizations due to the varying nature of the mobile channel. Although the ergodic capacity is achieved for infinite long code-words, practical systems have sufficiently long coding and interleaving which makes the ergodic-capacity a valuable metric. The capacity in (2.18) is for the case where the receiver perfectly tracks the channel states but no knowledge is available at the transmitter site. In this channel the optimal transmission strategy is to isotropically transmit among the transmit antennas. The case with channel state information at the transmitter is discussed in a latter section.

Block fading channel In the situation of slow fading, where a channel realization does not vary within a code-word, the capacity limit as the maximum achievable rate with arbitrary reliability is no longer useful. In this case, since there is no average (or interleaving) over multiple channel realizations, the probability that a specific channel realization is

$$
\log _{2} \operatorname{det}\left(\mathbf{I}_{N_{r}}+\frac{\mathrm{SNR}}{N_{t}} \mathbf{H} \mathbf{H}^{\dagger}\right)<R
$$


is no longer zero. In this case it is said that the channel is in outage and is useful to compute the probability of outage given the channel distribution:

$$
p_{\text {out }}=\mathbb{P}\left\{\log _{2} \operatorname{det}\left(\mathbf{I}_{N_{r}}+\frac{\mathrm{SNR}}{N_{t}} \mathbf{H H}^{\dagger}\right)<R\right\} .
$$

With the outage probability $p_{\text {out }}$, one can define the $\epsilon$-outage-capacity $C_{\epsilon}$ which is the largest rate of transmission $R$ such $p_{\text {out }}$ is less than $\epsilon$. It can be expressed mathematically as:

$$
C_{\epsilon}=\sup \left\{R \mid p_{\text {out }}<\epsilon\right\}
$$

Channel state information at the transmitter side In the case that the transmitter has also knowledge of the information about the channel state, the transmission can be adapted to optimize the system performance. This case is referred as Channel Transmit State Information (CTSI).

Similarly to the case with CSI only at the receiver (i.e., Channel Receive State Information (CRSI)), the analysis is started with the simple case when the channel matrix $\mathbf{H}$ is fixed. In this case, the capacity expression in (2.17) can be rewritten as:

$$
C=\sum_{i=1}^{n_{\min }} \log _{2}\left(1+\frac{P_{i}^{*}}{\sigma^{2}} \lambda_{i}^{2}\right),
$$

where $P_{i}^{*}$ are the optimized powers with the water-filling algorithm [13] assigned to each of equivalent MIMO channels which follow the following expression:

$$
P_{i}^{*}=\max \left\{\mu-\frac{\sigma^{2}}{\lambda_{i}^{2}}, 0\right\},
$$

and with the common power constrain $\sum_{i} P_{i}^{*}=1$.

In the fast fading case, the ergodic capacity is:

$$
C=\sum_{i=1}^{n_{\min }} E\left\{\log _{2}\left(1+\frac{P_{i}^{*}}{\sigma^{2}} \lambda_{i}^{2}\right)\right\}
$$

For the slow-fading case:

$$
p_{\text {out }}=\mathbb{P}\left\{\sum_{i=1}^{n_{\text {min }}} \log _{2}\left(1+\frac{P_{i}^{*}}{\sigma^{2}} \lambda_{i}^{2}\right)<R\right\} .
$$

It is noted that for the slow-fading case, transmission can only be optimized in the spatial domain, since the transmitter does not have knowledge of the variations of the channel in time. 
In the situation that the transmitter wants to optimize the transmission in a statistical way, one has to find the optimal transmit covariance matrix $\mathbf{Q}$ that maximizes the following problem:

$$
\begin{aligned}
& \underset{\mathbf{Q} \succeq 0 \text { s.t. }}{\operatorname{maximize}} \quad E_{\mathbf{H}}\left\{\log _{2} \operatorname{det}\left(\mathbf{I}_{N_{r}}+\frac{\rho}{N_{t}} \mathbf{H} \mathbf{Q} \mathbf{H}^{\dagger}\right)\right\} . \\
& \operatorname{trace}(\mathbf{Q})=N_{t}
\end{aligned}
$$

This will be further developed in Chapter 3, where a precoder is optimized based on the statistics of the channel.

\subsection{A Fundamental Trade-Off and Transmission Approaches}

\subsubsection{Multiplexing-Diversity Trade-Off}

A MIMO system can be used to increase the transmission reliability through diversity gain or to increase the system rate through spatial multiplexing gain. There is however, a trade-off between the diversity and multiplexing gain that may be achieved in a MIMO channel $[13,58]$, i.e., one cannot arbitrarily increase the diversity and multiplexing gains at the same time.

For a MIMO system with a fixed transmission rate $R$, the maximum achievable diversity gain $d_{\max }$ is given by the negative slope of the probability of error $p_{e}(\mathrm{SNR}, R)$ (which is a function of the rate $R$ and SNR) with asymptotic SNR [59]:

$$
d_{\max }=-\lim _{\mathrm{SNR} \rightarrow \infty} \frac{\log _{2}\left(p_{e}(\mathrm{SNR}, R)\right)}{\log _{2}(\mathrm{SNR})} .
$$

In such a system the probability of error scales as $p_{e}(\mathrm{SNR}, R) \approx \mathrm{SNR}^{-d_{\max }}$. For instance, in a MIMO slow fading i.i.d. Rayleigh channel with $N_{t}$ transmit and $N_{r}$ receive antennas, a capacity achieving system (with optimal transmission and receiver detection) can achieve a maximum diversity order $d_{\max }=N_{t}$. $N_{r}[13,59]$.

Alternatively, the $N_{r} \times N_{t}$ MIMO system has a maximum multiplexing gain $r_{\max }$ given by the slope of the outage capacity with asymptotic SNR [59]:

$$
r_{\max }=\lim _{\mathrm{SNR} \rightarrow \infty} \frac{C_{\epsilon}(\mathrm{SNR})}{\log (\mathrm{SNR})} .
$$

In a MIMO slow fading i.i.d. Rayleigh channel and a capacity achieving system (with optimal transmission and receiver detection) the maximum multiplexing gain is $r_{\max }=\min \left\{N_{t}, N_{r}\right\}[59]$. 
Expressions in (2.27) and (2.28) are the two extremes of the so-called Diversity-Multiplexing Tradeoff (DMT) which describes the relationship between diversity and multiplexing in MIMO systems. A MIMO system cannot increase both the transmission rate and reliability to the maximum values in (2.27) and (2.28) but needs to trade between them. In a slow fading i.i.d. Rayleigh channel, the optimal DMT is a piecewise linear function:

$$
d(r)=\left(N_{t}-r\right)\left(N_{r}-r\right),
$$

where $r=0, \ldots, r_{\max }$. This trade-off indicates that if the rate is increased as $r \log _{2}(\mathrm{SNR})$, then the error probability decreases as SNR ${ }^{-d(r)}$ [13]. The design of MIMO coding schemes with different diversity and multiplexing capabilities has been an active area of research in the last two decades.

\subsubsection{Space-Frequency Block codes}

Coding the information symbols across the signal dimensions, i.e., space, time and frequency, can bring substantial performance improvements in the link reliability and throughput. Space Frequency (or Time) Block Codes (SFBC/STBC) code information data across space and frequency (or time) to get closer to theoretical performance limits. SFBC/STBC can be designed to increase transmission reliability, to increase the data rate, or both following the DMT principle. In order to exploit the benefits of SFBCs, it is necessary to employ orthogonal pilot patterns between antennas, so that the receiver can estimate the channel response from each transmit antenna. This means that to maintain the same resolution of channel estimation as with single antenna transmissions, the number of pilots required with one transmit antenna shall be doubled/quadrupled with SFBC across two/four transmit antennas. This additional overhead can impose significant limits in the final system performance [14].

A very well known STBC is the Alamouti code designed to provide full diversity with rate 1 (i.e., one information symbol per channel use) in a MISO $2 \times 1$ channel. The encoding matrix of the Alamouti code is:

$$
\mathbf{C}_{\text {Alam }}=\left[\begin{array}{cc}
c_{1} & -c_{2}^{\dagger} \\
c_{2} & c_{1}^{\dagger}
\end{array}\right]
$$

where $c_{1}$ and $c_{2}$ are constellation symbols. The columns of the matrix correspond to different transmit antennas and the rows correspond to different time or frequency slots. The Alamouti code is optimal in the sense that reaches the DMT frontier. With two receive antennas (i.e., $2 \times 2$ MIMO) the Alamouti code still provides full diversity but it is no longer optimal in terms of the DMT limit since only one information symbol is transmitted in one channel use. 


\subsection{A Fundamental Trade-Off and Transmission Approaches}

\section{Transmit Diversity Techniques in DTT systems}

The Alamouti code has been adopted in DTT systems first in DVB-T2 and then in DVB-NGH. In DVB-T2 it was used to encode information symbols from distributed pairs of transmitters to improve reception in SFNs where the signals from multiple transmitters can create destructive interference erasing parts of the received signal bandwidth. For optimal performance of SFBCs/STBCs with linear decoding complexity, it is important that channel responses for all the carriers (or time slots) carrying one block should remain unchanged. For this reason, the Alamouti code in DVB-T2 encodes information symbols in space and OFDM carriers since it was assumed that the channel coefficients in adjacent OFDM carriers have lower variation than in adjacent OFDM symbols.

Alternatively to Alamouti code, the performance in SFNs with single antenna receivers can be improved by applying a linear pre-distortion to the common waveform of the signals transmitted by the multiple sites increasing the spatial diversity. DVB-NGH adopted eSFN [60], a linear pre-distortion to the frequency carriers of the OFDM waveform in a manner that special signal processing at the receivers is not necessary, since the frequency pre-distortion is seen by the receivers as part of the channel. Compared to the Alamouti code, there is no additional overhead due to increased pilot density. Similarly, ATSC 3.0 has adopted an antenna scheme to improve the overall performance in SFN, known as Transmit Diversity Code Filter Sets (TDCFS) [61], but with better correlation properties than eSFN.

SFBCs can be also designed to provide multiplexing gain, a full-rate fulldiversity scheme for $2 \times 2$ MIMO channels is the Golden-Code proposed in [62] Its encoding matrix is:

$$
\mathbf{C}_{\mathrm{GC}}=\frac{1}{\sqrt{5}}\left[\begin{array}{cc}
\alpha\left(c_{1}+c_{2} \theta\right) & \bar{\alpha}\left(c_{1}+c_{2} \bar{\theta}\right) \\
i \bar{\alpha}\left(c_{3}+c_{4} \bar{\theta}\right) & \alpha\left(c_{3}+c_{4} \theta\right)
\end{array}\right],
$$

where $\theta=(1+\sqrt{5}) / 2, \bar{\theta}=(1-\sqrt{5}) / 2, \alpha=1+i(1-\theta), \bar{\alpha}=1+i(1-\bar{\theta})$, and $c_{i}, i=1, \ldots, 4$ are constellation symbols. The Golden-Code achieves the DMT limit with an encoding matrix providing nonvanishing determinant with decoding complexity proportional to $\mathcal{O}\left(\mathcal{X}^{4}\right)$. The Golden-Code was evaluated during the standardization process of DVB-NGH but was discarded in favour of more simple schemes such as plain SM due to the increased decoding complexity (decoding complexity of SM proportional to $\mathcal{O}\left(\mathcal{X}^{2}\right)$ ) and performance degradation when connected with strong channel coding such as the LDPC codes at low code-rates [29, 63].

Various SFBCs rate 1 and rate 2 codes for MIMO systems in distributed scenarios were also proposed and evaluated during the DVB-NGH standardization process for the hybrid terrestrial-satellite MIMO profile with multiple antennas at the terrestrial site and/or the satellite [32, 64, 65, 66, 67]. For rate 
1, the codes adopted in the hybrid terrestrial-satellite MIMO profile depend on the number of transmit antennas at the terrestrial site and satellite. With one transmit antenna at the terrestrial site and the satellite, the adopted codes are based on the Alamouti code and eSFN. With two transmit antennas at the terrestrial site and one transmit antenna at the satellite, or vice-versa, the adopted codes are based on the Alamouti+QAM code [67] and eSFN. In the case that the terrestrial site and the satellite have two transmit antennas, the adopted codes are based on the Alamouti code and eSFN. For rate 2, the codes adopted in the hybrid terrestrial-satellite MIMO profile are based on rotation matrices which will be presented in latter sections.

\subsubsection{Spatial Multiplexing Techniques}

In the previous subsections, SFBC schemes that increase the multiplexing rate of the communication system have been presented. However, the most simple way of using spatial multiplexing is by sending independent information across the transmit antennas, i.e., MIMO SM. In a $2 \times 2$ MIMO system with SM, one information symbols is transmitted by one antenna and received by two antennas through two spatial paths. The spatial diversity of MIMO SM can be increased by applying a rotation matrix $\mathbf{R}$ to the data streams:

$$
\mathbf{R}=\left[\begin{array}{cc}
\cos \theta & \sin \theta \\
\sin \theta & -\cos \theta
\end{array}\right] .
$$

A $2 \times 2$ rotation matrix has the form in $(2.32)$ and is a family of matrices that are characterized as orthonormal matrices, i.e., $\mathrm{RR}^{T}=\mathbf{I}$. A rotation matrix with parameter $\theta$ linearly combines independent data streams so each transmit antenna conveys information of all data streams increasing the spatial diversity. The input-output relationship of a processing block based of a rotation matrix is:

$$
\left[\begin{array}{l}
y_{1} \\
y_{2}
\end{array}\right]=\left[\begin{array}{cc}
\cos \theta & \sin \theta \\
\sin \theta & -\cos \theta
\end{array}\right]\left[\begin{array}{l}
x_{1} \\
x_{2}
\end{array}\right]=\left[\begin{array}{c}
\cos \theta \\
\sin \theta
\end{array}\right] x_{1}+\left[\begin{array}{c}
\sin \theta \\
-\cos \theta
\end{array}\right] x_{2} .
$$

It compares with the case where no rotation matrix is applied with inputoutput relationship:

$$
\left[\begin{array}{l}
y_{1} \\
y_{2}
\end{array}\right]=\left[\begin{array}{ll}
1 & 0 \\
0 & 1
\end{array}\right]\left[\begin{array}{l}
x_{1} \\
x_{2}
\end{array}\right]=\left[\begin{array}{l}
1 \\
0
\end{array}\right] x_{1}+\left[\begin{array}{l}
0 \\
1
\end{array}\right] x_{2}
$$

It can be seen that the information vector $\left[x_{1}, x_{2}\right]^{T}$ is transmitted over the basis vectors $[1,0]^{T}$ and $[0,1]^{T}$ for the non-rotated case, and $[\cos \theta, \sin \theta]^{T}$ and $[\sin \theta,-\cos \theta]^{T}$ for the rotated case. 

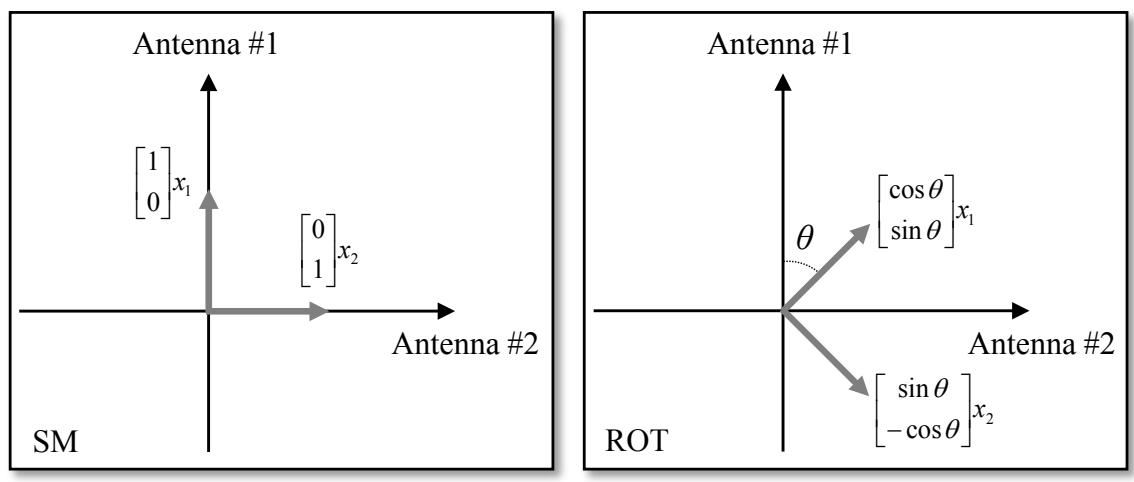

Figure 2.4: Vector representation of a MIMO system with two transmit antennas with (right) and without (left) rotation matrix. Without rotation the symbols in each data stream are transmitted in just one antenna each, while with rotation the symbols in each data stream are transmitted through both antennas.

Fig. 2.4 illustrates the vector representation of a $2 \times 2$ MIMO system transmitting information symbols $x_{1}$ and $x_{2}$ in a scheme without the application of a rotation matrix (left diagram labelled with SM) and with the application of a rotation matrix (right diagram labelled with ROT). In the case of no rotation, each data symbol $x_{1}$ and $x_{2}$ are allocated directly to a single transmit antenna. For the case of rotation, both symbols $x_{1}$ and $x_{2}$ are allocated to both transmit antennas.

The following example compares the received signals with and without rotation matrix processing at the transmitter in the situation when the signal from one of the transmit antennas in a MIMO channel is lost due to a deep fading realization.

Example: Received symbols in a communication system with a $2 \times 2$ MIMO channel in the event of signal outage from one of the transmit antennas.

The input-output signal relationship for the non-rotated case can be expressed as:

$$
\left[\begin{array}{l}
r_{1} \\
r_{2}
\end{array}\right]=\underbrace{\left[\begin{array}{ll}
h_{11} & h_{12} \\
h_{21} & h_{22}
\end{array}\right]}_{\text {channel }} \underbrace{\left[\begin{array}{ll}
1 & 0 \\
0 & 1
\end{array}\right]}_{\text {linear-transf. }}\left[\begin{array}{l}
x_{1} \\
x_{2}
\end{array}\right],
$$


and the output symbols $r_{1}$ and $r_{2}$ are (the noise is neglected in this example to ease the notation):

$$
\begin{gathered}
r_{1}=h_{11} x_{1}+h_{12} x_{2} \\
r_{2}=h_{21} x_{1}+h_{22} x_{2} .
\end{gathered}
$$

The input-output signal relationship for the rotated case can be expressed as:

$$
\left[\begin{array}{l}
r_{1} \\
r_{2}
\end{array}\right]=\underbrace{\left[\begin{array}{ll}
h_{11} & h_{12} \\
h_{21} & h_{22}
\end{array}\right]}_{\text {channel }} \underbrace{\left[\begin{array}{cc}
\cos \theta & \sin \theta \\
\sin \theta & -\cos \theta
\end{array}\right]}_{\text {linear-transf. }}\left[\begin{array}{l}
x_{1} \\
x_{2}
\end{array}\right],
$$

and the output symbols $r_{1}$ and $r_{2}$ are:

$$
\begin{gathered}
r_{1}=h_{11}\left(x_{1} \cos \theta+x_{2} \sin \theta\right)+h_{12}\left(x_{1} \sin \theta-x_{2} \cos \theta\right) \\
r_{2}=h_{21}\left(x_{1} \cos \theta+x_{2} \sin \theta\right)+h_{22}\left(x_{1} \sin \theta-x_{2} \cos \theta\right) .
\end{gathered}
$$

Assuming the signal from the first transmit antenna is lost, the first column of the $2 \times 2$ MIMO channel matrix is the zero vector, i.e., $\left[h_{11}, h_{21}\right]^{T}=$ $[0,0]^{T}$. The received symbols for the non-rotated case are:

$$
\begin{gathered}
r_{1}=h_{12} x_{2} \\
r_{2}=h_{22} x_{2},
\end{gathered}
$$

and for the rotated case are:

$$
\begin{gathered}
r_{1}=h_{12}\left(x_{1} \sin \theta-x_{2} \cos \theta\right) \\
r_{2}=h_{22}\left(x_{1} \sin \theta-x_{2} \cos \theta\right) .
\end{gathered}
$$

One can observe that with the use of rotation matrices the two transmitted symbols $x_{1}$ and $x_{2}$ are received even in the case of the signal of one of the transmitters is in deep fade. Although this situation may appear as beneficial in all situations, the situation may differ when combined with FEC schemes. The performance of rotation matrices with FEC will be addressed in Chapter 3 of this thesis.

It is noted that to demodulate an SM signal it is necessary to employ orthogonal pilot patterns (as for the Alamouti code) between antennas, so that each receive antenna can estimate the channel response from each transmit antenna. Compared with single antenna transmissions, the required number of transmitted pilots must be doubled for the same resolution of channel estimation, hence reducing the overall spectral efficiency. 


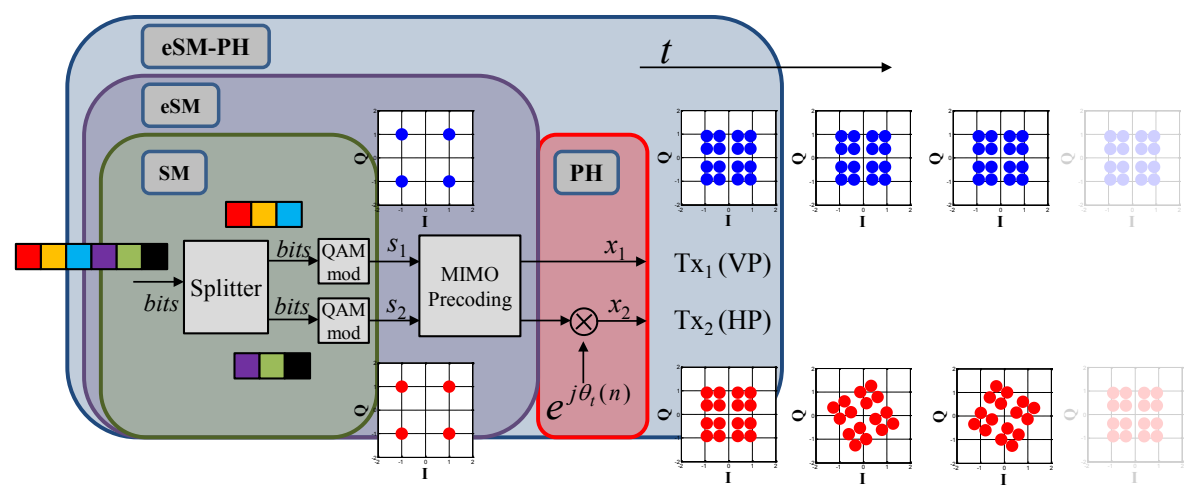

Figure 2.5: MIMO precoding scheme adopted in DVB-NGH form by a concatenation of rotation matrix and phase-hopping term with two transmit antennas with vertical polarization (VP) and horizontal polarization (HP).

\section{Spatial Multiplexing Techniques in DTT systems}

DVB-NGH and ATSC 3.0 have adopted a spatial multiplexing techniques based on rotation matrices and presented in Fig. 2.5. The presence of correlation in the MIMO channel due to LoS channel condition, which frequently happens in fixed terrestrial broadcast environments, is especially detrimental for MIMO SM. MIMO precoding based on rotation matrices overcomes this situation retaining the multiplexing capabilities of MIMO SM, and at the same time, increasing the robustness against spatial correlation. The information symbols are weighted and combined before their transmission across the antennas according to a specified rotation angle. This rotation angle can be tuned for different transmission parameters and channel conditions. In addition, a periodical phase hopping term is added to the second antenna in order to randomize the code structure and avoid the negative effect of certain channel realizations. The specific parameters and structure of the MIMO precoding inlcuded in DVB-NGH and ATSC 3.0 is detailed in Chapter 3. The analysis of MIMO precoding based on rotation matrices, with both an informationtheoretic point of view and with numerical evaluations using channel coding is also provided in Chapter 3. 


\subsection{MIMO-BICM: Components and Mutual In- formation}

A coded modulation system jointly designs modulation and coding. Trelliscoded modulation [39] and multilevel codes [40] are popular coded modulation schemes. In bit-interleaved coded modulation system [37, 38], modulation and coding are separated by a bit-interleaving allowing independent design of each component. However, the introduction of a bit-interleaver imposes an information loss due to the elimination of the interdependences of the transmitted coded bits. Design flexibility and robustness against fading channels have motivated rapid extensions to MIMO systems and its combination with OFDM modulation. Modern wireless communications systems, such as DVB-NGH and ATSC 3.0 include MIMO-BICM as key technology to cope with the increasing demand for data rate and transmission reliability.

In the next subsection introduces and details the main characteristics of the components of a MIMO-BICM system, and provide information-theoretic results of BICM and CM under fading channels.

\subsubsection{Forward Error Correction}

Channel coding is a core part of any communication systems which allows the correction of channel impairments in the received signal and is generally referred as Forward Error Correction (FEC). The information bits act as an input to the channel encoder which adds redundancy to the information bits to increase the robustness of the transmitted signal, allowing correction of corrupted bits at the receiver with feasible decoding complexity. Shannon in 1948 [54] derived the channel capacity as upper bound on the maximum reliable information rates achievable over noisy channels, which has become a practical benchmark for reliable communications over many practical communication channels. It was only with the development of practical error correcting codes such as the turbo-codes [68] and the LDPC codes [10, 69], that channel coding started to perform closely to the capacity limits of practical channels [70]. A concatenation of Bose-Chaudhuri-Hocquenghem (BCH) and LDPC codes has been adopted amongst others in the physical layers of DVB-T2, DVBNGH and ATSC 3.0. Compared with the FEC scheme used in first-generation DVB systems based on the concatenation of a convolutional and Reed-Solomon codes, the utilization of $\mathrm{BCH}$ and LDPC codes provide gains ranging from $3 \mathrm{~dB}$ to more than $5 \mathrm{~dB}$ for low and high bit-rate services, respectively, in stationary channels [71].

Low density parity check codes are linear, binary block codes and are constructed with sparse parity-check matrices which provide decoding complexity 
only increasing linearly with the size of the code. A sparse $\left(N_{c}-N\right) \times N_{c}$ paritycheck matrix $H$ (being $N$ and $N_{c}$ the size of the input uncoded bits and output coded bits, respectively) can be represented by factor graphs [72] consisting on $N_{c}$ variable nodes and $N_{c}-N$ check nodes (or number of parity-check equations). An entry value of 1 at the $i j$ th position of the matrix connects by an edge the $i$ th check node to the $j$ th variable node. Classical decoding consists on exchange of extrinsic soft information between the variable nodes and the check nodes in an iterative fashion until the estimated code-word verifies the parity-check equations (i.e., $H x^{T}=0^{T}$ ) or a maximum number of iterations is reached. This type of algorithm approximates Maximum a Posteriori (MAP) decoding performance and is termed belief propagation [73]. Although the decoding performance improves with increasing number of iterations, it comes at the expense of higher computational complexity. Furthermore, reaching a certain number of iterations, the performance improvement saturates and additional iterations only provide marginal improvement. A common value of maximum number of iterations is 50 , and is the one assumed for DTT systems such as DVB-T2 [45], and is the value used in this dissertation.

DVB-T2 and ATSC 3.0 specify code sizes of 16200 bits (short codes) and 64800 bits (long codes). DVB-NGH targeting mobile devices with limited hardware requirements specify up to 16200 bits code size. With the objective of reaching varying reception environments with services requiring different spectral efficiencies, modern DTT systems include in its specifications a wide set of code-rates, ranging from very low code-rates (for high robustness) to high code-rates (for maximum spectral efficiency). The MIMO profile of DVB-NGH defines 7 code-rates from $5 / 15$ to $11 / 15$ with equal spacing. ATSC 3.0 widens this set with a total of 13 code-rates from $2 / 15$ to $13 / 15$.

\subsubsection{Bit-Interleaver}

The output of the channel encoder is bit-interleaved to break the dependences introduced by the binary channel code and fed to the mapper. The bit interleaver permits an independent design of binary channel encoders and mapper, which can be adapted to the channel and system requirements, greatly simplifying the design. Although in the literature the bit-interleaver is generally defined as a sufficiently long random sequence, practical systems require the design of particular permutation sequences known at the transmitter and at the receiver.

Due to the irregular structure of the LDPC codes included in the DVB-T2 specification (and inherit by DVB-NGH) with non-uniform protection along the code-word and non-uniform robustness amongst the bits in the label of a multilevel constellation, a concatenation of a bit-interleaver and demux is introduced. This concatenation provides equal protection along the code-word 
and avoids performance degradation in fading channels. The design of the different stages of this type of bit-interleaver is a function of the constellation size, code-rate and channel encoding code-word size. The bit-interleaver part is comprised of a parity-interleaver followed by a column-twist block interleaver. The parity-interleaver applies a permutation sequence in such a way that the parity part of the parity-check matrix has the same structure as the information part. The parity-interleaved bits are serially written into the column-twist interleaver column-wise, and serially read out row-wise where the write start position of each column is twisted depending on the constellation size. Compared to this type of bit-interleaver, the MIMO profile of DVB-NGH includes in its specification a novel parallel bit-interleaver with reduced hardware complexity and low latency. The parallel bit-interleaver encourages the use of iterative structures and it has been optimized for iterative reception. The main novelty of this type of bit-interleaver is the inclusion of a group-wise interleaver which allows optimum combination between code bits and constellation symbols with different permutations for each combination of constellation and code-rate. Due to the additional performance and hardware benefits exhibit by this type of bit-interleaving, ATSC 3.0 has adopted this architecture as bit-interleaving solution.

\subsubsection{Mapping from Bits to Constellation Symbols}

The mapper takes in a bit-interleaved stream of coded bits in groups of $m$ bits and produces output complex valued symbol vector from a constellation set $\mathcal{X}$ with cardinality $|\mathcal{X}|=2^{m}$. The location of the constellation points has a direct impact in the system performance. Quadrature amplitude modulation (QAM) constellations are widely used in practical systems such as DVB-T, DVB-T2 and DVB-NGH. This constellation have uniform distribution of the symbols on the complex I\&Q plane with the same distance between any two adjacent points and have a squared shape. Fig. 2.6 shows an example of a 16QAM constellation. However, this uniform sampling of constellation points is suboptimal from the capacity point of view since to approach the Shannon informationtheoretic limit the transmitted signal has to be Gaussian distributed. Nonuniform constellations are designed to overcome the performance degradation due to uniform spaced constellations obtaining more Gaussian-like distributed constellations sets $[74,75,76]$. This gain is normally referred as shaping gain. Fig. 2.6 shows an example of a non-uniform 16-point constellation. Nonuniform constellations have been adopted in broadcasting standards such as DVB-NGH [77] and ATSC 3.0 [78]. Two types of non-uniform constellations have been defined: One-dimensional Non-Uniform Constellation (1D-NUC) and Two-dimensional Non-Uniform Constellation (2D-NUC). 1D-NUCs are formed by two non-uniform Pulse Amplitude Modulation (PAM)s, corresponding to 

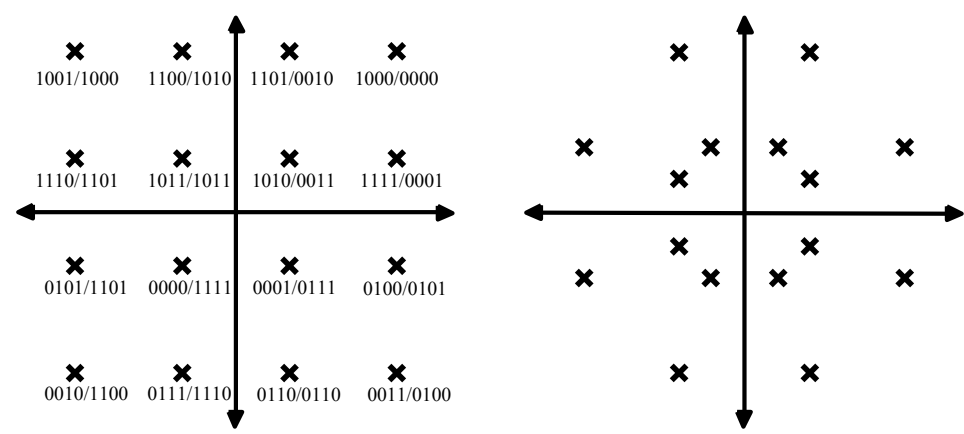

Figure 2.6: Constellation examples with 16 points. Left curves present 16QAM constellation with set-partitioning/Gray mappings. Right curve presents a two-dimensional non-uniform 16-point constellation.

the I and Q components, producing a non-uniform constellation with squared shape which allows the use of low complexity one-dimensional demappers [79]. 2D-NUCs optimize the positions of the constellation symbols in the complex I\&Q plane, producing a constellation with non-squared shape outperforming 1D-NUCs but with higher demapping complexity [80]. Non-uniform constellation in these systems have been optimized for single antenna transmissions. The performance of non-uniform constellations with cross-polar MIMO in multipath channels has been evaluated in [81]. In DVB-NGH, although included in the specification, 1D-NUCs were never evaluated in connection with MIMO. For ATSC 3.0 the same SISO-optimized constellations have been reused for the MIMO profile. The design of non-uniform constellations optimized for MIMO channels in terrestrial broadcasting systems is still an open research topic and is therefore out of the scope of this thesis. Hence, this dissertation will focus on the case of QAM constellations.

Besides of the constellation shape, the mapping of the $m$-length binary label to the set $\mathcal{X}$ with $2^{m}$ constellation symbols has an important impact in the performance of the BICM system. A popular mapping is the Gray labelling which has the property that any the bit-labels of any two adjacent constellation symbols only differ by one bit, i.e., the Hamming distance is at most one bit. In the high SNR regime Gray labelling minimizes the bit-error-probability [82]. In the context of BICM with iterative demodulation (see section 2.4.4) other mappings such as set-partitioning provide higher iterative demodulation gains in BICM systems [83]. An example of Gray and set-partitioning labellings is shown in Fig. 2.6. This dissertation will consider Gray labelling as the primary case as it is the selected mapping in broadcasting standards such as DVB-T2 and DVB-NGH. 


\subsubsection{MIMO Demodulation and Iterative Decoding}

In a MIMO coded system, joint demodulation and decoding achieves optimal error-rate performance for CM. Joint demodulation and decoding is able to exploit the bit interdependences introduced by the channel code. However, optimal performance comes at expense of prohibitively high computational complexity. In contrast, in BICM, demodulation and decoding are separated into independent processing blocks by using bit-wise interleaving with feasible computational complexity. Solving both tasks separately introduces an information loss because bit interdependences introduced by the channel code are no longer exploited. Although single antenna BICM systems with Gray labeling are able to perform close to CM capacity on AWGN channel, there is a significant gap between MIMO demodulation in a BICM system and joint detection and decoding.

Alternatively, non-binary LDPC codes $[84,85]$ based on low density parity check matrix where equations are done on a Galois Field of cardinality higher than 2, work directly with constellation symbols which prevent the mutual information loss due to marginalization in the binary case. This performance advantage comes at the expense of higher decoding complexity compared to the binary counterpart. Furthermore, the worldwide efforts in hardware implementation, adoption in practical standards and development of optimal algorithms of non-binary LDPC codes are still far compared to the binary case.

In a MIMO-BICM system, the performance can be further improved by employing iterative MIMO decoding [86]. Here, MIMO demodulator and channel decoder exchange extrinsic (new) information in an iterative fashion. Iterative MIMO-BICM decoding is a key technology to approach the coded-modulation bound. Iterative decoding increases the receiver complexity due to the repeated application of the demodulator and channel decoder blocks. Since iterative decoding only affects the receiver, no modifications are required in standards and transmitters.

Fig. 2.7 presents the conceptual diagram of a iterative decoding process. The soft-in soft-out demodulator computes a-posteriori information about the transmitted code bits in the form of LLRs with the received distorted signal, channel estimates, noise power and in the light of the a-priori information coming from the channel decoder. At the output of the soft-in soft-out detector extrinsic (new) information is computed by subtracting the a-priori LLRs from the a-posteriori information estimates. Exchange of only new information is crucial for the convergence of the iterative process. Then, the extrinsic LLRs are bit-wise de-interleaved to become a-priori information input to the LDPC channel decoder which performs belief propagation iterative message passing algorithm to compute corrected a-posteriori LLRs. Here, extrinsic information is again computed by extracting the a-priori information at the input of the 


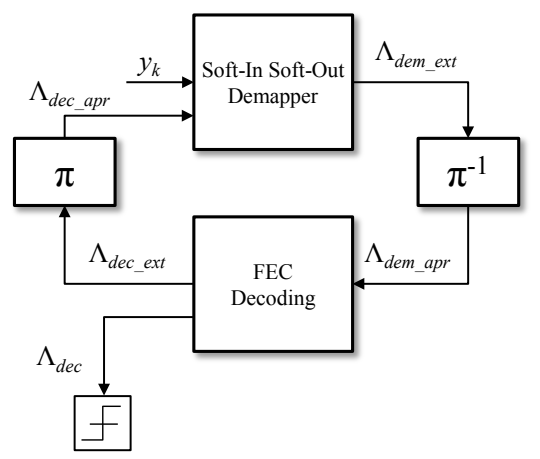

Hard decisions

Figure 2.7: Iterative demapping and decoding block diagram.

channel decoder, and bit interleaved to become a-priori information to the softin soft-out demodulator closing the iterative process loop. Iterative exchange of extrinsic information is performed until a maximum number of iterations is reached or the parity checks are satisfied in which case the channel decoder outputs hard decisions.

Note that at the first iteration no a-priori information is available at the input of the soft-in soft-out demodulator. Hence, an all-zero vector is fed to the demodulator which computes soft reliable information about the transmitted code bits to be fed to the channel decoder. The BICM performance at the first iteration of the iterative decoding process corresponds in fact to the performance of conventional BICM receivers without iterative decoding.

The next subsections describe different algorithms for soft-in soft-out MIMO demodulation from non-linear demodulators with (near) optimal performance but with high computation complexity requirements, to linear demodulators with low computational complexity requirements.

Soft-In Soft-Out MAP demodulator The optimum demodulator in MIMO systems maximizes the a-posteriori probabilities (MAP) of transmitted code bits and minimizes the probability of error. It can be expressed in the form of LLRs by the expression:

$$
\Lambda_{l} \triangleq \log \frac{p\left(c_{l}=1 \mid \mathbf{y}, \mathbf{H}\right)}{p\left(c_{l}=0 \mid \mathbf{y}, \mathbf{H}\right)}=\log \frac{\sum_{\mathbf{x} \in \mathcal{X}_{l}^{1}} p(\mathbf{y} \mid \mathbf{x}, \mathbf{H}) \mathrm{P}\{\mathbf{x}=\mathbf{s}\}}{\sum_{\mathbf{x} \in \mathcal{X}_{l}^{0}} p(\mathbf{y} \mid \mathbf{x}, \mathbf{H}) \mathrm{P}\{\mathbf{x}=\mathbf{s}\}},
$$


where $p\left(c_{l} \mid \mathbf{y}, \mathbf{H}\right)$ is the probability mass function of the transmitted coded bits conditioned to the received vector $\mathbf{y}$ and the channel matrix $\mathbf{H} . \mathcal{X}_{l}^{1}$ and $\mathcal{X}_{l}^{0}$ are the set constellation symbols with the corresponding bit label $c_{l}$ to 1 and 0 , respectively, and the conditional Probability Density Function (pdf) $p(\mathbf{y} \mid \mathbf{x}, \mathbf{H})$ is given by the expression:

$$
p(\mathbf{y} \mid \mathbf{x}, \mathbf{H})=\frac{1}{\left(\pi \sigma^{2}\right)^{N_{r}}} \exp \left(-\frac{\|\mathbf{y}-\mathbf{H} \mathbf{x}\|^{2}}{\sigma^{2}}\right) .
$$

Additionally, $\mathrm{P}\{\mathbf{x}=\mathbf{s}\}$ is the transmitted vector probability that can be further developed as

$$
\mathrm{P}\{\mathbf{x}=\mathbf{s}\}=\prod_{l=1}^{m} \mathrm{P}\left\{c_{l}=b_{l}\right\}=\prod_{l=1}^{m} \frac{\exp \left(-b_{l} L_{a}\left(c_{l}\right)\right)}{1+\exp \left(-L_{a}\left(c_{l}\right)\right)},
$$

where $b_{l} \in\{0,1\}$ is the bit label of $\mathbf{s}$ and $L_{a}\left(c_{l}\right)$ is the a priori LLR for $c_{l}$ which is provided by the LDPC decoder. In the case of non-iterative detection a priori information is not fed back to demodulate the MIMO signal and $L_{a}$ is a zero vector.

Using (2.41) and (2.42) and some expression manipulations [45], (2.41) can be expressed as:

$$
\Lambda_{l}=\log \frac{\sum_{\mathbf{x} \in \mathcal{X}_{l}^{1}} \exp \left(-\frac{1}{\sigma^{2}}\|\mathbf{y}-\mathbf{H} \mathbf{x}\|^{2}+\sum_{j} b_{j} L_{a}\left(c_{j}\right)\right)}{\sum_{\mathbf{x} \in \mathcal{X}_{l}^{0}} \exp \left(-\frac{1}{\sigma^{2}}\|\mathbf{y}-\mathbf{H} \mathbf{x}\|^{2}+\sum_{j} b_{j} L_{a}\left(c_{j}\right)\right)},
$$

where the summation of the a-priori LLRs $L_{a}$ spans all the label bits that equal 1. Despite the optimal error rate performance of MAP demodulator, for each demodulator execution the complexity order is $\mathcal{O}\left(|\mathcal{A}|^{N_{t}}\right)$ where $|\mathcal{A}|$ is the cardinality of the symbol constellation $\mathcal{A}$ (e.g., $|\mathcal{A}|=4$ for QPSK) and $N_{t}$ is the number of transmit antennas. That is, complexity increases exponentially with the number of transmit antennas and linearly with the number of demodulation executions. The complexity of the MAP demodulator is commonly considered as too computationally demanding for most of the practical applications therefore a vast number of suboptimal demodulator with lower complexity have been proposed in the literature.

Soft-In Soft-Out Max-log demodulator To reduce the computational complexity of MAP detection the max-log approximation

$$
\log \left(\sum_{i} \exp \left(x_{i}\right)\right) \approx \max _{i}\left\{x_{i}\right\}
$$


replaces the computation of logarithmic and exponential functions to a minimum distance problem more suitable for hardware implementation with reduced performance loss. Applying the max-log approximation to (2.41) and further manipulations similar to those in (2.44), the computation of the approximate LLRs has the form [45]

$$
\begin{array}{r}
\tilde{\Lambda}_{l}=\min _{\mathbf{x} \in \mathcal{X}_{l}^{0}}\left(\frac{1}{\sigma^{2}}\|\mathbf{y}-\mathbf{H} \mathbf{x}\|^{2}+\sum_{j} b_{j} L_{a}\left(c_{j}\right)\right)- \\
\min _{\mathbf{x} \in \mathcal{X}_{l}^{1}}\left(\frac{1}{\sigma^{2}}\|\mathbf{y}-\mathbf{H} \mathbf{x}\|^{2}+\sum_{j} b_{j} L_{a}\left(c_{j}\right)\right),
\end{array}
$$

where the summation of the a-priori LLRs $L_{a}$ spans all the label bits that equal 1. The complexity for each execution of max-log demodulation has order $\mathcal{O}\left(|\mathcal{A}|^{N_{t}}\right)$, hence still scales exponentially with the number of transmit antennas and linearly with the number of demodulation executions.

Soft-In Soft-Out MMSE demodulator Linear demodulators like MMSE are other kind of efficient receivers with very low computational complexity. They apply a linear equalizer to suppress the stream interference to provide an estimate of the transmitted symbols followed by a per-layer detection. Therefore, the joint MIMO detection problem is transformed into independent single transmit antenna (i.e., one layer) detection problems. The estimates computed by the MMSE equalizer can be improved using the information provided by the channel decoder. This approach was initially proposed for soft MMSE equalization for communication over intersymbol interference (ISI) channels (cf. [87, 88]) and later to wireless communication systems [43, 89, 44]. The linear MMSE receiver for non-iterative schemes is given by [90]:

$$
\tilde{\mathbf{x}}=\left(\mathbf{H}^{\dagger} \mathbf{H}+\sigma^{2} \mathbf{I}\right)^{-1} \mathbf{H}^{\dagger} \mathbf{y}
$$

where $\mathbf{y}=\mathbf{H x}+\mathbf{w}$ is the noisy receive vector, $\mathbf{H}$ is the MIMO channel matrix, $\mathbf{x}$ is the transmit vector. The estimated transmit vector is denoted by $\tilde{\mathbf{x}}$ and $\mathbf{I}$ denotes the identity matrix.

The expression in (2.47) can be generalized to take a-priori information into account. The modified estimate is given by

$$
\hat{\mathbf{x}}=\mu_{\mathbf{x}}+\mathbf{C}_{\mathbf{x}, \mathbf{y}} \mathbf{C}_{\mathbf{y}}^{-1}\left(\mathbf{y}-\mu_{\mathbf{y}}\right)
$$

with $(\mathrm{E}\{\cdot\}$ denotes the expectation operator) 


$$
\begin{aligned}
\mu_{\mathbf{x}} & =\mathrm{E}\{\mathbf{x}\}, \quad \mu_{\mathbf{y}}=\mathrm{E}\{\mathbf{y}\}=\mathbf{H} \mu_{\mathbf{x}}, \\
\mathbf{C}_{\mathbf{x}, \mathbf{y}} & =\mathrm{E}\left\{\mathbf{x} \mathbf{y}^{\dagger}\right\}=\mathbf{C}_{\mathbf{x}} \mathbf{H}^{\dagger}, \\
\mathbf{C}_{\mathbf{y}} & =\mathrm{E}\left\{\mathbf{y} \mathbf{y}^{\dagger}\right\}=\mathbf{H C}_{\mathbf{x}} \mathbf{H}^{\dagger}+\sigma^{2} \mathbf{I} .
\end{aligned}
$$

Using the expressions in (2.49), the estimate in (2.48) can be expressed in terms of $\mu_{\mathbf{x}}$ and $\mathbf{C}_{\mathbf{x}}$, whose elements are given by (note that $\mathbf{C}_{\mathbf{x}}$ is a diagonal matrix)

$$
\begin{aligned}
\mu_{\mathbf{x}_{n}} & =\sum_{\alpha \in \mathcal{A}} \alpha \mathrm{P}\left\{\mathbf{x}_{n}=\alpha\right\}, \quad n=1,2, \ldots, N_{t}, \\
\left(\mathbf{C}_{\mathbf{x}}\right)_{n, n} & =\sum_{\alpha \in \mathcal{A}}|\alpha|^{2} \mathrm{P}\left\{\mathbf{x}_{n}=\alpha\right\}-\left|\mu_{\mathbf{x}_{n}}\right|^{2},
\end{aligned}
$$

where $\mathcal{A}$ is the transmit symbol alphabet in one transmit antenna. Let $|\mathcal{A}|$ denote the cardinality of the symbol alphabet. Then the symbol probabilities in $(2.50)$ can be further developed as

$$
\mathrm{P}\left\{\mathbf{x}_{n}=\alpha\right\}=\prod_{j=1}^{m} \mathrm{P}\left\{c_{n, j}=b_{j}\right\}=\prod_{j=1}^{m} \frac{\exp \left(b_{j} L_{a}\left(c_{n, j}\right)\right)}{1+\exp \left(L_{a}\left(c_{n, j}\right)\right)},
$$

where $b_{j} \in\{0,1\}\left(c_{n, j}\right)$ is the bit label of $\alpha\left(\mathbf{x}_{n}\right)$ and $L_{a}\left(c_{n, j}\right)$ is the a-priori LLR for $c_{n, j}$ which is provided by the LDPC decoder.

For each execution of the soft-in soft-out MMSE-based demodulator, the complexity of such procedure comprises the complexity for the symbol estimation $\mathcal{O}\left(N_{r} N_{t}^{2}\right)$ operations plus the complexity to detect all code bits $\mathcal{O}\left(N_{r} N_{t}|\mathcal{A}|\right)$, where $N_{r}$ is the number of receive antennas. As we observe the complexity for the detection of the independent layers is linear with the number of transmit antennas and linearly with the number of demodulation executions. Hence, a significant complexity reduction can be achieved compared to optimal demodulation.

Fig. 2.8 presents performance results with iterative decoding for two types of soft-in soft-out demodulators, the max-log and MMSE demodulators in previous subsections. This figure shows the FER vs. CNR for code rates $1 / 3$, $8 / 15$, and 11/15 using the MIMO profile of DVB-NGH with eSM-PH. For the max-log demapper, the iterative decoding gain increases with increasing code rate, i.e., $1 \mathrm{~dB}(1 / 3), 1.1 \mathrm{~dB}(8 / 15)$, and $1.8 \mathrm{~dB}(11 / 15)$. Furthermore, the results with MMSE demodulator show that it is able to exploit the benefits of iterative decoding while keeping computational complexity low [89]. In this case, MMSE demodulator gains approximately $0.7 \mathrm{~dB}(1 / 3), 0.9 \mathrm{~dB}(8 / 15)$, and 


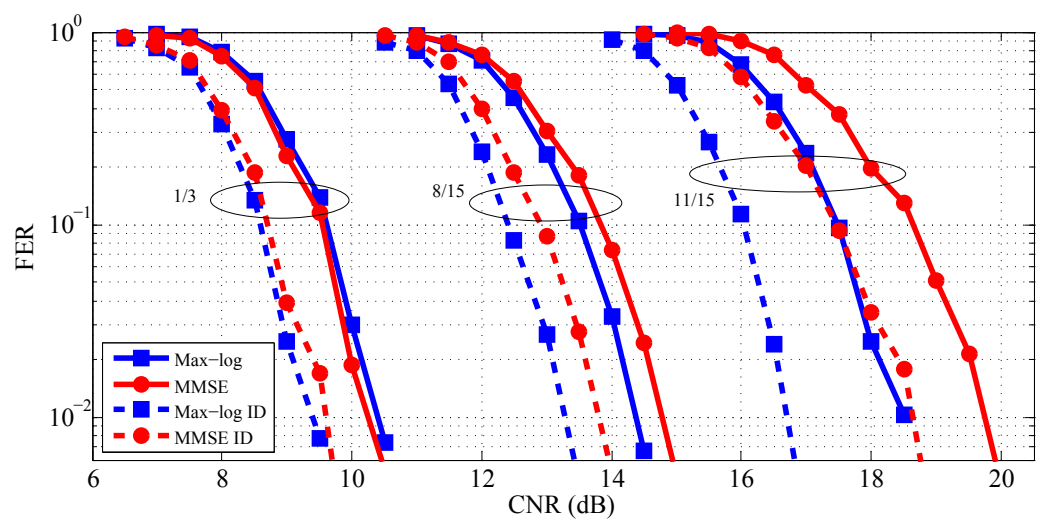

Figure 2.8: FER vs. CNR of iterative decoding with 8 bpc and code rates $1 / 3$, $8 / 15$, and 11/15 in the NGH outdoor MIMO channel with $60 \mathrm{~km} / \mathrm{h}$ speed.

$1.1 \mathrm{~dB}(11 / 15)$ by iterative decoding. However, we observe performance degradation of MMSE demapper compared with the max-log demodulator with the increasing rate. In particular, the max-log demapper outperforms the MMSE demapper by about $-0.15 \mathrm{~dB}(0.2 \mathrm{~dB}), 0.4 \mathrm{~dB}(0.5 \mathrm{~dB})$, and $1.2 \mathrm{~dB}(1.9 \mathrm{~dB})$ for (non-)iterative decoding. It is interesting to note that for all simulated code rates, the MMSE receiver with iterative decoding does not perform worse than the non-iterative max-log receiver.

Other non-linear demodulators Other non-linear solutions like spheredecoding reduce the complexity by finding the most likely transmitted symbol from a subset of the original $|\mathcal{A}|^{N_{t}}$ search. Sphere decoding is based on QR factorization to transform the detection problem into a tree search detection problem. Sphere decoding algorithms can be mainly categorized in two groups if the goal is to target performance of fixed complexity. The former achieves ML solution with complexity dependent on the channel realization [41]. The latter while providing fixed complexity, it does not always achieve ML solution [91]. The study of these kind of solutions are out of the scope of this thesis.

\subsubsection{Mutual Information of MIMO-BICM systems}

This subsection starts by giving the mathematical expressions for the CM and BICM maximum achievable rates and correspond to to the blocks presented in 2.2 .

Given a communication system with a transmit signal $\mathbf{x}$ and a received signal $\mathbf{y}$ through a channel $\mathbf{H}$ with the relationship $\mathbf{y}=\mathbf{H x}$, the coded modulation 
maximum achievable rate is given by:

$$
\mathrm{C}_{\mathcal{X}}^{\mathrm{cm}} \triangleq E_{\mathbf{x}, \mathbf{y}, \mathbf{H}}\left[\log _{2} \frac{f(\mathbf{y} \mid \mathbf{x}, \mathbf{H})}{\frac{1}{2^{\mathrm{m}}} \sum_{\mathbf{x}^{\prime} \in \mathcal{X}} f\left(\mathbf{y} \mid \mathbf{x}^{\prime}, \mathbf{H}\right)}\right],
$$

and BICM achievable rate has the following expression assuming ideal infinitelength interleaver:

$$
\mathrm{C}_{\mathcal{X}}^{\text {bicm }} \triangleq \sum_{j=1}^{m} E_{\mathbf{x}, \mathbf{y}, \mathbf{H}}\left[\log _{2} \frac{\sum_{\mathbf{x}^{\prime} \in \mathcal{X}_{j}^{c_{j}}} f\left(\mathbf{y} \mid \mathbf{x}^{\prime}, \mathbf{H}\right)}{\frac{1}{2} \sum_{\mathbf{x}^{\prime} \in \mathcal{X}} f\left(\mathbf{y} \mid \mathbf{x}^{\prime}, \mathbf{H}\right)}\right],
$$

where the conditional pdf $f(\mathbf{y} \mid \mathbf{x}, \mathbf{H})$ is given by $(2.42), \mathcal{X}$ is the set of possible (constellation) signals of the transmit vector $\mathbf{x}$, the expectation operator $E$ is over all possible transmit, receive and channel signals $\mathbf{x}, \mathbf{y}, \mathbf{H}$, and $m$ is the number of raw bits per channel use (bpc). For (2.52) the term $\mathcal{X}_{j}^{c_{j}}$ denotes the set of transmit vectors which have the $j^{\text {th }}$ bit in the label of $\mathbf{x}$ the value $c_{j} \in[0,1]$.

Expressions (2.51) and (2.52) are the maximum achievable rates for a CM and BICM communication systems with vanishing error probability and with the optimal MAP demodulator which produces a full search among all possible transmit constellation signals $\mathcal{X}$.

It is interesting to present the following inequality [92]:

$$
\mathrm{C}_{\mathcal{X}}^{\mathrm{cm}} \geq \mathrm{C}_{\mathcal{X}}^{\mathrm{bicm}}
$$

the CM capacity is the upper-bound to the BICM capacity. The performance of a BICM system can be improved by iterative demapping and in the limiting case, the BICM with iterative demapping can reach the CM bound.

Fig. 2.9 shows the BICM and CM mutual information against CNR for a $2 \times 2$ MIMO system with co-polar antennas, under a i.i.d. Rayleigh channel with no spatial correlation, and for spectral efficiencies of 4,8 and 12 bpc (which corresponds to QPSK, 16QAM and 64QAM in each transmit antenna, respectively). It can be observed the that CM is always superior than BICM. Additionally, as the CNR increases each curve saturates to the value $m$ which it is recalled as the total number of bpc. For the BICM system, it is interesting to point that the constellation with highest spectral efficiency depends of the operating CNR. At low CNR regime, low order constellations provide higher spectral efficiency that constellations with higher cardinality. This can be observed in the right part of Fig. 2.9, where QPSK is superior to 16QAM and 64QAM up to 7 $\mathrm{dB}$ when 16QAM gives the highest spectral efficiency. However, this behaviour 

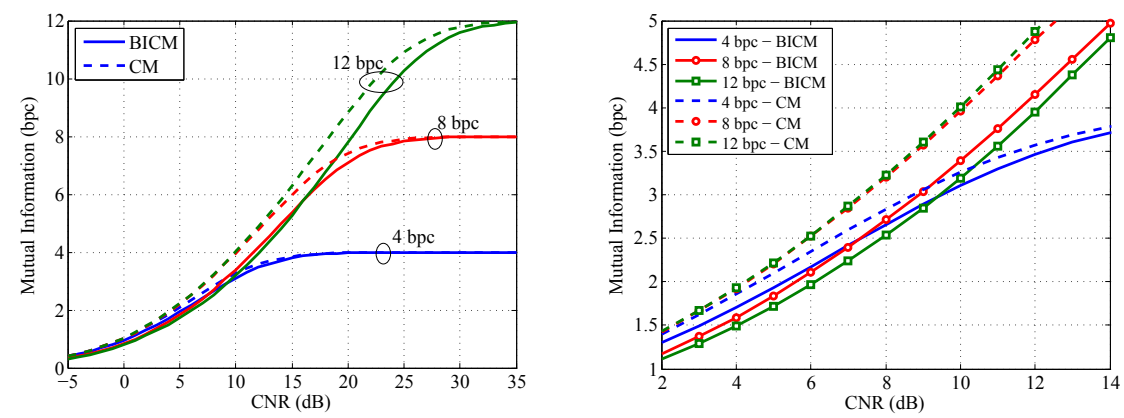

Figure 2.9: Mutual information (bpc) of BICM and CM systems vs. CNR $(\mathrm{dB})$ of a $2 \times 2$ MIMO system with co-polar antennas under a i.i.d. Rayleigh channel with no spatial correlation and no power imbalance and three spectral efficiencies: 4, 8 and $12 \mathrm{bpc}$. The left figure shows the performance in the CNR between -5 to $35 \mathrm{~dB}$ while the right plot shows a zoomed version in a CNR between 2 to $14 \mathrm{~dB}$.

does not occur with CM, where low order constellation always incur into lower or equal spectral efficiency than higher order constellation cases.

\subsection{Quantization}

During the standardization of new communication systems the initial design accounts for perfect reception conditions, e.g., optimal demodulators, perfect CSI, perfect noise power estimation, and infinite-precision number representations. For instance, that was the approach to design digital terrestrial TV broadcasting technologies such DVB-T2, DVB-NGH and ATSC 3.0. However, due to complexity constraints and finite-precision arithmetic, it is crucial for the overall system performance to carefully design receiver algorithms.

So far, receiver hardware design has taken into account finite-precision number representations only in a straightforward manner via simulations, completely neglecting non-uniform quantizers adjusted to the signal properties. In addition, since on-chip memory accounts for a large fraction of the chip area, it is desirable to have small word length with reduced performance loss. Hence, in this dissertation the focus will be put in low-rate quantizers which are suitable for communication systems with constrains in power consumption, memory size and chip-area. 


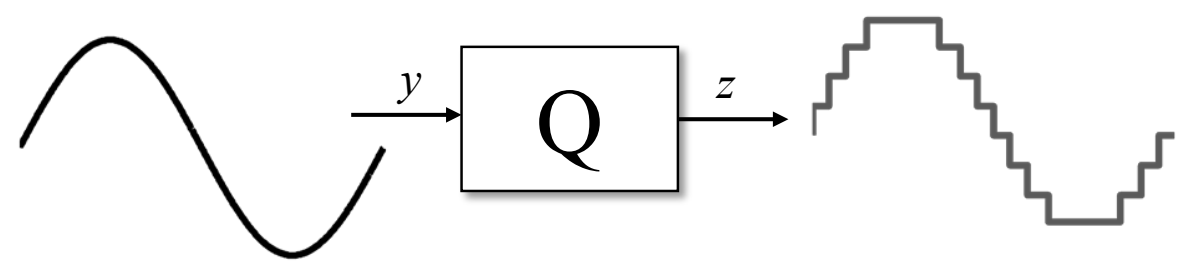

Figure 2.10: Scalar quantization.

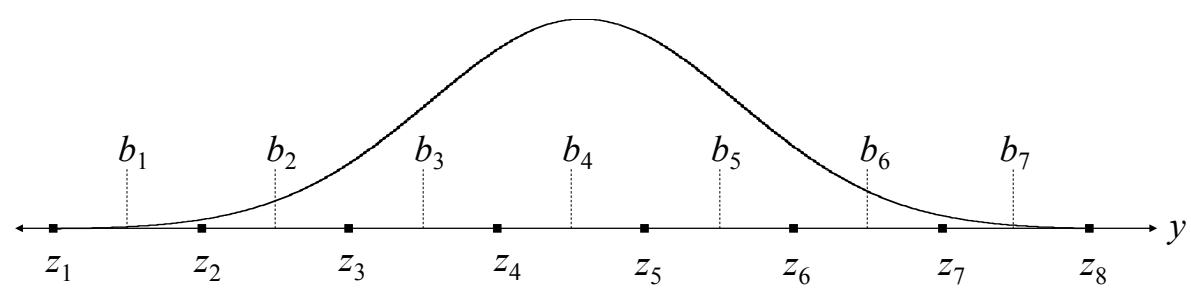

Figure 2.11: Example of quantization boundaries and reproducers.

A $q$ bits scalar ${ }^{2}$ quantizer $Q: \mathbb{R} \mapsto \mathcal{Z}$ maps the input signal $y$ to the quantizer output $z=Q(y)$ where $|\mathcal{Z}|=2^{q}$ (see Fig. 2.10). The quantizer $Q$ is defined by a set of $n$ quantizer reproducers $\left\{z_{1}, \ldots, z_{n}\right\}$ and a set of quantizer boundaries $\left\{b_{0}, \ldots, b_{n}\right\}$, where $\log _{2} n=q$ (see Fig. 2.11). The quantization boundaries partitions the set of $\mathbb{R}$ into $n$ regions $\mathcal{Y}_{i}=\left[b_{i-1}, b_{i}\right), i=1 \ldots, n$, where the first and last boundaries are set to $b_{0}=-\infty$ and $b_{n}=+\infty$. An input value $y \in \mathbb{R}$ to the quantizer is assigned to the reproducer value $z_{i}$ if $y \in \mathcal{Y}_{i}$.

The quantizer reproducers and boundaries are designed as to represent in the most efficient manner the input random variable $y$. This optimization can be carried under different criteria such as minimizing the Mean Squared Error (MSE) or to maximize the mutual information between the data and the output of the quantizer. Quantization algorithms for a MIMO-BICM with iterative decoding receiver will be described in Chapter 4 .

A straightforward approach is to uniformly distribute the quantization boundaries and reproducers. More sophisticated quantizer designs lead to non-uniform distribution of the quantizer reproducers and boundaries by exploiting the signal statistical structure, such as the pdf of the input $p(y)$. However, even with

\footnotetext{
${ }^{2}$ Vector quantization $Q: \mathcal{Y}^{k} \mapsto \mathcal{Z}$, maps a vector signal y of dimension $k$ to quantizer output $z=Q(\mathbf{y})$. Vector quantization in communications systems are proposed in [93, 94, 95]. In this dissertation scalar quantization is considered and vector quantization is out of the scope of this thesis.
} 


\subsection{Quantization}
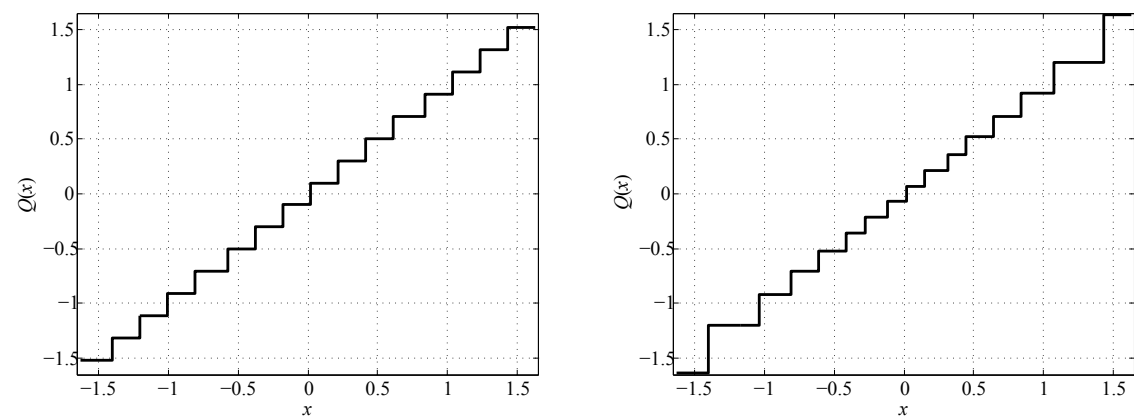

Figure 2.12: Examples of uniform and non-uniform scalar quantization.

the most simple approach of uniform quantization, some kind of information about the input signal is required to select reproducers $z_{1}$ and $z_{n}$ that represent the entire input signal dynamic range. An example of input-output relationship for uniform and non-uniform quantization is illustrated in Fig. 2.12 for an input $y$ Gaussian distributed (cf. Fig. 2.11). We can observe a more dense sampling in the centre part of the graph where the distribution has higher density. 


\section{Chapter 3}

\section{MIMO Precoding for Terrestrial Broadcast Systems}

7 HIS CHAPTER studies the performance and structure of MIMO precoders 1 based on rotation matrices for $2 \times 2$ MIMO and focus on the case of crosspolar antennas, which is the preferred configuration in DTT systems in the UHF band. Analysis and evaluation with the information-theoretic limits of BICM systems and bit-error-rate simulations including channel coding show the interesting results that the performance of the precoder depends on the selected code-rate. Then, the $2 \times 2$ MIMO precoders adopted in DVB-NGH and ATSC 3.0 are analysed and the selected parameters are compared with the results obtained in this chapter.

Furthermore, a channel-precoder is proposed that exploits statistical information of the MIMO channel. The performance of the channel-precoder is evaluated in a wide set of channel scenarios and mismatched channel conditions, a typical situation in the broadcast set-up. Capacity results show performance improvements in the case of strong line-of-sight scenarios with correlated antenna components and resilience against mismatched condition for $4 \times 2 \mathrm{MIMO}$ set-up. Finally, bit-error-rate simulation results compare the performance of single-input single-output, $2 \times 2$ and $4 \times 2$ MIMO systems and the proposed MIMO channel-precoder. 


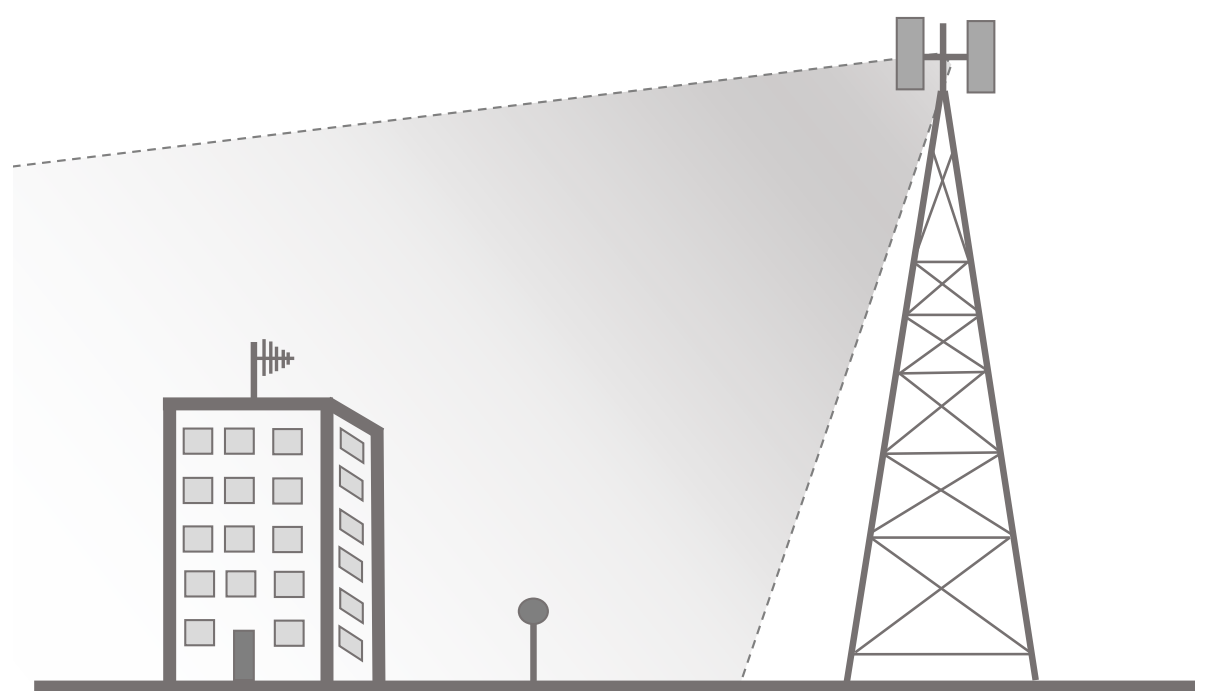

Figure 3.1: Chapter 3 covers the scenario where a single transmitter serves receivers at rooftop reception condition and/or portable/mobile terminals at ground level.

\subsection{Introduction and Scope}

This chapter studies MIMO precoding techniques for receivers with two receive aerials served by a single transmitter site with two or four transmit aerials. This is exemplified in Fig. 3.1. This can model a broadcast network in Multi Frequency Network (MFN) configuration, or a SFN configuration where the signals at the receive antennas from distant transmitter sites have suffered enough attenuation that its effect at the receivers can be discarded. Hence, no distributed signal processing is realized across transmitters in the SFN network. The MIMO precoding techniques studied and proposed in this chapter can be also utilized at each of the transmitter sites in a SFN network. However, the evaluation in such type of networks is out of the scope of this thesis. The multi-antenna receivers can be portable or mobile terminals at ground level, or the antenna can be placed in a rooftop for fixed reception. The channel models utilized in this chapter are presented in Appendix A.

\section{Precoder Position at the Transmitter}

A MIMO precoder applies a linear transformation to the constellation symbols of each of the transmit data streams to enhance the system performance 


\subsection{Introduction and Scope}

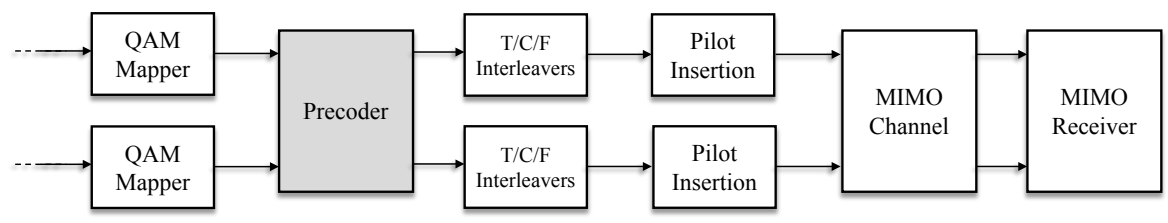

(a) Precoder before Interleaving blocks and pilot insertion

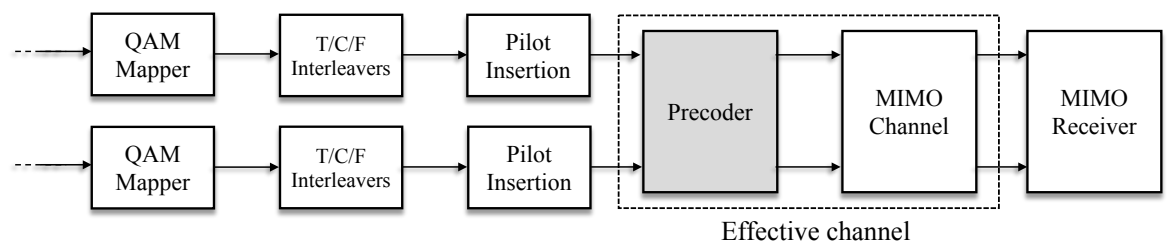

(b) Precoder after Interleaving blocks and pilot insertion

Figure 3.2: Precoding position alternatives in a MIMO transmitter.

(cf. Chapter 2 Section 2.3.3). The position of the MIMO precoder at the transmitter chain can have different implications at the receiver end. Fig. 3.2 presents two alternatives for the MIMO precoding position in a generic MIMO transmitter set-up.

The first option is to place the precoder just after the mapper from bits to constellation symbols and before the subsequent interleaving stages [see Fig. 3.2(a)]. The MIMO profiles of DVB-NGH and ATSC 3.0 follow this architecture. The precoder could also be placed after the interleaving stages and prior to the pilot insertion block without affecting the output of the final transmitted signal. This is due to the fact that the different interleaving operations and the precoding across the transmit antenna streams are orthogonal processes. Interleaving stages, such as time and frequency interleavers, apply the same permutations at each of the antenna streams and the precoding process the constellation symbols in the space domain. With this architecture the demodulation process at the receiver takes into account the precoding transformation applied at the transmitter. The receiver implications of the demodulation of a MIMO precoded signal at different positions of the chain will be discussed in Chapter 4.

The second alternative of the position of the precoder is shown in Fig. 3.2(b). In this case, the precoder is placed after the pilot insertion which allows transmission potentially transparent to receiver terminals. Since the precoder is placed after the pilots, using the pilot information the receiver estimates the combined effect of the precoder and the channel as an effective channel. Transmission techniques transparent to receiver terminals can provide flexibility to 


\section{CHAPTER 3. MIMO PRECODING FOR TERRESTRIAL} BROADCAST SYSTEMS

network operators for the introduction of new schemes to the existing receiving population in its network. If the precoding transformation varies along the time or frequency domain, it can impose a performance degradation if the resulting channel selectivity cannot be estimated at the receiver. To avoid any potential degradation the precoder can be placed before the pilot insertion [as in Fig. 3.2(a)]. Alternatively, a precoder with no variation in frequency or time domains could be transparent to receivers and without imposing any additional distortion to the channel frequency response.

\subsection{Precoding Based on Rotation Matrices}

The precoding structure based on rotation matrices considered in this section for $2 \times 2$ MIMO systems has the following mathematical expression:

$$
\left[\begin{array}{l}
x_{1} \\
x_{2}
\end{array}\right]=\underbrace{\left[\begin{array}{cc}
\sqrt{\beta} & 0 \\
0 & \sqrt{1-\beta}
\end{array}\right]}_{\text {power imbalance }} \underbrace{\left[\begin{array}{cc}
\cos \theta & \sin \theta \\
\sin \theta & -\cos \theta
\end{array}\right]}_{\text {rotation }} \underbrace{\left[\begin{array}{cc}
\sqrt{\alpha} & 0 \\
0 & \sqrt{1-\alpha}
\end{array}\right]}_{\text {stream power allocation }}\left[\begin{array}{l}
s_{1} \\
s_{2}
\end{array}\right] \text {. }
$$

Three precoding components in (3.1) can be identified: stream power allocation, rotation and Power Imbalance (PI).

Stream power allocation It distributes the transmit power across the information streams $s_{1}$ and $s_{2}$, and it is controlled by the parameter $\alpha \in[0,1]$. This unequal power allocation between data streams can be specially interesting when $s_{1}$ and $s_{2}$ are mapped to constellation sets with different cardinality, e.g., $s_{1}$ and $s_{2}$ are mapped to a QPSK and 16QAM constellations, respectively. This will be investigated in the following subsections.

Power imbalance The PI matrix varies the transmit power allocated at each transmit antenna according to the parameter $\beta \in[0,1]$. Transmit PI between antennas can occur due to intrinsic physical characteristics of the channel, such as different antenna diagrams for vertical and horizontal polarizations and/or asymmetries in the propagation channel. An imbalance between transmit antennas can also be intentionally generated by the network operators to ease the use of dual polar MIMO operation in some networks [14]. In this thesis, the PI matrix is not included as part of the precoder but is included as part of the channel. This provides a more generic approach, since the performance evaluations under different imbalance values cover both, intentional imbalances introduced by network operators and imbalances produced as part of the channel. 


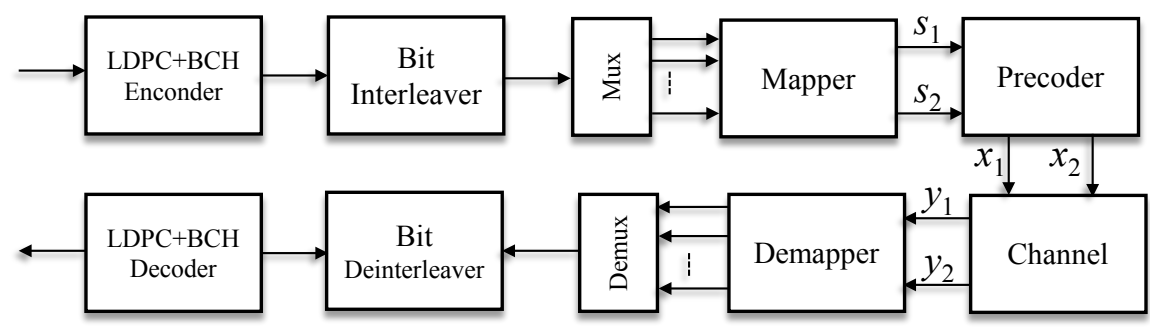

Figure 3.3: Transmit to receive chain for the analysis and evaluations of the $2 \times 2$ MIMO system with precoding based on rotation matrices.

Rotation The rotation matrix linearly combines the information streams according to the rotation parameter $\theta \in[0,2 \pi]$ (cf. Chapter 2, Section 2.3.3). The optimization of the parameter $\theta$ under different transmission parameters and channel conditions will be a core part of this chapter.

The following two subsections characterize the performance of the MIMO precoding based on rotation matrices for different channel conditions such as spatial correlation, transmit PI and antenna orientation (cross-polar vs. copolar), and different transmission parameters such as constellation order and code rate. First, the analysis of the precoder performance is carried using the BICM and CM information theoretic limits. These metrics provide the performance with ideal FEC and bit-interleaving and therefore providing a performance analysis independent of specific implementations. Secondly, the CNR requirements of the MIMO precoder, using a generic physical layer with FEC and bit-interleaving, are investigated. This study will provide with system CNR gains due to precoding.

The block diagram in Fig. 3.3 presents the transmit to receive chain used in the following two subsections and based in Fig. 2.2 of Chapter 2. The main parameters for each of the blocks are detailed next. The channel encoders $(\mathrm{LDPC}+\mathrm{BCH})$ are selected from the ATSC 3.0 specification with a code-word length of 64800 bits with code-rates of $2 / 15,8 / 15$ and $13 / 15$, which are the lowest, mid and highest code-rates available in the specification. The bitinterleaver and bit-deinterleaver use random generated interleaving sequences to make as generic as possible the analysis without the need to rely on specific implementations. The multiplexer and mapper map bits to constellation symbols using QAM constellations and Gray mapping with the following spectral efficiencies: $4 \mathrm{bpc}$ (which correspond to QPSK in each transmit antenna), 6 bpc (which correspond to QPSK and 16QAM in the first and second antenna, respectively), $8 \mathrm{bpc}$ (which correspond to 16QAM in each transmit antenna) and $12 \mathrm{bpc}$ (which correspond to 64QAM in each transmit antenna). These 


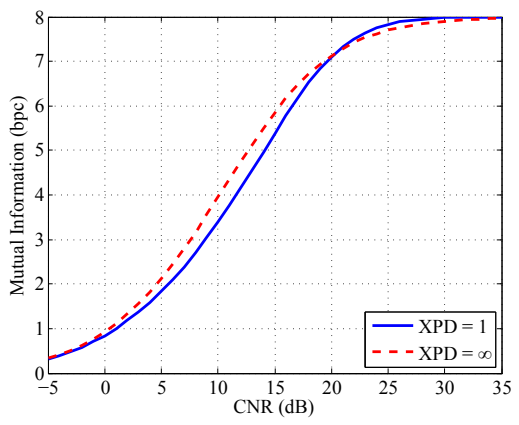

(a) BICM

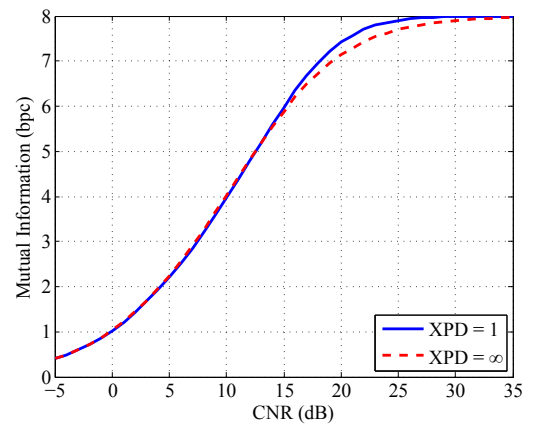

(b) $\mathrm{CM}$

Figure 3.4: Mutual information (bpc) vs. CNR (dB) for MIMO $2 \times 2$ system with $\mathrm{XPD}=1$ (copolar) and $\mathrm{XPD}=\infty$ (cross-polar) with $8 \mathrm{bpc}$ under i.i.d.

Rayleigh channel with no spatial correlation and no power imbalance.

spectral efficiencies are included in DTT standards such as DVB-NGH and ATSC3.0. The channel is i.i.d. Rayleigh MIMO $2 \times 2$ channel (cf. Appendix A section A.1) where the following physical characteristics are included in the analysis: antenna orientation, i.e., cross-polar vs. co-polar, spatial correlation, and PI between transmit antennas, all important parameters of DTT channels. Finally, at the receiver side perfect knowledge of the receive CSI is assumed and an optimal MAP demapper is employed.

\subsubsection{BICM Capacity Analysis}

Figures 3.4 to 3.6 present mutual information in bpc vs. CNR in dB and show the effect of different channel parameters and transmission modes in the system capacity for BICM (left plots) and CM (right plots) systems. In particular, Fig. 3.4 compares the effect of the antenna orientation (co-polar XPD=1 and cross-polar $\mathrm{XPD}=\infty$ ) with 8 bpc. As it can be seen in the BICM system, crosspolar antennas outperform co-polar antennas in the mid CNR range. On the other hand, the co-polar set-up slightly improves the cross-polar performance at high CNRs. Similar tendency can be observed in CM system although here both antenna set-ups show similar performance at low and mid CNRs.

Fig. 3.5 studies the effect of spatial correlation in a co-polar system with 4,8 and $12 \mathrm{bpc}$. In the case of perfect cross-polar antennas (i.e., $\mathrm{XPD}=\infty$ ), the correlation does not significantly impact the channel capacity due to the orthogonal nature of the cross-polar channel. Here, it can be observed, for all spectral efficiencies, a significant performance degradation, although this degradation is higher for BICM than for CM. 


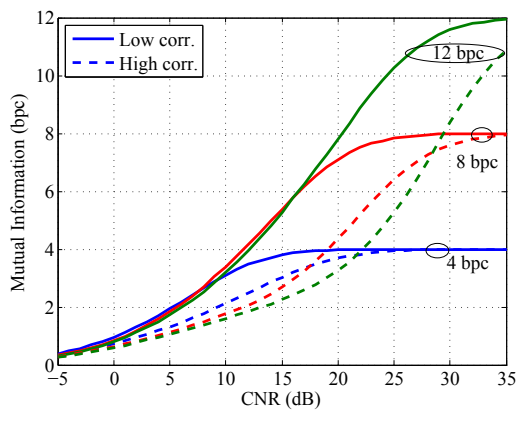

(a) BICM

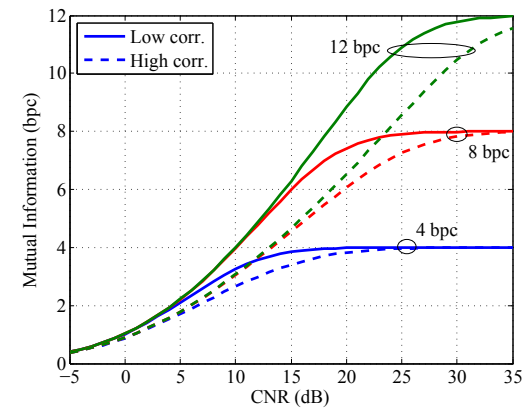

(b) $\mathrm{CM}$

Figure 3.5: Mutual information (bpc) vs. CNR (dB) for MIMO $2 \times 2$ system with XPD $=1$ (copolar) with 4, 8 and 12 bpc under i.i.d. Rayleigh channel with high spatial correlation and no power imbalance.

Fig. 3.6 analyses the effect of PI between transmit antennas with $8 \mathrm{bpc}$, cross-polar antennas and with $P I$ values of $0,3,6$ and $9 \mathrm{~dB}$. For both BICM and CM systems, an increasing degradation is observed with increasing $P I$ values, specially in the mid to high CNR regime.

At this point, the performance of the MIMO precoder is characterized against the previous channel conditions and different precoding parameters. First, the focus will be put on the performance of the rotation matrix for different rotation angles. After, the effect of the stream-power-allocation will be analysed with special attention to the case of 6 bpc.

The figures of mutual information against rotation angle represent three spectral efficiencies corresponding (approximately) to the $m \times r$ value (where $m$ is the number of bpc and $r$ is the FEC code-rate) to allow direct comparison with the results in Section 3.2.2 with FEC and bit-interleaving. As an example, in Fig. 3.7 where $4 \mathrm{bpc}$ is studied, CNR values that provide spectral efficiencies around $0.53,2.13$ and 3.46 are plotted. As for the previous mutual information analysis, the performance of BICM and CM is included. The results with mutual information show that there exists symmetries along the $\theta$ range. The results in this chapter will show sufficient $\theta$ range to observe the symmetries. Furthermore, it was confirmed with simulations that the symmetries are maintained over the entire $\theta \in[0,2 \pi)$ range.

Figures 3.7 to 3.9 analyse the effect of the antenna orientation (co-polar or cross-polar) on the rotation performance for 4,8 and $12 \mathrm{bpc}$, respectively. Here, we can observe in the three figures that, irrespectively of the constellation and spectral efficiency, there is no impact of the rotation in the system performance with co-polar antennas (with no correlation nor PI). This is a reasonable result 


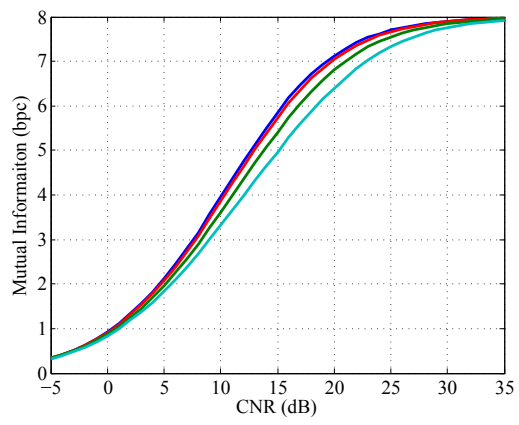

(a) BICM

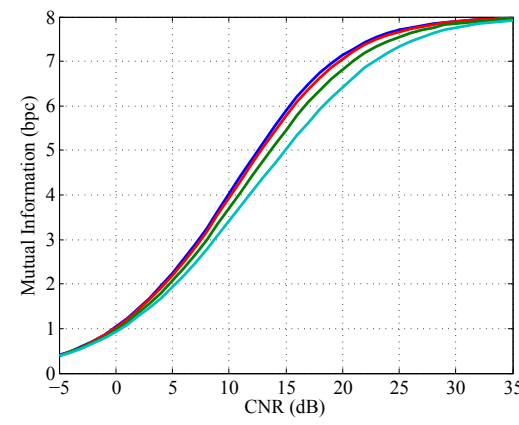

(b) $\mathrm{CM}$

Figure 3.6: Mutual information (bpc) vs. CNR (dB) for MIMO $2 \times 2$ system with $\mathrm{XPD}=\infty$ (cross-polar) with $8 \mathrm{bpc}$ under i.i.d. Rayleigh channel with no spatial correlation and power imbalance between transmit antennas with values of $0,3,6$ and $9 \mathrm{~dB}$ (with increasing performance degradation). BICM (left) and CM (right).

since this channel already mixes up the information streams in a channel with rich scattering. This situation differs for cross-polar antennas, where rotation does affect the system performance. For instance with $4 \mathrm{bpc}$, no rotation is the optimal choice with low code-rate (or equivalently low CNR), but for higher rates, the optimal choice is rotation. In addition, the optimal angle increases with the code-rate, although a minor improvement in spectral efficiency is obtained. Similar tendency is observed for $8 \mathrm{bpc}$ and $12 \mathrm{bpc}$, although at mid code-rate the optimal rotation angle reduces. It is interesting to note that optimal rotation angle with CM system are different to the ones with a BICM system.

Fig. 3.10 summarizes the results of figures 3.7 to 3.9 presenting the BICM system mutual information against CNR without rotation (solid lines) and optimal rotation angle (dashed lines). As it can be seen, at low CNRs both sets of curves overlap, showing that optimal performance is achieved with zero degrees rotation. On the other hand, rotation angles different to zero can enhance the performance at higher CNR values.

Fig 3.11 characterizes the rotation performance in case of co-polar antennas with high spatial correlation. In this case, for all spectral efficiencies, rotation always improves the performance. 


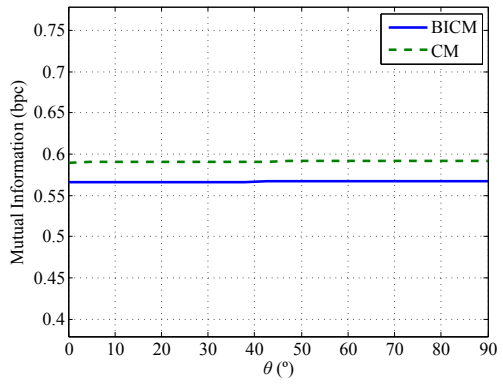

(a) 4 bpc, CR $2 / 15, \mathrm{XPD}=1$

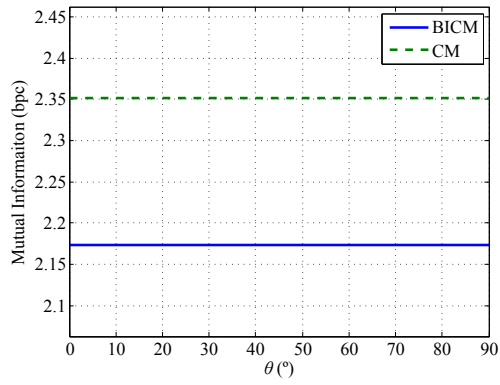

(c) $4 \mathrm{bpc}, \mathrm{CR} 8 / 15, \mathrm{XPD}=1$

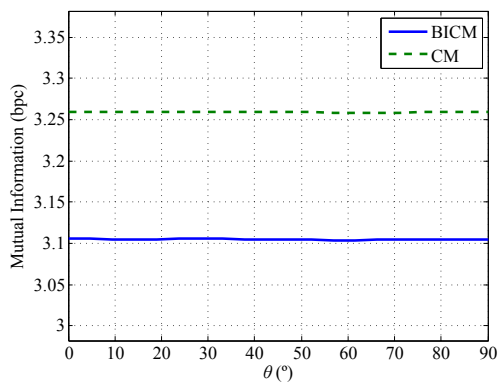

(e) 4 bpc, CR $13 / 15, \mathrm{XPD}=1$

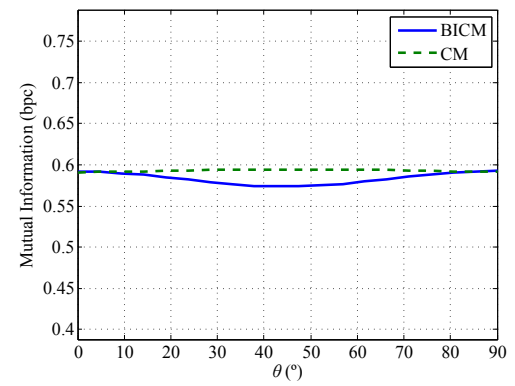

(b) 4 bpc, CR $2 / 15, \mathrm{XPD}=\infty$

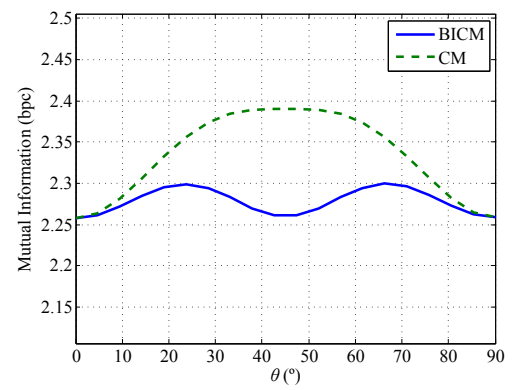

(d) $4 \mathrm{bpc}, \mathrm{CR} 8 / 15, \mathrm{XPD}=\infty$

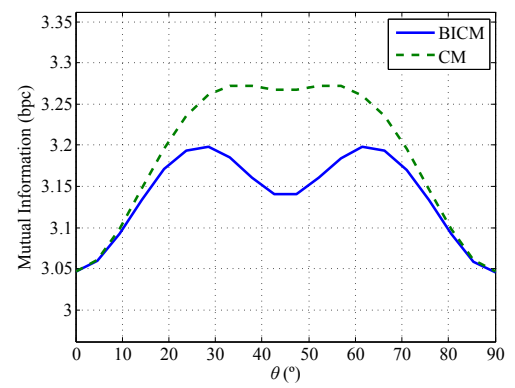

(f) $4 \mathrm{bpc}, \mathrm{CR} 13 / 15, \mathrm{XPD}=\infty$

Figure 3.7: Mutual information (bpc) vs. rotation angle $\theta\left(^{\circ}\right.$ ) for BICM and $\mathrm{CM}$ systems with $4 \mathrm{bpc}$ in a MIMO $2 \times 2$ under i.i.d. Rayleigh channel with no spatial correlation and power imbalance. This figure compares the rotation performance behaviour for $\mathrm{XPD}=1$ (copolar) and $\mathrm{XPD}=\infty$. 


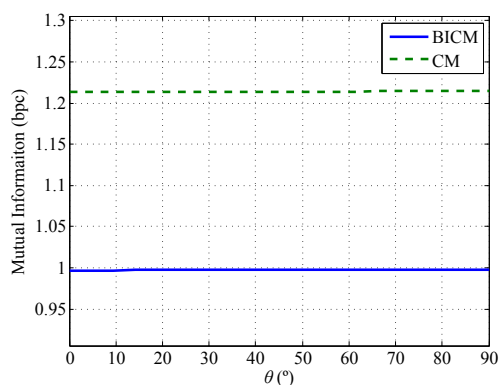

(a) 8 bpc, CR $2 / 15, \mathrm{XPD}=1$

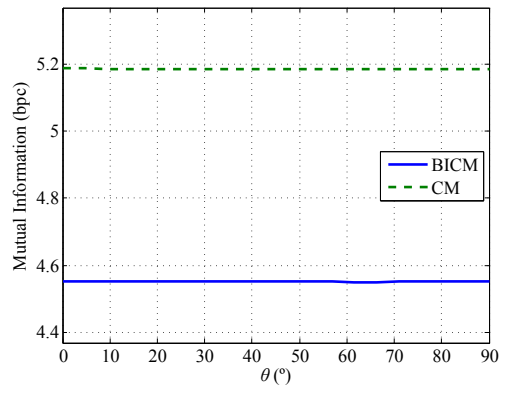

(c) 8 bpc, CR $8 / 15, \mathrm{XPD}=1$

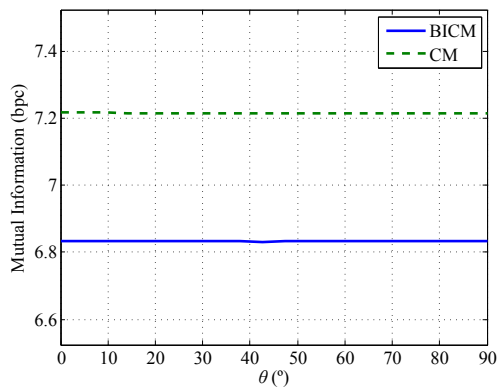

(e) $8 \mathrm{bpc}, \mathrm{CR} 13 / 15, \mathrm{XPD}=1$

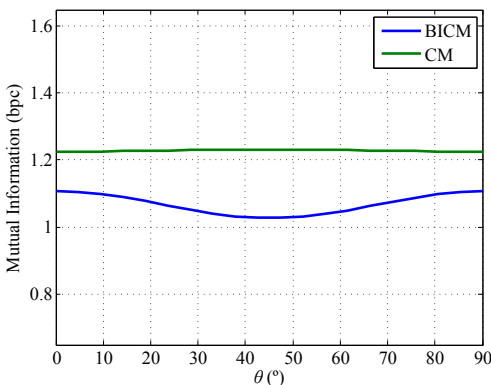

(b) 8 bpc, CR $2 / 15, \mathrm{XPD}=\infty$

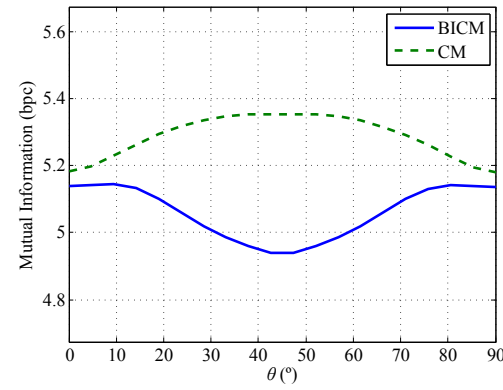

(d) $8 \mathrm{bpc}, \mathrm{CR} 8 / 15, \mathrm{XPD}=\infty$

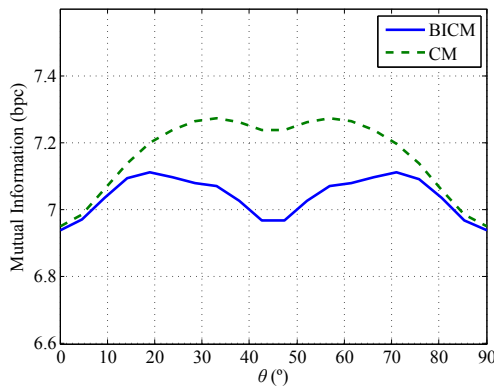

(f) $8 \mathrm{bpc}, \mathrm{CR} 13 / 15, \mathrm{XPD}=\infty$

Figure 3.8: Mutual information (bpc) vs. rotation angle $\theta\left(^{\circ}\right)$ for $\mathrm{BICM}$ and $\mathrm{CM}$ systems with $8 \mathrm{bpc}$ in a MIMO $2 \times 2$ under i.i.d. Rayleigh channel with no spatial correlation and power imbalance. This figure compares the rotation performance behaviour for $\mathrm{XPD}=1$ (copolar) and $\mathrm{XPD}=\infty$. 


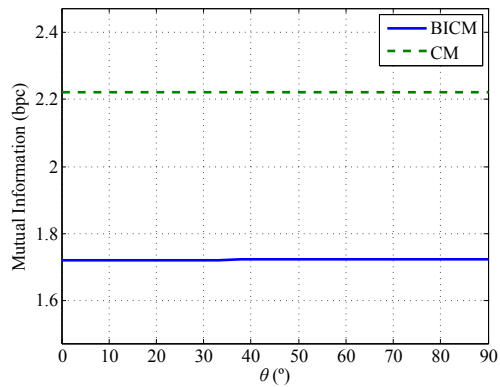

(a) 12 bpc, CR 2/15, XPD=1

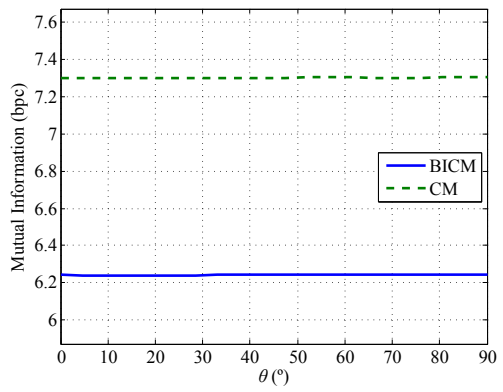

(c) $12 \mathrm{bpc}, \mathrm{CR} 8 / 15, \mathrm{XPD}=1$

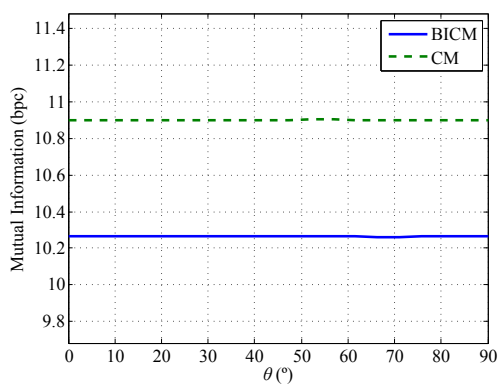

(e) 12 bpc, CR 13/15, XPD=1

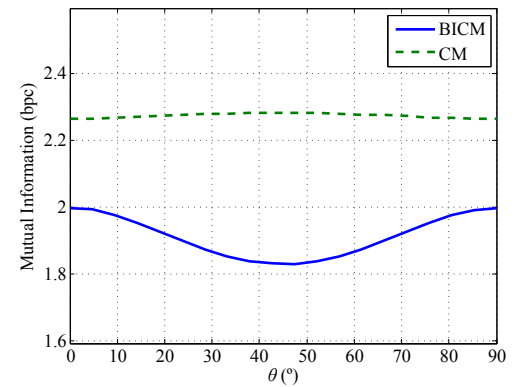

(b) 12 bpc, CR 2/15, XPD $=\infty$

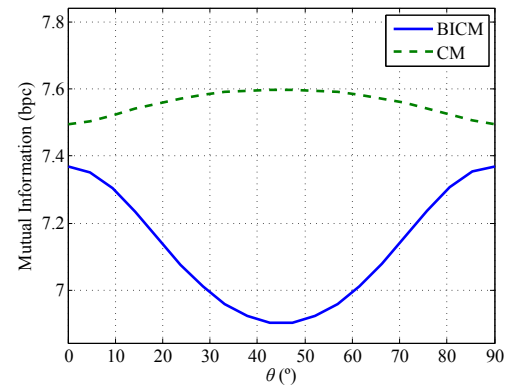

(d) $12 \mathrm{bpc}, \mathrm{CR} 8 / 15, \mathrm{XPD}=\infty$

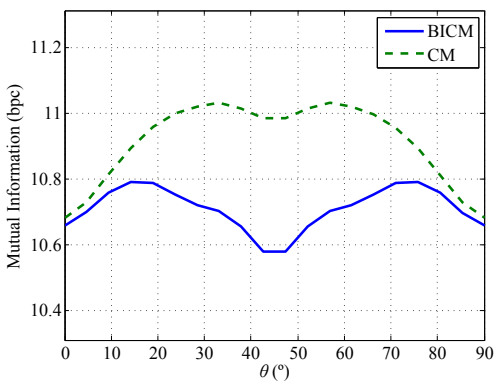

(f) $12 \mathrm{bpc}, \mathrm{CR} 13 / 15, \mathrm{XPD}=\infty$

Figure 3.9: Mutual information (bpc) vs. rotation angle $\theta\left(^{\circ}\right.$ ) for BICM and $\mathrm{CM}$ systems with $12 \mathrm{bpc}$ in a MIMO $2 \times 2$ under i.i.d. Rayleigh channel with no spatial correlation and power imbalance. This figure compares the rotation performance behaviour for $\mathrm{XPD}=1$ (copolar) and $\mathrm{XPD}=\infty$. 


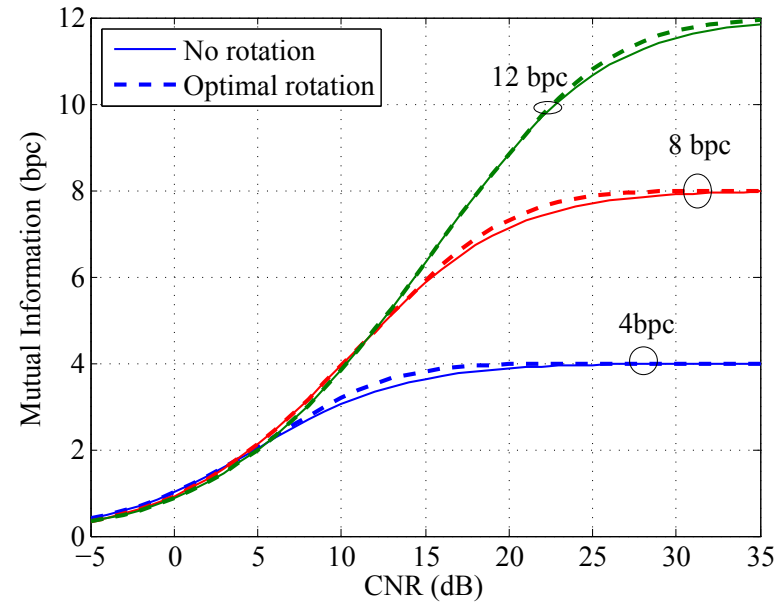

Figure 3.10: Mutual information (bpc) vs. CNR (dB) for MIMO $2 \times 2$ system with $\mathrm{XPD}=\infty$ (cross-polar) with 4, 8 and $12 \mathrm{bpc}$ under i.i.d. Rayleigh channel with no spatial correlation and no power imbalance. Performance without rotation and with optimal rotation angle are presented. 


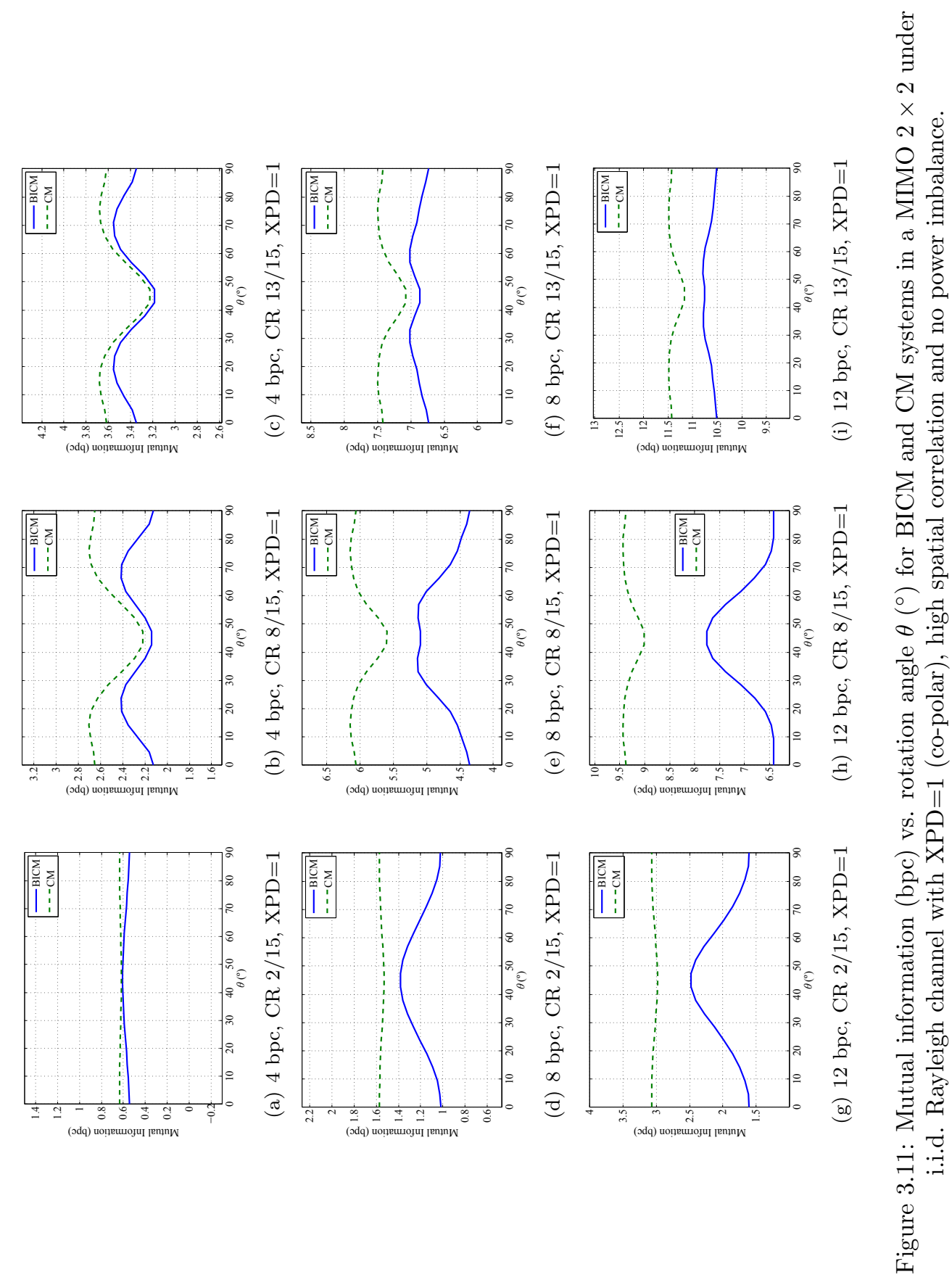




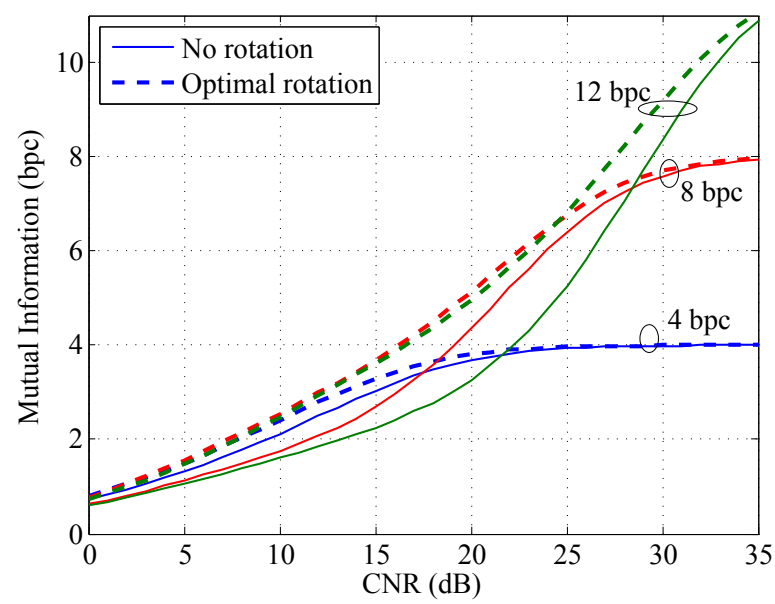

Figure 3.12: Mutual information (bpc) vs. CNR (dB) for MIMO $2 \times 2$ system with $\mathrm{XPD}=1$ (co-polar) with 4,8 and $12 \mathrm{bpc}$ under i.i.d. Rayleigh channel with high spatial correlation and no power imbalance. Performance without rotation and with optimal rotation angle presented.

Fig. 3.12 summarizes the results in Fig. 3.11 presenting the BICM system mutual information against CNR. Here, significant performance improvements are observed specially at 8 and $12 \mathrm{bpc}$.

Now the focus is given to the case with PI between transmit antennas and cross-polar arrangement. Fig. 3.13 shows the rotation performance in the case of $4 \mathrm{bpc}$ (left curves) and $8 \mathrm{bpc}$ (right curves) with $P I$ equal 3,6 and $9 \mathrm{~dB}$ of imbalance. Here, we can see a similar tendency to the results in in Fig. 3.7 and Fig. 3.8, i.e., optimal rotation increases with the code-rate (or system CNR). The summary of the results with $P I=9 \mathrm{~dB}$ in Fig. 3.14 show this conclusion, i.e., zero degrees rotation is optimal at low CNRs, but rotation can improve the performance at higher CNRs. It was confirmed with simulations the same behaviour of the rotation performance with $P I=-9 \mathrm{~dB}$. This is due to the symmetry of the MIMO channel without PI, and the symmetry of the signal processing at each of the transmit streams, i.e., constellations and mapping.

Fig. 3.15 represents the precoding performance with PI between transmit antennas and $6 \mathrm{bpc}$. The PI takes the values from $P I=-9 \mathrm{~dB}$ to $P I=+9 \mathrm{~dB}$ with a $3 \mathrm{~dB}$ step. The left plots illustrate the $P I \geq 0$ (more power allocated to the first transmit antenna) and the right plots illustrate the $P I \leq 0$ (more power allocated to the second transmit antenna). In this scheme with $6 \mathrm{bpc}$, a QPSK constellation is transmitted in the first transmit antenna, while a 16QAM constellation is transmitted in the second transmit antenna. Due to the 


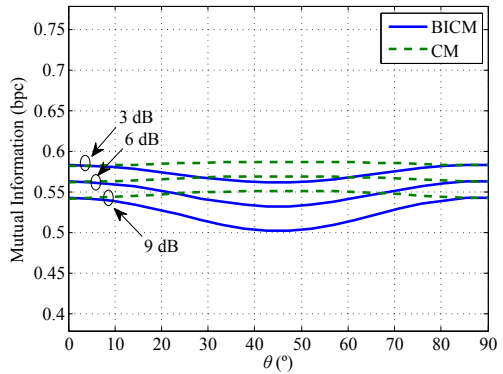

(a) 4 bpc, CR 2/15, XPD $=\infty$

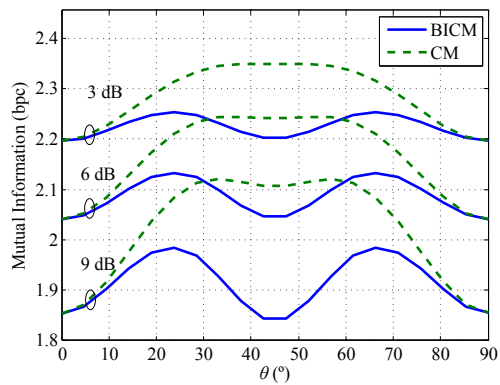

(c) $4 \mathrm{bpc}, \mathrm{CR} 8 / 15, \mathrm{XPD}=\infty$

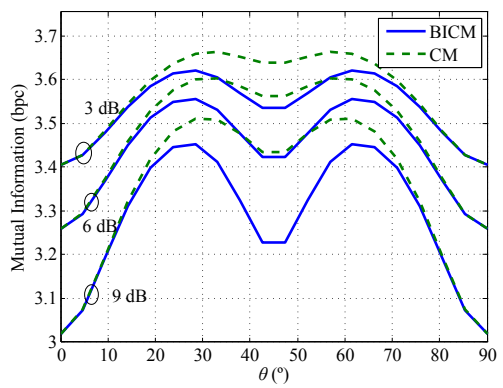

(e) 4 bpc, CR $13 / 15, \mathrm{XPD}=\infty$

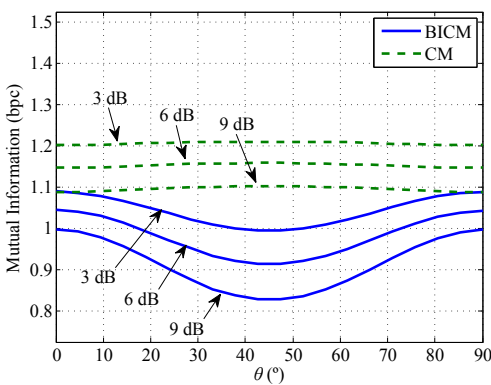

(b) 8 bpc, CR $2 / 15, \mathrm{XPD}=\infty$

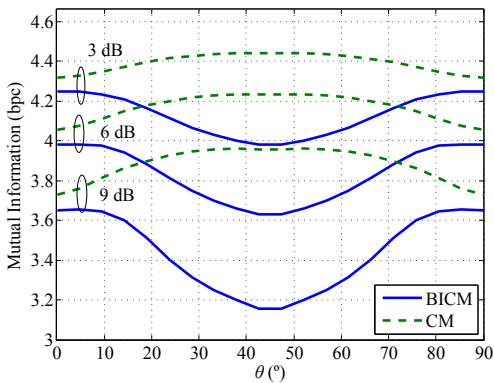

(d) 8 bpc, CR $8 / 15, X P D=\infty$

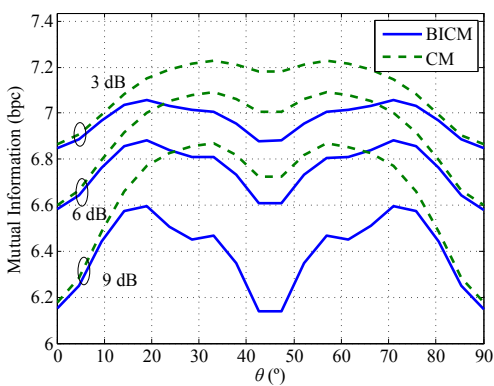

(f) $8 \mathrm{bpc}, \mathrm{CR} 13 / 15, \mathrm{XPD}=\infty$

Figure 3.13: Mutual information (bpc) vs. rotation angle $\theta\left(^{\circ}\right)$ for BICM and

$\mathrm{CM}$ systems in a MIMO $2 \times 2$ with $\mathrm{XPD}=\infty$ under i.i.d. Rayleigh channel with no spatial correlation and power imbalance with values of 3,6 and $9 \mathrm{~dB}$. This figure compares rotation performance behaviour with $4 \mathrm{bpc}$ and $8 \mathrm{bpc}$. 


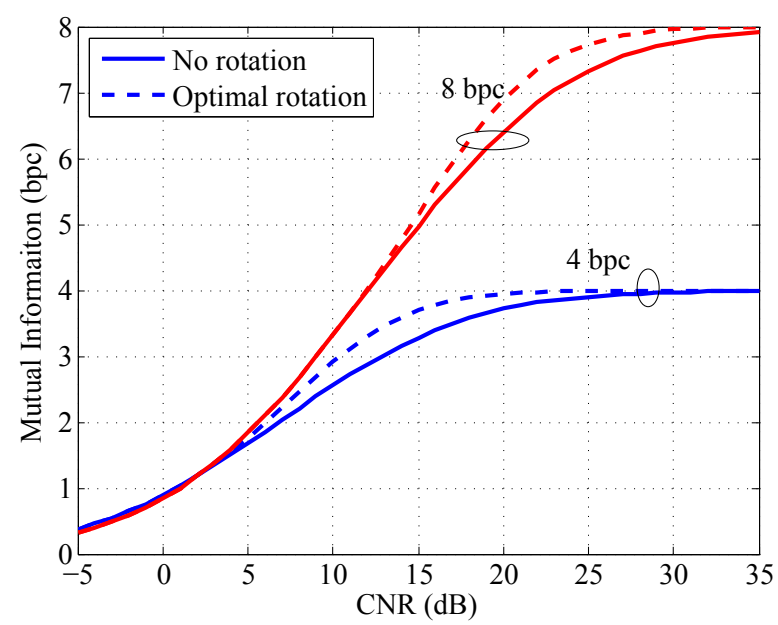

Figure 3.14: Mutual information (bpc) vs. CNR (dB) for MIMO $2 \times 2$ system with $\mathrm{XPD}=\infty$ (cross-polar) with 4 , and 8 bpc under i.i.d. Rayleigh channel

without spatial correlation and power imbalance of $9 \mathrm{~dB}$. Performance without rotation and with optimal rotation angle presented.

different constellation cardinalities, there exists interesting interactions between the rotation and transmit PI that will be presented next. As we can observe in the figures, there is symmetry with respect to the mutual information against the rotation angle. For low code-rate $2 / 15$, the best absolute performance is given by the $P I=0$ case, for both $P I \geq 0$ and $P I \leq 0$. In this case, $0^{\circ}$ is the optimal rotation angle, although the difference with the achieved mutual information at $90^{\circ}$ is marginal. With $P I \geq 0$ (i.e., more power allocated to the first transmit antenna), the optimal rotation angle is $90^{\circ}$, which in effect allocates more power to the 16QAM constellation. This means that at low coderate is optimal to assign more power to the less robust constellation. With $P I \leq 0$ (more power allocated to the second transmit antenna), the optimal rotation angle is $0^{\circ}$, which allocates more power to the 16QAM constellation. For mid code-rate $8 / 15$, it is interesting to note that balancing the transmit power between the antennas by $3 \mathrm{dBs}$ can outperform the $P I=0$ case. With $P I \leq 0$ (more power allocated to the second transmit antenna), the optimal rotation angle is zero degrees, which means that more power is assigned to the constellation with higher cardinality. Additionally, with $P I \geq 0$ (more power assigned to the first antenna), the optimal rotation angle is $90^{\circ}$, which in effect allocates more power to the 16QAM constellation. Finally, for high code-rate $13 / 15$, the results show again the best absolute performance can be 
obtained with $P I=0 \mathrm{~dB}$ combined with rotation. The results also show that with $P I \leq 0$ (more power allocated to the second transmit antenna) and zero degrees of rotation, imbalance values of $P I=-3$ and $P I=-6 \mathrm{~dB}$ improve the $P I=0$ case. Complementary, for the $P I \geq 0$ (more power allocated to the first transmit antenna) this effect appears with $90^{\circ}$.

\subsubsection{System Performance}

To complement the mutual information analysis, the next results characterize the rotation performance with FEC and bit-interleaving. This subsection studies the CNR requirements vs. the rotation angle at a target performance of BER $10^{-4}$ before $\mathrm{BCH}$, which is a sensible value between simulation time and accuracy.

Figures 3.16 to 3.18 compare the performance of co-polar vs. cross-polar without spatial correlation nor PI between transmit antennas for 4, 8 and 12 bpc, respectively. The results are aligned with the mutual information analysis. First, they show that rotation does not reduce the CNR requirements for copolar antennas. For cross-polar antennas, these results demonstrate that at low code-rates the optimal rotation angle is zero degrees, but higher angles optimize the performance with increasing code-rate. The optimal rotation angles are higher for lower order constellations. This can be seen comparing the optimal angles of 4, 8 and $12 \mathrm{bpc}$. The optimal rotation angles and CNR gains are summarized in Table 3.1.

Fig. 3.19 to 3.21 represent the CNR requirements for the case of co-polar antennas and high spatial correlation for 4,8 and $12 \mathrm{bpc}$, respectively. The results are well aligned with the mutual information analysis. Here, rotation provides significant performance improvements (up to $5 \mathrm{dBs}$ for $12 \mathrm{bpc}$ and $2 / 15$ code-rate) in all cases. The optimal rotation angle and CNR gains are presented in Table 3.1.

Fig. 3.22 and Fig. 3.23 present the CNR requirements in the scenario of cross-polar antennas with PI between transmit antennas of 3, 6 and $9 \mathrm{dBs}$, for 4 and 8 bpc. Here, a good alignment with the mutual information analysis can be seen. As in the other analysis without PI and cross-polar antennas, zero degress is the optimal choice at low code-rate, but higher rotation angles optimize the system performance at increasing code-rate. These gains can be significant and up to $4 \mathrm{dBs}$ in the case of $P I=9 \mathrm{~dB}$ and $4 \mathrm{bpc}$ with $13 / 15$ code-rate. Optimal rotation angles and CNR gains are presented in Table 3.1.

Fig. 3.24 shows the CNR requirements in the scenario of cross-polar antennas with PI between transmit antennas from $P I=-9$ to $P I=+9 \mathrm{dBs}$. In this case $6 \mathrm{bpc}$ is evaluated. First, BER results presented are in agreement with the mutual information results presented in Fig. 3.15. At low code-rate 2/15, the best performance is obtained with $P I=0$ regardless of the $\theta$ value. For this 


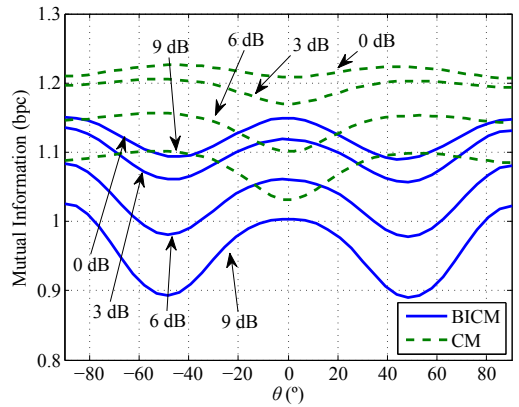

(a) 6 bpc, CR $2 / 15, \mathrm{PI} \geq 0$

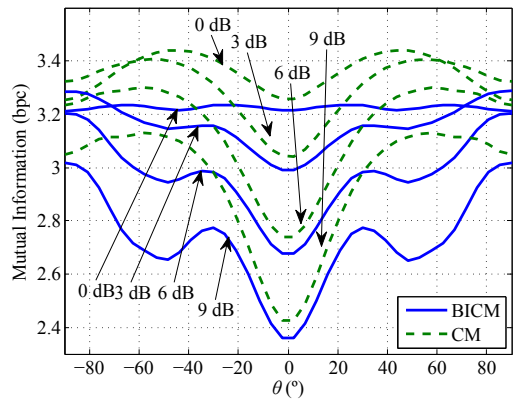

(c) 6 bpc, CR $8 / 15, \mathrm{PI} \geq 0$

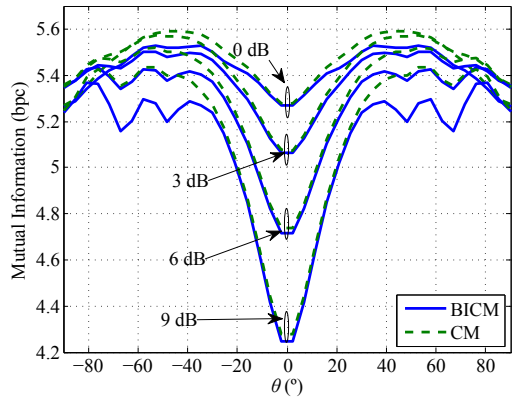

(e) 6 bpc, CR $13 / 15, \mathrm{PI} \geq 0$

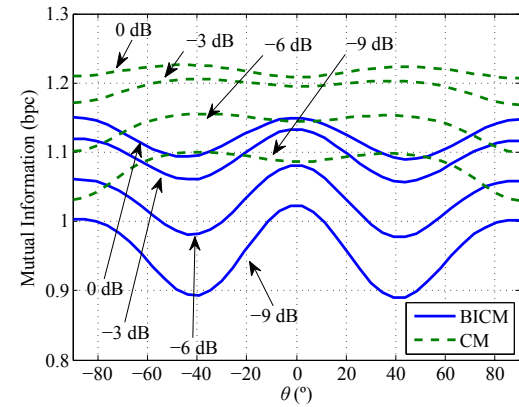

(b) 6 bpc, CR $2 / 15, \mathrm{PI} \leq 0$

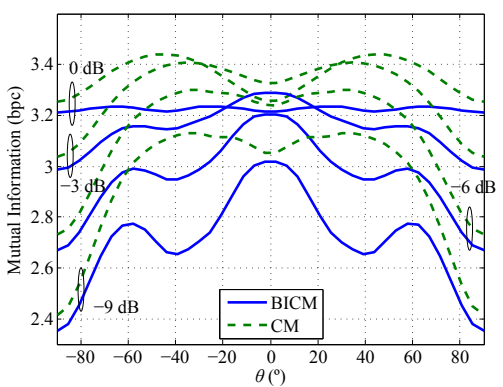

(d) 6 bpc, CR 8/15, PI $\leq 0$

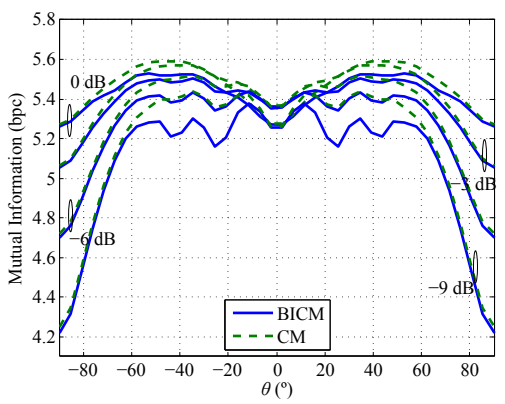

(f) $6 \mathrm{bpc}, \mathrm{CR} 13 / 15, \mathrm{PI} \leq 0$

Figure 3.15: Mutual information (bpc) vs. rotation angle $\theta\left(^{\circ}\right)$ for BICM and $\mathrm{CM}$ systems in a MIMO $2 \times 2$ with $\mathrm{XPD}=\infty$ under i.i.d. Rayleigh channel with no spatial correlation and PI with values from $P I=-9 \mathrm{~dB}$ to $P I=+9$ $\mathrm{dB}$. This figure compares the rotation performance behaviour of $6 \mathrm{bpc}$ with $P I \geq 0$ and $P I \leq 0$. 


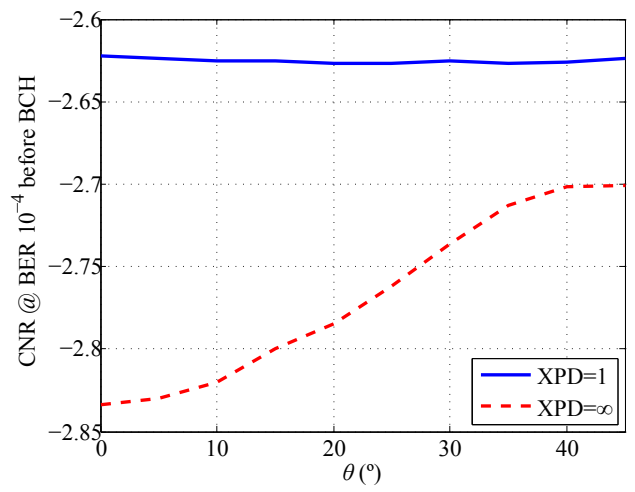

(a) 4 bpc, CR $2 / 15$

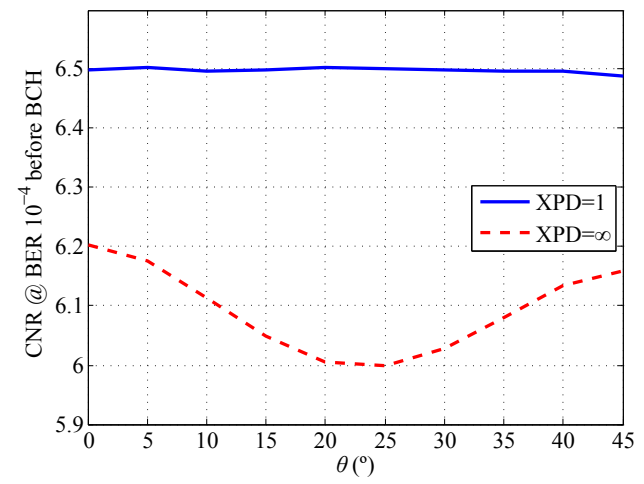

(b) 4 bpc, CR $8 / 15$

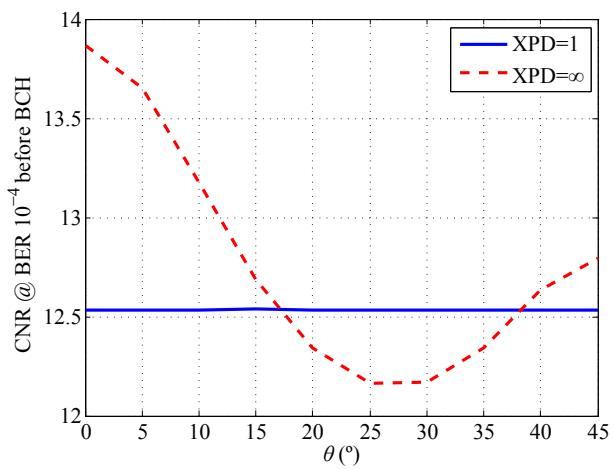

(c) 4 bpc, CR 13/15

Figure 3.16: Required CNR $(\mathrm{dB})$ at target BER $10^{-4}$ vs. rotation angle $\theta\left(^{\circ}\right)$ with $4 \mathrm{bpc}$ for MIMO $2 \times 2$ with XPD $=1$ (copolar) and XPD $=\infty$ (cross-polar) under i.i.d. Rayleigh channel with no spatial correlation and no 


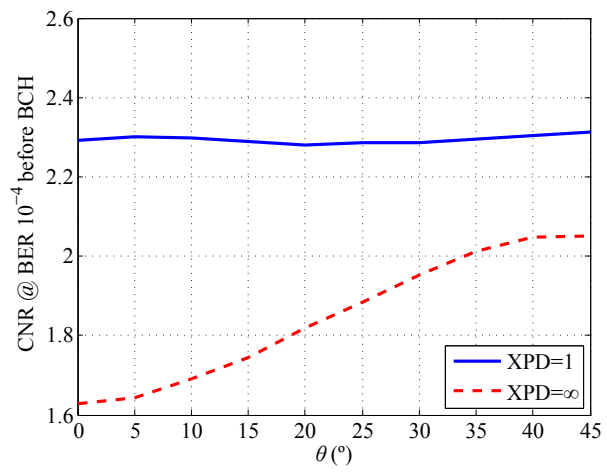

(a) 8 bpc, CR $2 / 15$

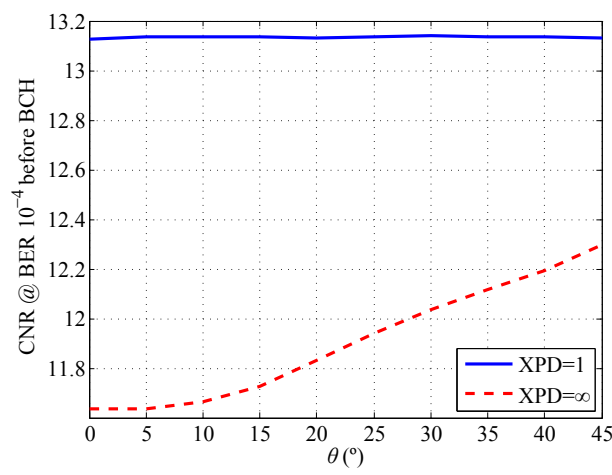

(b) 8 bpc, CR $8 / 15$

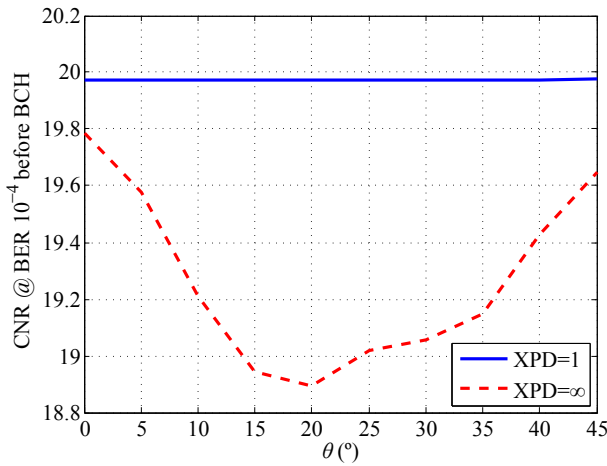

(c) 8 bpc, CR 13/15

Figure 3.17: Required CNR $(\mathrm{dB})$ at target BER $10^{-4}$ vs. rotation angle $\theta\left(^{\circ}\right)$ with $8 \mathrm{bpc}$ for MIMO $2 \times 2$ with $\mathrm{XPD}=1$ (copolar) and $\mathrm{XPD}=\infty$ (cross-polar) under i.i.d. Rayleigh channel with no spatial correlation and no 


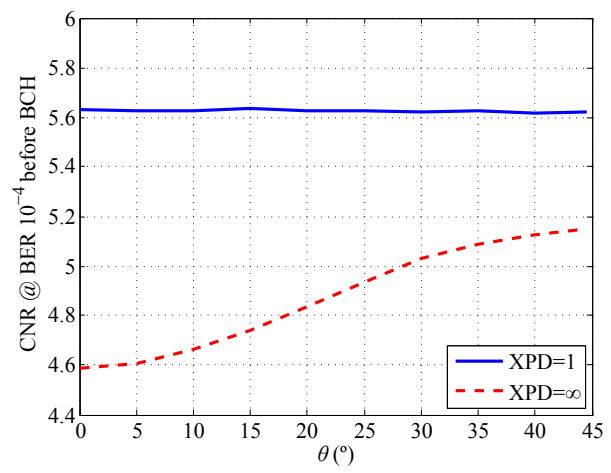

(a) 12 bpc, CR $2 / 15$

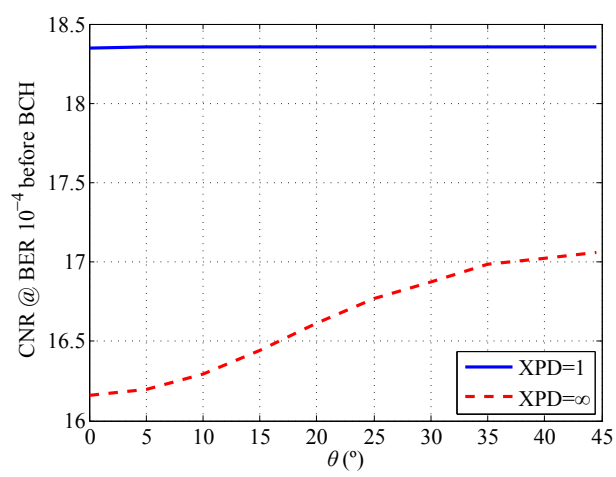

(b) 12 bpc, CR $8 / 15$

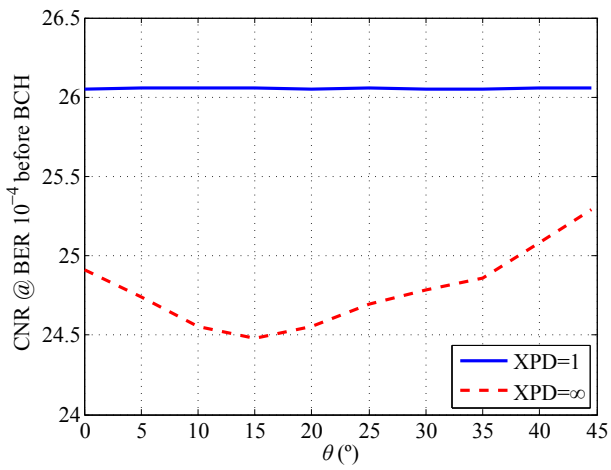

(c) $12 \mathrm{bpc}, \mathrm{CR} 13 / 15$

Figure 3.18: Required CNR $(\mathrm{dB})$ at target BER $10^{-4}$ vs. rotation angle $\theta\left(^{\circ}\right)$ with $12 \mathrm{bpc}$ for MIMO $2 \times 2$ with XPD $=1$ (copolar) and XPD= $\infty$ (cross-polar) under i.i.d. Rayleigh channel with no spatial correlation and no 


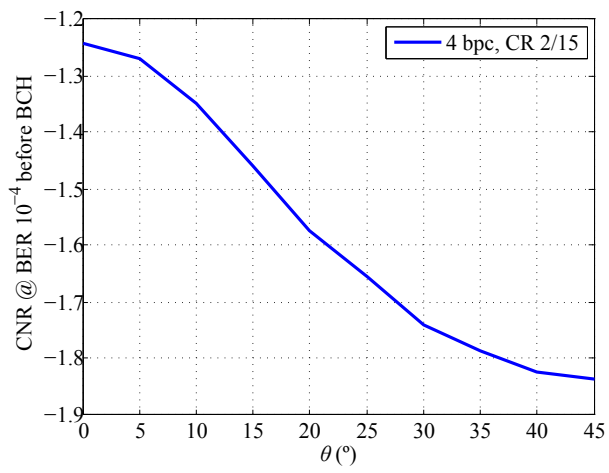

(a) 4 bpc, CR $2 / 15$

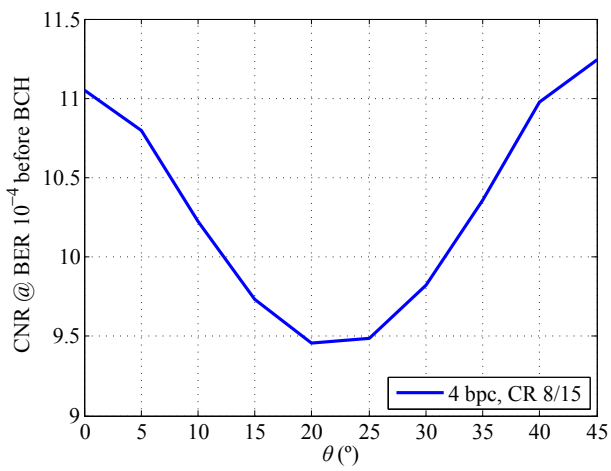

(b) 4 bpc, CR $8 / 15$

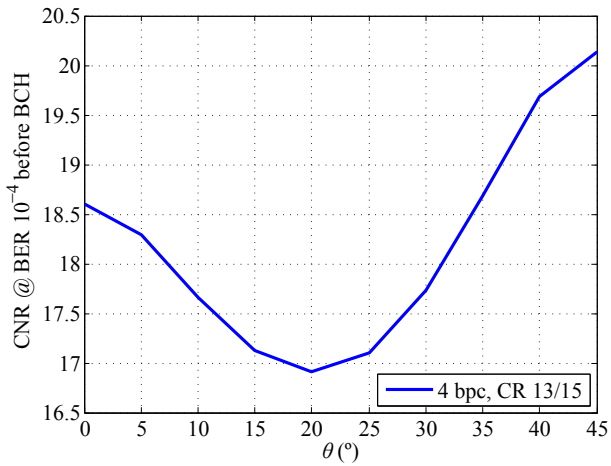

(c) 4 bpc, CR $13 / 15$

Figure 3.19: Required CNR $(\mathrm{dB})$ at target BER $10^{-4}$ vs. rotation angle $\theta\left(^{\circ}\right)$ with $4 \mathrm{bpc}$ for MIMO $2 \times 2$ with XPD $=1$ (copolar) under i.i.d. Rayleigh channel with high spatial correlation and no PI. 


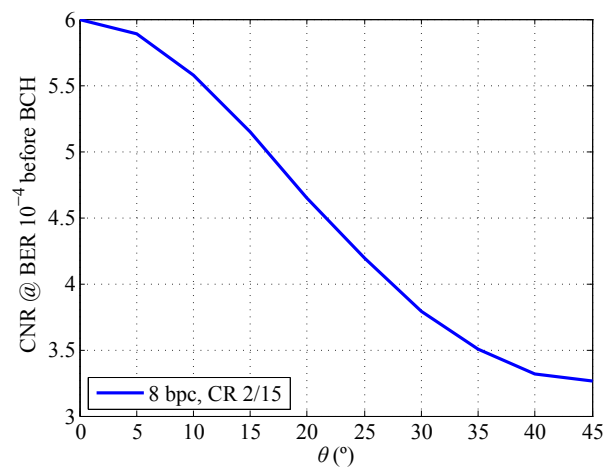

(a) 8 bpc, CR $2 / 15$

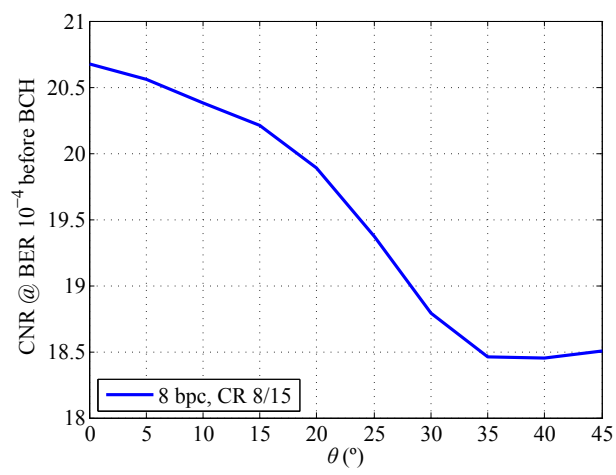

(b) 8 bpc, CR $8 / 15$

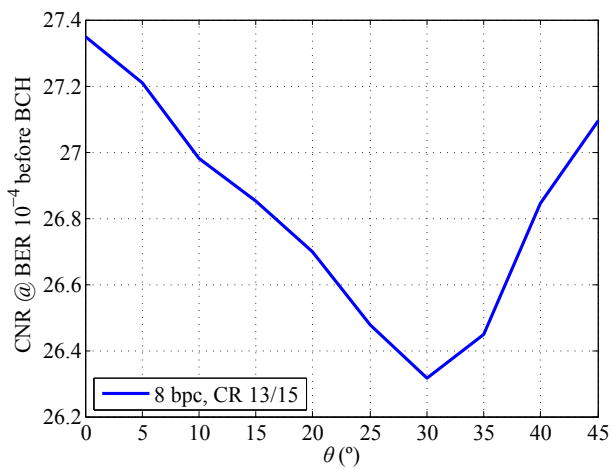

(c) 8 bpc, CR 13/15

Figure 3.20: Required CNR $(\mathrm{dB})$ at target BER $10^{-4}$ vs. rotation angle $\theta\left(^{\circ}\right)$ with 8 bpc for MIMO $2 \times 2$ with XPD $=1$ (copolar) under i.i.d. Rayleigh channel with high spatial correlation and no PI. 


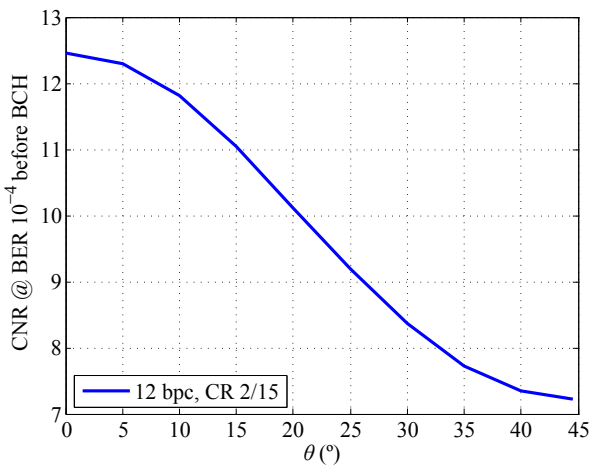

(a) 12 bpc, CR $2 / 15$

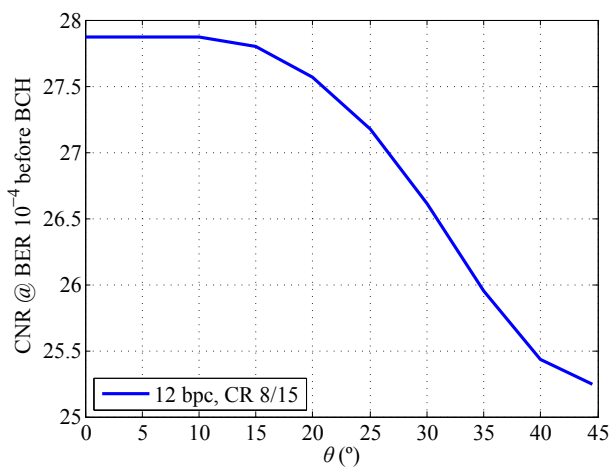

(b) 12 bpc, CR $8 / 15$

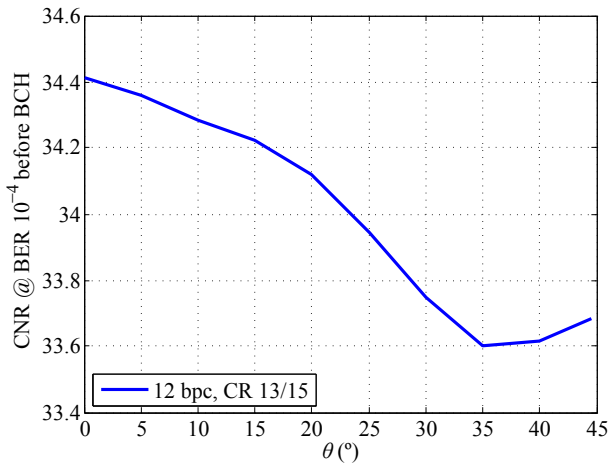

(c) 12 bpc, CR $13 / 15$

Figure 3.21: Required CNR $(\mathrm{dB})$ at target BER $10^{-4}$ vs. rotation angle $\theta\left(^{\circ}\right)$ with $12 \mathrm{bpc}$ for MIMO $2 \times 2$ with XPD $=1$ (copolar) under i.i.d. Rayleigh channel with high spatial correlation and no PI. 


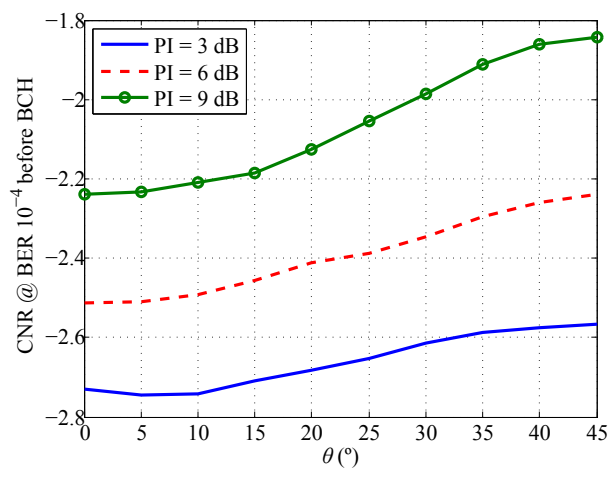

(a) 4 bpc, CR 2/15

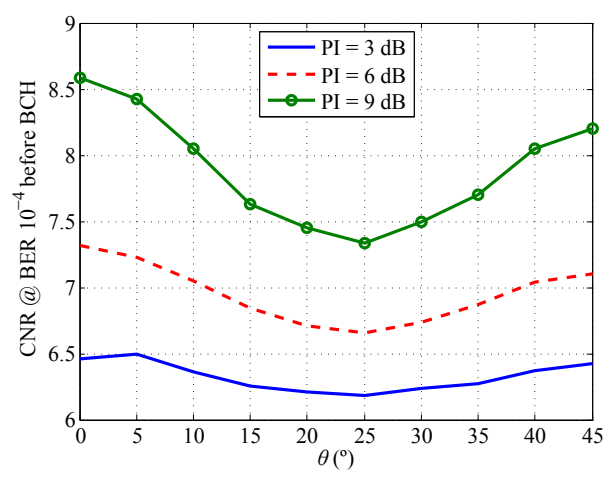

(b) 4 bpc, CR $8 / 15$

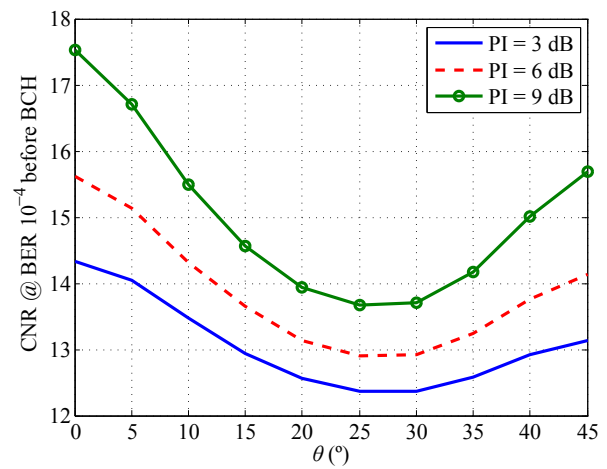

(c) 4 bpc, CR 13/15

Figure 3.22: Required CNR $(\mathrm{dB})$ at target BER $10^{-4}$ vs. rotation angle $\theta\left(^{\circ}\right)$ with $4 \mathrm{bpc}$ for MIMO $2 \times 2$ with XPD $=\infty$ (cross-polar) under i.i.d. Rayleigh channel with no spatial correlation and with PI between the transmit antennas with values of 3,6 and $9 \mathrm{dBs}$. 


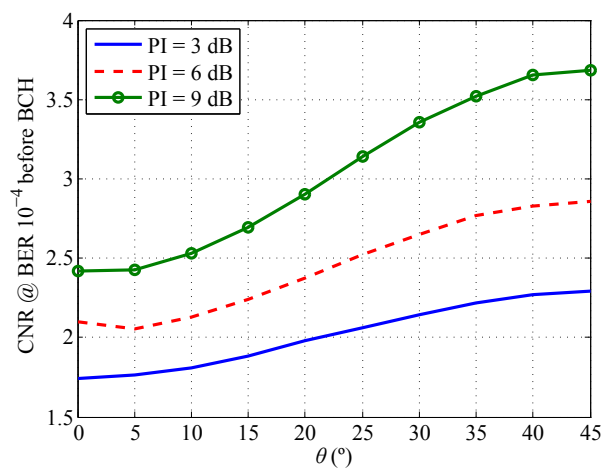

(a) 8 bpc, CR $2 / 15$

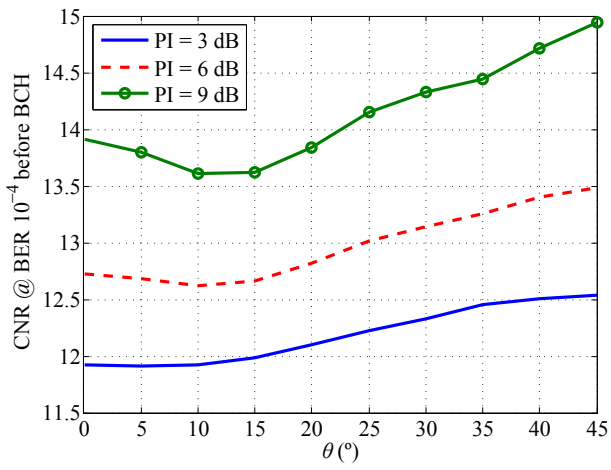

(b) 8 bpc, CR $8 / 15$

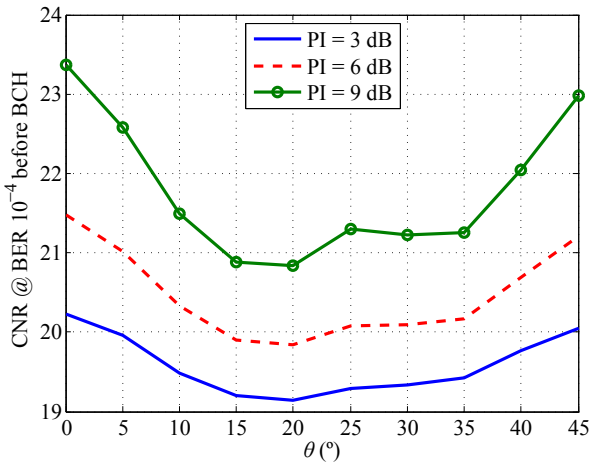

(c) 8 bpc, CR 13/15

Figure 3.23: Required CNR $(\mathrm{dB})$ at target BER $10^{-4}$ vs. rotation angle $\theta\left(^{\circ}\right)$ with 8 bpc for MIMO $2 \times 2$ with XPD $=\infty$ (cross-polar) under i.i.d. Rayleigh channel with no spatial correlation and with PI between the transmit antennas with values of 3,6 and $9 \mathrm{dBs}$. 
Table 3.1: Optimal rotation angles and CNR gains for the different transmission set-ups in subsections 3.2.1 and 3.2.2.

\begin{tabular}{|c|c|c|c|c|c|c|c|c|c|c|c|}
\hline \multirow{3}{*}{ bpc } & \multirow{3}{*}{ Code-rate } & \multirow{2}{*}{\multicolumn{2}{|c|}{$\begin{array}{c}\text { Cross-polar } \\
\text { No corr. and no PI }\end{array}$}} & \multirow{2}{*}{\multicolumn{2}{|c|}{$\begin{array}{c}\text { Co-polar } \\
\text { High corr. and no PI }\end{array}$}} & \multicolumn{6}{|c|}{$\begin{array}{l}\text { Cross-Polar } \\
\text { No Corr. }\end{array}$} \\
\hline & & & & & & & $I=3$ & & $=6$ & & $=9$ \\
\hline & & Opt. $\theta$ & Gain $(\mathrm{dB})$ & Opt. $\theta$ & Gain (dB) & Opt. $\theta$ & Gain $(\mathrm{dB})$ & Opt. $\theta$ & Gain $(\mathrm{dB})$ & Opt. $\theta$ & Gain $(\mathrm{dB})$ \\
\hline \multirow{3}{*}{4} & $2 / 15$ & 0 & 0 & 45 & 0.5941 & 5 & 0.0152 & 0 & 0 & 0 & 0 \\
\hline & $8 / 15$ & 25 & 0.2021 & 20 & 1.5875 & 25 & 0.2790 & 25 & 0.6603 & 25 & 1.2514 \\
\hline & $13 / 15$ & 25 & 1.7 & 20 & 1.6958 & 25 & 1.9626 & 25 & 2.7106 & 25 & 3.8689 \\
\hline \multirow{3}{*}{8} & $2 / 15$ & 0 & 0 & 45 & 2.7369 & 0 & 0 & 5 & 0.0450 & 0 & 0 \\
\hline & $8 / 15$ & 5 & 0.0029 & 40 & 2.2246 & 5 & 0.0050 & 10 & 0.1015 & 10 & 0.2987 \\
\hline & $13 / 15$ & 20 & 0.8919 & 30 & 1.0302 & 20 & 1.0770 & 20 & 1.6285 & 20 & 2.5254 \\
\hline \multirow{3}{*}{12} & $2 / 15$ & 0 & 0 & 44.5 & 5.2414 & - & - & - & - & - & - \\
\hline & $8 / 15$ & 0 & 0 & 44.5 & 2.6266 & - & - & - & - & - & - \\
\hline & $13 / 15$ & 15 & 0.4305 & 35 & 0.8126 & - & - & - & - & - & - \\
\hline
\end{tabular}

code-rate, any $P I \neq 0$ degrades performance. In all cases, $\theta \approx 45^{\circ}$ leads to the poorest performance. As also observed in Fig. 3.15, in the cases of $P I \geq 0$ (more power in the first transmit antenna), $\theta=90^{\circ}$ provides the best performance, while in the case of $P I \leq 0$ (more power in the second transmit antenna), $\theta=0^{\circ}$ is the optimal rotation angle. Both results lead to an increase power for the 16QAM constellation. For 8/15 and 13/15, the results also demonstrate that the best performance is provided by allocating more power to the constellation with the highest cardinality. Specifically for $8 / 15, \theta \approx 0^{\circ}$ when $P I \leq 0$, and $\theta \approx 90^{\circ}$ when $P I \geq 0$, provides the best results. For $13 / 15$, rotation can provide an improvement, and $\theta \approx 45^{\circ}$ enhances the system performance. It is interesting to note that for $8 / 15$ and $13 / 15, P I=\{ \pm 3, \pm 6\}$ can provide equal or better performance that $P I=0$ case, which can be useful in some MIMO deployments.

Here, the stream-power-allocation matrix is analysed with $6 \mathrm{bpc}$, cross-polar antennas and PI between transmit antennas. For $\alpha \neq 0.5$, the input-output relationship of the precoder is given by:

$$
\begin{array}{r}
{\left[\begin{array}{l}
x_{1} \\
x_{2}
\end{array}\right]=\sqrt{2}\left[\begin{array}{cc}
\cos \theta & \sin \theta \\
\sin \theta & -\cos \theta
\end{array}\right]\left[\begin{array}{cc}
\sqrt{\alpha} & 0 \\
0 & \sqrt{1-\alpha}
\end{array}\right]\left[\begin{array}{c}
x_{1} \\
x_{2}
\end{array}\right]=} \\
\sqrt{2}\left(s_{1} \sqrt{\alpha}\left[\begin{array}{c}
\cos \theta \\
\sin \theta
\end{array}\right]+s_{2} \sqrt{1-\alpha}\left[\begin{array}{c}
\sin \theta \\
-\cos \theta
\end{array}\right]\right),
\end{array}
$$

with expressions for the two outputs of the precoder as follows:

$$
\begin{gathered}
x_{1}=\sqrt{2}\left(s_{1} \sqrt{\alpha} \cos \theta+s_{2} \sqrt{1-\alpha} \sin \theta\right) \\
x_{2}=\sqrt{2}\left(s_{1} \sqrt{\alpha} \sin \theta-s_{2} \sqrt{1-\alpha} \cos \theta\right) .
\end{gathered}
$$

Assuming $s_{1}$ and $s_{2}$ are independent, and $E\left\{s_{1} s_{1}^{\dagger}\right\}=E\left\{s_{2} s_{2}^{\dagger}\right\}=1$, then the power at the first transmit antenna is:

$$
P_{1}=\alpha \cos ^{2} \theta+(1-\alpha) \sin ^{2} \theta=\alpha\left(\cos ^{2} \theta-\sin ^{2} \theta\right)+\sin ^{2} \theta,
$$




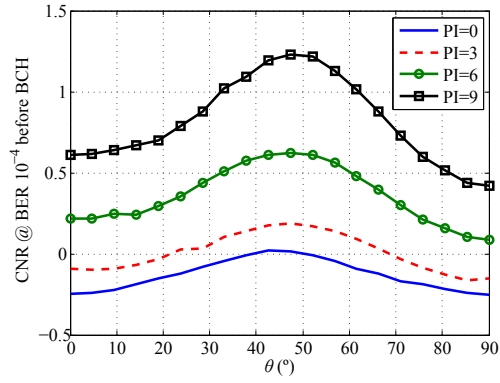

(a) 6 bpc, CR 2/15, PI $\geq 0$

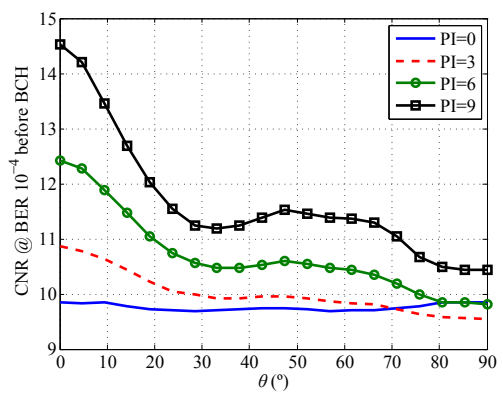

(c) 6 bpc, CR 8/15, PI $\geq 0$

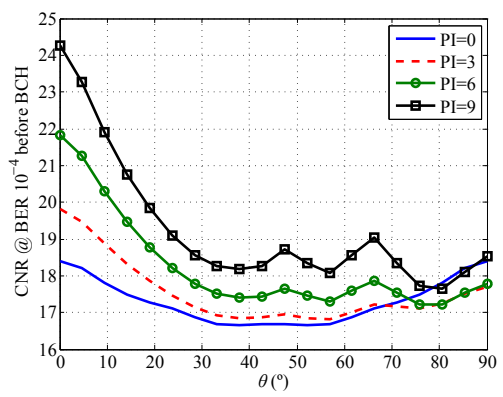

(e) 6 bpc, CR 13/15, PI $\geq 0$

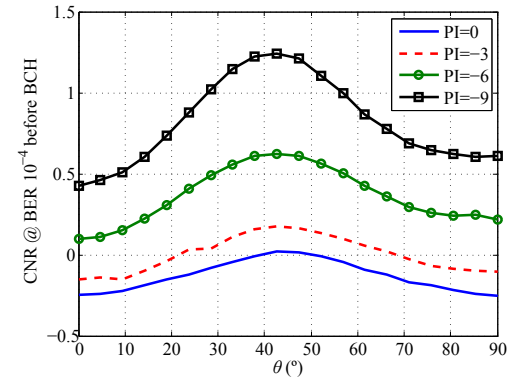

(b) 6 bpc, CR $2 / 15, \mathrm{PI} \leq 0$

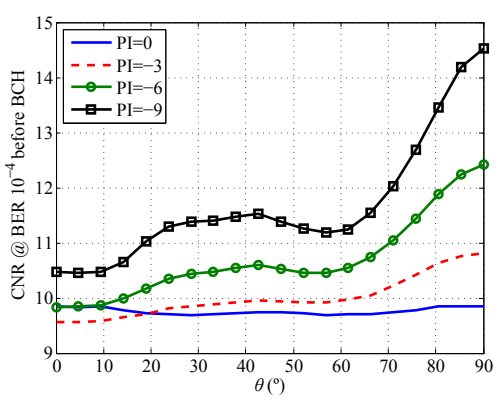

(d) $6 \mathrm{bpc}, \mathrm{CR} 8 / 15, \mathrm{PI} \leq 0$

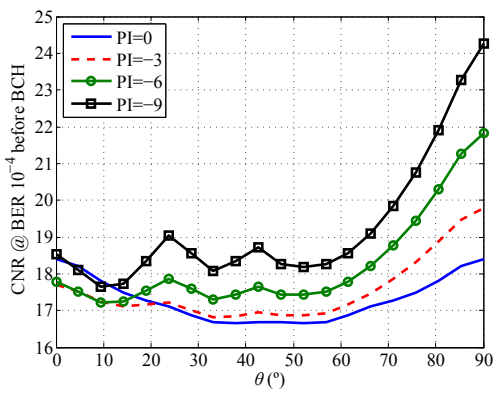

(f) $6 \mathrm{bpc}, \mathrm{CR} 13 / 15, \mathrm{PI} \leq 0$

Figure 3.24: Required CNR $(\mathrm{dB})$ at target BER $10^{-4}$ vs. rotation angle $\theta\left(^{\circ}\right)$ for MIMO $2 \times 2$ with XPD $=\infty$ (cross-polar) under i.i.d. Rayleigh channel with no spatial correlation and with PI between the transmit antennas with values from $P I=-9 \mathrm{~dB}$ to $P I=+9 \mathrm{~dB}$. This figure compares rotation performance behaviour of $6 \mathrm{bpc}$ with $P I \geq 0$ and $P I \leq 0$. 
and similarly the power at the second transmit antenna is:

$$
P_{2}=\alpha \sin ^{2} \theta+(1-\alpha) \cos ^{2} \theta=\alpha\left(\sin ^{2} \theta-\cos ^{2} \theta\right)+\cos ^{2} \theta
$$

In the case where the same power is allocated to the two streams at the output of the precoder $\left(P_{1}=P_{2}\right)$, hence:

$$
\begin{array}{r}
\alpha\left(\cos ^{2} \theta-\sin ^{2} \theta\right)+\sin ^{2} \theta-\alpha\left(\sin ^{2} \theta-\cos ^{2} \theta\right)-\cos ^{2} \theta= \\
\left(\cos ^{2} \theta-\sin ^{2} \theta\right)(2 \alpha-1)=0,
\end{array}
$$

which leads to two possible solutions:

$$
\begin{aligned}
& \text { - } 2 \alpha-1=0, \quad \alpha=1 / 2, \quad \forall \theta, \\
& \text { - } \tan \theta= \pm 1, \quad \theta= \pm \pi / 4+k \pi, \quad k=\{\ldots,-1,0,1, \ldots\} \quad \forall \alpha .
\end{aligned}
$$

The first solution is trivial, since it removes the effect of the stream-powerallocation matrix and leaves the precoder with the rotation matrix only. With $\alpha=0.5$, for all possible values of $\theta$, the two streams at the output of the precoder have the same power. For the second solution, equal power at the two outputs of the precoder is achieved with $\theta= \pm \pi / 4+k \pi, \quad k=\{\ldots,-1,0,1, \ldots\}$, independently of the $\alpha$ value selected. This configuration is interesting since it allows to design the power allocated to each of the input information streams $s_{1}$ and $s_{2}$ without unbalancing the power at each transmit antenna.

Fig. 3.25 shows the CNR requirements vs. the ratio $P_{s_{1}} / P_{s_{2}}(\mathrm{~dB})$ for $6 \mathrm{bpc}$. The $\theta$ is selected to the value $45^{\circ}$, which provides same transmitted power in both information streams at the output of the precoder. For low code-rate 2/15, it is optimal to allocate the same power to both streams. For mid and high code-rates, one can observe that $P_{s_{1}} / P_{s_{2}}=-3 \mathrm{~dB}$ provides the best results. The improvement over equal power stream allocation with $P I=0 \mathrm{~dB}$ is 0.1 and $0.4 \mathrm{dBs}$ for CRs $8 / 15$ and $13 / 15$, respectively. It is also noted, that a PI between transmit antennas of either $\pm 3 \mathrm{dBs}$ provides the same performance. This can be explained by inspection of expression (3.3), which for a value of $\theta=45^{\circ}$ the same ratio $P_{s_{1}} / P_{s_{2}}$ is available at both transmit antennas.

\subsubsection{MIMO Precoding in DVB-NGH and ATSC 3.0}

The MIMO profiles of DVB-NGH and ATSC 3.0 have precoding based on rotation matrices as the one studied in the previous subsections. In this subsection, first the precoder components selected for each standard will be presented. Additionally, the selection of the precoding parameters in DVB-NGH and ATSC 3.0 is discussed and compared with the results obtained in the previous subsection. 


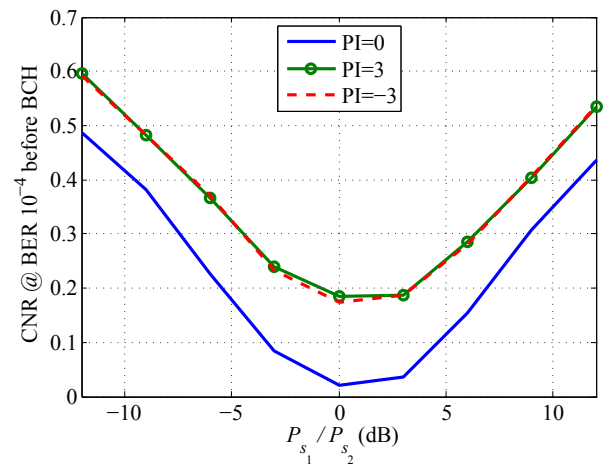

(a) 6 bpc, CR $2 / 15$

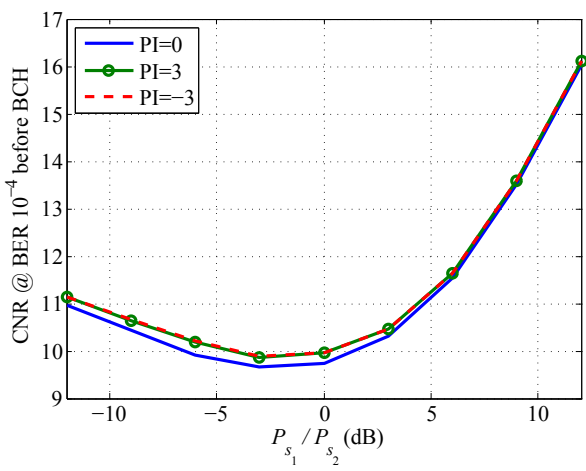

(b) 6 bpc, CR $8 / 15$

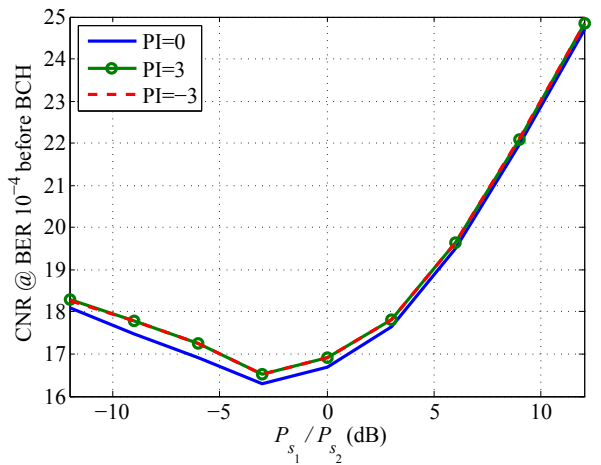

(c) 6 bpc, CR 13/15

Figure 3.25: Required CNR $(\mathrm{dB})$ at target BER $10^{-4}$ vs. $P_{s_{1}} / P_{s_{2}}(\mathrm{~dB})$ for MIMO $2 \times 2$ with XPD $=\infty$ (cross-polar) with 6 bpc under i.i.d. Rayleigh channel with no spatial correlation and with PI between the transmit antennas. 
Table 3.2: eSM Parameters for DVB-NGH

\begin{tabular}{|c|c|c|c|c|c|c|c|c|c|c|c|}
\hline \multicolumn{3}{|c|}{$\begin{array}{c}\text { Deliberate Power Imbalance } \\
\text { Between two Tx Antennas }\end{array}$} & \multicolumn{3}{|c|}{$0 \mathrm{~dB}$} & \multicolumn{3}{|c|}{$3 \mathrm{~dB}$} & \multicolumn{3}{|c|}{$6 \mathrm{~dB}$} \\
\hline $\mathrm{bpc}$ & & dulation & $\beta$ & $\theta$ & $\alpha$ & $\beta$ & $\theta$ & $\alpha$ & $\beta$ & $\theta$ & $\alpha$ \\
\hline 6 & $x_{1}$ & $\begin{array}{c}\text { QPSK } \\
16 \mathrm{AMM}\end{array}$ & 0.5 & $45.0^{\circ}$ & 0.44 & \multirow[t]{2}{*}{$1 / 3$} & $0.0^{\circ}$ & 0.50 & \multirow[t]{2}{*}{0.20} & $0.0^{\circ}$ & 0.50 \\
\hline \multirow{2}{*}{8} & $x_{1}$ & 16QAM & \multirow{2}{*}{0.50} & \multirow{2}{*}{$57.8^{\circ}$} & \multirow[b]{2}{*}{0.50} & & \multirow{2}{*}{$25.0^{\circ}$} & \multirow{2}{*}{0.50} & & \multirow{2}{*}{$0.0^{\circ}$} & \multirow{2}{*}{0.50} \\
\hline & $x_{2}$ & 16QAM & & & & $1 / 3$ & & & 0.20 & & \\
\hline \multirow[t]{2}{*}{10} & $x_{1}$ & 16QAM & \multirow[t]{2}{*}{0.50} & \multirow[t]{2}{*}{$22.0^{\circ}$} & \multirow[t]{2}{*}{0.50} & \multirow[t]{2}{*}{$1 / 3$} & \multirow[t]{2}{*}{$15.0^{\circ}$} & \multirow{2}{*}{0.50} & \multirow[t]{2}{*}{0.20} & \multirow[t]{2}{*}{$0.0^{\circ}$} & \multirow[t]{2}{*}{0.50} \\
\hline & $x_{2}$ & 64QAM & & & & & & & & & \\
\hline
\end{tabular}

MIMO precoding in DVB-NGH The precoding adopted in the MIMO profile of DVB-NGH, named eSM-PH, has the structure of (3.1) and an additional phase hopping term with complete mathematical expression as follows:

$$
\begin{aligned}
{\left[\begin{array}{l}
x_{1} \\
x_{2}
\end{array}\right]=2 } & \overbrace{\left[\begin{array}{cc}
1 & 0 \\
0 & e^{j \phi(i)}
\end{array}\right]}^{\text {phase hopping }}\left[\begin{array}{cc}
\sqrt{\beta} & 0 \\
0 & \sqrt{1-\beta}
\end{array}\right]\left[\begin{array}{cc}
\cos \theta & \sin \theta \\
\sin \theta & -\cos \theta
\end{array}\right] . \\
& \cdot\left[\begin{array}{cc}
\sqrt{\alpha} & 0 \\
0 & \sqrt{1-\alpha}
\end{array}\right]\left[\begin{array}{l}
s_{1} \\
s_{2}
\end{array}\right], \quad i=0, \ldots, \frac{N_{\text {data }}}{2}-1 .
\end{aligned}
$$

where $i$ is the QAM symbol index and $N_{\text {data }}$ is the number of QAM symbols corresponding to one LDPC code word for a given modulation order.

In addition to the PI, rotation and stream-power-allocation matrices considered in (3.1), eSM-PH includes a phase-hoping term $e^{j \phi(i)}$ where $\phi(i) \in[0,2 \pi)$. Phase-hopping improves the performance by avoiding any specific worst case channel condition over various channel realizations by a progressive phase change at the second transmit antenna. Although using phase hopping may slightly degrade the performance under some specific channel conditions, it improves overall performance by improving the worst channel condition. It was confirmed by numerical simulations that, in the i.i.d. Rayleigh channels considered in this section, phase hopping does not have any effect in the system performance.

In DVB-NGH the PI matrix is considered as part of the precoder and was introduced to ease cross-polar operation in MIMO and SISO networks. The eSM-PH scheme can be transmitted with deliberated PI between the transmit antennas with values of $0 \mathrm{~dB}, 3 \mathrm{~dB}$ and $6 \mathrm{~dB}$. This deliberated transmitted PI was motivated to facilitate deployment scenarios where not all the receiver population supports MIMO, since it provides a reasonable coverage reduction for SISO/SIMO terminals. Additionally, eSM-PH was optimized to maintain good performance under imbalance cases. 
The parameters $\beta, \theta$ and $\alpha$ of the eSM precoder are summarized in Table 3.2. Multiple remarks can be made by comparing the parameters selected in DVBNGH and the results obtained in the previous sections:

- DVB-NGH optimizes $\beta, \theta$, and $\alpha$ for the three cases of bpc (i.e., 6,8 and $10 \mathrm{bpc}$ ) and for the different values of transmit PI. It is important to note that these parameters are to be used for every code-rate available in the MIMO profile of DVB-NGH. However, as it has been investigated in previous sections, there is a strong dependency of the optimal rotation angle with the code-rate, which makes the precoding potentially suboptimal for some transmit configurations.

- The parameter $\alpha$ has for all the combinations of Table 3.2 the value 0.5 , except for the $6 \mathrm{bpc}$ configuration with $P I=0$ in which $\alpha=0.44$. The value $\alpha=0.5$ transforms the stream-power-allocation matrix to the identity matrix, which has no effect in the precoder. Interestingly, in the case $\alpha=0.44$, the rotation parameter $\theta$ takes the value $45^{\circ}$, which as we saw in subsection 3.2.2 provides equal power at the two transmit antennas (but prior to the PI matrix). Furthermore, the value $\alpha=0.44$ assigns more power to the data stream mapped to a 16QAM constellation than the data stream mapped to QPSK constellation, which agrees with the results of in Fig. 3.25.

- Since $\alpha$ is always 0.5, except for the case discussed in the previous bullet point, the parameter $\beta$ takes the values $0.5,0.33$, and 0.2 to provide PIs between transmit antennas of 0,3 and $6 \mathrm{dBs}$, respectively.

MIMO precoding in ATSC 3.0 The MIMO precoding scheme adopted in ATSC 3.0 is composed of a rotation matrix, an I\&Q polarization interleaver and a phase-hopping blocks [15]. Each of the three sub-blocks of the MIMO precoder can be optionally selected. The phase-hopping block adopted in ATSC 3.0 is the same as in DVB-NGH and discussed in previous section and detailed in Appendix B. The I\&Q polarization interleaver is simply a switching interleaving operation, such that the output cells consist of the real (In-phase) component of one input symbol and the imaginary (Quadrature) component of the other input symbol. The I/Q polarization interleaving is described by the following equations:

$$
\begin{gathered}
x_{1}=\operatorname{Re}\left\{s_{1}\right\}+i \operatorname{Im}\left\{s_{2}\right\} \\
x_{2}=\operatorname{Re}\left\{s_{2}\right\}+i \operatorname{Im}\left\{s_{1}\right\} .
\end{gathered}
$$

The rotation matrix has the same form as the one discussed in detail in this chapter and also included in DVB-NGH. The value of the rotation angle $\theta$ is fixed and its value depends on the modulation and code-rate used. 
Table 3.3: Rotation angles and gains $(\mathrm{dB})$ compared to no rotation for the constellations and code-rates selected for ATSC 3.0 system.

\begin{tabular}{|c|c|c|c|c|c|c|}
\hline Code-Rate & $4 \mathrm{bpc}$ & Rotation Gain $(\mathrm{dB})$ & $8 \mathrm{bpc}$ & Rotation Gain $(\mathrm{dB})$ & $\begin{array}{c}12,16, \\
\text { 20, and 24 bpc }\end{array}$ & Rotation Gain (dB) \\
\hline $2 / 15$ & $0^{\circ}$ & 0 & $0^{\circ}$ & 0 & $0^{\circ}$ & 0 \\
\hline $3 / 15$ & $0^{\circ}$ & 0 & $0^{\circ}$ & 0 & $0^{\circ}$ & 0 \\
\hline $4 / 15$ & $0^{\circ}$ & 0 & $0^{\circ}$ & 0 & $0^{\circ}$ & 0 \\
\hline $5 / 15$ & $0^{\circ}$ & 0 & $0^{\circ}$ & 0 & $0^{\circ}$ & 0 \\
\hline $6 / 15$ & $15^{\circ}$ & 0.02 & $0^{\circ}$ & 0 & $0^{\circ}$ & 0 \\
\hline $7 / 15$ & $15^{\circ}$ & 0.17 & $0^{\circ}$ & 0 & $0^{\circ}$ & 0 \\
\hline $8 / 15$ & $20^{\circ}$ & 0.22 & $0^{\circ}$ & 0 & $0^{\circ}$ & 0 \\
\hline $9 / 15$ & $20^{\circ}$ & 0.53 & $0^{\circ}$ & 0 & $0^{\circ}$ & 0 \\
\hline $10 / 15$ & $25^{\circ}$ & 0.83 & $0^{\circ}$ & 0 & $0^{\circ}$ & 0 \\
\hline $11 / 15$ & $25^{\circ}$ & 1.1 & $15^{\circ}$ & 0.11 & 0.27 & $0^{\circ}$ \\
\hline $12 / 15$ & $25^{\circ}$ & 1.34 & $15^{\circ}$ & $15^{\circ}$ & 0.35 & 0 \\
\hline $13 / 15$ & $30^{\circ}$ & 1.51 & & & 0 \\
\hline
\end{tabular}

ATSC 3.0 specifies a wide set of possible transmit constellations ranging from 4 bpc (QPSK in each transmit antenna) to 24 bpc (4096QAM in each transmit antenna). In contrast to DVB-NGH, it does not include the option of transmitting constellations with different cardinality in each transmit antenna. It is important to note that the constellations adopted in ATSC 3.0 for the MIMO profile are non-uniform constellations (cf. Chapter 2), although the constellations have been reused from the SISO profile and no specific optimization was performed for the MIMO case.

The $\theta$ values for the different constellations and code-rates available for the MIMO profile of ATSC 3.0 are summarized in Table 3.3 and extracted from [15]. The angles showed in Table 3.3 were optimized using a complete end-to-end physical layer MIMO ATSC 3.0 simulation platform including the adopted LDPC codes, bit-interleavers and constellations in the specification. Two channel models were used in the optimization of the rotation angle, the mobile NGH channel model (for 4 and $8 \mathrm{bpc}$ ) and the rooftop reception Modified Guilford Model (MGM) channel model (for 12 to $24 \mathrm{bpc}$ ). Both channels extracted from measurement campaigns in the UHF band with $2 \times 2$ MIMO cross-polar antennas. These two channel models are presented in detail in the Appendix B of this thesis and will be also used in further evaluations. The $\theta$ values selected for ATSC 3.0 as optimal angle provide the highest CNR gain in the case of $P I=9 \mathrm{~dB}$. The rotation angles for spectral efficiencies from 12 to $24 \mathrm{bpc}$ show an optimal rotation angle always equal to zero degrees. These results were obtained using a MMSE demapper, which provides linear demapping complexity with reasonable performance (cf. Chapter 2). This type of demodulator was selected to reduce the very high computational complexity of demapping spectral efficiencies such as $24 \mathrm{bpc}$. Hence, the results for the optimal rotation angle could be different if a different demapping algorithm is selected, e.g., MAP or sphere decoding techniques. 
Comparing the results in Table 3.3 with the investigations about rotation angle in this thesis the following remarks are provided:

- ATSC 3.0 uses a different rotation angle per code-rate, which has been shown in this chapter to be optimal due to strong relationship between the rotation performance and the code-rate.

- The results in Table 3.3 show that: (i) the rotation gain increases with the code-rate, (ii) for low to mid code-rates it is optimal to rotate with zero degrees angle, and (iii) the gain is higher for lower order constellations. These results are very well aligned with the results shown in section 3.2.1 and section 3.2.2 with i.i.d. Rayleigh channel with cross-polar antennas. The mobile NGH channel model is a cross-polar channel with high XPD and low spatial correlation, which makes a similar scenario to the one studied in this chapter.

- The results in Table 3.3 reinforce the conclusions extracted in this chapter since even with different physical layer components, such as bitinterleavers and constellations, and even different channel models, the conclusions still hold.

\subsection{Precoding Based on Maximum Ergodic Ca- pacity}

Due to the lack of feedback channel from the receiver to the transmitter, and differing channel realizations at different locations of the broadcasting network, conventional MIMO-precoding that maximizes capacity of individual MIMO links cannot be employed in the broadcasting system. On the contrary, the precoding design proposed in this part of the chapter exploits common statistical structure found in the overall broadcast network such as statistical distribution of the channel, correlation between antennas, and LoS conditions. This precoder design aims to maximize the ergodic capacity of the MIMO broadcasting system and depends only on the channel model and the target $\mathrm{CNR}^{1}$.

First, the ergodic capacity of MIMO channel with no information at the transmitter, perfect CSI at the receiver and zero-mean Gaussian distributed inputs is recalled as [56]:

$$
C=E_{\mathbf{H}}\left\{\log _{2} \operatorname{det}\left(\mathbf{I}_{N_{r}}+\frac{\rho}{N_{t}} \mathbf{H} \mathbf{H}^{\dagger}\right)\right\},
$$

\footnotetext{
${ }^{1}$ The main results of this section are covered in [96]. (C) 2015 IEEE.
} 
where $\rho$ is the CNR in linear units, $\mathbf{I}_{N_{r}}$ is the identity matrix of size $N_{r} \times N_{r}$, and the statistical expectation operator $E$ is over all possible channel realizations. Equation (3.9) provides with the maximum achievable system rate with diminishing error probability as the transmission duration tends to infinity (cf. Chapter 2). This definition is convenient for fast fading channels or for long codeword transmission in which the channel can be assumed to be sufficiently averaged.

The previous definition assumed perfect CSI at the receiver with no information at the transmitter. However, the broadcast network tends to exhibit common channel characteristics such as predominant LoS (i.e., high $K$ factor) in rooftop environment, or correlation between antenna paths [17]. Inspired by $[56,48,50,49,51]$, a MIMO channel-precoder is designed that attempts to adapt the transmission signal characteristics to the channel statistics to increase the ergodic capacity in MIMO DTT systems. The approach followed in this chapter of exploiting the channel statistics can provide significant capacity improvements for users with strong LoS component and/or correlation among antennas, while preserving similar area coverage for receivers with dominant multipath environment, i.e., low $K$ factor, and uncorrelated antenna paths. The optimization problem is mathematically defined as:

$$
\begin{aligned}
& \underset{\mathbf{Q} \succeq 0 \text { s.t. }}{\operatorname{maximize}} \quad E_{\mathbf{H}}\left\{\log _{2} \operatorname{det}\left(\mathbf{I}_{N_{r}}+\frac{\rho}{N_{t}} \mathbf{H Q H}^{\dagger}\right)\right\} \\
& \operatorname{trace}(\mathbf{Q})=N_{t}
\end{aligned}
$$

where the statistical expectation is over all realizations of MIMO channel $\mathbf{H}$, and $\mathbf{Q}$ is the covariance matrix of the transmitted vector $\mathbf{x}$. While the first constraint keeps the positive semi-definite property of the covariance matrix, the second constraint maintains constant sum power for any transmit antenna dimension, i.e., trace $(\mathbf{Q}) / N_{t}=1$. With strong error correcting codes, such as LDPC codes used in the considered MIMO system, capacity optimization criterion is the preferred metric [50].

Once the capacity maximizing $\mathbf{Q}$ is obtained from (3.10), it can be further decomposed into $\mathbf{Q}=\mathbf{U} \Lambda \mathbf{U}^{\dagger}$ by the eigen-decomposition [97], where $\mathbf{U}$ is the unitary matrix whose columns are the eigenvectors of $\mathbf{Q}$, and $\Lambda$ is the diagonal matrix whose diagonal entries are the corresponding non-negative real eigenvalues. Consequently, the optimal channel-precoder which maximizes the system ergodic capacity is given by:

$$
\Gamma=\mathbf{U} \Lambda^{\frac{1}{2}}
$$

and the information streams are precoded as:

$$
\mathbf{x}_{\mathrm{p}}=\Gamma \mathbf{x},
$$


where $\Gamma$ is the channel-precoder matrix, and $\mathbf{x}$ and $\mathbf{x}_{\mathrm{p}}$ are input/output symbol vectors to the channel-precoder.

With the precoding, the power per transmit antenna is given by $\operatorname{diag}\left(E\left\{\mathbf{x}_{p} \mathbf{x}_{p}^{\dagger}\right\}\right)$ where

$$
\begin{array}{r}
E\left\{\mathbf{x}_{p} \mathbf{x}_{p}^{\dagger}\right\}=E\left\{\Gamma \mathbf{x} \mathbf{x}^{\dagger} \Gamma^{\dagger}\right\}=\Gamma E\left\{\mathbf{x} \mathbf{x}^{\dagger}\right\} \Gamma^{\dagger} \\
=\Gamma \Gamma^{\dagger}=\mathbf{U} \boldsymbol{\Lambda}^{\frac{1}{2}} \Lambda^{\frac{1}{2}} \mathbf{U}^{\dagger}=\mathbf{Q}
\end{array}
$$

because for i.i.d. column vector $\mathbf{x}, E\left\{\mathbf{x x}^{\dagger}\right\}=\mathbf{I}_{N_{t}}$. Thus, the power allocation per transmit antenna in this precoded MIMO system is given by $\operatorname{diag}(\mathbf{Q}) / N_{t}$. Consequently, this channel-precoding allocates different power per transmit antenna. However, for all the solutions proposed in this part of the chapter, the maximum power imbalance between any pair of transmit antennas is lower than $0.5 \mathrm{~dB}$, that can be considered negligible.

Equation (3.10) describes a convex optimization problem because log-determinant is a concave function over positive semi-definite matrices and expectation is a linear operator. Hence, the optimal value can be calculated numerically by using standard convex optimization techniques [98]. Direct computation of the optimization problem, however, is still computationally expensive due to the large degrees of freedom in the MIMO-channel matrix $\mathbf{H}$ found in the broadcasting systems. Consequently, next a semi-analytical solution is proposed below with low computational complexity, to obtain MIMO channel-precoders based on ergodic capacity ${ }^{2}$ for a generic MIMO transmission system of dimension $N_{t} \times N_{r}$.

The system model employed in this part of the chapter is based on DVBNGH physical layer standard specification and presented in Fig. 3.26. The following subsections study two transmitter configurations with two and four transmit aerials and receivers with two receive aerials, i.e., MIMO $2 \times 2$ and MIMO $4 \times 2$ systems, respectively. While the two transmit antennas case is included in DVB-NGH standard, the four transmit antennas case is an extension of DVB-NGH physical layer.

\footnotetext{
${ }^{2}$ For the case of quasi-static or slow fading, in which one codeword is affected by one channel realization, the appropriate measure is the $\epsilon$-outage capacity with the following expression: $C_{\epsilon} \triangleq \sup \left\{R \mid \operatorname{Pr}\left\{C_{\mathbf{H}}<R\right\}<\epsilon\right\}$ where $C_{\mathbf{H}}$ is the capacity of a specific channel realization, and $\operatorname{Pr}\left\{C_{\mathbf{H}}<R\right\}$ is the probability that $C_{\mathbf{H}}$ is lower than rate $R$. The $\epsilon$-outage capacity can be interpreted as the minimum rate $C_{\epsilon}$ that can be achieved at the $(1-\epsilon) 100 \%$ of the channel realizations (cf. Chapter 2). The optimization of channel-precoders based on outage capacity requires a different approach to the one proposed in this thesis and is thus beyond the scope of this dissertation. For the interested reader references [99] and [100] provide results related to the optimization of transmission techniques based on outage capacity.
} 


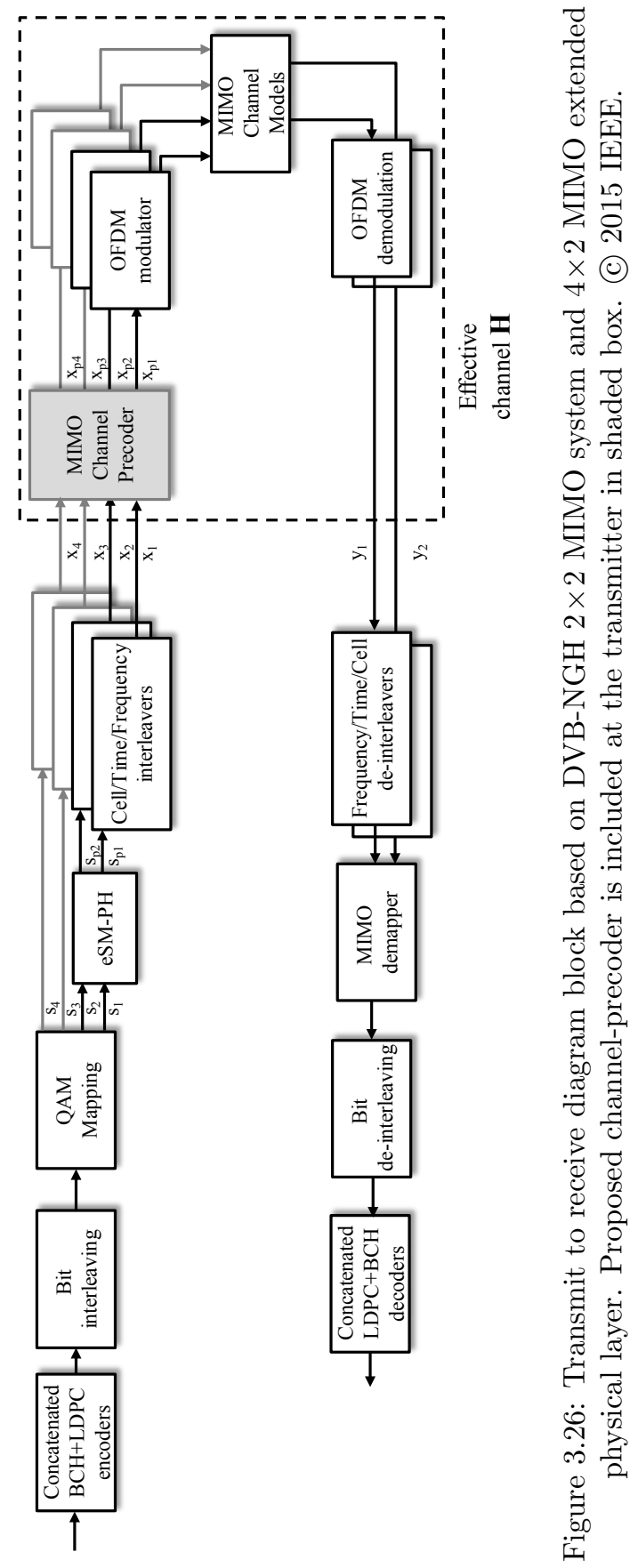




\section{CHAPTER 3. MIMO PRECODING FOR TERRESTRIAL BROADCAST SYSTEMS}

The incoming bit stream is first encoded by the concatenation of a $\mathrm{BCH}$ and LDPC codes and passed through the bit-interleaver that, specifically for DVBNGH MIMO, it was designed to exploit the quasi-cyclic structure of the LDPC codes exhibiting low complexity, low latency, and fully parallel design easing the implementation of iterative structures (cf. Appendix B). The interleaved code bits are then multiplexed into one data stream (layer) per transmit antenna following a Gray labelling. Subsequently, in the case of two transmit antennas, the modulated data streams are processed by the eSM-PH processing block (cf. subection 3.2.3). In Fig. $3.26 s_{1}, s_{2}, s_{p 1}$, and $s_{p 2}$ are the input/output constellation symbols to the eSM-PH precoding where both transmit antennas have the same power, i.e., $P I=0$. In case of four transmit antennas, the transmitter spatially multiplexes the four modulated data streams $s_{1}, s_{2}, s_{3}, s_{4}$ which are passed directly to the cell interleaver operating at codeword level. The cell interleaver applies a different pseudo-random permutation for every codeword to ensure a uniform distribution of the channel fading realizations. Then, the time interleaver interlaces symbols from several codewords over various OFDM symbols to provide protection against selective fading. After time interleaving, the frequency interleaver operates on an OFDM level and its function is twofold. First it mixes up symbols from various services and secondly, it applies a pseudo-random permutation to break the structured nature of the time interleaver output. Here, the proposed MIMO channel-precoder gives the option of combining the samples among transmit layers according to a specific channelprecoding matrix per OFDM carrier as in (3.12). Finally, before transmission across the cross-polarized antennas, the signal is passed from frequency to time domain by IFFT operation plus guard interval insertion, which composes the OFDM modulator.

The channel models used are the NGH portable outdoor channel model and the MGM channel model, which represent a portable outdoor and fixed rooftop reception environments. A detailed description of the channel models and its extension to four transmit antennas is presented in Appendix A of this dissertation.

The signal distorted by the channel is received by two cross-polarized antennas. Referring to Fig. 3.26, the received streams are first processed by the OFDM demodulator, which essentially discards the guard interval and performs an FFT. In Fig. 3.26, this effective channel $\mathbf{H}$ is denoted by the dashed box. In this section perfect knowledge of CSI at the receiver side is also assumed. However, a practical receiver implementation estimates the channel response from each transmit antenna with known orthogonal pilot signals sent multiplexed with the data [101]. Therefore, the receiver needs to estimate four and eight channel responses for the $2 \times 2$ and $4 \times 2$ schemes, respectively ${ }^{3}$. The two

\footnotetext{
${ }^{3}$ Compared with SISO, the amount of pilot information has to be doubled and quadrupled for $2 \times 2$ and $4 \times 2$ MIMO schemes, respectively. This amount of pilot information reduces
} 
received streams are then frequency, time and cell de-interleaved to undo the transmitter operations and fed to the MIMO demodulator which provides soft information about the transmitted code bits. It is noted that in the case of two transmit antennas with eSM-PH, the MIMO demodulator takes into account eSM-PH processing. LLRs for the transmitted code bits are calculated using the received data streams and CSI. Next, the LLRs are de-interleaved and processed by the LDPC decoder that runs several iterations of the sum-product algorithm before outputting its decisions to the $\mathrm{BCH}$ decoder.

\subsubsection{Design of MIMO-Channel-Precoders}

\section{MIMO-Channel-Precoder Based on Mean-Optimality}

Now, a channel-precoder is derived with near-optimal performance in the considered broadcast TV channel. This method is based on averaging per-channelrealization optimal covariance matrices. First, slightly abusing terminology, let $\tilde{\mathbf{H}}$ be a possible channel realization. For this specific channel realization, the solution $\tilde{\mathbf{U}}$ matrix is given by the eigenvector matrix of $\tilde{\mathbf{H}}^{\dagger} \tilde{\mathbf{H}}$ and the solution $\tilde{\boldsymbol{\Lambda}}$ matrix is given by the following water-filling solution:

$$
\tilde{\lambda}_{k}=\max \left(\mu-\frac{\sigma^{2}}{\tilde{d}_{k}}, 0\right), \quad k=1,2, \ldots, N_{t},
$$

where $\tilde{\lambda}_{k}$ is the $k^{\text {th }}$ diagonal entry of $\tilde{\boldsymbol{\Lambda}}, \tilde{d}_{k}$ is $k^{\text {th }}$ eigenvalue of $\tilde{\mathbf{H}}^{\dagger} \tilde{\mathbf{H}}, \sigma^{2}$ is the noise power, and the water-filling parameter $\mu$ is chosen such that $\tilde{\lambda}_{1}+\tilde{\lambda}_{2}+\ldots+$ $\tilde{\lambda}_{N_{t}}=N_{t}$. The mean-optimal covariance matrix is then obtained by averaging along all per-channel optimal covariance matrices:

$$
\mathbf{Q}_{\mathrm{MO}}=E\left\{\tilde{\mathbf{U}} \tilde{\Lambda} \tilde{\mathbf{U}}^{\dagger}\right\}
$$

where the statistical expectation is over all possible channel realizations. The resulting MIMO-channel-precoder for the mean-optimal solution is given by

$$
\Gamma_{\mathrm{MO}}=\mathbf{U}_{\mathrm{MO}} \boldsymbol{\Lambda}_{\mathrm{MO}}^{\frac{1}{2}}
$$

where $\mathbf{U}_{\mathrm{MO}}$ and $\boldsymbol{\Lambda}_{\mathrm{MO}}$ are the eigenvector and eigenvalue matrices, respectively, of the mean-optimal covariance matrix $\mathbf{Q}_{\mathrm{MO}}$. The proposed algorithm has low computational complexity and it is a simple tool to optimize the performance of

significantly the available spectral efficiency in mobile scenarios since denser patterns are needed to sample the time-variant channel, e.g., $8,3 \%$ and $16,6 \%$ of pilots assumed for SISO and MIMO $2 \times 2$ in DVB-NGH, respectively. This situation improves in static/portable reception (as the one studied in this section) where sparser pilot patterns can be supported due to time-invariability of the channel e.g., $1 \%$ for SISO DVB-T2 UK mode, $2 \%$ for $2 \times 2$ MIMO, and $4 \%$ for $4 \times 2$ MIMO. 

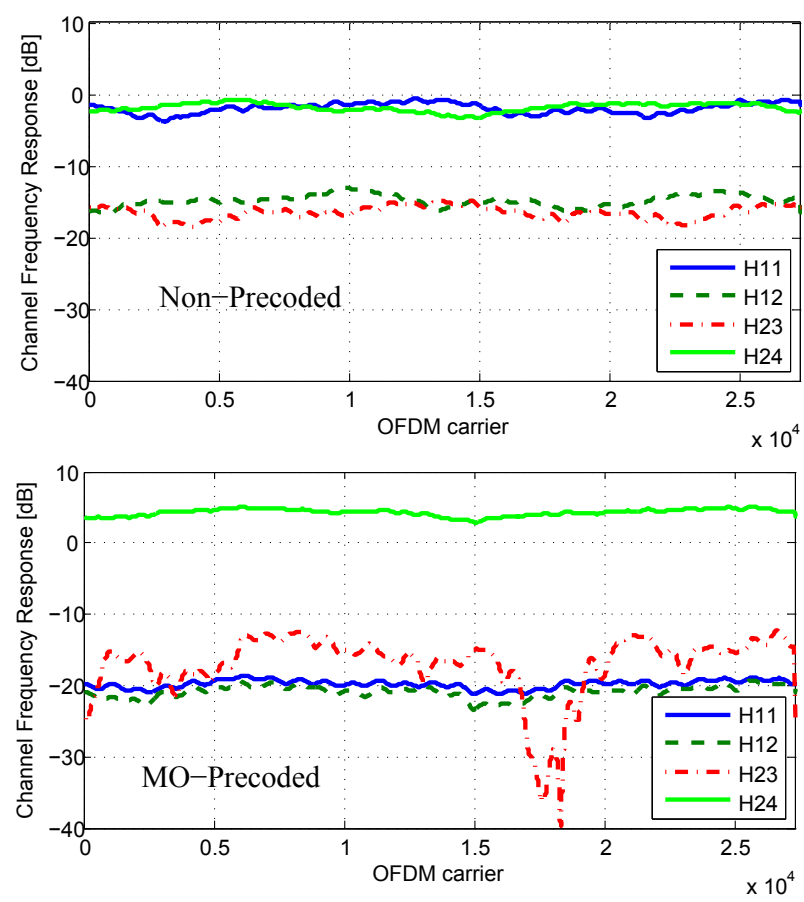

Figure 3.27: Channel frequency responses of a MIMO $4 \times 2$ without precoding (top) and with precoding (bottom) in the MGM channel model.

(c) 2015 IEEE.

generic MIMO channels which exhibit any kind of correlation between antennas and/or LoS condition.

Fig. 3.27 shows a sample channel frequency responses of a MIMO $4 \times 2$ without (top) and with precoding (bottom) under the MGM channel when the precoding is placed after pilot insertion as in Fig. 3.2(b). The precoder does not affect significantly the selectivity of the channel response but modifies the mean power of the effective received channels.

\section{MIMO-Channel-Precoder Based on Jensen's Inequality}

For comparison and completeness, a MIMO precoder based on Jensen's inequality [102] is considered, which was previously used for precoder designs in cellular systems with feedbacks [50]. In this design, instead of maximizing the ergodic capacity expression in (3.10), a tractable upperbound obtained through 
the following derivation is maximized:

$$
\begin{aligned}
& E\left\{\log _{2} \operatorname{det}\left(\mathbf{I}_{N_{r}}+\frac{\rho}{N_{t}} \mathbf{H Q H}^{\dagger}\right)\right\} \\
= & E\left\{\log _{2} \operatorname{det}\left(\mathbf{I}_{N_{t}}+\frac{\rho}{N_{t}} \mathbf{H}^{\dagger} \mathbf{H Q}\right)\right\} \\
\leq & \log _{2} \operatorname{det}\left(\mathbf{I}_{N_{t}}+\frac{\rho}{N_{t}} E\left\{\mathbf{H}^{\dagger} \mathbf{H}\right\} \mathbf{Q}\right),
\end{aligned}
$$

where (3.17) is due to $\log$-determinant identity, $\log \operatorname{det}(\mathbf{I}+\mathbf{A B})=\log \operatorname{det}(\mathbf{I}+$ BA), and (3.18) follows from the Jensen's inequality and the concavity of the log-determinant function over positive semi-definite matrices. Optimizing (3.18) can be done through well known waterfilling algorithm [102]. Consequently, the solution $\mathbf{U}_{\mathrm{J}}$ matrix is given by the eigenvector matrix of $E\left\{\mathbf{H}^{\dagger} \mathbf{H}\right\}$ and the solution $\Lambda_{\mathrm{J}}$ matrix is given by the water-filling solution:

$$
\lambda_{k}=\max \left(\mu-\frac{\sigma^{2}}{\alpha_{k}}, 0\right), \quad k=1,2, \ldots, N_{t},
$$

where $\lambda_{k}$ is $k^{\text {th }}$ diagonal entry of $\Lambda_{\mathrm{J}}, \alpha_{k}$ is the $k^{\text {th }}$ eigenvalue of $E\left\{\mathbf{H}^{\dagger} \mathbf{H}\right\}, \sigma^{2}$ is the noise power, and water-filling parameter $\mu$ is chosen such that $\lambda_{1}+\lambda_{2}+$ $\ldots+\lambda_{N_{t}}=N_{t}$. Finally, the MIMO-channel-precoder solution based on Jensen's inequality is given by:

$$
\Gamma_{\mathrm{J}}=\mathbf{U}_{\mathrm{J}} \boldsymbol{\Lambda}_{\mathrm{J}}^{\frac{1}{2}}
$$

This precoding maximizes (3.18) instead of the ergodic capacity, and consequently leads to a tractable lowerbound to the true channel-precoding capacity.

Channel-precoders in (3.10), (3.16), and (3.20) improve performance of the transmission in ergodic sense. In the broadcasting set-up the multiple receiving users can suffer different propagation conditions. Therefore, in the next sections the performance (gains and degradations) of the channel-precoders is evaluated for various channel environments and channel-precoder miss-matched condition, i.e., channel statistics differ from the ones used to optimized the channel-precoders.

\subsubsection{Capacity Analysis}

Fig. 3.28 shows the ergodic capacity in bpc vs. CNR in $\mathrm{dB}$ for $4 \times 2 \mathrm{MIMO}$ system with no precoding, Jensen-precoder and the MO (Mean-Optimality) precoder under MGM (a) and NGH-PO (b) channels. Channel-precoding is optimized for this specific channel statistics with full LoS correlation, i.e., $\gamma=1$. It can be observed that, compared to the unprecoded $4 \times 2 \mathrm{MIMO}$, the MOprecoder $4 \times 2$ MIMO provides an extra $1.6 \mathrm{bpc}$ under MGM channel, and an 
extra $0.7 \mathrm{bpc}$ uder NGH-PO channel at $25 \mathrm{~dB}$ of CNR. On the other hand, while the channel-precoder solution based on Jensen's inequality outperforms unprecoded system and MO-precoder at low CNRs, it converges to unprecoded system at high CNRs.

Results in Fig. 3.29 present $2 \times 2$ MIMO performance where the use of Jensen and MO precoders show no enhancement at all CNRs. This is due to the low correlation of the MIMO paths in the $2 \times 2$ case. More generally, the performance of channel-precoding in MIMO systems with the same number of transmit and receive antennas converges to an unprecoded system as the CNR increases [50].

Fig. 3.30 presents the ergodic capacity in bpc for unprecoded and precoded $4 \times 2$ MIMO system against the LoS correlation parameter $\gamma$ under MGM channel (left) and the NGH-PO channels (right). Here, the channel-precoders are designed for every $\gamma$ value and target CNR of $0,10,20$ and $30 \mathrm{~dB}$. Therefore, Fig. 3.30 analyzes the performance when the channel statistics match the channel-precoder. Here, for both channels and CNRs, the channel-precoding gain over unprecoded system increases with increasing $\gamma$ factor and furthermore, higher gains are achieved for the MGM channel. Note that the ergodic capacity with channel-precoding converges to unprecoded system at $\gamma=0$, i.e., no LoS correlation between the two $2 \times 2$ MIMO channels. At low CNRs, Jensen precoder has the best performance but converges to an unprecoded system as as the CNR increases. On the other hand, the MO-precoder outperforms unprecoded system for medium to high $\gamma$ values and for all studied CNRs. It is worth noting that higher ergodic capacity can be achieved in a system with channel-precoding and correlated LoS than in an unprecoded system with uncorrelated LoS. Similar conclusion can be extracted from reference [103] for a $4 \times 2$ MIMO system.

Next, Fig. 3.31 presents ergodic capacity in bpc vs. the Riciean $K$ factor of the MGM channel with $4 \times 2$ MIMO system and CNR values of $0,5,20$ and $30 \mathrm{~dB}$. Three values of LoS correlation $\gamma$ are studied, $\gamma=0$ (no correlation), $\gamma=0.8$ (medium to strong correlation) and $\gamma=1$ (full correlation). The performance of the channel-precoders is studied in mismatched condition, i.e., the channel statistics differ from the ones used to design the precoders. In the case of $\gamma=0$, channel-precoders have the same performance to unprecoded system at all studied CNRs and $K$ values. For the other two $\gamma$ cases, the ergodic capacity of channel-precoding increases with increasing $K$ factor. As observed in Fig. 3.28(a), Jensen precoder outperforms MO precoder at low CNRs, while MO-precoder outperforms Jensen precoder at higher CNRs. In this mismatched analysis we can observe that channel-precoders still provide better performance than unprecoded system even in the event of mismatched $K$. Note that in the extreme case of $K=0$, the channel-precoders still provide an improvement. This is is because, even though there is no LoS component 


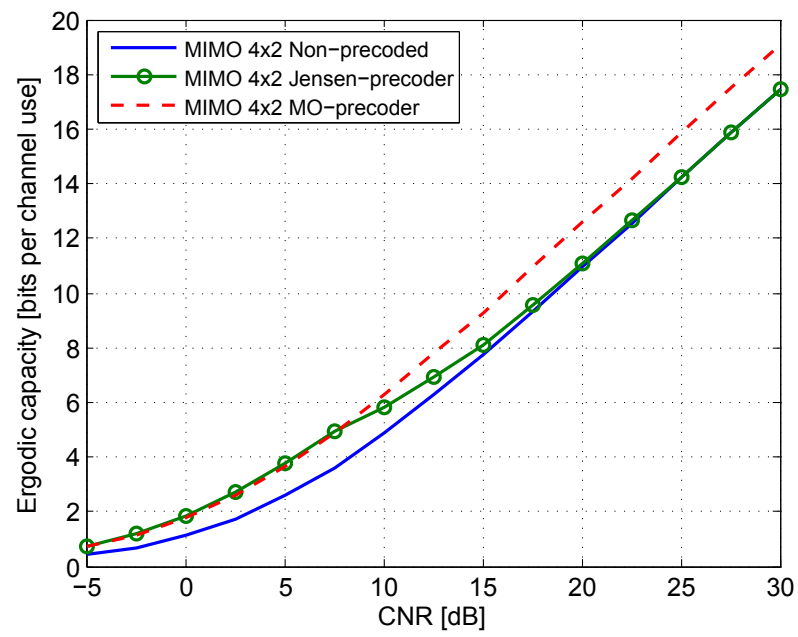

(a) MGM channel model.

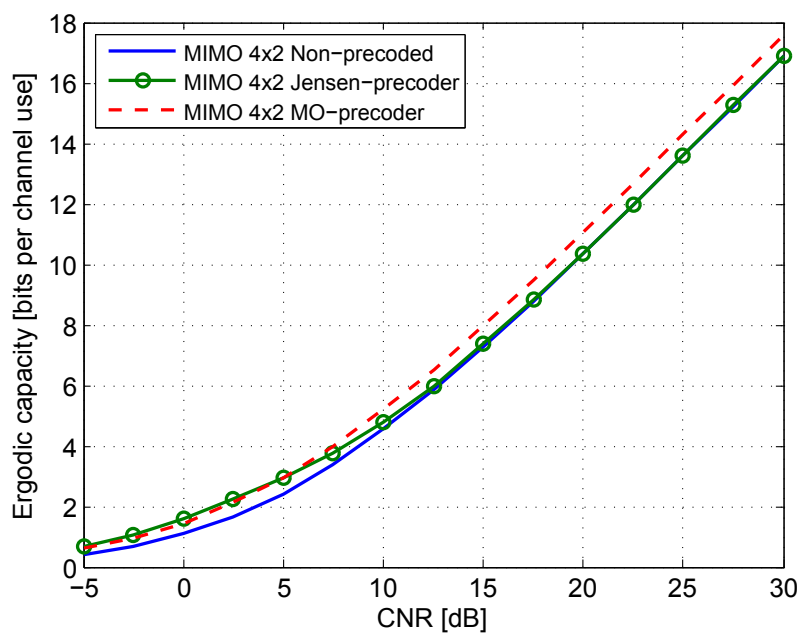

(b) NGH-PO channel model.

Figure 3.28: Ergodic capacity in bpc vs. CNR in dB for MGM (a) and NGH-PO (b) channels with $4 \times 2$ MIMO and LoS correlation $\gamma=1$. Unprecoded system, precoded MIMO with Jensen and MO precoders are illustrated. (Note that in this case of full LoS correlation, the precoding gains are higher for the MGM channel model.) (c) 2015 IEEE. 


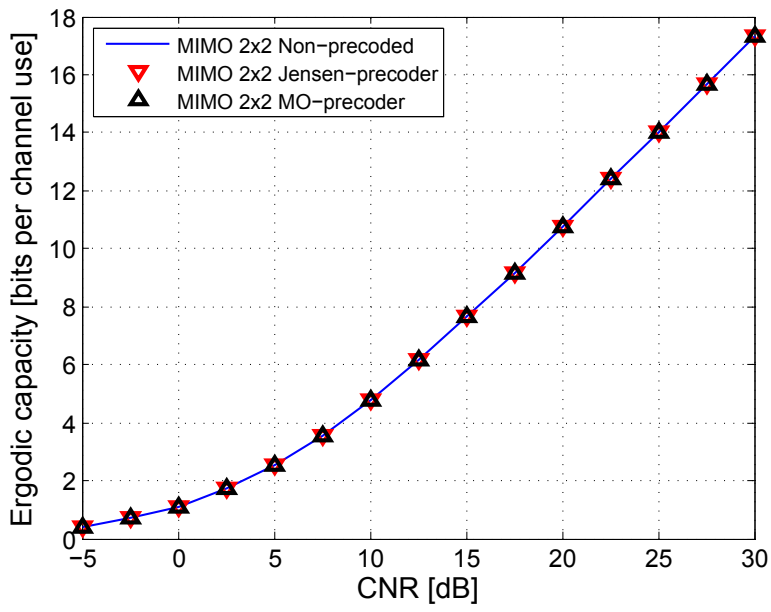

(a) MGM channel model.

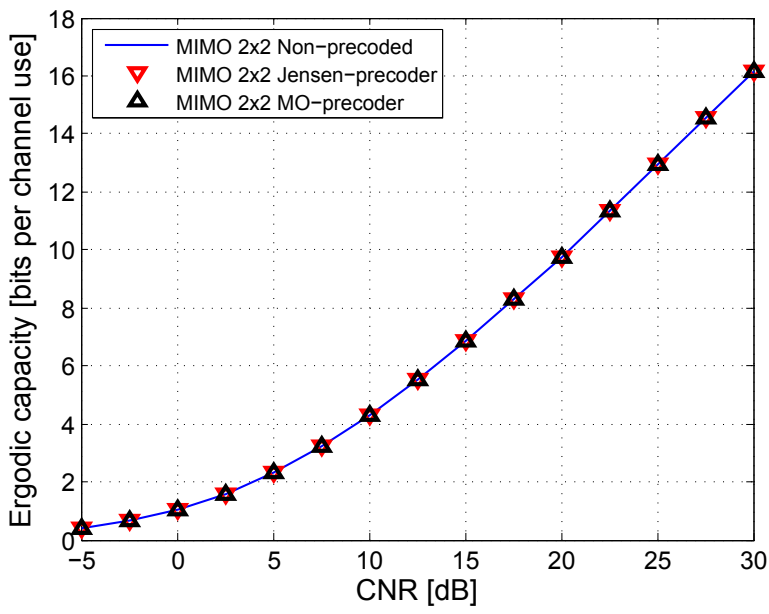

(b) NGH-PO channel model.

Figure 3.29: Ergodic capacity in bpc vs. CNR in dB for MGM (a) and NGH-PO (b) channels with $2 \times 2$ MIMO. Unprecoded system, precoded MIMO with Jensen and MO precoders are illustrated. (C) 2015 IEEE. 

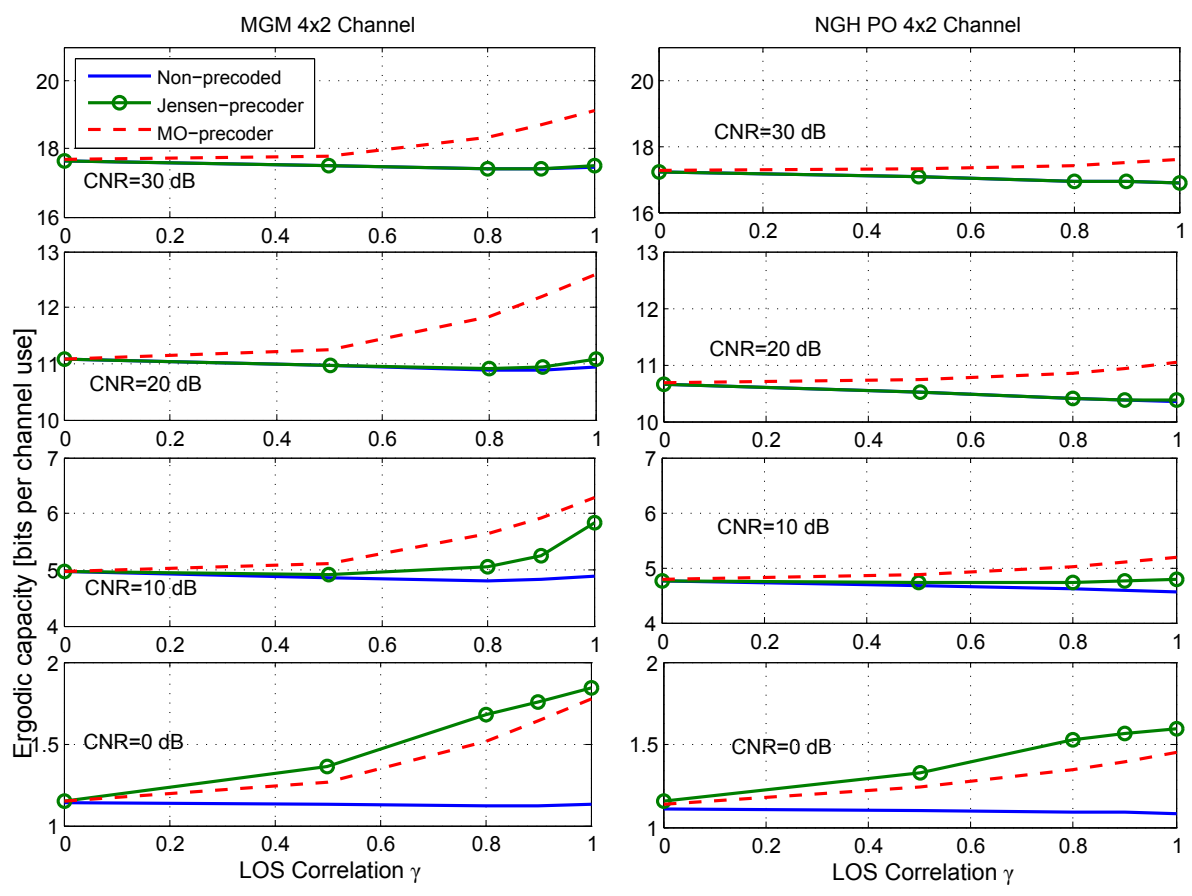

Figure 3.30: Ergodic capacity in bpc vs. LoS correlation $\gamma$ with $4 \times 2$ MIMO for MGM (left) and NGH-PO (right) channels and CNR values of 0, 10, 20 and $30 \mathrm{~dB}$. Unprecoded system, precoded MIMO with Jensen and MO precoders are illustrated. Channel-precoders are designed for every case of LoS correlation $\gamma$ and target CNR (matched case with channel statistics).

(c) 2015 IEEE. 

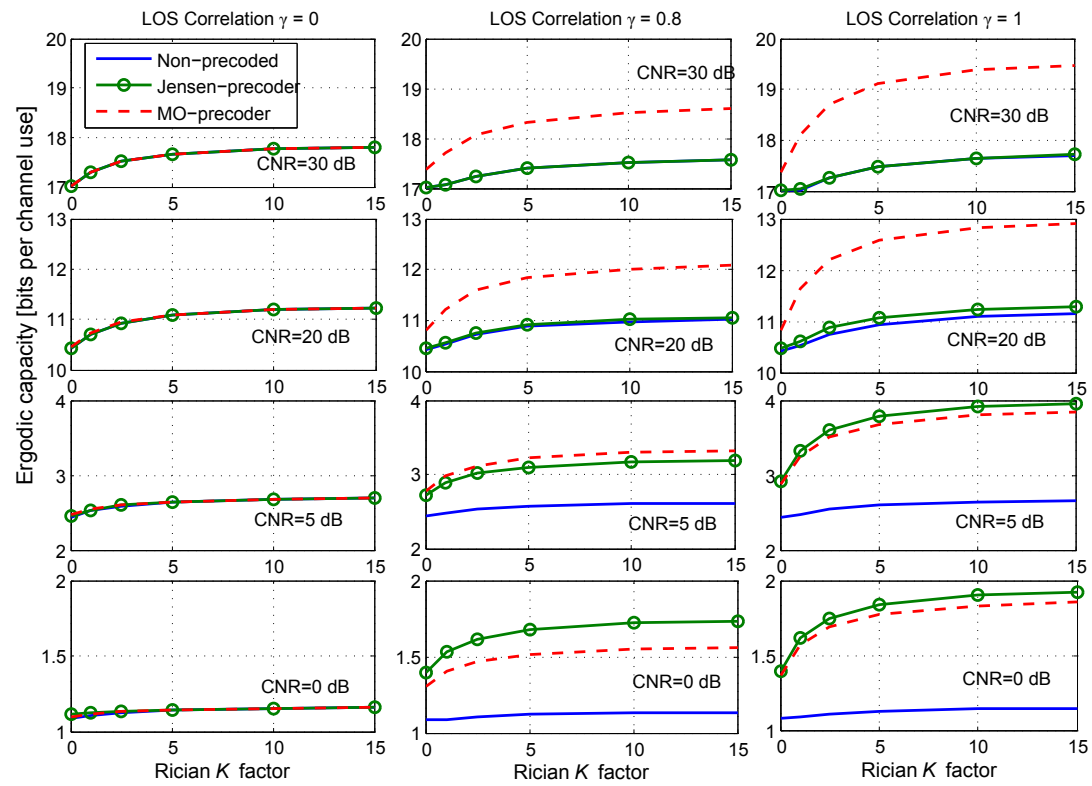

Figure 3.31: Ergodic capacity in bpc vs. Riciean $K$ factor with $4 \times 2 \mathrm{MIMO}$ under MGM channels and CNR values of $0,5,20$ and $30 \mathrm{~dB}$. Three values of LoS correlation $\gamma$ are studied, $\gamma=0, \gamma=0.8$ and $\gamma=1$. Unprecoded system, precoded MIMO with Jensen and MO precoders are illustrated. Jensen and

MO precoders are designed for every target $\mathrm{CNR}$ and fixed $K=5$ (MGM parameter) - mismatched case with the true channel statistics. (c) 2015 IEEE.

in the channel, the channel-precoders are able to exploit the correlation of the Non Line Of Sight (NLoS) component.

\subsubsection{System Performance}

To complement the channel capacity results presented in the previous subsections, BER performance of the considered MIMO systems is simulated.

The MGM rooftop and NGH-PO MIMO cross-polar channels used in this subsection have the values of $K$ and $X$ as defined in Appendix A. Further simulation parameters are specified in Table 3.4, where the precoded MIMO systems used the designed MO-precoder with fixed channel paramters (fixed $K$, $X$ and LoS correlation $\gamma$ factors). Perfect CSI at the receiver side is assumed. The code-rates 5/15,8/15 and 11/15 are selected to evaluate the performance of the different schemes at low, mid and high code-rates. Additionally, on each transmit antenna a 256QAM constellation for SISO, 16QAM constellation for 
Table 3.4: Simulation Parameters.

\begin{tabular}{|c|c|}
\hline System Parameters & Value \\
\hline FFT size & $32 \mathrm{~K}$ \\
\hline Guard interval & $1 / 128$ \\
\hline LDPC block length & 16200 bits \\
\hline Code rate & $5 / 15,8 / 15$, and $11 / 15$ \\
\hline & $256 \mathrm{QAM}-$ SISO \\
Constellation & QPAM - MIMO $2 \times 2$ \\
& Gray labelling \\
\hline Mapping & perfect receive CSI \\
\hline Channel estimation
\end{tabular}

$2 \times 2$ MIMO, and QPSK constellation for $4 \times 2$ MIMO it is used. In particular, 8 bits are transmitted per channel use for all antenna configurations with an effective rate of $2.58,4.18$ and $5.78 \mathrm{bpc}$, respectively when taking into account error control coding ${ }^{4}$.

First, Fig. 3.32 and Fig. 3.33 compare the performance of SISO, MIMO SM (unprecoded) $2 \times 2$, MIMO eSM-PH (NGH precoding) $2 \times 2$ and unprecoded $4 \times 2$ MIMO with LDPC code rates of 5/15 (left), 8/15 (center) and 11/15 (right) under NGH-PO (Fig. 3.32) and MGM (Fig. 3.33) channels. For the unprecoded MIMO SM $4 \times 2$ case, both channels have zero LoS correlation $(\gamma=0)$. For both channels, MIMO schemes show a significant gain compared to SISO. Applying NGH precoding to MIMO $2 \times 2$ provides an advantage over the unprecoded case in the NGH-PO channel (since NGH precoding was optimized for this channel), but it does not for the MGM channel. It is interesting to note that while MIMO NGH $2 \times 2$ provides better or similar performance to unprecoded $4 \times 2$ MIMO in the NGH-PO channel, for the MGM channel MIMO $4 \times 2$ outperforms MIMO NGH $2 \times 2$.

\footnotetext{
${ }^{4}$ This spectral efficiency does not take into account the loss due to signalling, synchronization, pilot insertion, and guard interval.
} 
CHAPTER 3. MIMO PRECODING FOR TERRESTRIAL BROADCAST SYSTEMS

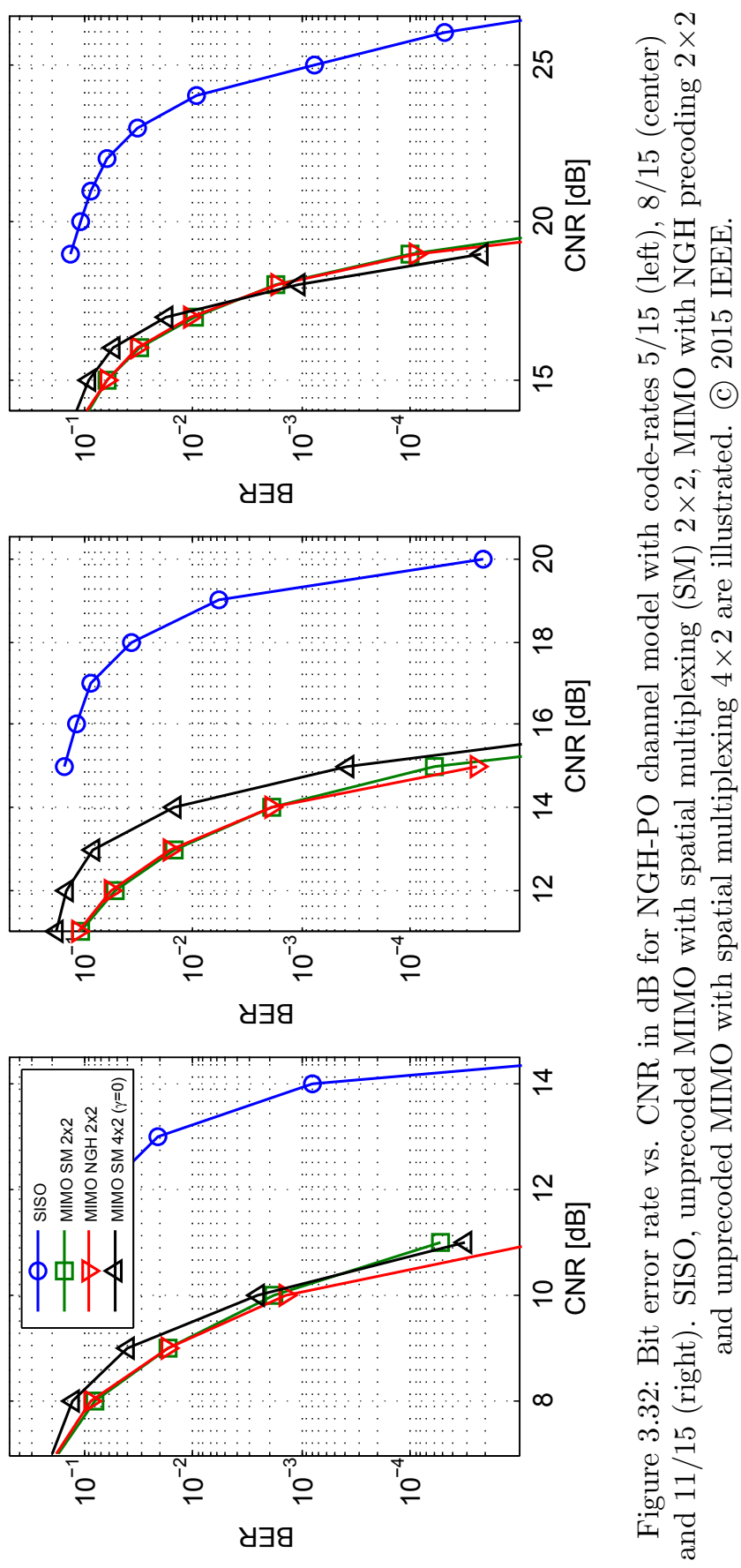



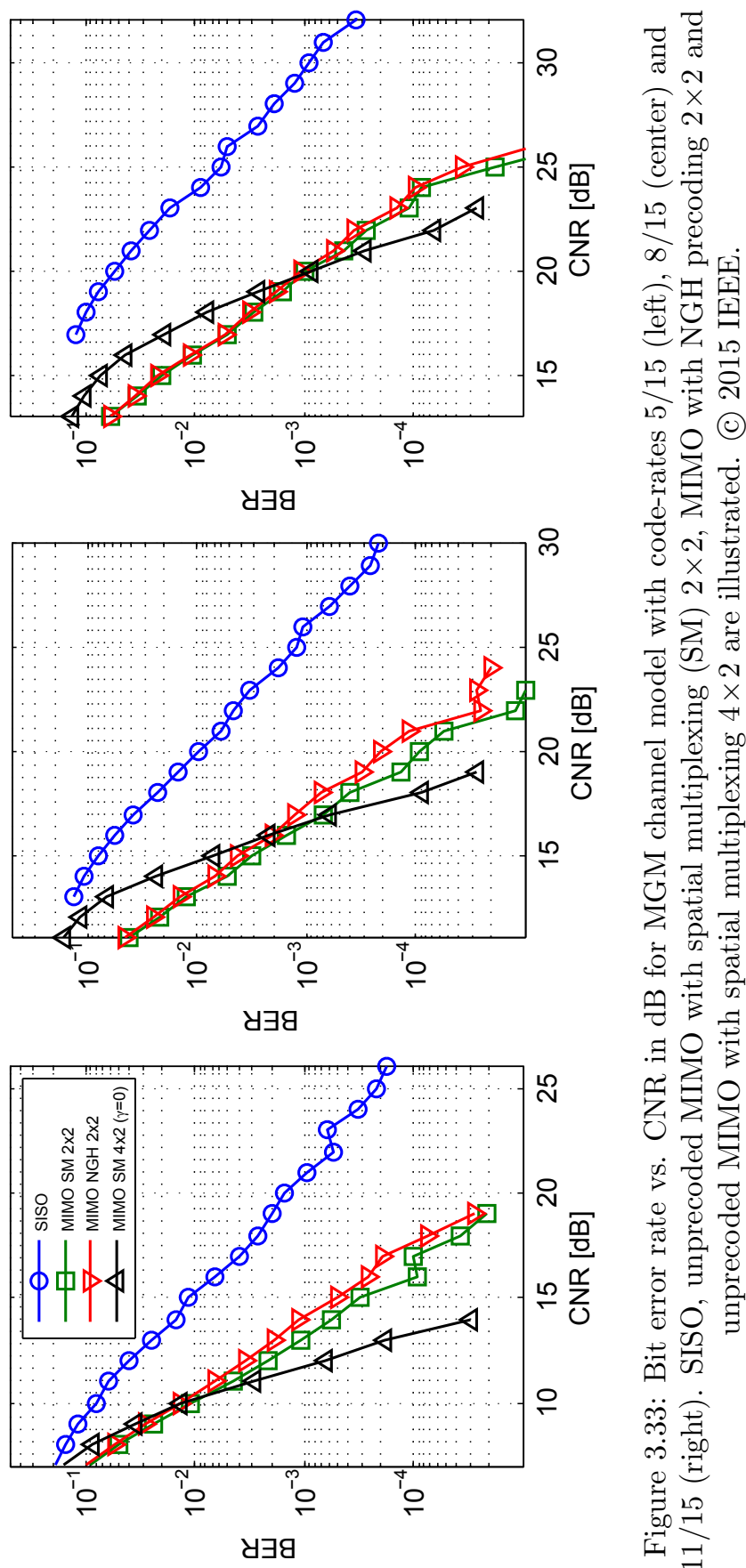
Here, Fig. 3.34 investigates the unprecoded $4 \times 2$ MIMO performance degradation due to LoS correlation under MGM (top) and NGH-PO (bottom) channels . One can observe that the performance degrades with increasing $\gamma$ factor for both channels. However, this degradation is higher in the MGM channel.

Fig. 3.35 and Fig. 3.36 compares the performance of $4 \times 2$ MIMO with the MO-precoder and the unprecoded case in the NGH-PO and MGM channels, respectively. LoS correlation values $\gamma=0.8$ and $\gamma=1.0$ are included. In both channels one can observe that MO-precoding provides improved or similar performance to unprecoded system at code rate $5 / 15$ but incurs in an increasing performance degradation with increasing code rate.

To explain the performance dependence with the code rate of the MOprecoding, Fig. 3.37 presents the pdf of the LLR values at the output of the MIMO demodulator for a MIMO $4 \times 2$ with and without MO-precoding under the MGM channel model with $15 \mathrm{~dB}$ (top) and $25 \mathrm{~dB}$ (bottom). First, it can be observed that MO-precoding affects the distribution of LLR values. With precoding, the LLRs take, with high probability, either small (i.e., low bit reliability) or high absolute values (i.e., high bit reliability). Without precoding the LLR values are more uniformly distributed. The strong reliability for some of the LLR values with precoding can be connected with the improved performance at low code-rates. When channel coding is used, previous works in $[104,63]$ have shown that while diversity techniques improve performance at high code-rates, they can degrade the performance at low code-rates. MO-precoding reduces the diversity of the LLR values in favour of enhancing the reliability of some of the transmitted bits, which can be exploited by the diversity of the channel code at low code-rates.

Finally, in Fig. 3.38 the performance of MO-precoder $4 \times 2$ MIMO is analysed in the case of mismatch condition with a $K$ factor, where the precoder statistics and true channel statistics differ. This is common situation in the broadcasting set-up, since different users can experience channels with different reception conditions and therefore different channel statistics. Here, the gain is compared over unprecoded $4 \times 2 \mathrm{MIMO}$ in the NGH-PO channel with two values of $\gamma$ equal to 0.8 and 1.0. The performance of code rate $5 / 15$ is studied since higher ones provided poor performance for channel-precoding. The gain increases for both values of $\gamma$ with increasing $K$ factor. It is interesting to note that for this low code-rate even in the extreme case of $K=0$ (where there is no LoS component) the channel-precoder still provides a gain of about $0.5 \mathrm{~dB}$. This is because the precoder is still able to exploit the covariance matrix of the NLoS components which also has some degree of correlation (cf. Appendix A). 


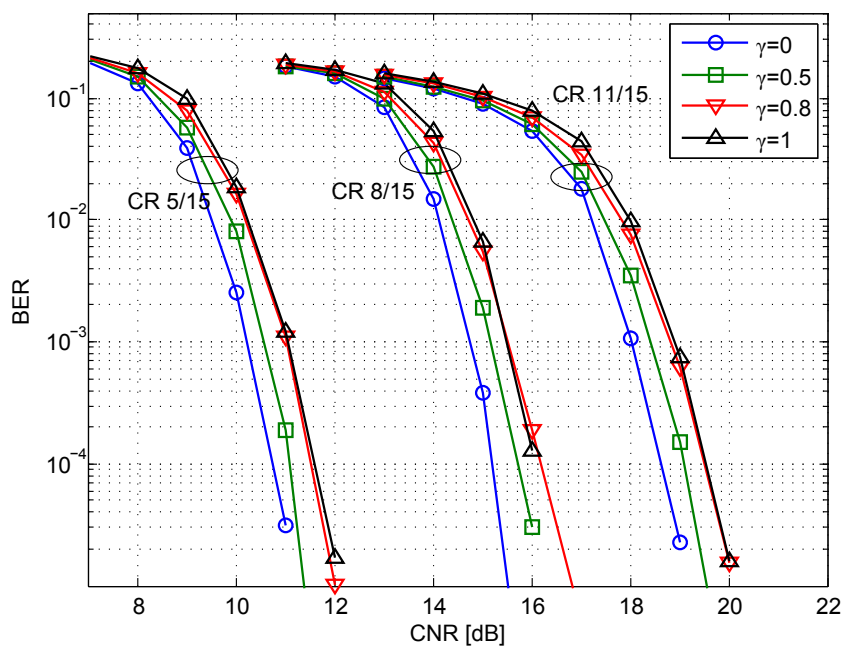

(a) NGH-PO channel model.

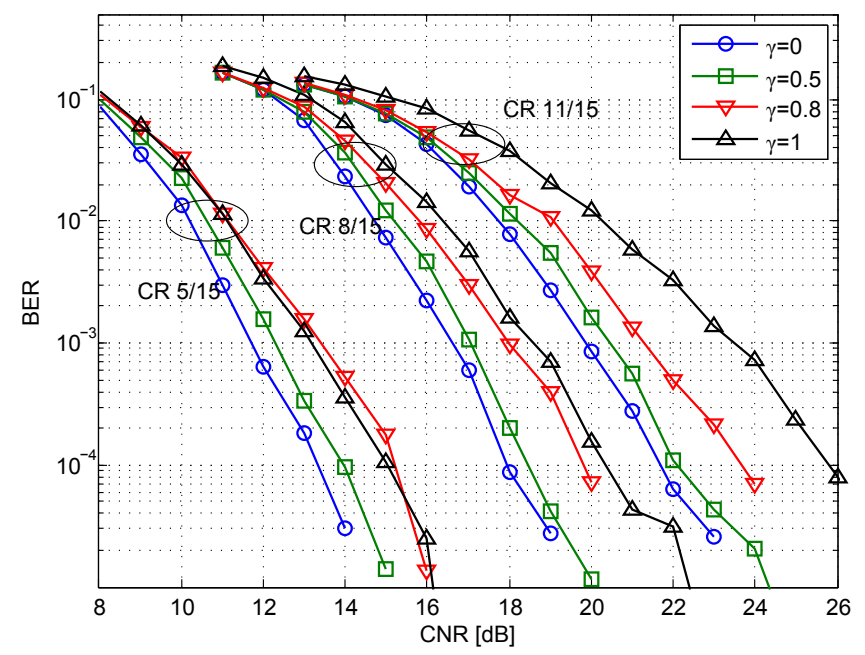

(b) MGM channel model.

Figure 3.34: Bit error rate vs. CNR for NGH-PO (upper) and MGM (bottom) channel models with code-rates 5/15, 8/15 and 11/15. Unprecoded MIMO with spatial multiplexing $4 \times 2$ with different LoS correlation $\gamma$ values is illustrated. (c) 2015 IEEE. 


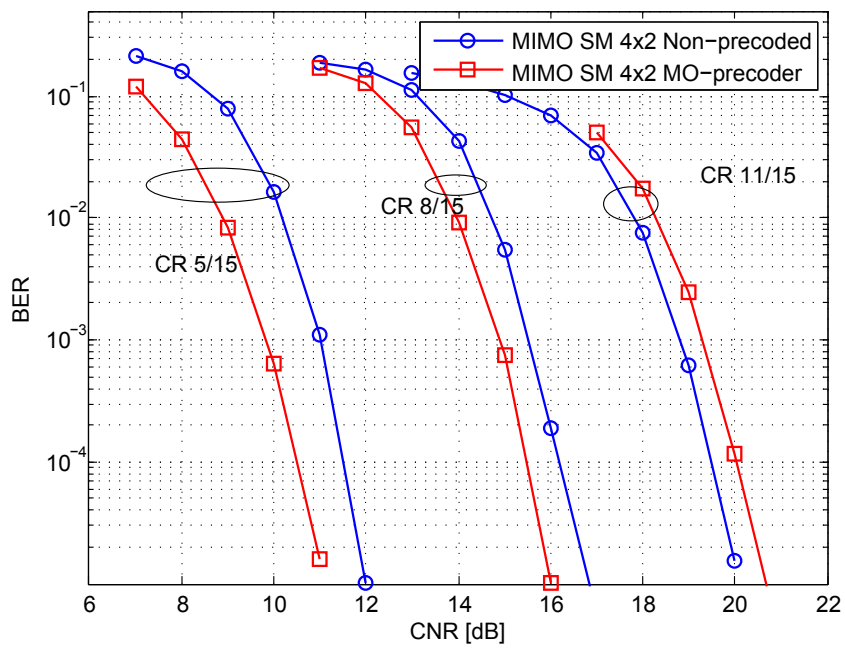

(a) LoS correlation $\gamma=0.8$.

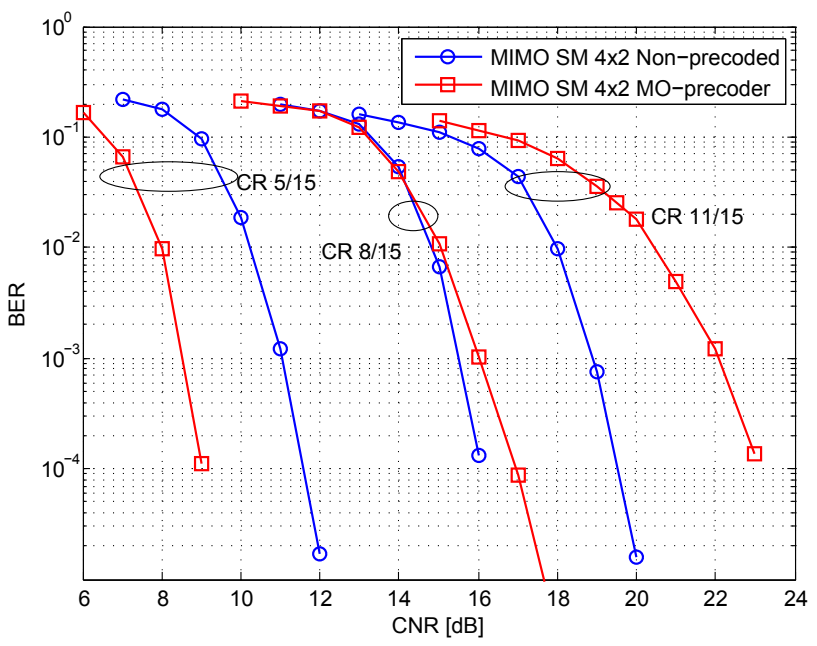

(b) LoS correlation $\gamma=1.0$.

Figure 3.35: Bit error rate vs. CNR in $\mathrm{dB}$ for NGH-PO channel model with code-rates $5 / 15,8 / 15$ and $11 / 15$. MIMO with simple spatial multiplexing $4 \times 2$ and MIMO with simple spatial multiplexing $4 \times 2$ with MO-precoder for $\gamma=0.8$ (upper) and $\gamma=1.0$ (bottom). (c) 2015 IEEE. 


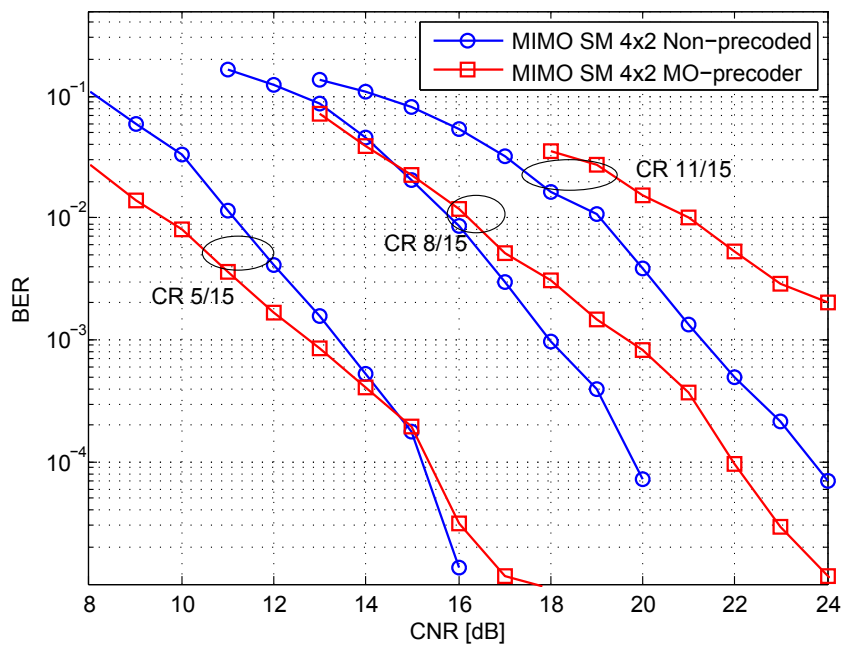

(a) LoS correlation $\gamma=0.8$.

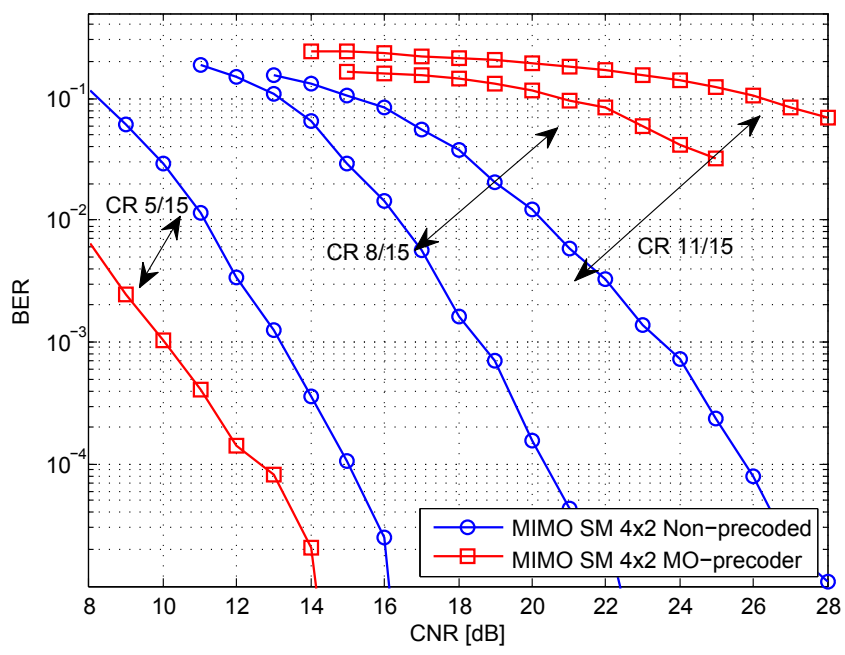

(b) LoS correlation $\gamma=1.0$.

Figure 3.36: Bit error rate vs. CNR in dB for MGM channel model with code-rates 5/15, 8/15 and 11/15. MIMO with simple spatial multiplexing $4 \times 2$ and MIMO with simple spatial multiplexing $4 \times 2$ with MO-precoder for $\gamma=0.8$ (upper) and $\gamma=1.0$ (bottom). (c) 2015 IEEE. 

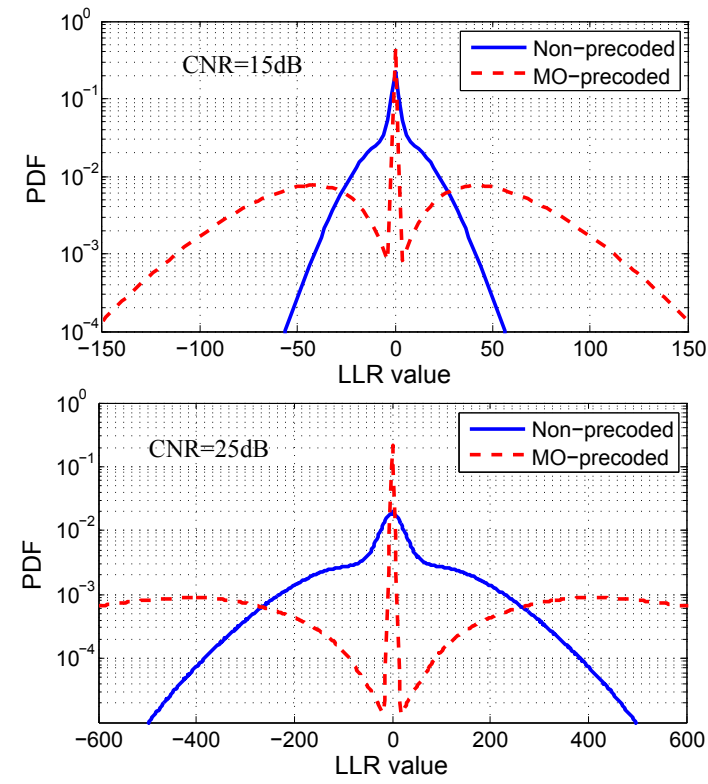

Figure 3.37: Probability density function of the LLR values for MIMO $4 \times 2$ without precoding and with MO-precoding under MGM channel with $15 \mathrm{~dB}$ (upper) and $25 \mathrm{~dB}$ (bottom) of received CNR. (c) 2015 IEEE.

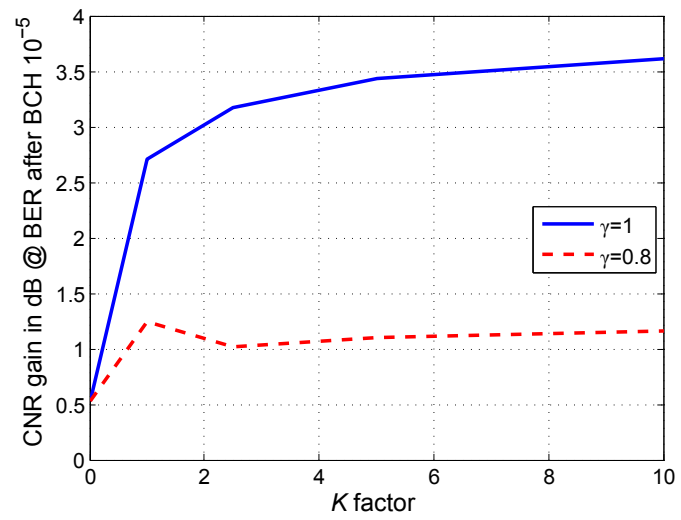

Figure 3.38: CNR gain in dB vs. $K$ factor for MIMO $4 \times 2$ with MO-precoder over MIMO $4 \times 2$ without precoding in NGH-PO channel with code-rate $5 / 15$. MO precoder is designed for fixed $K=5$ (mismatched case). (c) 2015 IEEE. 


\subsection{Summary and Conclusions}

This thesis has investigated and characterized the performance of MIMO precoding in DTT systems. First, the performance and structure of MIMO precoders based on rotation matrices for $2 \times 2 \mathrm{MIMO}$ has been investigated, giving special attention on the case of cross-polar antennas, which is the preferred configuration in DTT systems in the UHF band. This type of precoding has been characterized against: (i) channel parameters such as antenna orientation, spatial correlation, and transmit PI, and (ii) transmission parameters such as constellation order and physical layer FEC code-rate. The results based on Monte-Carlo simulations of the information theoretical limits of MIMO-BICM systems and system BER with practical channel codes show the interesting result that, in general, the optimal rotation angle depends on the physical layer FEC code-rate. In particular with cross-polar antennas, the rotation gain increases for increasing code-rate, i.e., at low code-rates it is optimal not to rotate, and at mid and high code-rates it is optimal to rotate. Then, the performance of the stream-power-allocation matrix with constellations of different cardinality in each transmit antenna has been studied. The evaluation in the case of cross-polar antennas shows that allocation of more power to the constellation with higher cardinality can improve the performance from mid to high code-rates. This can be achieved by either intentional transmit PI between antennas or stream-power allocation. Finally, the precoding solutions adopted in DVB-NGH and ATSC 3.0 have been compared with the results obtained in this chapter. These comparisons show that the adopted parameters for the precoding in DVB-NGH can be suboptimal for some transmission configurations. On the other hand, for ATSC 3.0, a per code-rate rotation angle optimization allows optimal rotation performance at each transmitted spectral efficiency.

Secondly, in this chapter a MIMO-channel-precoding has been proposed that exploits statistical information of the MIMO channel. This precoder has the potential to further increase the channel capacity when compared to equivalent unprecoded MIMO set-up. The performance of the proposed channelprecoder is evaluated for fixed and portable channels and various reception conditions. A mismatched analysis allows to evaluate the performance of the precoder when the channel statistics do not match the precoder, a typical situation in the broadcast set-up. Capacity results present performance enhancements in scenarios with strong LoS and correlated antenna component, and resilience in mismatched condition for the considered $4 \times 2$ MIMO systems, but no enhacement for $2 \times 2 \mathrm{MIMO}$ systems. BER simulation results with the DVB-NGH MIMO physical layer show that for low code rates, performance improvements can be achieved in the case of strong LoS correlation and resilience against mismatched condition with the channel statistics. 
CHAPTER 3. MIMO PRECODING FOR TERRESTRIAL BROADCAST SYSTEMS 


\section{Chapter 4}

\section{Receiver Memory and Performance Trade-Off of Soft-Quantized Information}

7 HE PREVIOUS CHAPTER assumed perfect reception conditions such as opever, due to complexity constraints and finite-precision arithmetic, it is crucial for the overall system performance to carefully design receiver algorithms. This chapter investigates memory and performance trade-offs of soft-quantized information in MIMO-BICM receivers. DTT systems, such as DVB-NGH, rely on TI techniques to overcome signal fluctuations and improve the system performance by time-diversity gain. Yet, long TI imposes the highest in-chip memory requirements at the receiver, which depends on the quantization resolution and specific algorithms. Two types of quantized receivers are investigated: quantization of I\&Q samples and quantization of log-likelihood ratios. The implications on the in-chip memory and the possibility of implementing MIMO-BICM with iterative decoding are presented and discussed. Additionally, the performance degradations of uniform quantization and non-uniform quantization algorithms is evaluated showing significant potential benefits for non-uniform quantization adapted to the signal statistics. The results obtained in this chapter highlight the important trade-off between in-chip memory and performance for quantized receiver architectures. 


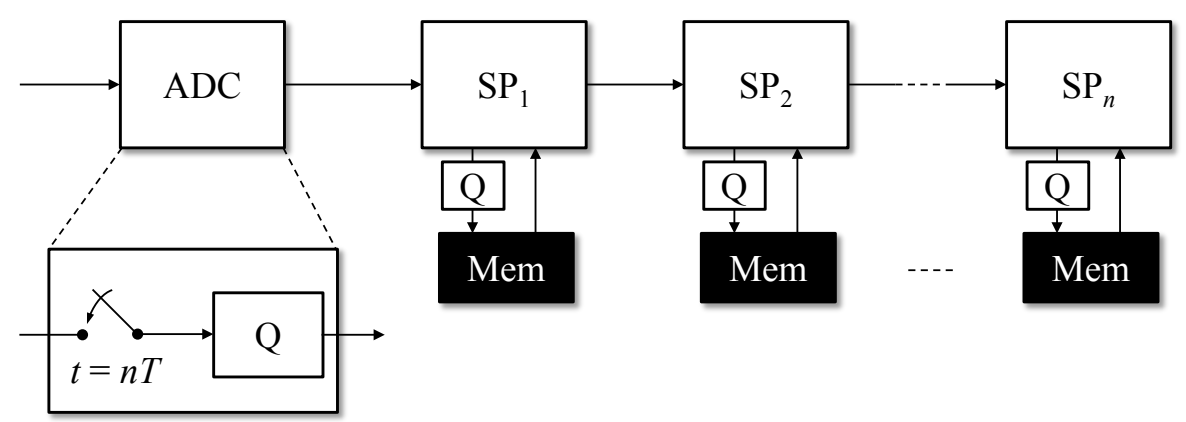

Figure 4.1: Block diagram of quantization stages in a generic receiver.

\subsection{Quantized receiver Architectures}

Signal quantization is a fundamental operation that can be found in any digital receiver. Quantization permits transformation of continuous signals (e.g., sound, light, radio waves, etc.) to a discrete signal sequence (analog-to-digital converters) that can be processed (signal-processing), transmitted and/or stored in conventional digital processors (cf. Fig. 4.1). A low quantization rate is highly beneficial for hardware implementations, since it entails a reduction of chip area, which in turn yields a lower power consumption. However, a fundamental trade-off connects quantizer resolution and distortion. The use of quantizers adapted to the signal statistics can minimize the degradations for a given quantization rate.

Analog-to-Digital Converters (ADC) realize two basic operations, signal sampling and quantization. The ADC first samples the continuous input signal with a sampling rate higher than twice the highest frequency of the input signal to fulfil the Nyquist rate signalling criterion. The quantizer then maps input sampled values to a discrete set of $2^{q}$ values, where $q$ is the number of quantization bits (resolution), while at the same time the goal of the quantizer is to represent the input signal with little distortion as possible. The resolution of the ADCs would depend on the final application, but for communication systems typical $q$ values are between to 8 to 16 bits [105]. In this dissertation it is assumed that quantization noise introduced by the ADCs is low enough so it has a negligible effect in the final receiver performance.

Other signal processing blocks in the receiving chain may require to store a certain amount of samples into in-chip memory prior to the computation of specific algorithms to a block of data (cf. Fig. 4.1). This can be the case as for frequent processing operations in wireless communication receivers which are listed next. 
FFT operation The FFT processing requires the storage of $N$ samples prior to the FFT operation. The value of $N$ depends on the application, but for DTT systems $N$ takes values from $1024(1 \mathrm{~K})$ to 32768 (32K) samples.

Channel estimation Prior to the estimation of the CSI in the data carriers, the receiver requires the storage of pilot information in frequency and time domain in doubly selective channels. For instance, in DTT systems such as DVB-T2, DVB-NGH and ATSC 3.0, pilot signals are sent multiplexed with the data in a comb-type pilot arrangement. In particular, [45] recommends, for a DVB-T2 system, the storage of two and four OFDM symbols to perform temporal interpolation for FFT sizes of $32 \mathrm{~K}$ and $16 \mathrm{~K}$, respectively.

Interleaving stages Frequency, cell, time and bit de-interleaving stages also require the storage of sampled data before the permutation to the block of received data can be applied. Taking as an use case the receiving chain of DVB-NGH, the storage requirements for the different de-interleaving stages are:

- Frequency de-interleaving: one OFDM symbol where the number of samples depend on the FFT size.

- Cell de-interleaving: one FEC code-word, where the number of samples depend on the FEC block size (e.g., 16200 or 64800 bits) and the cardinality of the constellation, e.g., 4050 samples with 16200 bits FEC code-word size and $4 \mathrm{bpc}$.

- Bit de-interleaving: one FEC code-word, where the number of samples depend on the FEC block size, e.g., 16200 or 64800 bits.

- Time de-interleaving: multiple FEC code-words where the number of samples depend on the TI depth at the transmitter and the antenna configuration. For instance, in the SISO profile of DVB-NGH the number of cells to be stored at the receiver are 524288 cells (for the data and CSI paths). Furthermore, the memory requirements can increase for the MIMO case as it will be discussed in this chapter.

It is important to note that the final amount of memory in bits required to store the samples of the previous processing blocks directly depends on the resolution of the quantization algorithms. From the previous requirements, it can be clearly seen that Time de-Interleaving (TDI) in DTT systems is the stage with the highest memory requirements [45]. Hence, the study of inchip memory and performance trade-off of practical receiver architectures with quantized signals is highly motivated. 


\section{CHAPTER 4. RECEIVER MEMORY AND PERFORMANCE TRADE-OFF OF SOFT-QUANTIZED INFORMATION}

Figures 4.2 and 4.3 present simplified transmit to receive communication chains where the receivers implement, at different parts of the receiving chain, signal quantization to store received information at the TDI memory. At the transmitter, first the information bits are encoded by the LDPC and BCH codes. Then, the encoded bits are bit-interleaved $\left(c_{l}\right)$ and fed to the constellation mapper, which outputs constellation symbols. Finally, the output constellation symbols are time-interleaved and transmitted through the channel.

The architecture in Fig. 4.2 first needs to store the received (distorted) constellation symbols and the channel estimates in order to perform TDI. Before storing into the TDI memory, the in-phase (I) and quadrature (Q) components of the received signals $\mathbf{y}$ and the channel estimates $\mathbf{H}$ are quantized. The quantizer uses the algorithms described in section 4.2.1. Prior to the TDI operation, the quantized symbols are stored until the entire TDI memory is filled. Then, the demapper can use the quantized symbols $\mathbf{y}_{\mathrm{q}}$ and quantized channel estimates $\mathbf{H}_{\mathrm{q}}$ to produce LLRs. (In this chapter the MAP demapper with the max-log approximation is employed cf. Chapter 2.) The output LLRs are bit de-interleaved and passed to the LDPC decoder which performs multiple operations of the max-sum algorithm. With iterative MIMO demapping, the soft-ouput information of the LDPC decoder is passed back to the demapper through a bit-interleaving to output refined soft-decisions.

The architecture in Fig. 4.3 realizes the quantization in a different point of the receiver chain. With this architecture the demapping and TDI positions are exchanged, i.e., demapper is executed before the TDI. If the TDI block deinterleaves groups of LLRs corresponding to the received symbols and channel estimates, there is no effect in the performance [52]. It is assumed that the demapper operates with full resolution. This assumption is reasonable since only the received symbols from each antenna (i.e., $y_{1}$ and $y_{2}$ ) and channel estimates $\left(h_{11}, h_{12}, h_{21}\right.$ and $\left.h_{22}\right)$ from a single channel realization need to be stored with full resolution. The ouput LLRs $\left(\Lambda_{l}\right)$ at the demapper are quantized $\left(d_{l}\right)$ prior to storage to the TDI. This approach is also described in [52]. Once the TDI is filled, the groups of LLRs are de-interleaved and passed to the bit deinterleaving which are finally fed to the LDPC decoder. With this architecture, iterative decoding of the MIMO-BICM receiving chain is not possible. This is because for performing iterative decoding of the MIMO-BICM, the receiver needs access to previous instances of $\mathbf{y}$ and $\mathbf{H}$, which are not available with this structure. 


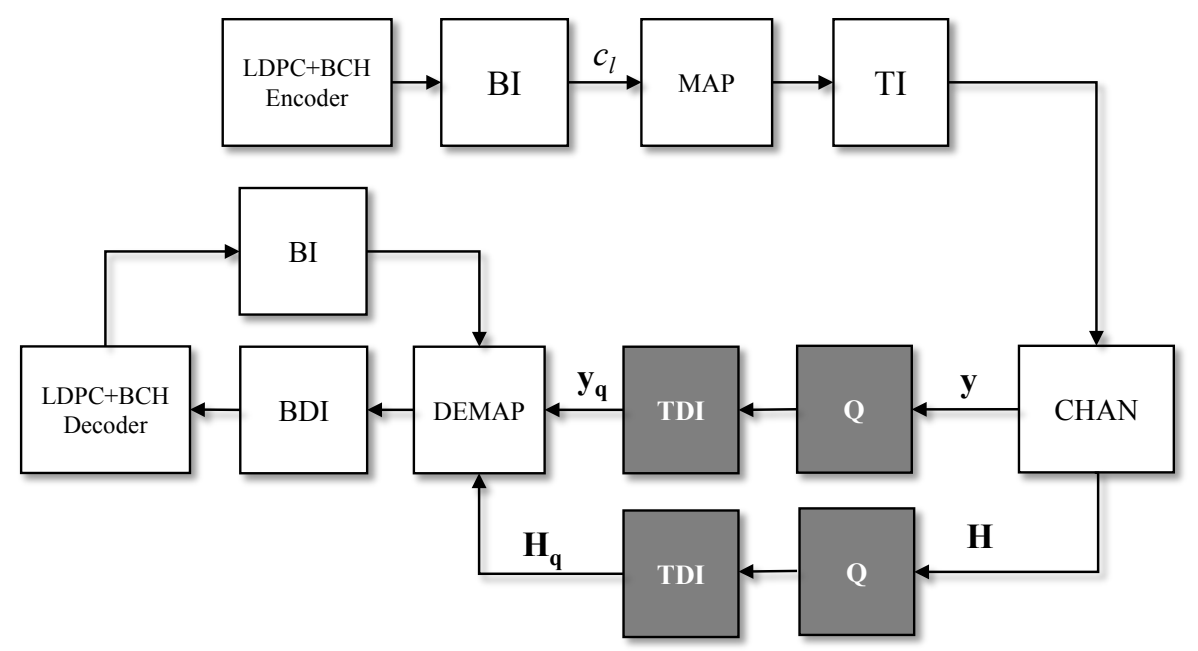

Figure 4.2: Simplified transceiver architecture with quantized In-phase and

Quadrature components of received signals and channel estimates. BI: bit-interleaving, MAP: mapper, TI: Time interleaving, CHAN: channel,

DEMAP: demapper, Q: quantizer, TDI: time de-interleaving, BDI: bit-deinterleaving.

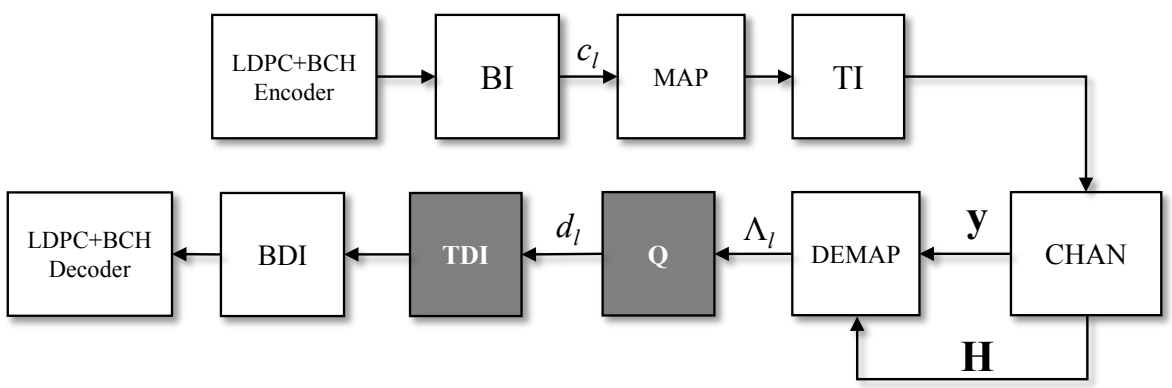

Figure 4.3: Simplified transceiver architecture with quantized log-likelihood ratios. BI: bit-interleaving, MAP: mapper, TI: Time interleaving, CHAN: channel, DEMAP: demapper, Q: quantizer, TDI: time de-interleaving, BDI: bit-deinterleaving. 


\subsection{Quantization Algorithms}

\subsubsection{Quantization of I\&Q Signal Components}

For the receiver structure in Fig. 4.2 the signal components $\mathbf{y}$ and $\mathbf{H}$ need to be quantized. This chapter considers only scalar quantization, hence, the I and $\mathrm{Q}$ components of each of the elements of the vector $\mathbf{y}$ (i.e., $y_{1}$ and $y_{2}$ ) and the matrix $\mathbf{H}$ (i.e., $h_{11}, h_{12}, h_{21}$ and $h_{22}$ ) are quantized independently.

\section{Uniform quantization}

With uniform quantization of I\&Q components it is assumed that the receiver can adapt the maximum quantization range to the received signal. In particular this is done in this chapter by measuring the maximum range of the signal for every received frame. This is clearly an idealization since in a practical receiver design, the signal dynamic range should be decided beforehand to compute a static set of quantizer reproducers and boundaries. This assumption will provide optimistic results for the uniform quantization of I\&Q components. The information of the maximum signal range could be limited in practice by the dynamic range of the $\mathrm{ADC}$ which could be used for the design of the uniform quantizer.

\section{Non-Uniform quantization: Lloyd-Max Algorithm}

For non-uniform quantization of I\&Q components, the Lloyd-Max algorithm [106, 107] is selected and the main steps of the algorithm are presented next.

The Lloyd-Max algorithm finds the quantizer reproducers and boundaries that minimizes the MSE between the input $y$ and the quantized output $z$, i.e., $E\left\{(y-z)^{2}\right\}$. The MSE associated with the quantizer $Q$ can be expressed as:

$$
\mathrm{MSE}=E\left\{(y-z)^{2}\right\}=\int_{\mathcal{Y}}(y-Q(y))^{2} p(y) \mathrm{d} y,
$$

which can expressed with respect to the quantization regions (cf. Chapter 2) as:

$$
\mathrm{MSE}=\sum_{i=1}^{n} \int_{\mathcal{Y}_{i}}\left(y-z_{i}\right)^{2} p(y) \mathrm{d} y,
$$

where $\log _{2} n=q$.

For the optimization of the quantizer boundaries and reproducers with the Lloyd-Max algorithm, two necessary conditions are defined (assuming $n$ reproducer values and known $p(y)$ : 
1. For fixed reproducer values, the quantizer boundary $b_{i}$ between regions $\mathcal{Y}_{i}$ and $\mathcal{Y}_{i+1}$ that minimizes the MSE is the point equidistant to the quantization reproducers $z_{i}$ and $z_{i+1}$, i.e.,

$$
b_{i}=\frac{z_{i}+z_{i+1}}{2}, \quad i=1, \ldots, n-1 .
$$

2. For fixed quantizer boundaries, the optimal reproducers are the centroids at each quantization region:

$$
z_{i}=\frac{\int_{b_{i-1}}^{b_{i}} y p(y) \mathrm{d} y}{\int_{b_{i-1}}^{b_{i}} p(y) \mathrm{d} y}, \quad i=1, \ldots, n .
$$

The Lloyd-Max algorithm starts with an arbitrary initial set of $n$ quantizer reproducers and then executes equations (4.3) and (4.4) until the additional improvement over the MSE minimization is lower than a predefined threshold, or the a maximum number of iterations has been reached.

Figure 4.4 shows the pdf of the I component of the received symbol at the first received antenna for a received $\mathrm{CNR}$ of $11.25 \mathrm{~dB}$. This figure also shows the quantizer reproducers and boundaries with non-uniform distribution using the Lloyd-Max algorithm. As we can observe, a more dense sampling is done at the center of the pdf where it has the highest density. It is interesting to note that the resulting pdf has Gaussian like distribution. It was confirmed with simulations that very similar performance is obtained by using either the measured pdf or a Gaussian approximation for the design of the quantizer with the Lloyd-Max algorithm. In the following evaluations, the measured pdfs in the selected environments are used for the design of the quantizers with the Lloyd-Max algorithm.

\subsubsection{Quantization of Log-likelihood Ratios}

Here, scalar $n$-level quantizers $Q_{l}: \mathbb{R} \mapsto \mathcal{N}_{l}, l=1,2, \ldots, m$ are considered, where $\left|\mathcal{N}_{l}\right|=n=2^{q}$. It is noted that depending on the mapping, the quantizers will in general depend on the code bit position $l$. Specific fixed-point algorithms for the demodulator and the channel decoder are not used. Hence, for a given demodulator the results in this chapter constitute an upper bound on the performance achievable by any implementation under quantized softoutput demodulation. From a system level perspective it would be desirable to have demodulators which directly produce quantized LLRs, instead of computing them at full resolution before quantization. For a specific variant of 


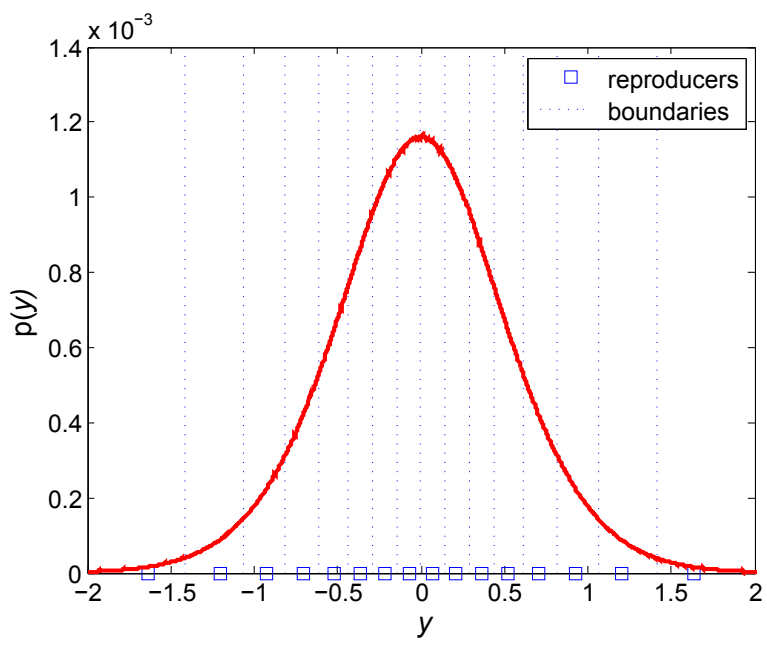

Figure 4.4: Example pdf of In-phase $y$ component for a received CNR of $11.25 \mathrm{~dB}$ with non-uniform quatization reproducers and boundaries designed with Lloyd-Max algorithm.

the sphere decoder [108] and for a linear MMSE receiver [109] there exist such demodulators. However, the development of soft-output demodulators with quantized output is beyond the scope of this thesis.

In what follows, it is assumed that the joint distribution $p\left(\Lambda_{l}, c_{l}\right)$ is known or approximated by measurement. The knowledge of $p\left(\Lambda_{l}, c_{l}\right)$ does not imply perfect CSI, in fact, the quantizer designs used in this chapter are also applicable in the case of imperfect CSI. For the sake of notational simplicity, the code bit index $l$ is omitted in the rest of this section. Depending on the mapping, one needs to design up to $m$ different quantizers. For example, with a square QAM constellation and Gray labeling, there are (at most) $m / 2$ different LLR statistics and, hence, different quantizers.

\section{Uniform quantization}

In this subsection $p\left(\Lambda_{l}, c_{l}\right)$ is quantized using uniform quantizer reproducers and boundaries. Here, although uniform quantization does not require full information about about $p\left(\Lambda_{l}, c_{l}\right)$, information about the dynamic range of the LLRs helps the definition of the first and last quantization reproducers. Fig. 4.5 shows an example of the pdf of the received LLR values with a CNR of $11.25 \mathrm{~dB}$ with 8 bpc for the Most Significant Bit (MSB). Fig. 4.5 also includes quantizer reproducers and boundaries using uniform quantization. The maximum LLR 


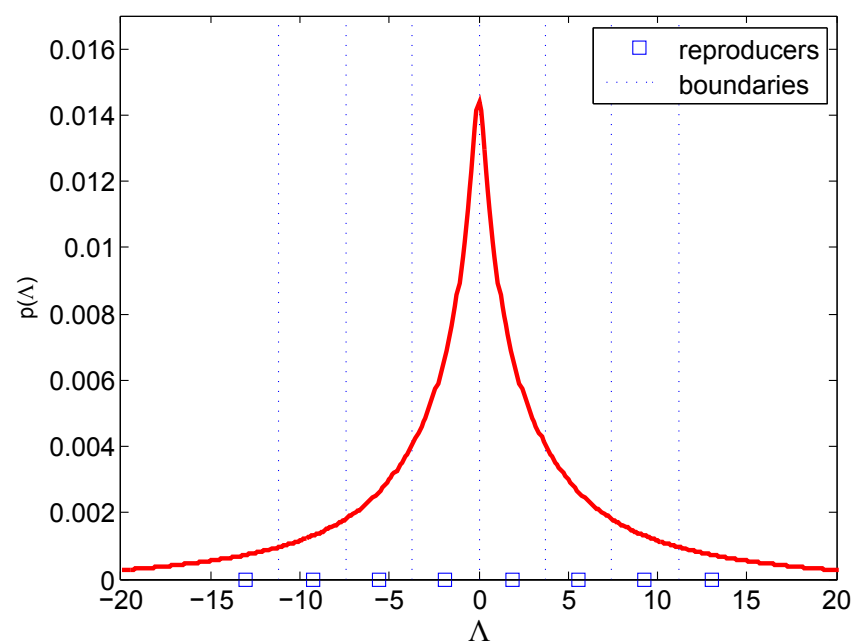

Figure 4.5: Example pdf of $\Lambda$ values for a received CNR of $11.25 \mathrm{~dB}$ with uniform quatization reproducers and boundaries.

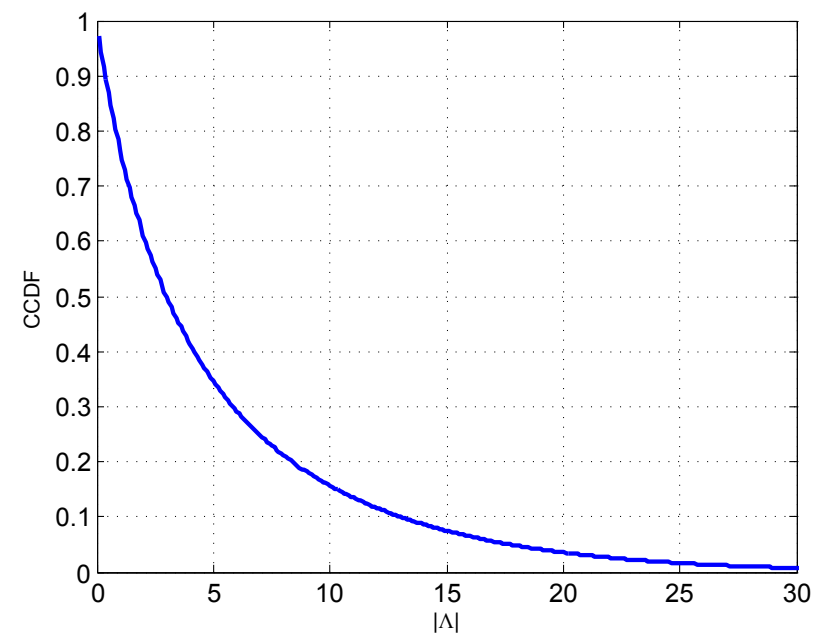

Figure 4.6: Cumulative distribution function of $|\Lambda|$ values for a received CNR of $11.25 \mathrm{~dB}$. 


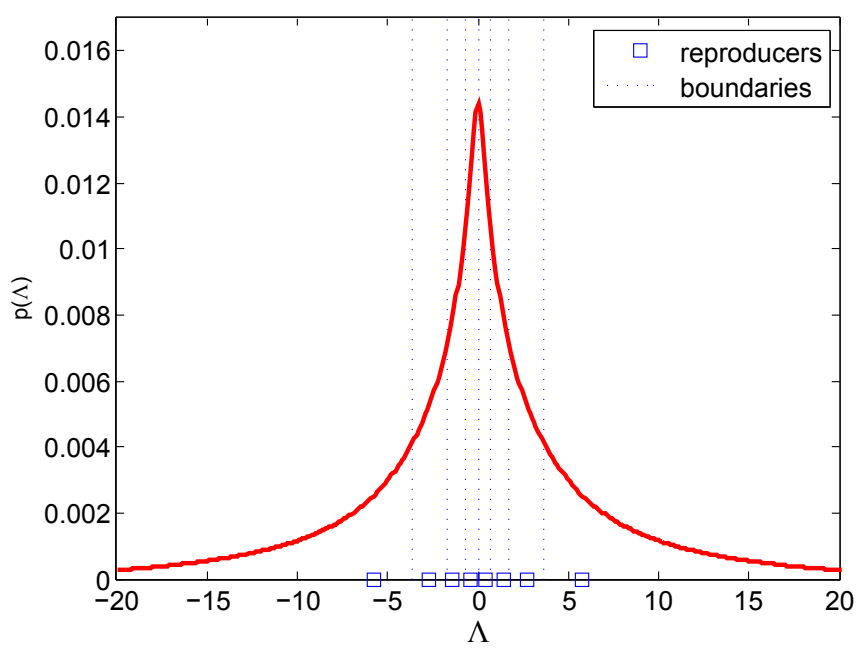

Figure 4.7: Example pdf of $\Lambda$ values for a received CNR of $11.25 \mathrm{~dB}$ with non-uniform quatization reproducers and boundaries designed with information bottleneck method.

value is selected such as with $10 \%$ probability all the received LLR values will have lower absolute value. This can be better observed with the Cumulative Density Function (cdf) of the absolute LLR values in Fig. 4.6. In this case, an absolute LLR value of approximately 13 fulfils this criteria. This $10 \%$ criteria is motivated by the fact that, in practical implementations, very high absolute LLR values are clipped prior to LDPC decoding.

\section{Non-Uniform quantization: Information Bottleneck Algorithm}

Next, the Information Bottleneck (IB) algorithm, used to maximize the data rate through the mutual-information $I(c ; d)$, is presented. [95] provides a detailed analysis of the IB method for quantization in the context of communication systems and specific implementation algorithms. Mutual-information based design for LLR quantization can outperform other classical approaches, such as MSE-optimal based quantizers where the goal is to represent the signal with low distortion $[95,110]$.

In [111], Tishby et al. introduced the notion of relevance through another variable. Relevant information in a random variable $\Lambda$ is defined as the mutual information this random variable provides about another random variable $c$, the relevance variable. For a fixed number of quantization levels, the aim of the IB method is to find a representation of $\Lambda$, which preserves as much information about $c$ as possible. Hence, the limited set of quantization levels 
constitutes a "bottleneck" through which we squeeze the information that $\Lambda$ provides about $c$. Since closed-form expressions for $p(c, \Lambda)$ are rarely available, $p(c, \Lambda)$ is approximated by measurement. $\Lambda$ is regarded as a discrete random variable in what follows.

The IB problem is given by

$$
p^{\star}(d \mid \Lambda)=\underset{p(d \mid \Lambda)}{\arg \min } I(\Lambda ; d)-\beta I(c ; d),
$$

where $\beta$ is the Lagrange multiplier of the relevant information $I(c ; d)$. In [111] it is shown that the optimal $p(d \mid \Lambda)$ is

$$
p(d \mid \Lambda) \propto p(d) \exp [-\beta \mathrm{D}(p(c \mid \Lambda) \| p(c \mid d))],
$$

where $\mathrm{D}(\cdot \| \cdot)$ denotes relative entropy. It must be emphasized that (4.6) is only a formal solution since $p(d)$ and $p(c \mid d)$ are defined implicitly in terms of $p(d \mid \Lambda)$. Then

$$
\begin{aligned}
p(d) & =\sum_{\Lambda} p(d \mid \Lambda) p(\Lambda), \\
p(c \mid d) & =\frac{1}{p(d)} \sum_{\Lambda} p(c, \Lambda) p(d \mid \Lambda) .
\end{aligned}
$$

The iterative IB algorithm consists of converging alternating iterations between (4.6)-(4.8), where $\beta$ allows to tradeoff quantization rate and relevant information. The IB algorithm converges to a locally optimal point. For $\beta \gg 1$ deterministic quantizer can be obtained. However, large values of $\beta$ can lead to numerical problems in the iterative IB algorithm. Therefore, this chapter uses the algorithm first proposed in [112] where a modified algorithm maximizes the relevant information for a given number of quantization levels and to take into account that the optimal quantizer is deterministic. A deterministic quantizer is returned upon convergence to a relative accuracy of $\varepsilon$ or otherwise after a predetermined number of iterations. The algorithm is executed with different, randomized initializations in order to obtain a good quantizer.

The relevant information $I(c ; d)$ does not depend on the values of $d$. Therefore the reproducer values need to be computed in a separate step after the quantizer design is completed:

$$
\lambda_{k}=\log \frac{p\left(c=1 \mid \Lambda \in \mathcal{I}_{k}\right)}{p\left(c=0 \mid \Lambda \in \mathcal{I}_{k}\right)}, \quad k=1,2, \ldots, n,
$$

where $\mathcal{I}_{k}, k=1,2, \ldots, n$ are the quantization regions defined by the designed boundaries. (4.9) ensures that the quantized LLRs are consistent. 
Fig. 4.7 shows the pdfs of the received LLR values with a CNR of $11.25 \mathrm{~dB}$ with 8 bpc for the MSB. Fig. 4.7 also includes non-uniform quantizer reproducers and boundaries designed with the IB algorithm described above. We can observe how the non-uniform quantizer gathers the reproducers closely around the absolute value zero, where the pdf has higher probability.

\subsection{Performance Evaluation of Quantized Ar- chitectures}

This section provides physical layer simulation results to illustrate the performance improvements by iterative detection and the performance degradation introduced by LLR and I\&Q quantization with different word lengths and algorithms. A summary of the simulation parameters is presented in Table 4.1. The simulated scenario is NGH vehicular MIMO $2 \times 2$ cross-polar channel model with $60 \mathrm{~km} / \mathrm{h}$ (cf. Appendix A). The simulations include inner LDPC codes with a word length size of 16200 bits with a code-rate $1 / 3$ and outer $\mathrm{BCH}$. The simulated system uses 4096 subcarriers and an 1/8 guard interval, to trade-off network cell area and resilience against Doppler spread in an $8 \mathrm{MHz}$ bandwidth. For the performance evaluation, TI blocks with 48 LDPC codewords are transmitted and decoded. These values have been selected as a trade-off between simulation time and performance accuracy. It was confirmed with simulations that using more LDPC codewords within the TI block did not have a significant impact in the final performance. To analyse the effect of the quantization with the constellation order three spectral efficiencies are evaluated, which are specified in DVB-NGH, i.e., 6 bpc (with QPSK and 16QAM constellations in the first and second transmit antennas, respectively), 8 bpc (with 16QAM constellation in both transmit antennas), and $10 \mathrm{bpc}$ (with 16QAM and 64QAM in the first and second transmit antennas, respectively). As specified in DVBNGH, Gray labelling is used and perfect CSI at the receiver side it is assumed. To provide reliable results, the performance under different channel realizations at each CNR is evaluated, i.e., different seeds for the (pseudo) random generators at each CNRs. On the other hand, for a given CNR all the receiver configurations were simulated under the same set of channel realizations, i.e., same seed for the (pseudo) random generator for a given CNR. Regarding the stopping criteria, for each CNR a maximum number of $20 \cdot 10^{3}$ and a minimum number of $2 \cdot 10^{3}$ TI blocks are simulated. The simulation is stopped when 200 erroneous TI Blocks have been detected. A TI Block is considered erroneous when a erroneous bit is detected in the entire TI block. Finally, the selected Quality of Service (QoS) for the comparisons is a BER of $10^{-5}$ before BCH decoding. This error criteria was selected as a compromise between simulation time and error criteria in mobile channels. 
4.3 Performance Evaluation of Quantized Architectures

Table 4.1: Summary of Simulation Parameters

\begin{tabular}{|c|c|}
\hline Simulation Parameters & Value \\
\hline Channel Model & NGH mobile with $60 \mathrm{kmph}$ \\
\hline Antenna configuration & MIMO $2 \times 2$ with spatial multiplexing \\
\hline MIMO precoding & 4096 subcarriers \\
\hline FFT size & $1 / 8$ \\
\hline Guard interval & $16200 \mathrm{bits}$ \\
\hline LDPC code length & $1 / 3$ \\
\hline Code-rate & $6 \mathrm{bpc}(\mathrm{QPSK}+16 \mathrm{QAM})$ \\
Spectral efficiencies & $8 \mathrm{bpc}(16 \mathrm{Q}$ AM+16QAM) \\
(and constellation in each transmit antenna) & $10 \mathrm{bpc}(16 \mathrm{QM}+64 \mathrm{QAM})$ \\
\hline Labelling & Gray labelling \\
\hline Channel estimation & perfect receive CSI \\
\hline QoS & BER $=10^{-5}$ before BCH \\
\hline
\end{tabular}

It is noted that since the LLR and I\&Q statistics depend on the received CNR, a different quantizer for each possible received CNR could be designed. However, in this section, only a single quantizer is designed for the CNR that reaches QoS performance with full resolution. This implies that at other received CNRs there will be a mismatch condition between the channel statistics and the statistics used for the design of the quantizers. Compared to a quantizer design that changes with the received CNR, the approach adopted in this chapter eases the hardware implementation at the expense of some performance degradation.

Figure 4.8 studies, for $8 \mathrm{bpc}$ and code-rate $5 / 15$, the performance gain due to iterative MIMO-BICM decoding with different number of outer iterations, i.e., number of soft information exchanges between the MIMO demapper and the LDPC decoder. In this analysis, the maximum complexity of the LDPC decoder is fixed to 50 iterations. The demapping and decoding of a given codeword is completed once the parity check conditions are satisfied. For example, for the curve labelled with " 5 outer by 10 inner" the demapper is executed a maximum number of 5 times and the LDPC decoder runs a maximum number of 10 iterations of the sum-product algorithm for each demapping execution. As we can observe, increasing the number of outer iterations enhances the BER performance. However, the performance improvement comes at the expense of higher computational complexity due to the repeated execution of the demapper block. With 5 outer iterations, the MIMO-BICM-ID gain is $0.8 \mathrm{~dB}$ and further increasing the number of outer iterations to 10 or 25 only provides a minor improvement. Since MIMO demodulation with high spectral efficiencies is one of the receiver components with highest complexity, in the rest of 


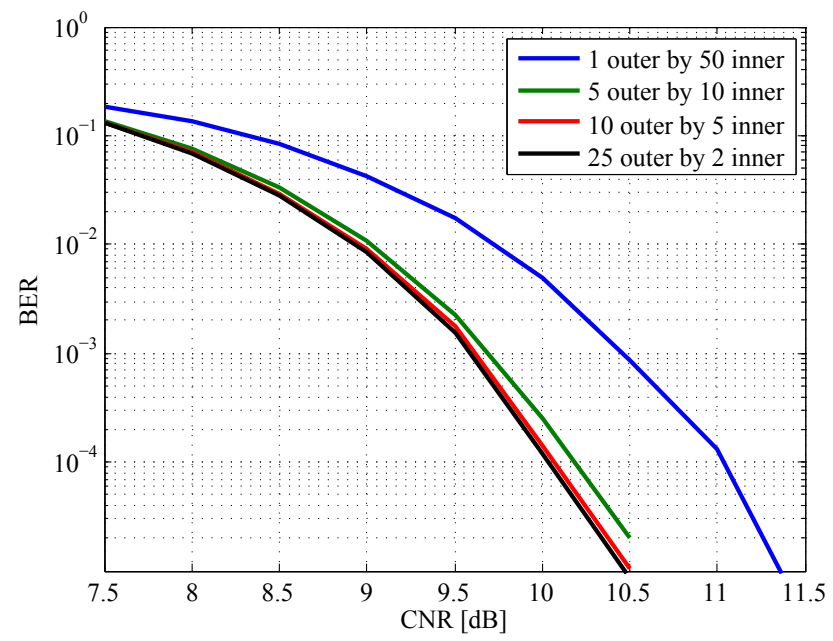

Figure 4.8: BER performance for MIMO-BICM with iterative decoding with different number of outer iterations. The maximum complexity of the number of LDPC decoding iteration is fixed to 50 .

evaluations the configuration 5 outer by 10 inner is selected, which provides a reasonable trade-off between receiver complexity and iterative decoding gain.

Figures 4.9, 4.10 and 4.11 present BER vs. CNR performance results with quantized LLRs for the three spectral efficiencies of 6,8 and $10 \mathrm{bpc}$, respectively. The performance of the LLR quantization algorithms presented in section 4.2.2 is included (uniform quantization with solid curves and non-uniform quantization with dashed lines). In particular, the degradation due to LLR quantization is studied with 2, 3 and 4 bits for each received LLR. As a baseline, the performance with full resolution is included with solid lines and circle markers. For the three spectral efficiencies the results show that the use of non-uniform quantization with IB algorithm provides a performance improvement compared to uniform quantization. In particular at coarse quantization levels (e.g., 2 bits per LLR) this gain is significant. In general for uniform quantization we can observe that the performance degradation compared to full resolution increases with increasing spectral efficiency. For instance, with 3 bits per LLR, the performance loss at QoS is $1 \mathrm{~dB}, 1.5 \mathrm{~dB}$ and more than $2 \mathrm{~dB}$ for 6,8 and $10 \mathrm{bpc}$, respectively. This increasing degradation can be explained due to the less dominant effect of the AWGN noise, which produces less uniform LLR distribution at higher spectral efficiencies. On the other hand, for nonuniform quantization, the loss remains approximately the same for the three spectral efficiencies. In particular, with 2 bits per LLR the loss is around $1 \mathrm{~dB}$, 


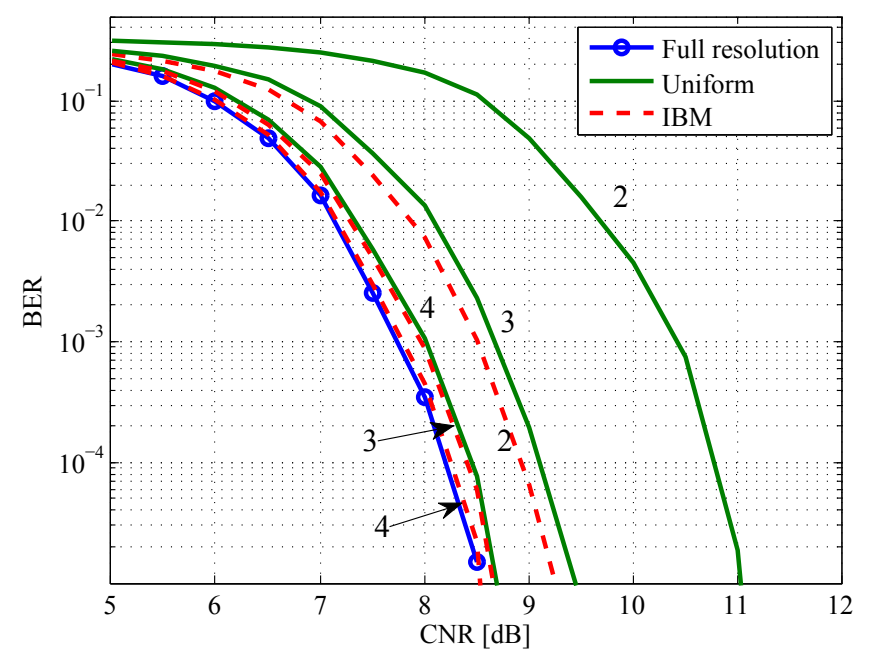

Figure 4.9: BER performance with quantized LLRs with 6 bpc. Uniform quantization and non-uniform quantization with IB method are included with 2, 3 and 4 bits per LLR.

for 3 bits per LLR the degradation is lower than $0.5 \mathrm{~dB}$ and with 4 bits per LLR the performance gets very close to the full resolution performance. The superior performance of non-uniform compared to uniform quantization is because non-uniform quantization exploits the signal statistics at each particular spectral efficiency.

Figures 4.12, 4.13 and 4.14 present BER vs. CNR performance results with quantized I\&Q components. In these evaluations iterative decoding is not implemented. The performance of uniform and non-uniform quantization is studied and further details of the I\&Q quantization algorithms are presented in section 4.2.1. For the three spectral efficiencies it can be observed that nonuniform quantization of I\&Q components reduces the degradation compared to uniform quantization, specially with coarse resolution. For instance, for 6 and $10 \mathrm{bpc}$ with 3 and 4 bits of quantization, respectively, non-uniform quantization outperforms uniform quantization by various $\mathrm{dBs}$. However, the performance difference between both approaches reduces at finer resolutions. For instance, at $8 \mathrm{bpc}$ with 4 bits of quantization a gain of $0.5 \mathrm{~dB}$ is achieved with nonuniform quantization. In general for the uniform quantizers the results show that the degradation increases with increasing spectral efficiencies, which can be seen in the degradation of 4 and 5 bits of quantization per I\&Q component. On the other hand, with non-uniform quantization, although the degradation 


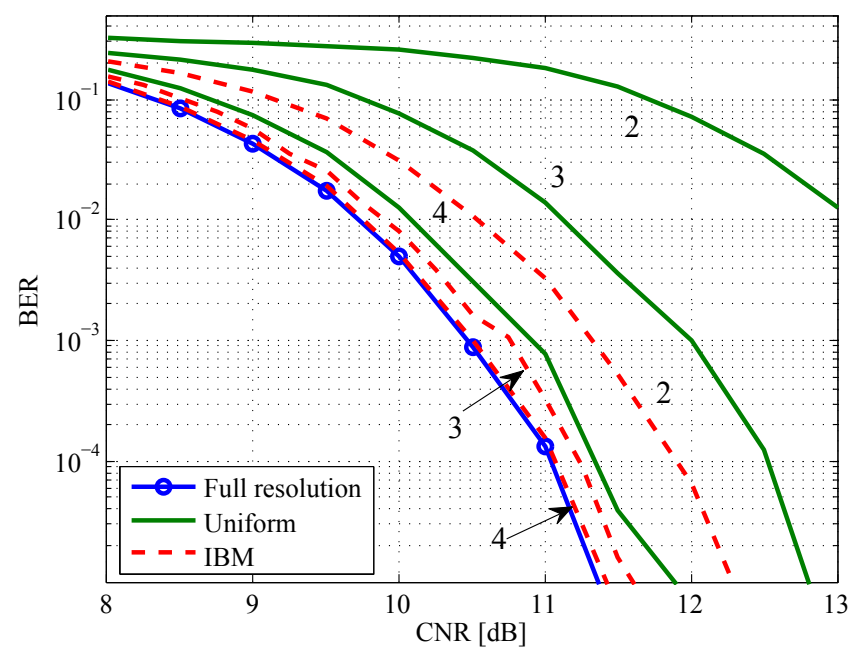

Figure 4.10: BER performance with quantized LLRs with 8 bpc. Uniform quantization and non-uniform quantization with IB method are included with 2, 3 and 4 bits per LLR.

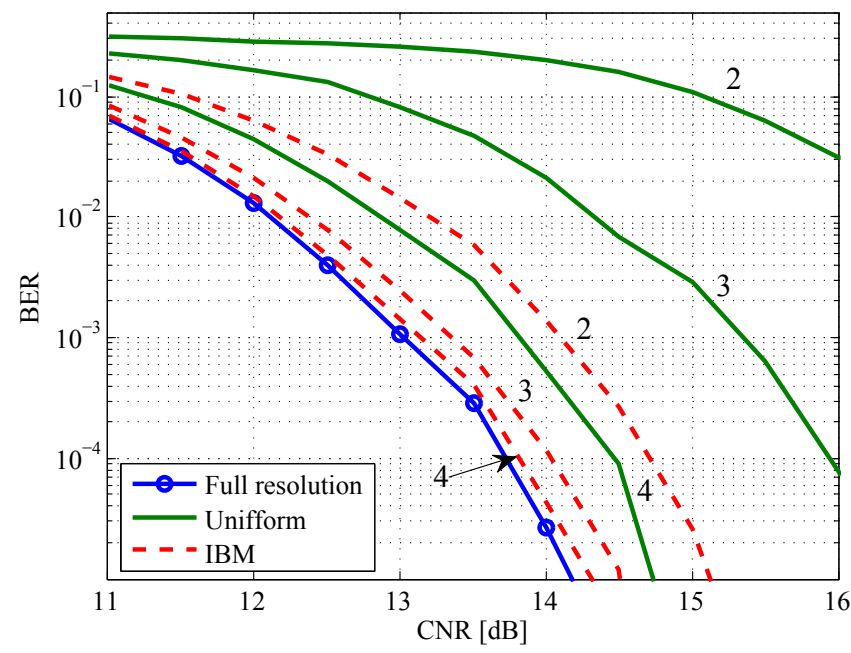

Figure 4.11: BER performance with quantized LLRs with 10 bpc. Uniform quantization and non-uniform quantization with IB method are included with 2, 3 and 4 bits per LLR. 


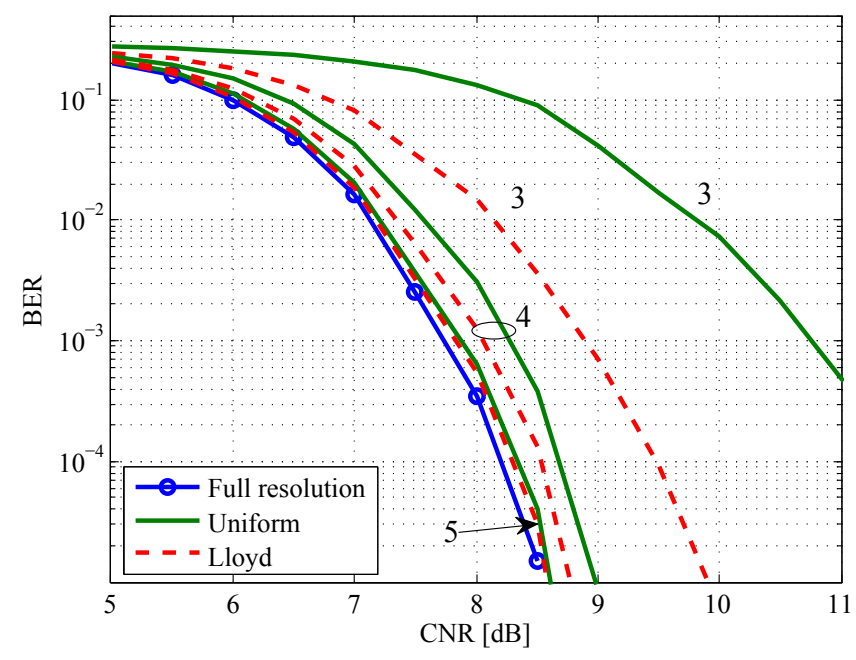

Figure 4.12: BER performance with quantized I\&Q with 6 bpc. Uniform quantization and non-uniform quantization with Lloyd-Max method are included with 3,4 and 5 bits per I or Q component.

also increases with the spectral efficiency, the loss is lower than the loss with uniform quantization.

Figures $4.15,4.16$ and 4.17 present BER vs. CNR performance results with quantized I\&Q components and iterative decoding. As baseline, the performance with full resolution for the iterative and non-interative set-up is included. This will allow us to study the MIMO-BICM-ID gain reduction due to quantization degradation. Overall, the performance degradation due to quantization with MIMO-BICM-ID is very similar to the degradation without iterative decoding. These results show that to maintain the MIMO-BICM-ID gain (with full resolution) of $0.7,0.76$, and $1.4 \mathrm{~dB}$ for 6,8 and $10 \mathrm{bpc}$, respectively, fine resolution is needed, e.g., 5 quantization bits per I\&Q component with nonuniform quantization.

\subsection{Memory vs. Performance Trade-Off}

DTT systems have to cope with signal strength fluctuations occurring during the motion of the receiver terminals, and this task is achieved with the TI block. The TI block interlaces multiple constellation symbols to increase the time diversity and hence, to increase the resilience against time selective fading. To provide sufficient time diversity DVB-NGH specifies a maximum interleaving 


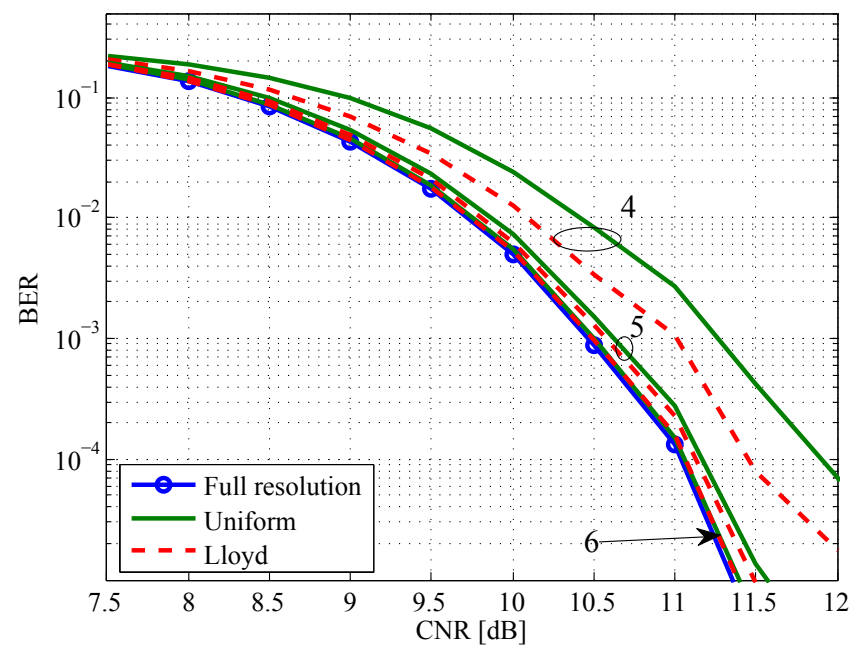

Figure 4.13: BER performance with quantized I\&Q with 8 bpc. Uniform quantization and non-uniform quantization with Lloyd-Max method are included with 4, 5 and 6 bits per I or Q component.

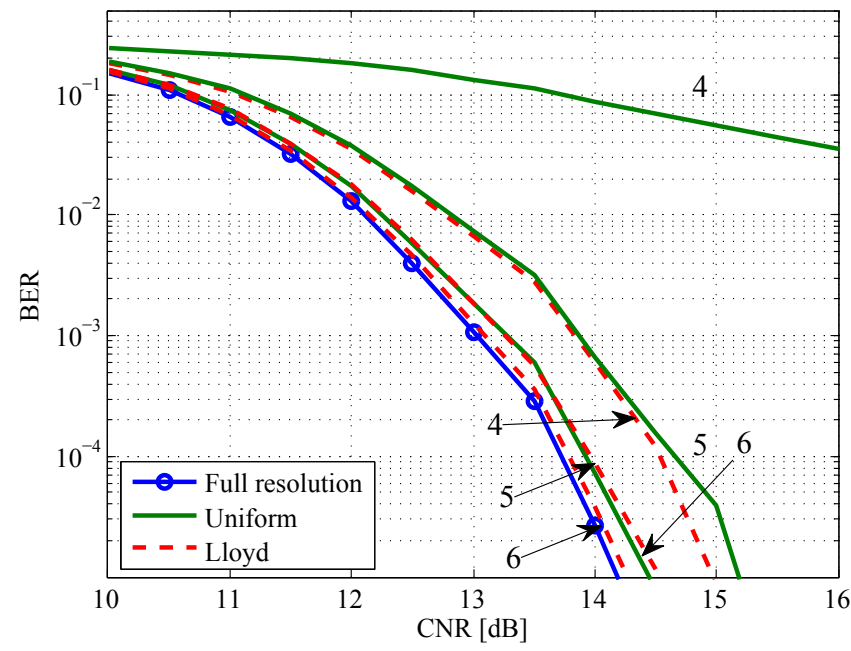

Figure 4.14: BER performance with quantized I\&Q with 10 bpc. Uniform quantization and non-uniform quantization with Lloyd-Max method are included with 4, 5 and 6 bits per I or Q component. 


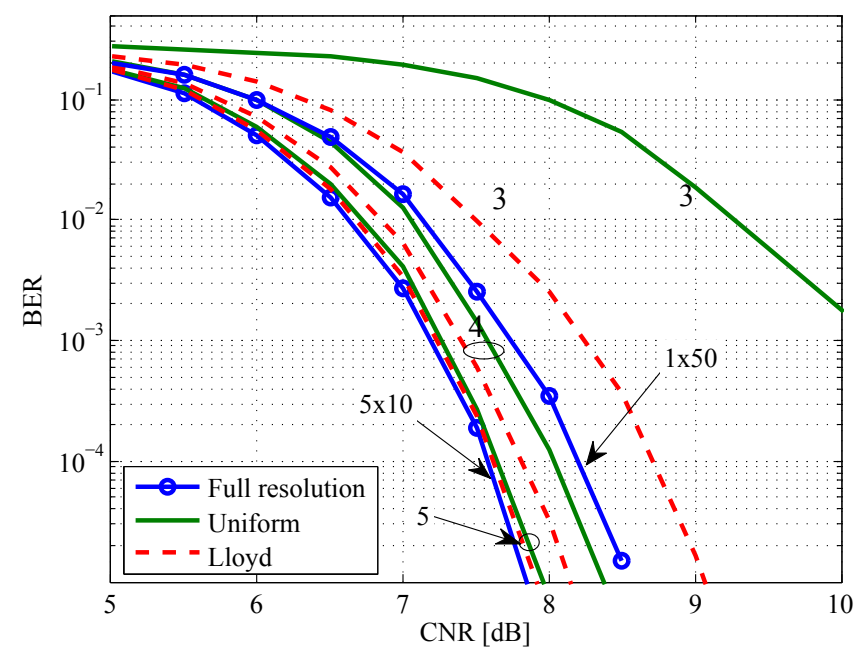

Figure 4.15: BER performance with quantized I\&Q with 6 bpc and iterative decoding. Uniform quantization and non-uniform quantization with Lloyd-Max method are included with 3, 4 and 5 bits per I or Q component.

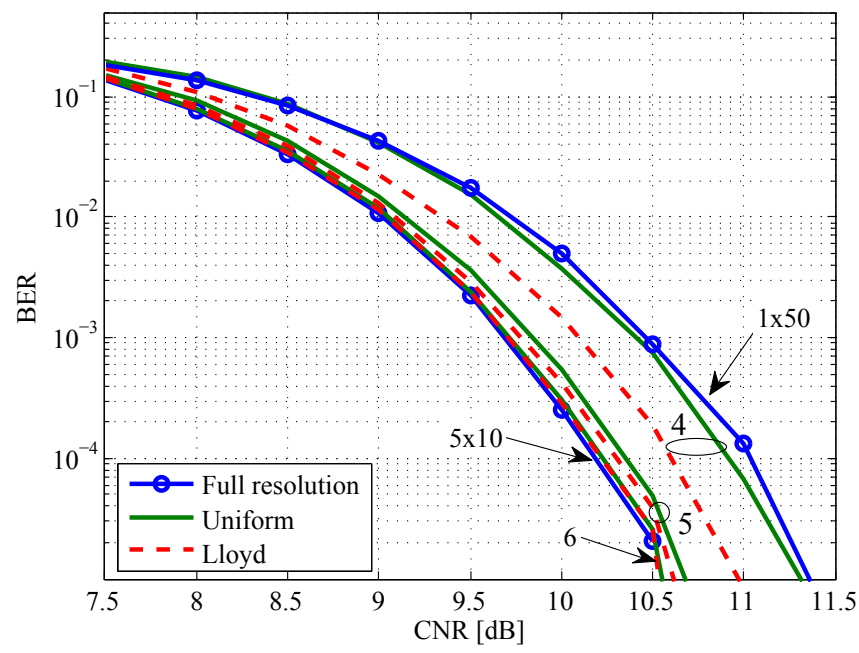

Figure 4.16: BER performance with quantized I\&Q with 8 bpc and iterative decoding. Uniform quantization and non-uniform quantization with Lloyd-Max method are included with 4, 5 and 6 bits per I or Q component. 


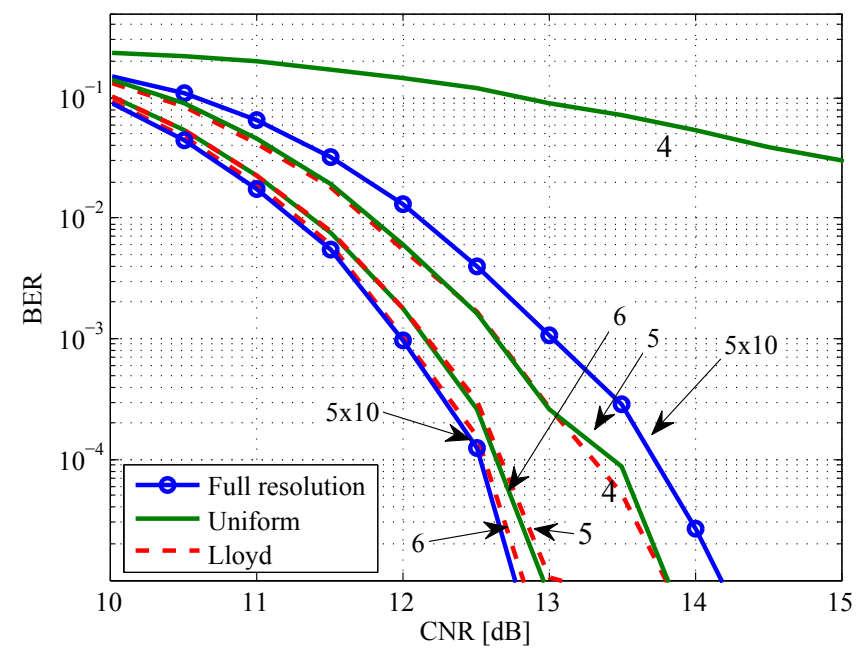

Figure 4.17: BER performance with quantized I\&Q with $10 \mathrm{bpc}$ and iterative decoding. Uniform quantization and non-uniform quantization with

Lloyd-Max method are included with 4, 5 and 6 bits per I or Q component.

depth of $2^{18}$ constellation symbols ${ }^{1}$. It is important to note that before the receiver can start decoding the received data, all the symbols corresponding to the TDI block memory need to be stored. The final TDI block memory size in bits will depend on the number of quantization levels applied to the received signal. In general the higher the number of quantization levels, the higher the fidelity, but on the other hand the higher the memory requirements. There is therefore a trade-off between in-chip memory and system performance.

Figure 4.18 illustrates the maximum number of transmit constellation symbols in a MIMO system with two transmit antenna streams. In DVB-NGH the maximum TI depth of the sheer terrestrial profile with single antenna transmissions is specified to $2^{18}$ constellation symbols. For MIMO with two transmit streams, since it requires (independent) constellation symbols at each transmit antenna, the MIMO TI depth is halved compared to the SISO profile. This is done as to maintain the same constrain in the maximum number of transmit constellation symbols within a TI block.

For each received channel realization, the receiver can store into the TDI memory different signal components, which would lead, as we will discuss later

\footnotetext{
${ }^{1}$ In DVB-NGH multiple sophisticated mechanisms of TI are defined such as the TI depth can be extended over multiple frames up to values around 2 seconds [47]. In the basic TI scheme, where contiguous constellation symbols from the same TI block are interlaced, $2^{18}$ cells correspond to approximately $40 \mathrm{~ms}$.
} 


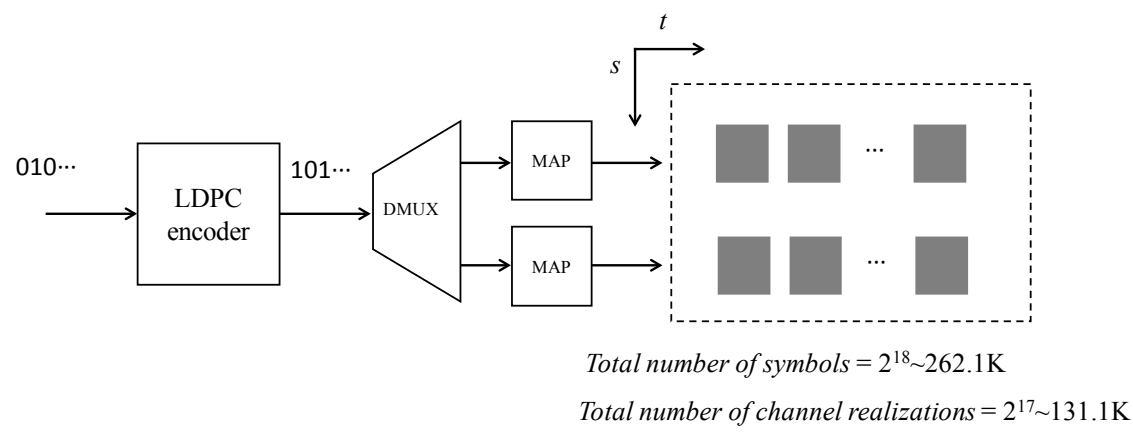

Figure 4.18: Transmit TI depth defined as the maximum number of constellation symbols.

in more detail, to different memory and performance trade-offs. In a MIMO $2 \times 2$ system, for each channel realization, the received signal components are: two distorted symbols (i.e., $y_{1}$ and $y_{2}$ ), one from each received antenna, and four channel estimates (i.e., $h_{11}, h_{12} h_{21}, h_{22}$ ). The first receiver architecture quantizes, for each signal component, the I and Q components. This is illustrated in the left part of Fig. 4.19. If $N_{B}$ bits are used to quantize each I and Q component, $M_{\mathrm{I} \& \mathrm{Q}}=6 \cdot 2 \cdot N_{B}$ bits of in-chip memory are required.

Alternatively, instead of quantizing I\&Q components, the received symbols $\mathbf{y}$ and channel estimates $\mathbf{H}$ are passed (with full resolution) to the demapper (which also operates with full resolution) and computes soft information in the form of LLRs. The output LLRs are the signal components to be quantized and stored in the in-chip TDI memory. For each received channel realization, the number of bits required to store the computed LLRs depend on: (i) the transmitted spectral efficiency (e.g., 6 LLRs for $6 \mathrm{bpc}$ ) and the number of bits employed to quantize each LLR. Hence, the memory $M_{\Lambda}$ per channel realization is: $M_{\Lambda}=N_{B} \cdot N_{\mathrm{bpc}}$, where $N_{\mathrm{bpc}}$ is the number of bpc. This is illustrated in the right part of figure 4.19.

Figures 4.20 to 4.22 study the trade-off between the required in-chip memory for TDI and system performance for the two quantization approaches and for the three spectral efficiencies of 6,8 and $10 \mathrm{bpc}$, respectively. Non-uniform quantization is used since it showed superior performance than uniform quantization in previous subsection. The performance with full resolution with iterative and non-iterative decoding is also included as a baseline. For the noniterative set-up, the performance with quantized I\&Q and LLR components is presented. On the other hand, for the iterative receiver, only the performance of quantized I\&Q is included since it is the only architecture that allows it. 

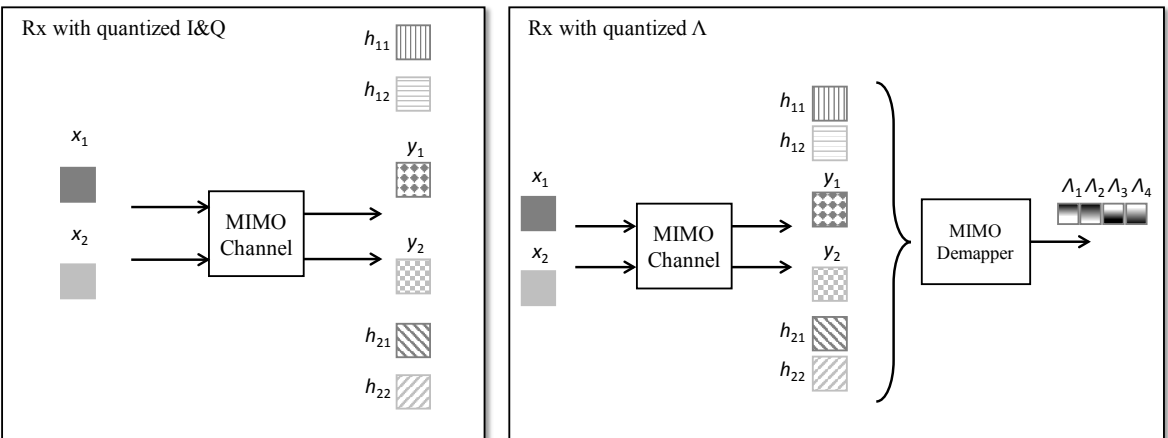

Figure 4.19: Diagram of quantized components for receiver architecture with quantized I\&Q components and a receiver architecture with quantized Log-likelihood rations.

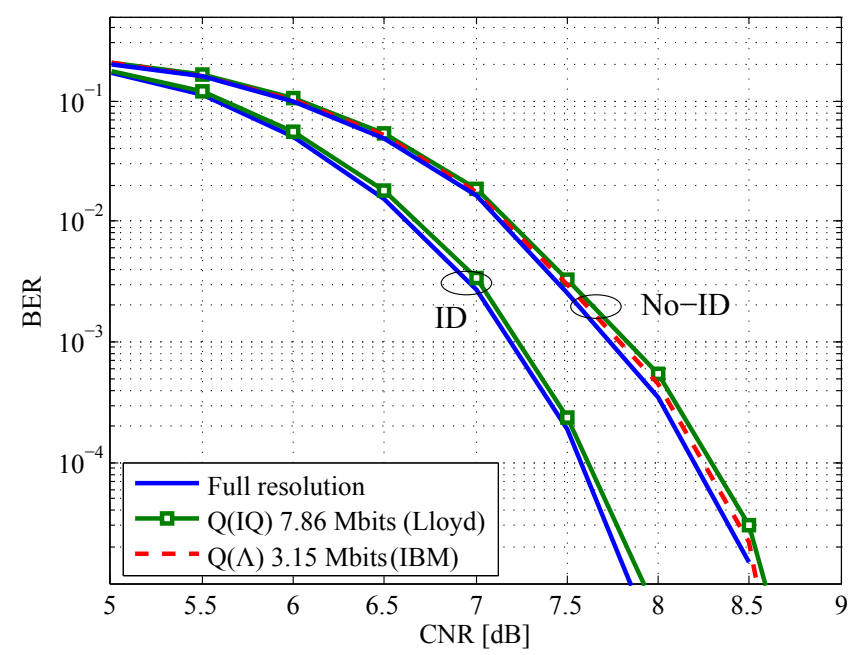

Figure 4.20: Performance comparison between LLR quantization and IQ quantization for $6 \mathrm{bpc}$. 


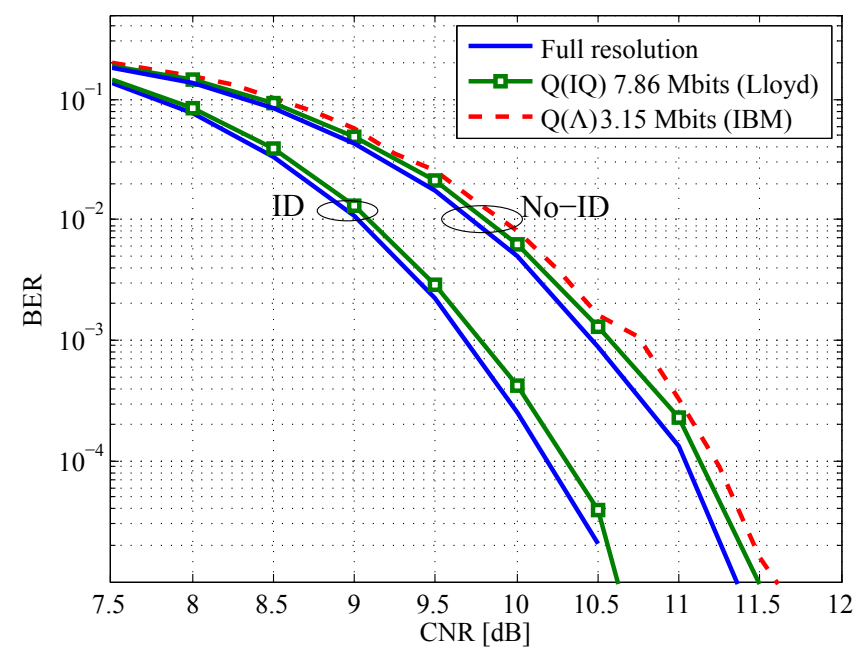

Figure 4.21: Performance comparison between LLR quantization and IQ quantization for $8 \mathrm{bpc}$.

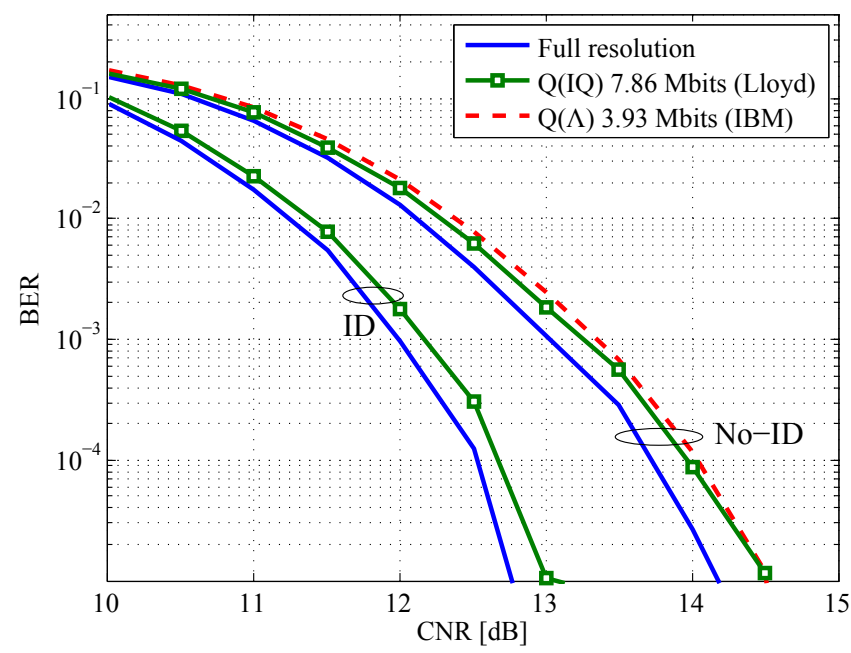

Figure 4.22: Performance comparison between LLR quantization and IQ quantization for $10 \mathrm{bpc}$. 
Table 4.2: Summary of performance degradations due to quantization in the considered receiver architectures and for different in-chip memory sizes.

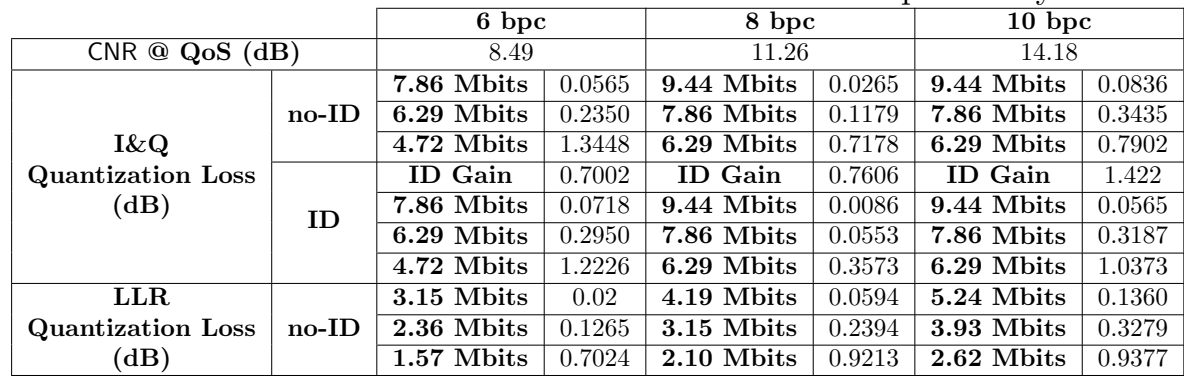

For the three spectral efficiencies, representative in-chip memory sizes with a performance degradation lower to $0.25 \mathrm{~dB}$ are included in the figures. (Additional in-chip memory sizes and associated degradation is presented in Table 4.2.) From the results it can be concluded that for similar quantization performance degradation, LLR quantization requires around half the memory compared to the in-chip memory with I\&Q quantization. More specifically, for 6 and 8 bpc, I\&Q quantization requires 2.5 times more in-chip memory and for 10 bpc, I\&Q quantization requires exactly double in-chip memory.

\subsection{Summary and Conclusions}

This chapter has assessed the design and performance evaluation of practical MIMO-BICM receivers based on quantized information. Two types of quantized receivers have been investigated: quantization of I\&Q samples and quantization of LLRs. The implications of each approach on the receiving architecture, the in-chip memory and the possibility of implementing iterative decoding have been presented and discussed. Using the DVB-NGH MIMO physical layer as reference, first, the performance gains of MIMO-BICM-ID have been evaluated. Additionally, the performance degradations of uniform quantization and non-uniform quantization algorithms have been studied showing significant potential benefits for non-uniform quantization adapted to the signal statistics. The results obtained in this chapter highlight the important trade-off between in-chip memory and performance for quantized MIMO-BICM receiver architectures.

The use of non-uniform quantizers adapted to the signal statistics provide significant improvements in terms of performance or alternatively in-chip memory savings. This has been verified for both quantized architectures, quantized I\&Q components and quantized LLRs, but showing higher enhancement for 
the latter. The higher memory requirements required by I\&Q quantization allow, on the other hand, to exploit the benefits of MIMO-BICM with iterative decoding. As shown in Table 4.2, the MIMO-BICM-ID gain is retained with quantization (and with sufficient resolution), which increases with increasing spectral efficiency $(0.7,0.8$ and $1.4 \mathrm{~dB}$ of gain for 6,8 , and $10 \mathrm{bpc}$, respectively). These additional gains not only come at the expense of additional in-chip memory, but also at the expense of additional demapping complexity.

In a receiver design constrained by in-chip memory, the best option is to quantized LLRs, since it entails the lowest memory requirements, although iterative decoding is not possible any more. In a receiver design constrained by demodulation complexity, the preferred option may also be quantized LLRs, since it would also provide additional in-chip memory savings. On the other hand, this chapter has only considered max-log demapper, which has high demodulation complexity. Other MIMO-BICM-ID demodulation algorithms are available in the literature, such as soft-in soft-out single-tree-search [41] or MMSE with priors (cf. Chapter 2 and $[89,44]$ ), which can significantly reduce the demapper complexity. The evaluation of the complexity of such demappers is out of the scope of this thesis. In a receiver design not constrained by either on in-chip memory or demodulation complexity, the preferred receiver architecture is to quantize I\&Q components since it also allows MIMO-BICMID, which can provide significant performance improvements. This type or receiver could be classified as an advanced receiver providing best performance with higher receiver complexity. 
CHAPTER 4. RECEIVER MEMORY AND PERFORMANCE TRADE-OFF OF SOFT-QUANTIZED INFORMATION 


\section{Chapter 5}

\section{Conclusions and Outlook}

T

HIS DISSERTATION has focused on transmit and receive signal processing for 1 digital terrestrial TV (DTT) systems with multiple-input multiple-output (MIMO) and bit-interleaved coded modulation (BICM) technologies, without a return channel from the receivers to the transmitters. MIMO offers multiple benefits such as additional received power thanks to the array gain, additional system resilience against signal fluctuations due to diversity gain and higher data rates through spatial multiplexing gain. These benefits can be achieved with the same transmit power and bandwidth requirements as in single antenna communications. However, these enhancements usually imply a higher system complexity at the transmitter and receiver sides. Due to the potential benefits, MIMO has been included for the first time as part of the DTT specifications DVB-NGH and ATSC 3.0 to overcome the Shannon limit of single antenna communications. These two specifications have adopted a MIMO configuration with two antennas at transmit and receiver ends (i.e., $2 \times 2 \mathrm{MIMO}$ ) able to transmit two independent data streams into the same time and frequency resources, i.e., spatial multiplexing gain of two. The operation in the UHF band of theses systems have motivated the adoption of cross-polar antenna configuration (i.e., antennas with vertical and horizontal polarizations). Crosspolar antennas can improve the performance under spatial correlated fading due to the orthogonal nature of the cross-polar channel, and also it can provide smaller handset device sizes compared to the co-polar configuration (i.e., antennas with the same polarization) which rely on antenna separation to provide independent fading signals.

The final MIMO gains directly depend on the intrinsic physical characteristics of the MIMO channel such as correlation among the spatial paths and transmit power imbalance between transmit antennas. To overcome the potential degradations, DVB-NGH and ATSC 3.0 have adopted precoding solutions 
based on rotation matrices which apply a linear transformation to the independent information streams to improve the performance. The three main components of these type of precoders are rotation matrix, stream-power-allocation, and power imbalance matrix (which can be considered as part of the precoder or as part of the channel). Additionally, since the broadcast network tends to exhibit common channel characteristics such as predominant line-of-sight (LOS) in rooftop environment, antenna correlation, or cross-polarization discrimination (XPD) factors, these characteristics could be used by a precoder to exploit such information statistics.

On the other hand, even in ideal channel conditions, MIMO-BICM receivers need sophisticated signal processing to extract the potential benefits of the MIMO channel. It is common practice during the standardization of new communication schemes to evaluate candidate transmission technologies with optimal receiving conditions such as optimal demodulation or infinite precision arithmetic. However, it is crucial for a good final system performance to carefully design specific receiving algorithms, which provide suitable performance while meeting complexity restrictions.

Signal quantization is a fundamental operation that can be found in any digital receiver, which permits transformation of continuous signals to a discrete signal sequence that can be processed, transmitted and/or stored in conventional digital processors. A fundamental trade-off connects quantizer resolution and distortion, hence, the quantization algorithms used at the receiver have a direct impact on the final performance and in-chip memory. Other important factor in MIMO-BICM receivers is that the performance can be significantly enhanced by iterative decoding algorithms, which exchange soft information between the demapper and the channel decoder in a iterative fashion. Apart from the additional computational complexity, this type of processing requires specific receiving architectures which can also have an impact on the in-chip memory.

At the transmitter side, the goal of this thesis has been to investigate and characterize the performance of MIMO precoding in DTT systems. First, this thesis has provided a performance characterization of the $2 \times 2 \mathrm{MIMO}$ precoding based on rotation matrices against: (i) channel parameters such as antenna orientation, spatial correlation, and transmit power imbalance, and (ii) transmission parameters such as constellation order and physical layer forward error correction (FEC) code-rate. Secondly, a MIMO-channel-precoding has been proposed that exploits statistical information of the MIMO channel. In this part of the thesis the DTT MIMO systems have been extended to support four independent information streams and its performance in DTT channels has been evaluated. The performance of the MIMO-channel-precoder is evaluated in a wide set of channel scenarios and mismatched channel conditions, (i.e., 
channel statistics and the statistics used for the design of the precoder differ), a typical situation in the broadcast set-up.

At the receiver side, the goal of this thesis has been to design and evaluate the performance of practical MIMO-BICM receivers based on quantized information. Two types of quantized receivers have been investigated: quantization of In-phase and Quadrature (I\&Q) samples and quantization of loglikelihood ratios (LLRs). The implications on the receiving architecture, the in-chip memory and the possibility of implementing iterative decoding have been investigated. Here, using the DVB-NGH MIMO physical layer as reference, first, the performance gains of MIMO-BICM with iterative decoding have been evaluated. Then, the performance of uniform and non-uniform quantization has been analysed showing potential benefits for non-uniform quantization adapted to the signal statistics. Finally, the in-chip memory size and system performance trade-off of quantized MIMO-BICM receivers has been presented and investigated.

This concluding chapter summarizes the main contributions of this dissertation and suggests further topics of research beyond the results of this work.

\subsection{Conclusions}

\section{MIMO Precoding for Terrestrial Broadcast Systems}

The research results obtained in this thesis, with the information-theoretic limits of BICM systems and BER simulations including channel coding, for $2 \times 2$ MIMO-BICM with Gray mapping and precoders based on rotation matrices show the following conclusions:

- In the case of co-polar antennas under i.i.d. fast fading Rayleigh channel, rotation does not have any effect in the final system performance. This channel model emulates a environment with co-polar antennas and rich scattering which already mixes the transmits information streams and a further linear transformation does not have a significant effect. Although this type of channel with co-polar antennas is not very representative for DTT systems, which relay in cross-polar antennas, it can provide insights for cross-polar channels with low XPD values (i.e., high coupling between polarizations). In the case that correlation between spatial paths exists, the results in this dissertation show that with high correlation condition, rotation can provide a significant improvements at all code-rates.

- In MIMO systems with cross-polar antennas under i.i.d. fast fading Rayleigh channel, rotation has an impact in the system performance. This type of channel is the most representative for DTT systems. As 


\section{CHAPTER 5. CONCLUSIONS AND OUTLOOK}

presented in Appendix A, mobile and fixed rooftop reception channels have high XPD values (i.e., low coupling between polarizations) with low spatial correlation.

In general, the optimal rotation angle depends on the FEC code-rate. In particular, the gain due to rotation increases as the code-rate increases, i.e., at low code-rates it is optimal not to rotate, and at mid and high code-rates it is optimal to rotate. The gain achieved with rotation, on the other hand, decreases with increasing constellation orders. The effect of power imbalance between transmit antennas shows that the rotation performance has the same behaviour as with equal power between transmit antennas. But in this case a higher rotation gain is achieved, which increases with the power imbalance between transmit antennas. This result suggests that the optimal rotation patterns obtained in this thesis can be resilient in the event of mismatch channel condition. The conclusions obtained for the optimal rotation tendency with both, mutual information analysis and numerical evaluations of system performance including bit-interleaving and FEC, presented a good alignment.

- This performance behaviour of the optimal rotation angles can be connected to other works in the literature. Previous works in $[63,104]$ have shown that while diversity techniques in MIMO systems with strong FEC codes improve performance at high code-rates, they can degrade the performance at low code-rates. And not only in MIMO systems, same behaviour in SISO systems with rotated constellations [80] has been reported. When the channel code has enough redundancy, additional diversity does not enhance the performance. On the contrary, when the system is configured with high code-rates (low redundancy levels), relaying on diversity techniques helps improving the performance. It is worth noting that the selection of constellation order and code-rate will depend on the requirements of the targeted services, i.e., improved robustness or high spectral efficiency.

- Additionally, results obtained during the standardization of the MIMO profile of ATSC 3.0 show same rotation behaviour as the one presented in this thesis. With 4 bits per channel use (bpc) (QPSK constellation at both transmit streams) and $8 \mathrm{bpc}$ (16QAM constellation at both transmit antennas), the optimized rotation angles in ATSC 3.0 under portable outdoor channels show the same tendency as the results reported in this thesis. Even with more sophisticated channel models and different transmit components, such as practical bit-interleaving implementations and non-uniform constellations, the conclusions still hold. 
- Stream-power-allocation connected to rotation has been investigated in the set-up of cross-polar antennas, i.i.d. fast fading Rayleigh channel and with transmit constellations in each antenna with different cardinality. In this scenario, the allocation of more power to the constellation with higher cardinality can improve the performance from mid to high code-rates. This can be achieved by transmit power imbalance or by stream-power allocation. The rotation in this case, allows for a flexible configuration of the desired power allocation to the information streams.

- The following remarks are given after a comparison of the precoding parameters adopted in DVB-NGH and ATSC 3.0 with the results obtained in this dissertation. The adopted parameters for the precoding in DVB-NGH can be suboptimal for some transmission configurations, since the same rotation angle has to be used for all system code-rates. For ATSC 3.0, since for each code-rate a different optimized rotation angle has been specified, it performs better providing optimal performance for each case. Although not included as basic part of the precoder in this dissertation, DVB-NGH and ATSC 3.0 include a phase-hopping (PH) term which applies a progressive change in the phase of the signal at the second transmit antenna. This $\mathrm{PH}$ has shown to be useful to improve the performance by avoiding any specific worst case channel condition over various channel realizations $[44,15]$. In the MIMO i.i.d. Rayleigh channels considered in this dissertation, it was confirmed with simulations that the introduction of the $\mathrm{PH}$ term did not have a significant effect in the final performance. Additionally, ATSC 3.0 included a new precoding component, the I/Q polarization interleaver, which is simply a switching interleaving operation between the real (In-phase) and imaginary (Quadrature) components of the two output symbols. Although included in the specification, no performance evaluation was done in connection to other precoder components.

- Furthermore, it is interesting to note that the rotation performance analysis with mutual information showed that the optimal rotation angles are different if the CM limit is computed. This means for a MIMOBICM receiver employing iterative decoding, the optimal rotation angle can change with the iterations as the performance approaches the CM bound.

The investigations regarding the proposed MIMO-channel-precoder that exploits statistical information of the MIMO channel for the terrestrial broadcasting setting show the following conclusions:

- This precoder has the potential to further increase the channel capacity when compared to the equivalent unprecoded MIMO set-up. The 
performance of the proposed channel-precoder is evaluated for fixed and portable channels and various reception conditions. A mismatched analysis allows the evaluation of the precoder performance when the channel statistics do not match the precoder statistics, a typical situation in the broadcast set-up. Capacity results present performance enhancements in scenarios with strong line-of-sight and correlated antenna component, and resilience against mismatched condition for the considered $4 \times 2 \mathrm{MIMO}$ systems. However, the MIMO-channel-precoder with $2 \times 2$ MIMO systems showed no performance enhancement.

- BER simulation results show, for the proposed MIMO-channel-precoder system, that for low code rates, enhancements can be achieved in the case of strong LOS correlation and resilience against mismatched condition with the channel statistics, a typical situation in the broadcast set-up.

\section{Receiver Memory and Performance Trade-Off of Soft-Quantized In- formation}

- The evaluations in this chapter have shown that the use of non-uniform quantizers adapted to the signal statistics provide significant improvements in terms of system performance or alternatively in-chip memory savings. This has been verified for both quantized architectures, quantized I\&Q components and quantized LLRs, but showing higher enhancement for the latter.

- An analysis of the receiving architectures with quantized I\&Q signal components and quantized LLRs unveils than the latter is not tailored to perform MIMO-BICM with iterative decoding. This is due to the exchange of demapping and time de-interleaving processing blocks. A receiving architecture with quantized I\&Q signal components does not have such a restriction since all the necessary signals (i.e., $\mathbf{y}$ and , $\mathbf{H}$ ) for demapping with prior information are quantized and stored into memory.

- Since the highest in-chip memory requirements are imposed by the time de-interleaving memory in DTT systems, this processing block is taken as reference for the memory size calculations. A further study of the in-chip memory requirements shows lower memory requirements for a receiving architecture with quantized LLRs than for a receiving architecture with quantized I\&Q signal components. Furthermore, the in-chip memory requirements with quantized LLRs depend only on the number of bits employed to quantize each LLR, and the number of bpc (e.g., $6 \mathrm{bpc}$ for QPSK+16QAM, $8 \mathrm{bpc}$ for 16QAM+16QAM, or $10 \mathrm{bpc}$ for $16 \mathrm{QAM}+64 \mathrm{QAM}$ for DVB-NGH). Since there is no dependence on the 
number of spatial paths, this architecture provides similar memory requirements to a SISO receiver. On the other hand, with quantized I\&Q signal components, the in-chip memory size does depend on the number of spatial paths. Due to the interrelationships between type of quantization architecture, MIMO-BICM with iterative decoding and in-chip memory, this thesis presents the in-chip memory vs. performance tradeoff to characterize MIMO-BICM receivers with quantized information.

- A receiver architecture with quantized I\&Q signal components has higher memory requirements than an receiver architecture with quantized LLRs. From the results it can be concluded that, for similar quantization performance degradation, LLR quantization requires around half the memory compared to the in-chip memory with I\&Q quantization. More specifically, for 6 and $8 \mathrm{bpc}$, I\&Q quantization requires 2.5 times more in-chip memory, and for $10 \mathrm{bpc}$, I\&Q quantization requires exactly double in-chip memory. On the other hand it is important to note that quantization of I\&Q signal components allows to exploit the benefits of MIMO-BICM with iterative demapping.

As shown in Table 4.2 (with sufficient resolution) the MIMO-BICM iterative decoding gain is maintained with quantization. This gain increases with increasing spectral efficiency: $0.7,0.8$ and $1.4 \mathrm{~dB}$ of gain for 6,8 , and $10 \mathrm{bpc}$, respectively, at BER $10^{-5}$. (These gains were obtained under a NGH mobile channel with $60 \mathrm{kmph}$.) However, these additional gains not only come at the expense of additional in-chip memory, but also at the expense of additional demapping complexity. Decreasing the quantization resolution can provide in-chip memory savings at the expense of a loss in the iterative decoding gain.

- If the receiver design is constrained by in-chip memory and/or demapping complexity, a receiver architecture with quantized LLRs is the preferred option. To reduce the demapping complexity, the receiver would not implement MIMO-BICM with iterative decoding, and in this case, LLR quantization provides with the lowest memory requirements.

- If the target is best receiving performance, a receiver architecture with quantized I\&Q signal components is the preferred option. Although this architecture has the highest in-chip memory requirements with the highest demodulation complexity, it also allows MIMO-BICM with iterative decoding which can provide significant performance improvements on top of the MIMO gain. 


\subsection{Outlook}

\section{Transmit Signal Processing for Terrestrial Broadcast Systems}

- In this dissertation, the investigated MIMO precoders have been optimized by maximizing the ergodic BICM mutual information for the precoders based on rotation matrices and by maximizing the ergodic capacity for the MIMO-channel-precoders. The ergodic criteria is the most appropriate measure for fast fading channels where within a code word multiple coherence periods of the channel occur. In static and portable channels, where the channel remains constant for the entire code word, the outage probability and the outage capacity are better measures, which are also commonly used in network planning [113].

The design of MIMO precoders with outage probability and outage capacity as design criteria is an interesting research topic. As studied in this dissertation, the optimization can take into account, amongst others, important channel parameters such as: transmit power imbalance, transmit asymmetric constellations, constellation cardinalities and FEC code-rate. Although, the results presented in this dissertation have shown performance improvements by precoding in static and portable channels, additional enhancements with system performance closer to the theoretical limits could be achieved with a precoder design based on outage criteria.

- The optimal rotation angles based on mutual information for a CM system have been shown to differ to the optimal angles for a BICM system. This implies that the rotation performance for a MIMO-BICM receiver implementing iterative decoding could change with the iterations. It thus seems very interesting to investigate the impact of the iterative MIMO decoding process in the MIMO rotation performance.

- The constellations used in this thesis are multilevel QAM with an uniform distance between constellation points. However, uniform constellations are suboptimal from the information theoretic point of view since to reach the channel capacity limit a Gaussian like distributed transmitted signal is required. Non-uniform constellations are designed to overcome this limitation and have been recently adopted for ATSC 3.0. MIMO precoding based on rotation matrices combined with non-uniform constellations has been adopted in ATSC 3.0 and reported in [15]. However, the non-uniform constellations adopted for the MIMO profile in ATSC 3.0 are reused from the SISO profile for which the non-uniform constellation have been optimized. Results in [15] show very similar rotation performance to the results presented in this thesis for QAM constellations. 
However, it would be interesting to study the relationship between the MIMO precoder based on rotation matrices and non-uniform constellations designed for the MIMO channel. Alternatively, a joint design of non-uniform constellations and precoding in terrestrial broadcast systems is an open research topic.

- Layer division multiplex (LDM) is a physical layer transmission technique to multiplex multiple services with different robustness and throughputs in the same time and frequency resource $[114,115]$ outperforming orthogonal time and frequency division multiplex. LDM has been included as a SISO baseline technology in ATSC 3.0 with two layers, one targeting mobile services (upper layer) and one targeting high data rate services (lower layer). The upper layer is configured with a robust modulation and code-rate to be able to operate in mobile environments and in the presence of interference. The lower layer can be configured with higher order modulation and code-rate to deliver higher throughput. A mobile receiver can decode its intended signal treating the lower layer as interference. A receiver targeting the lower layer, first needs to decode the upper layer, then use the decoded upper layer to remove it from the main received signal and then decode the lower layer signal. A natural extension of LDM and a important research topic is to include MIMO technology. Some initial investigations are reported in [116]. MIMO can be used in a LDM system to improve the resilience of the upper layer and/or to increase the data rate of the lower layer. There are system complexity implications such as the inclusion of additional transmit and receive antennas and additional demapping complexity which needs to be considered for a MIMO LDM system. Additionally, transmit signal design targeting layers with different optimization criteria, e.g., ergodic measures for mobile reception and outage probability for portable and static reception, is and interesting research problem.

\section{Receive Signal Processing for Terrestrial Broadcast Systems}

- This work has focused on quantization of I\&Q signal components and LLRs for MIMO systems with uniform constellations and due to the recent adoption of non-uniform constellations in broadcasting standards is interesting to extend this work to non-uniform constellations. Nonuniform constellations are designed per code-rate basis due to the different CNR thresholds which lead to different constellation diagrams. Hence, a different quantizer needs to be designed for each of the possible defined constellations. Additionally, constellations designed with robust coderates tend to condensate some of the constellation points which leads to a predominant contribution of the MSBs and marginal contribution of 
the LSBs to the average mutual information [80, 117]. This property can be used to reduce the MIMO demapping complexity by avoiding the calculation of distances for condensed constellation points (as a MIMO extension to the work reported in [80]). Additionally, this property can also be used to be exploited by the quantizer design by reducing the number of quantization levels for the LSBs and hence reduce the final in-chip memory.

- The evaluation of the gains achieved by MIMO-BICM with iterative decoding with non-uniform constellations is a straightforward evaluation, which can provide interesting results. An interesting analysis is the investigation on how the shaping gain behaves in a MIMO-BICM receiver with iterative decoding due to the different significance of the bits at the label with non-uniform constellations, which in turn also depend on the selected code-rate.

- From a system level perspective it would be desirable to have demodulators which directly produce quantized LLRs, instead of computing them at full resolution before quantization. For a specific variant of the sphere decoder [108, 118] and for a linear MMSE receiver [109] there exist such demodulators. However, the development of soft-output demodulators with quantized output is an interesting research topic.

- In Chapter 4, it has only been considered the max-log demapper which has demodulation complexity which increases exponentially with the number of transmit antennas. Other soft-in soft-out demodulation algorithms are available in the literature such as soft-in soft-out single-tree-search [41] or MMSE with priors (cf. Chapter 2 and $[89,44]$ ) which can significantly reduce the demapper complexity. A performance vs. complexity trade-off of soft-in soft-out demappers is provided in [119] and an extension including the in-chip memory due to soft-quantized information is an interesting research work.

- The demapper used in Chaper 4 is the soft-in soft-out max-log demodulator (cf. Section 2.4.4), which assumes that the input $\mathbf{y}$ is Gaussian distributed (2.42). However, in a practical receiver with quantized I\&Q signal components, the input of the demodulator is $\mathbf{y}_{q}$, a quantized version of $\mathbf{y}$. Since $\mathbf{y}_{q}$ is no longer Gaussian distributed, this produces a mismatched demapper. A further improvement to the results in Chapter 4 requires the computation/estimation of $p\left(\mathbf{y}_{q} \mid \mathbf{x}, \mathbf{H}\right)$ so the demapper takes into account the distribution of the quantized variable $\mathbf{y}_{q}$. Although the performance difference between the mismatched demapper and the modified demapper could reduce with the number of quantization levels, 
using this modified demapper can provide performance improvements at coarse quantization levels.

- The non-uniform quantizer design for I\&Q signal components in Chapter 4 has been based on the Lloyd-Max algorithm which minimizes the meansquared-error (MSE). An interesting variant to this solution is to apply the same approach as for the quantization of LLRs by maximizing the end-to-end rate through the mutual information of the quantized I\&Q channel outputs. The method in [120] or an a solution based on the information bottleneck method are promising ways to extend this work. 
CHAPTER 5. CONCLUSIONS AND OUTLOOK 


\section{Appendix A}

\section{MIMO Channels in Terrestrial Broadcasting: Models and Capacity}

7 HIS APPENDIX presents the potential gains of multi-antenna techniques in 1 terms of ergodic and outage capacities for representative channel models in terrestrial broadcast TV systems. First, it provides an evaluation with some basic channels widely used within literature and then proceeds with the analysis with channel models extracted from channel measurement campaigns in the UHF band. The focus is given on the potential gains over the single antenna transmitter and receivers, which is the most representative case in the current DTT deployment worldwide. The performance evaluation considers the case where the information of the channel state is available only at the receiver (CRSI) and when it is also available at the transmitter (CTSI). 


\section{A.1 Channel Models}

\section{A.1.1 Basic Models}

Correlated Co-polar MIMO Channel Using the model in (2.7) a general model for the complex baseband received signal is $\mathbf{y}=\mathbf{H s}+\mathbf{w}$, where $\mathbf{H}$ is the channel matrix with $N_{t}$ transmit by $N_{r}$ receive antennas, $\mathbf{s}$ is the $N_{t} \times 1$ transmitted vector, and $\mathbf{w} \sim \mathcal{C N}\left(\mathbf{0}, \sigma^{2} \mathbf{I}\right)$ is $N_{r} \times 1$ additive circularly symmetric complex Gaussian noise, where $\sigma^{2}$ is the noise power. As described in Chapter 2, when the MIMO system has antennas sufficiently separated, equally polarized and with many scatters the elements of the matrix $\mathbf{H}$ can be modelled with i.i.d. zero-mean complex Gaussian components, i.e., $h_{i j} \sim \mathcal{C N}(0,1 / 2), i=1 \ldots N_{r}, j=1 \ldots N_{r}$. Fading components with such characteristics are commonly known as Rayleigh distributed and it will denoted with the following matrix notation: $\mathbf{H}_{w}$.

In the case of co-polarized antennas, reaching independent fading depends on the coherence distance of the channel. This is defined as the maximum spatial separation over which the channel can be assumed constant, and is characterized by the angular spread, which in turn depends on the local scattering environment and the carrier frequency. In order to ensure independent fading across antennas, it is necessary that the separation between antennas is larger than the coherence distance. For a mobile which is near the ground with many scatterers around, the channel decorrelates over shorter space distances, and typical antenna separations of half to one carrier wavelength are sufficient. For transmit towers, larger antenna separations of several to tens of wavelengths may be required as a result of the longer distance between the scatterers and the antennas [26].

In the situation that the transmit and receive antennas are not sufficiently separated, and receiver and/or many scattered components come from the same direction, the channel components $h_{i j}$ are correlated. The correlation effect can be included by the following operation to the matrix $\mathbf{H}_{w}$ :

$$
\operatorname{vec}(\tilde{\mathbf{H}})=\mathbf{R}^{1 / 2} \operatorname{vec}\left(\mathbf{H}_{w}\right)
$$

where $\mathbf{R}$ is the $N_{t} N_{r} \times N_{t} N_{r}$ covariance matrix which describes the correlation between the channel paths. The operator $\operatorname{vec}(\cdot)$ is the linear transformation which converts the $N_{r} \times N_{t}$ matrix into a $N_{r} N_{t} \times 1$ column vector obtained by placing the columns of the matrix on top of one another. The term $\mathbf{R}^{1 / 2}$ is the Cholesky decomposition of the covariance matrix. In the case of uncorrelated channel components the matrix $\mathbf{R}=\mathbf{I}$.

Correlated Cross-polar MIMO channel The matrix $\tilde{\mathbf{H}}$ models the case of correlated fading with co-polar antennas either horizontal or vertical. An 
alternative configuration is to use antennas with different polarization, i.e., one transmit/receive antenna with vertical polarization and the other transmit/receive antenna with horizontal polarization and vice-versa. A change to cross-polar configuration can be made through a matrix $\mathbf{X}$ :

$$
\mathbf{X}=\left[\begin{array}{ccc}
x_{11} & \cdots & x_{1 N_{t}} \\
\vdots & \ddots & \vdots \\
x_{N_{r} 1} & \cdots & x_{N_{r} N_{t}}
\end{array}\right]
$$

where $x_{i j}=\left|h_{i j}\right| /\left|h_{\text {ref }}\right|$. Here $\left|h_{\text {ref }}\right|$ is the amplitude of the reference channel that in this thesis is chosen as $h_{\text {ref }}=h_{11}$. In the particular case of a $2 \times 2$ cross-polarized MIMO system the matrix $\mathbf{X}$ is [121]:

$$
\mathbf{X}=\left[\begin{array}{ll}
\left|h_{11}\right| /\left|h_{11}\right| & \left|h_{12}\right| /\left|h_{11}\right| \\
\left|h_{21}\right| /\left|h_{11}\right| & \left|h_{22}\right| /\left|h_{11}\right|
\end{array}\right] .
$$

Next the Cross Polarization Discrimination (XPD) factor is defined as the average power ration of the co-polar channel component and the cross-polarized channel component. In particular for the $2 \times 2$ cross-polarized MIMO case $\mathrm{XPD}=1 / X=\left|h_{11}\right|^{2} /\left|h_{21}\right|^{2}=\left|h_{11}\right|^{2} /\left|h_{12}\right|^{2}$. High/low XPD values represent low/high coupling between polarizations, e.g., $\mathrm{XPD}=1$ full coupling (pure co-polar) and XPD $=\infty$ no coupling (two parallel channels without interference). The cross-polar matrix $\tilde{\mathbf{H}}_{\times}$with correlated antennas can be expressed as $\tilde{\mathbf{H}}_{\times}=\mathbf{X} \odot \tilde{\mathbf{H}}$, where operator $\odot$ represents the Hadamard of element-wise multiplication.

Low fading correlation with cross-polarized antennas relays on the fact that the fading experimented by each polarization path possesses a low degree of correlation.

Line-of-Sight component The previous models represent the scenario where there is NLoS between the transmitter and the receiver. In the case that this LoS component exists, the previous models can be extended:

$$
\overline{\mathbf{H}}=\left[\begin{array}{ccc}
\exp \left(i \theta_{11}\right) & \ldots & \exp \left(i \theta_{1 N_{t}}\right) \\
\vdots & \ddots & \vdots \\
\exp \left(i \theta_{N_{r} 1}\right) & \ldots & \exp \left(i \theta_{N_{r} N_{t}}\right)
\end{array}\right]
$$

where $\theta_{i j}$ is a fixed random phase uniformly distributed between $[0,2 \pi)$. Similarly to the NLoS component, one can obtain a LoS component for cross-polar antennas through the matrix $\mathbf{X}$ as $\overline{\mathbf{H}}_{\times}=\mathbf{X} \odot \overline{\mathbf{H}}$. Finally, the channel matrix $\mathbf{H}$ is i.i.d. Rice distributed and can be further expressed as [121]:

$$
\mathbf{H}=\sqrt{\frac{K}{1+K}} \overline{\mathbf{H}}_{\times}+\sqrt{\frac{1}{1+K}} \tilde{\mathbf{H}}_{\times},
$$




\section{APPENDIX A. MIMO CHANNELS IN TERRESTRIAL BROADCASTING: MODELS AND CAPACITY}

where $K$ factor describes the power ratio between the LoS and NLoS components.

\section{A.1.2 Modified Guilford Rooftop Model}

This channel characterizes a rooftop reception environment, based on the model in [22] and extracted from a channel sounding campaign in Guildford, UK [21] of a MIMO $2 \times 2$ channel with cross-polar antennas arrangement. The MGM (Modified Guilford Channel) in [122] is made up of 8 taps with different values of delay and power gain. While the first tap is Rice distributed with $K$ factor, the rest are Rayleigh distributed. Each tap has a specific $X$ factor (cross-polar power ratio) describing the energy coupling between cross-polarized paths. The transmit antennas are co-located in a single transmitter site which cause at the receiver locations impinging signals with same strengths, arriving at the same time, and with no frequency offsets due to a common transmit local oscillator [45].

Power delay profile Specific values of the power delay profile and $X$ factors extracted from [123] and are presented in Table A.1.

Table A.1: Power delay profile of the MGM channel model, extracted from [123]

\begin{tabular}{|c|c|c|c|c|}
\hline Tap numper, $p$ & Excess delay, $\tau_{p}(\mu s)$ & $\begin{array}{c}\text { Co-polar power gain } \\
\sigma_{11}^{2}\left(\tau_{p}\right)=\sigma_{22}^{2}\left(\tau_{p}\right) \mathrm{dB}\end{array}$ & $\begin{array}{c}\text { Cross-polar power gain } \\
\sigma_{12}^{2}\left(\tau_{p}\right)=\sigma_{21}^{2}\left(\tau_{p}\right) \mathrm{dB}\end{array}$ & $X_{j}$ \\
\hline $1 \mathrm{LoS}$ & 0 & -0.943 & -16.13 & 0.0303 \\
\hline $1 \mathrm{NLoS}$ & 0 & -8.110 & -20.54 & 0.0572 \\
\hline $2 \mathrm{NLoS}$ & 0.2632 & -22.97 & -32.24 & 0.118 \\
\hline $3 \mathrm{NLoS}$ & 0.5264 & -32.27 & -39.1 & 0.208 \\
\hline $4 \mathrm{NLoS}$ & 0.7896 & -35.59 & -41.48 & 0.257 \\
\hline $5 \mathrm{NLOS}$ & 1.448 & -37.0 & -42.64 & 0.272 \\
\hline $6 \mathrm{NLoS}$ & 2.501 & -38.5 & -44.09 & 0.277 \\
\hline $7 \mathrm{NLoS}$ & 3.554 & -40.88 & -46.27 & 0.288 \\
\hline $8 \mathrm{NLoS}$ & 4.607 & -43.94 & -49.0 & 0.313 \\
\hline
\end{tabular}

Spatial correlation The model also exhibits spatial correlation between the antennas represented with a covariance matrix per tap:

$$
\mathbf{R}_{p}=\left[\begin{array}{cccc}
1 & 0.125 \sqrt{X_{p}} & 0 & 0.15 \\
0.125 \sqrt{X_{p}} & X_{p} & 0 & 0 \\
0 & 0 & X_{p} & 0.125 \sqrt{X_{p}} \\
0.15 & 0 & 0.125 \sqrt{X_{p}} & 1
\end{array}\right]
$$


The MGM is characterized by a prominent LoS component with low $X$ values, i.e., low coupling between vertical an horizontal components. The overall values for the $K$ and $X$ factors are 5 and 0.03 , respectively.

Signal model The time domain channel model for $2 \times 2$ MIMO is expressed in matrix form as:

$$
\mathbf{H}(t, \tau)=\left[\begin{array}{ll}
h_{11}(t, \tau) & h_{12}(t, \tau) \\
h_{21}(t, \tau) & h_{22}(t, \tau)
\end{array}\right]
$$

where $h_{i j}(t, \tau)$ is the impulse response between the $j^{\text {th }}$ transmit antenna and the $i^{\text {th }}$ receive antenna, $t$ is the time index, and $\tau$ a delay time. Furthermore, the channel can be decomposed into a LoS and NLoS components in vector form as follows [123], [22]:

$$
\operatorname{vec}\left(\mathbf{H}^{T}(t, \tau)\right)=\overline{\mathbf{h}} \delta(t)+\sum_{p=1}^{8} \mathbf{R}_{p}^{1 / 2} \mathbf{h}_{w, p} \delta\left(t-\tau_{p}\right)
$$

where $\overline{\mathbf{h}}$ is the vectorized LoS term, and the summation over the eight taps corresponds to the vectorized NLoS term. For the $p^{\text {th }}$ tap, the $N_{t} N_{r} \times N_{t} N_{r}$ covariance matrix $\mathbf{R}_{p}$ describes the correlation between the channel paths, $\mathbf{h}_{w, p}$ is i.i.d complex Gaussian random vector of size $N_{t} \times 1$, and $\tau_{p}$ is its time position defined in Table A.1.

Log-normal fading Additionally, the MGM model includes a $5.5 \mathrm{~dB}$ standard deviation log-normal distributed fading contribution into the LoS component to represent various network locations providing into a single model local and regional variations of the fading. The term $\overline{\mathbf{h}}$ is:

$$
\overline{\mathbf{h}}=\frac{1}{\sqrt{2.23}}\left[\begin{array}{c}
\lambda_{1} \sigma_{11}\left(\tau_{1}\right) \exp \left(i \theta_{11}\right) \\
\lambda_{2} \sigma_{12}\left(\tau_{1}\right) \exp \left(i \theta_{12}\right) \\
\lambda_{3} \sigma_{21}\left(\tau_{1}\right) \exp \left(i \theta_{21}\right) \\
\lambda_{4} \sigma_{22}\left(\tau_{1}\right) \exp \left(i \theta_{22}\right)
\end{array}\right]
$$

where

- $\lambda_{k}=10^{u_{k} / 20}, k=1, \ldots, 4$,

- $\mathbf{u}=\mathbf{W}^{1 / 2} \mathbf{q}$

- $\mathbf{q}=\left[q_{1}, q_{2}, q_{3}, q_{4}\right]^{T}$ is i.i.d. Gaussian elements with standard deviation of $5.5 \mathrm{~dB}$, and 


$$
\mathbf{W}=\left[\begin{array}{llll}
1 & \alpha & \alpha & \alpha \\
\alpha & 1 & \alpha & \alpha \\
\alpha & \alpha & 1 & \alpha \\
\alpha & \alpha & \alpha & 1
\end{array}\right]
$$

is the correlation matrix, with $\alpha=0.5$, a value to be confirmed with channel measurements.

\section{A.1.3 Next Generation Handheld Models}

The MIMO NGH channel models [124] characterize mobile and portable reception and extracted from a measurement that took place in Helsinki (Finland) 2010. These models were used during the DVB-NGH standardization process to evaluate performance of the MIMO schemes in realistic scenarios. Three scenarios are defined, outdoor mobile model, outdoor portable model, and an indoor portable model. While for the mobile case user velocities of $60 \mathrm{~km} / \mathrm{h}$ and $350 \mathrm{~km} / \mathrm{h}$ are defined, the portable case considers $3 \mathrm{~km} / \mathrm{h}$ and $0 \mathrm{~km} / \mathrm{h}$. As the MGM model, NGH models have a power delay profile of 8 taps and include $X$ factors and correlation between antennas $\mathbf{R}_{\mathbf{p}}$. However, NGH models have lower $K$ factors, higher $X$ factors (i.e., more coupling between polarizations) and higher covariance matrices $\mathbf{R}_{\mathbf{p}}$ than the MGM model.

Power delay profile Specific values of the power delay profile and $X$ factors for NGH portable/mobile outdoor (NGH-PO/NGH-mobile) and NGH portable indoor (NGH-PI) extracted from [17] are presented in Table A.2 and in Table A.3.

Table A.2: Power delay profile of the NGH portable/mobile outdoor channel model, extracted from $[17]$

\begin{tabular}{|c|c|c|c|}
\hline Tap numper, $p$ & Excess delay, $\tau_{p}(\mu s)$ & $\begin{array}{c}\text { Co-polar power gain } \\
\sigma_{11}^{2}\left(\tau_{p}\right)=\sigma_{22}^{2}\left(\tau_{p}\right) \mathrm{dB}\end{array}$ & $X_{j}$ \\
\hline $1 \mathrm{LoS}$ & 0 & -4.0 & 0.25 \\
\hline $2 \mathrm{NLoS}$ & 0.1094 & -7.5 & 0.25 \\
\hline $3 \mathrm{NLoS}$ & 0.2188 & -9.5 & 0.25 \\
\hline $4 \mathrm{NLoS}$ & 0.6094 & -11 & 0.25 \\
\hline $5 \mathrm{NLoS}$ & 1.109 & -15 & 0.25 \\
\hline $6 \mathrm{NLoS}$ & 2.109 & -26 & 0.25 \\
\hline $7 \mathrm{NLoS}$ & 4.109 & -30 & 0.25 \\
\hline $8 \mathrm{NLoS}$ & 8.109 & -30 & 0.25 \\
\hline
\end{tabular}


Table A.3: Power delay profile of the NGH portable indoor channel model, extracted from [17]

\begin{tabular}{|c|c|c|c|}
\hline Tap numper, $p$ & Excess delay, $\tau_{p}(\mu s)$ & $\begin{array}{c}\text { Co-polar power gain } \\
\sigma_{11}^{2}\left(\tau_{p}\right)=\sigma_{22}^{2}\left(\tau_{p}\right) \mathrm{dB}\end{array}$ & $X_{j}$ \\
\hline $1 \mathrm{LoS} / \mathrm{NLoS}$ & 0 & -6.0 & 0.56 \\
\hline $2 \mathrm{NLoS}$ & 0.1094 & -8.0 & 0.56 \\
\hline $3 \mathrm{NLoS}$ & 0.2188 & -10 & 0.56 \\
\hline $4 \mathrm{NLoS}$ & 0.6094 & -11 & 0.56 \\
\hline $5 \mathrm{NLoS}$ & 1.109 & -16 & 0.56 \\
\hline $6 \mathrm{NLoS}$ & 2.109 & -20 & 0.56 \\
\hline $7 \mathrm{NLoS}$ & 4.109 & -20 & 0.56 \\
\hline $8 \mathrm{NLoS}$ & 8.109 & -26 & 0.56 \\
\hline
\end{tabular}

For the NGH-PO and NGH-mobile the first tap is complete LoS component, while for the NGH-PI the first tap is Rice distributed - i.e., NLoS and LoS component - with factor $K=1$. The rest of the taps is the NGH models are NLoS with Rayleigh distribution. The model of the LoS term of the NGH channel models uses the expression in (A.4) and does not exhibit log-normal fading component as for the MGM channel.

Spatial correlation The spatial correlation for NGH-PO and NGH-mobile:

$$
\mathbf{R}_{p}=\sigma_{11}^{2}\left(\tau_{p}\right)\left[\begin{array}{cccc}
1 & .06 & .06 & .05 \\
.06 & .25 & .03 & .05 \\
.06 & .03 & .25 & .06 \\
.05 & .05 & .06 & 1
\end{array}\right]
$$

and the spatial correlation matrix for NGH-PI is:

$$
\mathbf{R}_{p}=\sigma_{11}^{2}\left(\tau_{p}\right)\left[\begin{array}{cccc}
1 & .15 & .1 & .15 \\
.15 & .56 & .06 & .04 \\
.10 & .06 & .56 & .15 \\
.15 & .04 & .15 & 1
\end{array}\right]
$$

As it can be observed by the previous spatial correlation matrices, NGH models is have a low degree of spatial correlation having higher correlation for NGH-PI model.

Doppler effect and power spectral density Generally, in mobile environments the channel realizations is a time variant function which depends on the 


\section{APPENDIX A. MIMO CHANNELS IN TERRESTRIAL BROADCASTING: MODELS AND CAPACITY}

relative speed of the transmit and receive pair. The time dependent variation of the channel realizations produce frequency shifts in the received signal known as Doppler effect. The maximum frequency shift $f_{D}$ in the received signal due to the Doppler effect is:

$$
f_{D}=\frac{v}{c} f_{c} \cos \alpha
$$

where $v$ is the receiver velocity ${ }^{1} c$ is the speed of light, $f_{c}$ is the carrier frequency of the signal, and $\alpha$ is the angle between directions of the receiver velocity and the arriving signal.

The pdf for Doppler frequency shifts depends on maximum Doppler frequency and the angular probability distribution of the received components. Under certain assumption such as signals propagate between the transmitter and the receiver in horizontal plane, the angles of arrival at the receive antenna are uniformly distributed, and a circular symmetric radiation pattern for the receive antenna, the power spectral distribution follows so-called Jakes power spectral density or the Clarke power spectral density. The Jakes power spectral density has the following expression:

$$
S\left(f, f_{d}\right)= \begin{cases}\frac{1}{\pi f_{d} \sqrt{1-\left(f / f_{D}\right)^{2}}} & \forall f \in]-f_{D}, f_{D}[ \\ 0 & \text { elsewhere }\end{cases}
$$

where the frequency shift components are distributed between $-f_{D}$, and $f_{D}$. A tap with Jakes power spectral density can be obtained by method of exact Doppler spread (MEDS) [125].

The tap spectral characteristic for the NGH-PO and NGH-mobile are:

$$
\begin{cases}S\left(f-3 f_{D} / 4, f_{D} / 4\right) & \text { for } p=2,3 \\ S\left(f+3 f_{D} / 4, f_{D} / 4\right) & \text { for } p=4,5,6,7,8\end{cases}
$$

where the tap $p=1$ is LoS with no Doppler shift and the tap spectral characteristics for the NGH-PI model are:

$$
\begin{cases}S\left(f, f_{D}\right) & \text { for } p=1 \\ S\left(f-3 f_{D} / 4, f_{D} / 4\right) & \text { for } p=2,3 \\ S\left(f+3 f_{D} / 4, f_{D} / 4\right) & \text { for } p=4,5,6,7,8\end{cases}
$$

where for tap $p=1$ the Doppler shifts are applied to the NLoS of the tap.

\footnotetext{
${ }^{1}$ The velocity $v$ corresponds to the relative velocity between the transmitter and receiver pair, where the transmitter and/or receiver can be in motion. The Doppler effect can be also observed in the received signal components from reflected objects that are in motion. In DTT systems the transmitters are normally static, therefore we assume in this thesis Doppler effect caused by mobile receivers.
} 
Rotation and imbalance matrices Additional matrices in the NGH models includes the effects of antenna alignment mismatch and transmit power imbalance in the NGH models.

The mismatch antenna matrix typically occurs in handset receivers where the orientation of the receive antenna depends on how the user holds the terminal. This mismatch is reduced in the case of rooftop reception since a professional installer aligns the receive antenna to the direction of the received signal. The mismatch matrix has the form of a rotation matrix:

$$
\mathbf{W}=\left[\begin{array}{cc}
\cos \Omega & -\sin \Omega \\
\sin \Omega & \cos \Omega
\end{array}\right]
$$

where $\Omega$ is the rotation angle and takes the values from the set $\{-45,0,+45\}$.

The imbalance matrix models the case where there exists a power imbalance between the two transmit vertical and horizontal polarizations. This situation is modelled with the diagonal matrix:

$$
\boldsymbol{\Gamma}=\left[\begin{array}{cc}
\Gamma_{11} & 0 \\
0 & \Gamma_{22}
\end{array}\right]
$$

where $\Gamma_{11}$ and $\Gamma_{22}$ can take the following values: $\left[\Gamma_{11}=1.1074, \Gamma_{22}=0.8796\right]$, $\left[\Gamma_{11}=1, \Gamma_{22}=1\right]$ and $\left[\Gamma_{11}=0.8796, \Gamma_{22}=1.1074\right]$. This transmit power imbalance model could also be included in the MGM channel model although it is not initially defined in the reference documents.

Signal model The basic signal model (without matrices $\boldsymbol{\Omega}$ and $\mathbf{W}$ ) for the NGH channels follow the expressions (A.7) and (A.8), but for the NGH-PO and NGH-mobile models the summation excludes the tap $p=1$ since the first tap is only LoS for those channels. When the matrices $\boldsymbol{\Omega}$ and $\mathbf{W}$ are included, expression (A.7) is replaced by:

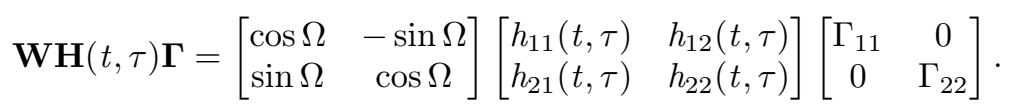

Due to the more static nature of the rotation and power imbalance matrices, they may affect multiple frames. The reinitialization of these variables may occur after various seconds, e.g., reference documents recommend five seconds.

\section{A.1.4 Extension to Four Transmit Antennas}

MIMO $4 \times 2$ channel models considered in thesis are composed at the transmitter side of 4 transmit cross-polar antennas (two horizontal and two vertical) and two cross-polar antennas at the receiver side. The case where the transmit antennas are co-located in the site is considered. The extension to 4 transmit 
antennas is achieved with two correlated independent instances of $2 \times 2$ MIMO channels previously described. For the second $2 \times 2$ MIMO NLoS and LoS components, the terms $\tilde{\mathbf{H}}_{w}$ and $\overline{\mathbf{H}}_{w}$ are replaced with $\dot{\mathbf{H}}_{w}$ and $\ddot{\mathbf{H}}_{w}$ where

$$
\begin{array}{r}
\operatorname{vec}\left(\dot{\mathbf{H}}_{w}\right)=\beta \operatorname{vec}\left(\tilde{\mathbf{H}}_{w}\right)+\sqrt{1-\beta^{2}} \operatorname{vec}\left(\hat{\mathbf{H}}_{w}\right), \\
\operatorname{vec}\left(\ddot{\mathbf{H}}_{w}\right)=\gamma \operatorname{vec}\left(\overline{\mathbf{H}}_{w}\right)+\sqrt{1-\gamma^{2}} \operatorname{vec}\left(\check{\mathbf{H}}_{w}\right)
\end{array}
$$

where $\hat{\mathbf{H}}_{w}$ and $\check{\mathbf{H}}_{w}$ are independent instances of i.i.d zero-mean complex Gaussian random matrices. The MGM model in [123] suggests a $\beta=0.5$ value for the NLOS. Chapter 3 of this thesis studies different correlation values $\gamma$ for the LOS in the $[0,1]$ range. Although the correlation between channel components from different polarizations is low [121], higher correlation values are observed between channel components with the same polarization [126]. Furthermore, strong LOS scenarios produces high correlated channels components [127, 128]. Specific values need to be confirmed with data extracted from measurement campaigns of cross-polar $4 \times 2 \mathrm{MIMO}$ in the UHF band.

\section{A.2 Ergodic Capacity with Asymptotic SNR}

For the performance analysis of the MIMO gains over SISO it will be convenient to use approximate ergodic capacity expressions in the high and low SNR regime.

Low SNR regime At low SNR values the ergodic capacity expression can approximated by [13]:

$$
\begin{array}{r}
C=\mathbb{E}\left\{\log \operatorname{det}\left(\mathbf{I}_{N_{r}}+\frac{\mathrm{SNR}}{N_{t}} \mathbf{H H}^{\dagger}\right)\right\}=\mathbb{E}\left\{\sum_{i=1}^{n_{\text {min }}} \log \left(\frac{\mathrm{SNR}}{N_{t}} \lambda_{i}^{2}\right)\right\} \\
\approx \mathbb{E}\left\{\sum_{i=1}^{n_{\text {min }}} \frac{\mathrm{SNR}}{N_{t}} \lambda_{i}^{2} \log (e)\right\}=\frac{\mathrm{SNR}}{N_{t}} \log (e) \sum_{i=1}^{n_{\text {min }}} \mathbb{E}\left\{\lambda_{i}^{2}\right\} \\
=\frac{\mathrm{SNR}}{N_{t}} \log (e) \mathbb{E}\left\{\|\mathbf{H}\|_{F}^{2}\right\}=\frac{\mathrm{SNR}}{N_{t}} \log (e) \mathbb{E}\left\{\sum_{i, j}^{N_{r}, N_{t}}\left|h_{i j}\right|^{2}\right\} .
\end{array}
$$

where $n_{\text {min }}$ is the minimum number of $N_{t}$ transmit and $N_{r}$ receive antennas in a i.i.d. Rayleigh channel with no spatial correlation and using the following approximation: $\log (1+x) \approx x \log (e)$ when $x \rightarrow 0$. 
High SNR regime At the high SNR regime the ergodic capacity can be approximated as [13]:

$$
\begin{array}{r}
C=\mathbb{E}\left\{\sum_{i=1}^{n_{\min }} \log \left(\frac{\mathrm{SNR}}{N_{t}} \lambda_{i}^{2}\right)\right\} \approx \mathbb{E}\left\{\sum_{i=1}^{n_{\min }} \log \frac{\mathrm{SNR}}{N_{t}} \lambda_{i}^{2}\right\} \\
=n_{\min } \log \mathrm{SNR}-n_{\min } \log N_{t}+\sum_{i=1}^{n_{\min }} \mathbb{E}\left\{\log \lambda_{i}^{2}\right\} \\
\approx n_{\min } \log \mathrm{SNR}
\end{array}
$$

where the following approximation: $\log (1+x) \approx \log (x)$ when $x \gg 1$ has been used.

\section{A.3 Performance Evaluation}

\section{A.3.1 Performance evaluation in basic channels}

Co-polar MIMO ergodic capacity without spatial correlation Fig. A.1 presents the ergodic capacity results under de i.i.d. Rayleigh channel with fast fading, $\mathrm{XPD}=1$ (co-polar antennas arrangement) and no correlation between antennas. This channel is described in section A.1.1. At the top, the figure shows ergodic capacity in bits/s/Hz vs. the SNR, and at the bottom it shows the gain ratio between the MIMO schemes over SISO, i.e., $C_{\mathrm{MIMO}} / C_{\mathrm{SISO}}$. As it can be observed at the top figure, the use of multiple receive antennas (SIMO) improves the SISO performance at all SNRs due to diversity and array gains. Compared to SISO, the SIMO capacity curve is shifted towards lower SNR values but maintains the same slope as the SISO curve. On the other hand, increasing only the number of transmit antennas to two (curved labelled with MISO $2 \times 1$ ) has the same performance as SISO at low SNRs but improves slightly the performance at higher SNRs. It also has the same slope as SISO/SIMO schemes. A significant improvement can be observed in the case of multiple antennas at the transmitter and receiver side (curve labelled with MIMO $2 \times 2$ ). Here, the slope of the capacity curve changes providing an increasing gain with increasing SNR. This change in the slope is due to the additional multiplexing gain. Further increasing the number of transmit antennas to four, provides and additional improvement over MIMO $2 \times 2$ but maintaining the same slope.

Additional channel state information at the transmitter (CTSI) makes, as expected, the performance of MISO $2 \times 1$ exactly equal to SIMO. On the other hand for MIMO $2 \times 2$ one can observe an improvement at low SNR which converges to the performance of MIMO $2 \times 2$ with CRSI only. For the MIMO $4 \times 2$ improvement is achieved at all SNR values compared to the unprecoded 
APPENDIX A. MIMO CHANNELS IN TERRESTRIAL BROADCASTING: MODELS AND CAPACITY
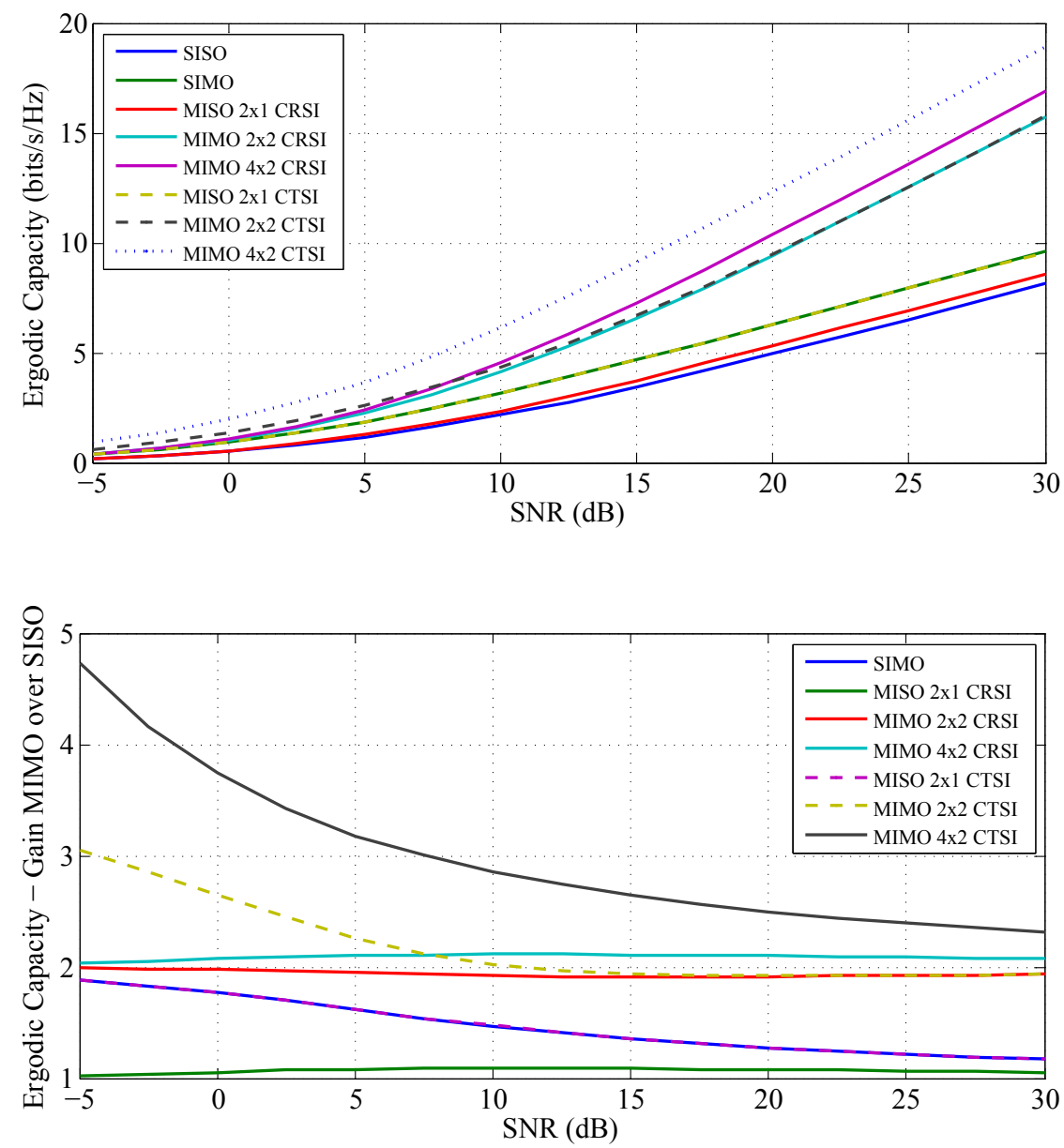

Figure A.1: i.i.d. Rayleigh fading with $\mathrm{XPD}=1$ and no correlation between antennas. Ergodic capacity vs. the SNR at the top and MIMO gain at the bottom.

case. While the gains for MIMO systems with the same number of transmit and receive antennas converges to an unprecoded system as the SNR increase, for MIMO systems with more transmit than receive antennas the gains is achieved at all SNRs [103].

The bottom of figure Fig. A.1 shows the gain ratio between the the ergodic capacity achieved with multi-antenna schemes and the ergodic capacity 
Table A.4: Summary ergodic MIMO capacity gains over ergodic SISO capaity in the high and low SNR limits for i.i.d. Rayleigh channel.

\begin{tabular}{|c|c|c|c|c|c|c|c|c|}
\cline { 4 - 9 } \multicolumn{1}{c|}{} & \multirow{2}{*}{$N_{t}$} & \multirow{2}{*}{$N_{r}$} & \multicolumn{2}{c|}{$\|\mathbf{H}\|_{F}^{2}$} & \multicolumn{2}{c|}{$C_{\text {MIMO }} / C_{\text {SISO }}$ when SNR $\rightarrow 0$} & \multicolumn{2}{c|}{$C_{\text {MIMO }} / C_{\text {SISO }}$ when SNR $\gg 1$} \\
\cline { 4 - 9 } & & & $\mathrm{XPD}=1$ & $\mathrm{XPD}=\infty$ & $\mathrm{XPD}=1$ & $\mathrm{XPD}=\infty$ & $\mathrm{XPD}=1$ & $\mathrm{XPD}=\infty$ \\
\hline $\mathrm{SISO}$ & 1 & 1 & 0.5 & 1.0 & - & - & - & - \\
\hline $\mathrm{SIMO}$ & 1 & 2 & 1.0 & 1.0 & 2.0 & 1.0 & 1.0 & 1.0 \\
\hline $\mathrm{MISO}_{2 \times 1}$ & 2 & 1 & 1.0 & 1.0 & 1.0 & 0.5 & 2.0 & 2.0 \\
\hline $\mathrm{MIMO}_{2 \times 2}$ & 2 & 2 & 2.0 & 2.0 & 2.0 & 1.0 & 2.0 & 2.0 \\
\hline $\mathrm{MIMO}_{4 \times 2}$ & 4 & 2 & 4.0 & 4.0 & 2.0 & 1.0 & & \\
\hline
\end{tabular}

achieved with SISO. SIMO multiplies the SISO ergodic capacity by a factor 2 at low SNRs but the gain decreases towards a factor 1 at increasing SNR. MIMO $2 \times 2$ on the other hand doubles the ergodic SISO capacity at all SNRs. At low SNR values, the gain comes from the fact of using multiple receive antennas, while the gain at high SNR values comes from the multiplexing gain by using multiple antennas at both the transmitter and receiver. Similarly to previous case, MIMO $4 \times 2$ doubles the ergodic SISO capacity at all SNR values, although we can observe an additional improvement compared to MIMO $2 \times 2$. The MIMO gains over SISO in the high and low SNR limits for i.i.d. Rayleigh channel are summarized in Table A.4. Here, one can observe how the approximate ergodic capacity values for CRSI at the high and low SNR regimes agree with the simulation results of the bottom of Fig. A.1.

Additional information about the channel at the transmitter (i.e., curves labelled with CTSI) makes the performance of MISO $2 \times 1$ equal to SIMO. At low SNR, $2 \times 2$ and $4 \times 2$ MIMO schemes multiply the SISO ergodic capacity by up to 3 and 4.5 respectively. At higher SNR values, although the performance of MIMO $2 \times 2$ with CTSI approaches the performance with CRSI only, MIMO $4 \times 2$ with CTSI shows an improvement compared with CRSI only at all SNRs.

Co-polar MIMO outage capacity without spatial correlation Fig. A.2 presents the same analysis as in Fig. A.1 but for 5\% outage capacity. With outage capacity the gains over SISO are higher at all SNRs. In this channel with uncorrelated antenna paths, increasing the number of antennas reduces significantly the probability that the channel is in outage.

Co-polar MIMO gains with spatial correlation Fig. A.3 shows the effect of antenna correlation in the ergodic and outage capacity of a i.i.d Rayleigh channel with $\mathrm{XPD}=1$. High and low correlation between antennas is included in the figure to study the effects of correlation in the MIMO capacity. In the case of low correlation the matrix $\mathbf{R}=\mathbf{I}$, i.e., no correlation between antennas. For the case of high correlation the matrix $\mathbf{R}=\mathbf{R}_{\text {High }}$ where the matrix $\mathbf{R}_{\text {High }}$, 
APPENDIX A. MIMO CHANNELS IN TERRESTRIAL BROADCASTING: MODELS AND CAPACITY
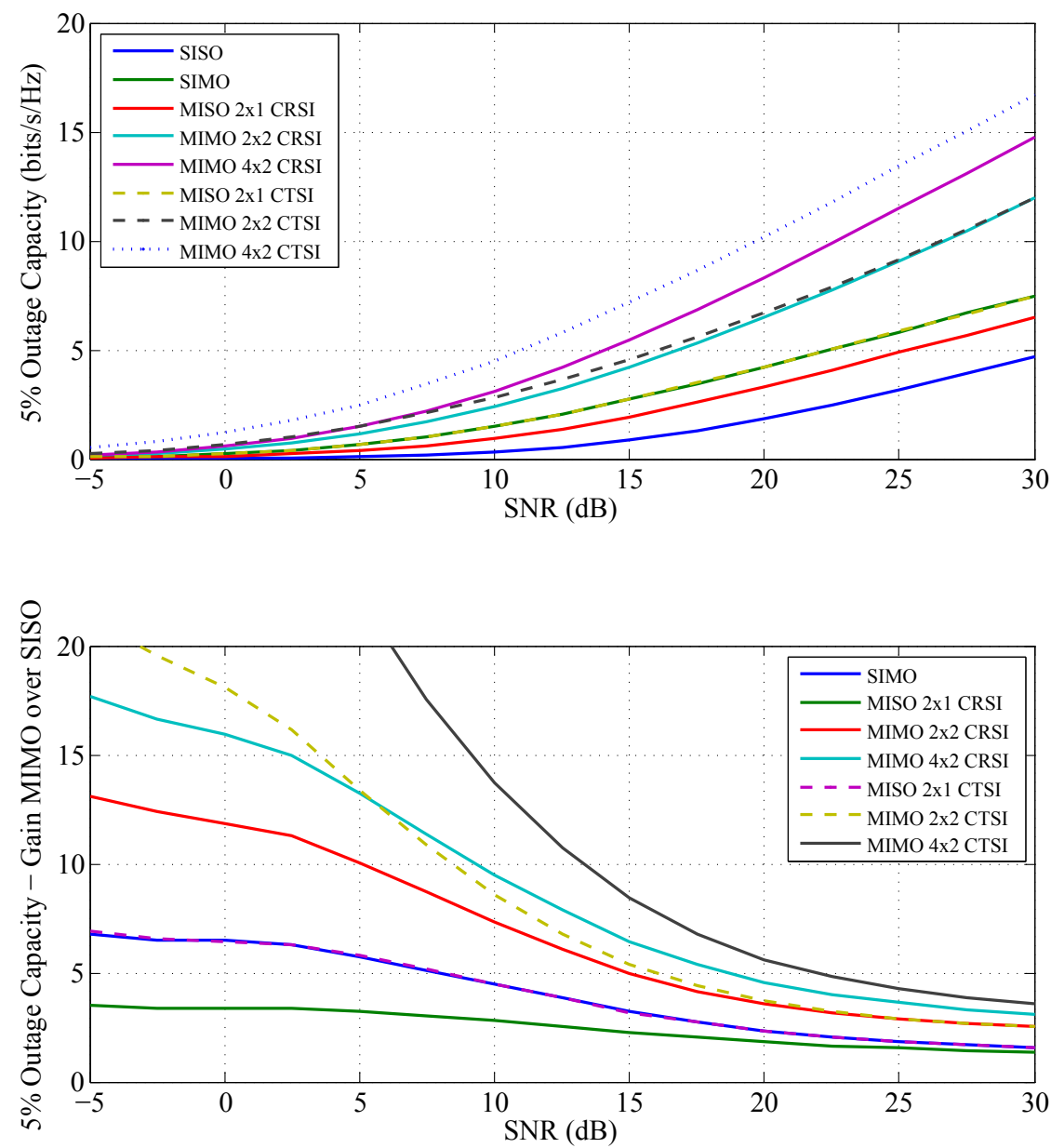

Figure A.2: i.i.d. Rayleigh fading with $\mathrm{XPD}=1$ and no correlation between antennas. $5 \%$ outage capacity vs. the SNR at the top and MIMO gain at the bottom.

extracted from reference [129] has the following coefficients:

$$
\mathbf{R}_{\text {High }}=\left[\begin{array}{cccc}
1 & .9 & .9 & .81 \\
.9 & 1 & .81 & .9 \\
.9 & .81 & 1 & .9 \\
.81 & .9 & .9 & 1
\end{array}\right]
$$



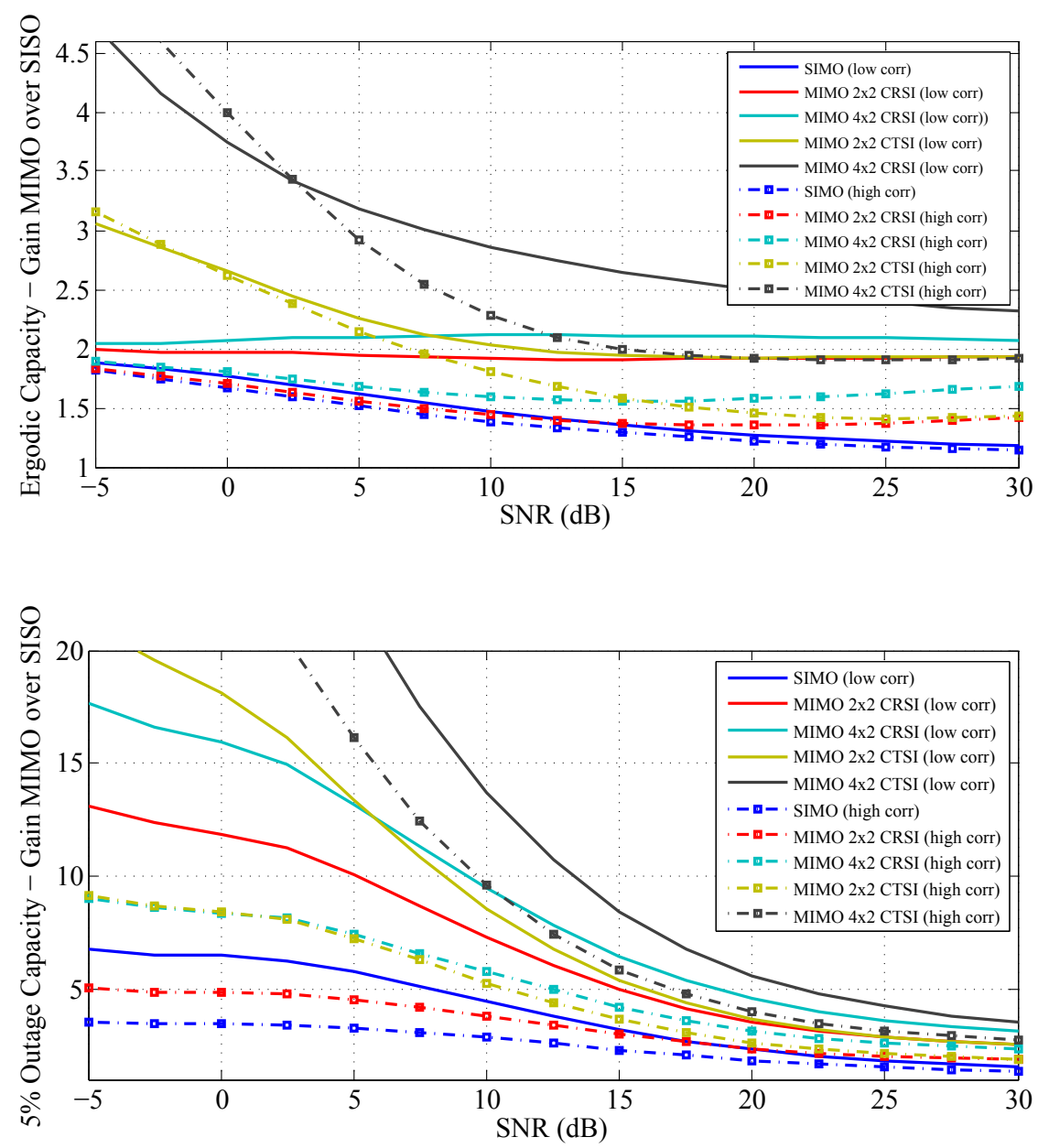

Figure A.3: i.i.d. Rayleigh fading with $\mathrm{XPD}=1$, and with two types of correlation between antennas: low correlation (i.e., $\mathbf{R}=\mathbf{I}$ ) and high correlation (i.e., $\mathbf{R}=\mathbf{R}_{\text {High }}$ ). MIMO gain vs. SNR with ergodic capacity at the top and $5 \%$ outage capacity at the bottom.

For the ergodic capacity results at the top of Fig. A.3 correlation reduces the MIMO gain for all the antenna schemes. This reduction is more significant for the schemes that have multiplexing gain, as discussed in section 2.2. A MIMO channel matrix with correlated components makes the channel matrix 


\section{APPENDIX A. MIMO CHANNELS IN TERRESTRIAL BROADCASTING: MODELS AND CAPACITY}

singular which decreases the multiplexing gain. For SIMO, only a marginal reduction is observed since the power gain is retained and only the diversity gain is lost due to correlated fading. With CTSI, same performance loss is observed at high SNRs. However, at low SNRs, higher ergodic capacity can be achieved with correlated antennas than with un-correlated antennas. Similar results is observed in references [103, 96].

For the $5 \%$ outage capacity results at the bottom of Fig. A.3 presents similar behaviour to the ergodic capacity plot, i.e., the MIMO gain reduces due to correlated antennas. However, with CTSI also reduces the available gain at the low SNR regime. Here, the loss of SIMO is also significant, specially at low SNRs. With correlated antennas the probability that all channel paths are in outages increases, reducing the outage capacity.

Co-polar vs. Cross-polar MIMO with spatial correlation Fig. A.4 shows the same results as in Fig. A.3 but for high spatial correlation and two types XPD values, $\mathrm{XPD}=1$ (full inter-antenna interference - co-polar antennas) and $\mathrm{XPD}=\infty$ (no inter-antenna interference - perfect cross-polar antennas). First the ergodic capacity results at the top of Fig. A.4 for the MIMO schemes with $\mathrm{XPD}=\infty$ are studied. With cross-polar antennas, SIMO provides the same performance than SISO at all SNRs, and MISO $2 \times 1$ provides a performance loss compared to SISO at low SNRs (towards a 0.5 ratio) but this loss is reduced with increasing SNR towards a value of 1 . On the other hand, the gains of $2 \times 2$ and $4 \times 2$ MIMO schemes tend to 1 at low SNRs, but increase with increasing SNR asymptotically to a gain of 2 . These gains can also be predicted by expressions (A.21) and (A.22) and summarized in Table A.4. The performance of MIMO schemes with XPD $=\infty$ compares with the case of XPD $=1$ in the following way. For SIMO, the gains achieved with XPD $=1$ are higher than $\mathrm{XPD}=\infty$ at all SNRs. The same comparison can be done for MISO $2 \times 1$, but here $\mathrm{XPD}=\infty$ always performs worse than SISO, that discourages its use. In the case of $2 \times 2$ and $4 \times 2 \mathrm{MIMO}$, although $\mathrm{XPD}=\infty$ does not provide a benefit compared to XPD $=1$ at low SNRs, it can overcome the performance degradation due to spatial correlation at high SNRs.

In terms of outage capacity results at the bottom of Fig. A.4 for all the schemes, $\mathrm{XPD}=\infty$ always provides lower or equal performance than $\mathrm{XPD}=1$ at all SNRs. This performance difference is specially significant at low SNR values. It is interesting to note that for $2 \times 2$ and $4 \times 2$ MIMO with either $\mathrm{XPD}=1$ or $\mathrm{XPD}=\infty$ reach the same value at high SNRs. This is not the case for SIMO and MISO $2 \times 1$ with $\mathrm{XPD}=1$ which still obtain higher gain than $\mathrm{XPD}=\infty$ at high $\mathrm{SNR}$ values. 

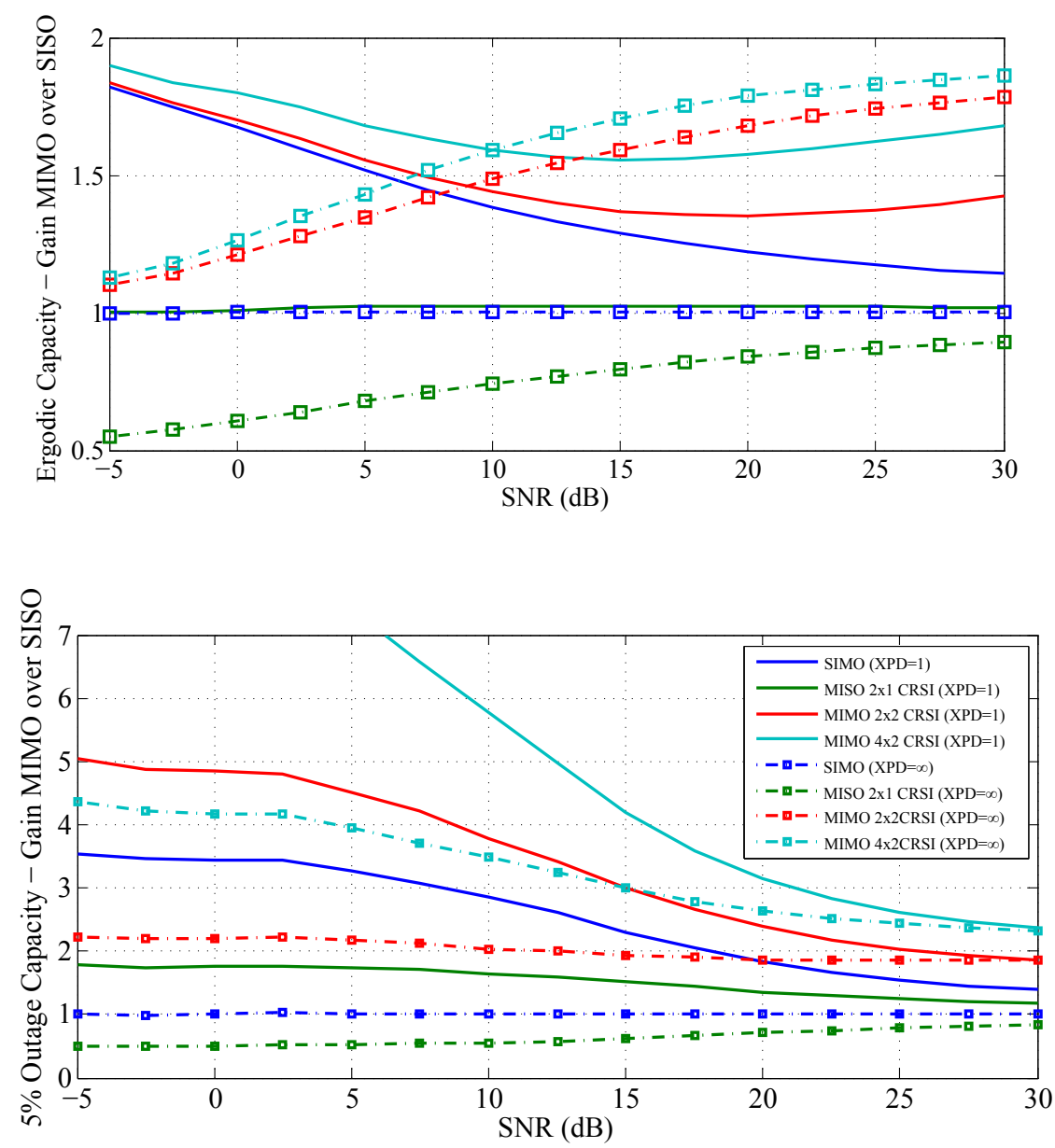

Figure A.4: i.i.d. Rayleigh fading with high correlation (i.e., $\mathbf{R}=\mathbf{R}_{\text {High }}$ ) with $\mathrm{XPD}=1$ (co-polar) and $\mathrm{XPD}=\infty$ (cross-polar). MIMO gain vs. SNR with ergodic capacity at the top and $5 \%$ outage capacity at the bottom.

\section{A.3.2 Performance evaluation in Terrestrial broadcast chan- nels}

Outage Capacity of MGM channel model Fig. A.5 presents for the MGM channel described in section A.1.2 the $5 \%$ outage capacity the capacity 


\section{APPENDIX A. MIMO CHANNELS IN TERRESTRIAL BROADCASTING: MODELS AND CAPACITY}

vs. the SNR at the top, and the MIMO gain at the bottom, i.e., $C_{\mathrm{MIMO}} / C_{\mathrm{SISO}}$. It includes the same MIMO schemes as in previous figures but discard MISO $2 \times 1$ due to the poor performance in cross-polar channels. Since the channel realizations of the MGM channel are block fading type, outage capacity metric is selected. Due to cross-polar nature of the MGM channel with low interantenna interference the gain of SIMO over SISO is low. On the other hand MIMO $2 \times 2$ due to multiplexing gain provides an increasing improvement at increasing SNRs. Similarly MIMO $4 \times 2$ also exploits the multiplexing gain but an additional diversity is exploit due to the increased number or transmit antennas which provide and additional improvement.

As in previous analysis additional CTSI introduces a gain at low SNRs for MIMO $2 \times 2$ an also introduces a gain at high SNRs for MIMO $4 \times 2$.

The bottom of Fig. A.5 shows the corresponding MIMO gains of the schemes at the top of the same figure. The MIMO gains at some representative SNRs are summarized in Table A.5.

Due to the log-normal fading term of the MGM channel, the mean SNRs in Fig. A.5 are $9 \mathrm{~dB}$ higher than the required SNR for the system modulation and coding [15]. For instance, SNR values of 24 and $27 \mathrm{~dB}$ correspond to SNR thresholds of $15 \mathrm{~dB}$ and $18 \mathrm{~dB}$, respectively.

Table A.5 shows the MIMO gains at some representative SNR values: 4, 9, 27 and $30 \mathrm{~dB}$ which correspond to $-5,0,18$ and $21 \mathrm{~dB}$ without the lognormal fading term. Due to received signal strength levels at rooftop reception antennas, mean SNR values of $20 \mathrm{~dB}$ are realistic [17]. At $30 \mathrm{dBs}$ (i..e, $21 \mathrm{~dB}$ mean SNR $+9 \mathrm{~dB} d$ ) as presented in Table A.5 SIMO can only multiply the SISO capacity by 1.1. Implementing multiple antennas at the transmitter (and without CTSI) the SISO gain can be multiplied by 2 and 2.4 with $2 \times 2$ and $4 \times 2$ MIMO respectively. With additional CTSI, the same gain is obtained for $2 \times 2$ MIMO and a 2.7 gain factor for $4 \times 2$ MIMO. In this rooftop reception scenario, SIMO only provides gains compared to SISO between 10\% (at high SNRs) and $40 \%$ (at low SNRs), which may not justify the economical cost carried by the upgrade of the rooftop antennas of the receiving population. However, multiple transmit and receive antennas at least doubles the capacity in all cases of Table A.5, which may be justify the economical expenses carried by the upgrade in the transmitters and receivers.

Outage Capacity of NGH-PI channel model Fig. A.6 presents the same analysis as in the Fig. A.5 but for the NGH-PI channel described in section A.1.3. Due to the block fading nature of portable channels, here for the NGH-PI channel outage capacity is also selected as representative metric. At the top of Fig. A.6 it can be observed higher gains of the MIMO schemes over SISO than for the MGM channel. Specially for SIMO, the gains are visible higher at all SNRs. The gain of MIMO schemes over SISO is represented at 


\section{A.3 Performance Evaluation}
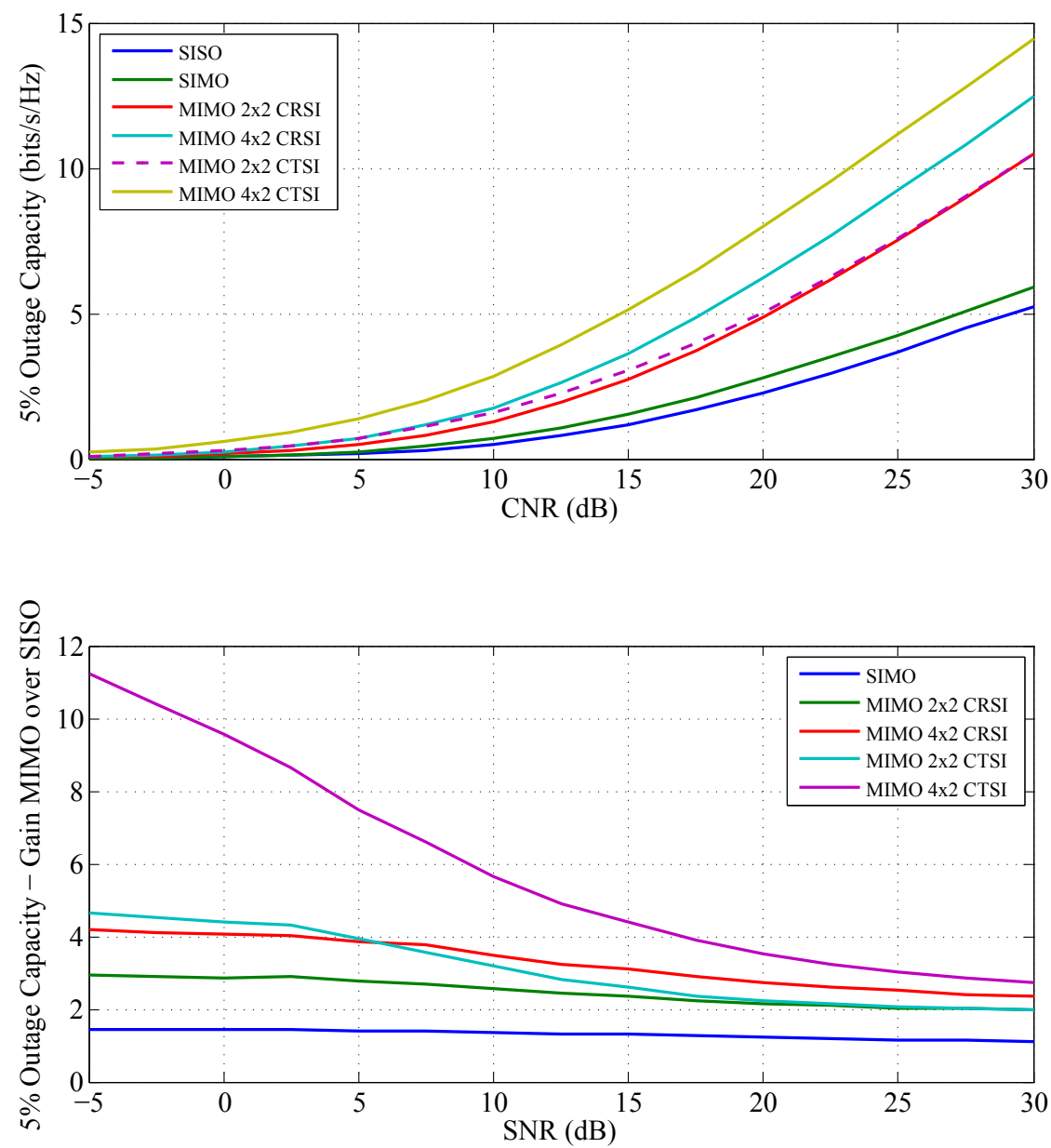

Figure A.5: Outage capacity at the $5 \%$ outage probability for the MGM channel. $5 \%$ outage capacity vs. the SNR at the top, and MIMO gains vs. the SNR at the bottom.

the bottom of Fig. A.6 and a summary table in some representative SNR values is presented in Table A.6. Assuming signal strength levels for a network designed for fixed rooftop reception (antenna height $10 \mathrm{~m}$ ), the SNR for a receiver in an indoor environment can attain values around $-4 \mathrm{~dB}$ [17]. With a receive SNR of $-5 \mathrm{~dB}$ as presented in Table A.6 SIMO multiplies the SISO by a factor of 3.2 , MIMO $2 \times 2$ by a factor of 4.4 and MIMO $4 \times 2$ by a factor of 
Table A.5: $5 \%$ outage capacity MIMO gains over SISO in MGM channel for representative SNRs.

\begin{tabular}{|c|c|c|c|c|c|}
\hline \multirow{2}{*}{ SNR \Scheme } & \multirow{2}{*}{ SIMO } & \multicolumn{2}{|c|}{ MIMO $2 \times 2$} & \multicolumn{2}{c|}{ MIMO $4 \times 2$} \\
\cline { 3 - 6 } & & CRSI & CTSI & CRSI & CTSI \\
\hline $4 \mathrm{~dB}$ & 1.4 & 2.8 & 4.1 & 3.9 & 7.9 \\
\hline $9 \mathrm{~dB}$ & 1.4 & 2.7 & 3.3 & 3.6 & 6.0 \\
\hline $27 \mathrm{~dB}$ & 1.1 & 2.0 & 2.0 & 2.43 & 2.9 \\
\hline $30 \mathrm{~dB}$ & 1.1 & 2.0 & 2.0 & 2.4 & 2.7 \\
\hline
\end{tabular}

5.0. Additional CTSI increases these factors to 7.0 and to 12.2 for $2 \times 2$ and $4 \times 2$ MIMO, respectively. Gains in the same order are achieved for a SNR of $0 \mathrm{~dB}$ (without CTSI).

Table A.6: 5\% outage capacity MIMO gains over SISO in NGH-PI channel for representative SNRs.

\begin{tabular}{|c|c|c|c|c|c|}
\hline \multirow{2}{*}{ SNR $\backslash$ Scheme } & \multirow{2}{*}{ SIMO } & \multicolumn{2}{|c|}{ MIMO $2 \times 2$} & \multicolumn{2}{c|}{ MIMO $4 \times 2$} \\
\cline { 3 - 6 } & & CRSI & CTSI & CRSI & CTSI \\
\hline$-5 \mathrm{~dB}$ & 3.2 & 4.4 & 7.0 & 5.0 & 12.2 \\
\hline $0 \mathrm{~dB}$ & 3.0 & 4.0 & 5.8 & 4.7 & 8.9 \\
\hline $18 \mathrm{~dB}$ & 1.6 & 2.5 & 2.4 & 2.9 & 3.6 \\
\hline $21 \mathrm{~dB}$ & 1.5 & 2.4 & 2.4 & 2.8 & 3.32 \\
\hline
\end{tabular}

Outage Capacity of NGH-PO channel model Fig. A.7 presents the same analysis as in the Fig. A.6 but for the NGH-PO channel described in section A.1.3. As for the NGH-PI channel outage capacity as representative metric for portable channels is selected. Overall, it is noted that similar tendency for the MIMO schemes in the NGH-PO as in the NGH-PI channel at the top of Fig. A.7 . However, lower gains are achieved this channel as presented at the bottom of the Fig. A.7. A summary of table of the MIMO gains over SISO in some representative SNR values is presented in Table A.7. Following a similar argument as for the NGH-PI channel analysis, the received SNR for a terminal in an outdoor environment in a network planned for fixed rooftop reception, can reach values around $16 \mathrm{~dB}$ [17]. With a receive SNR of $18 \mathrm{~dB}$ as presented in Table A.7 SIMO multiplies the SISO by a factor of 1.4, MIMO $2 \times 2$ by a factor of 2.2 and MIMO $4 \times 2$ by a factor of 2.4. Additional CTSI does not provide additional case for MIMO $2 \times 2$ but increases the gain to a factor 3.0 for MIMO $4 \times 2$. 

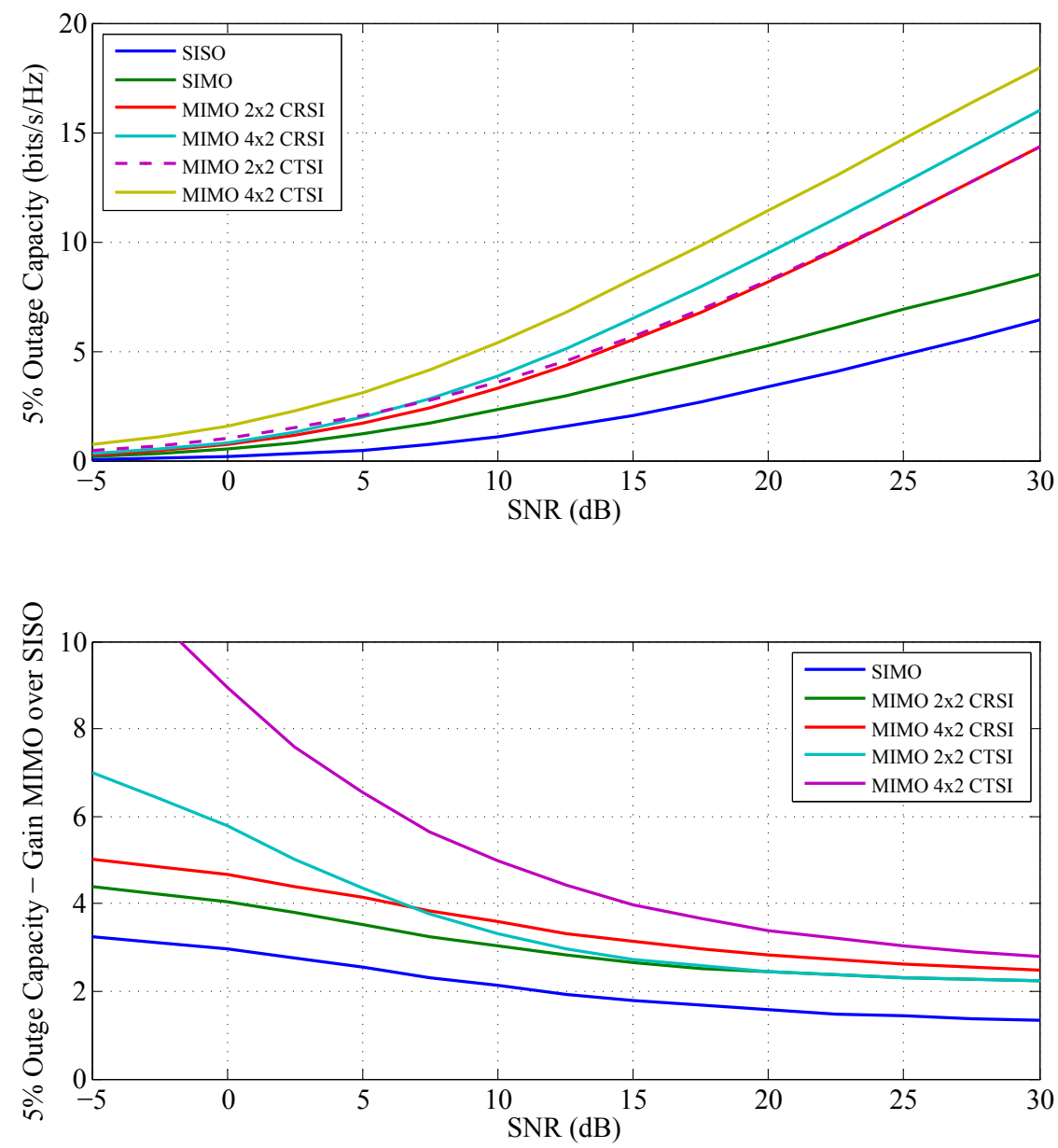

Figure A.6: Outage capacity at the $5 \%$ outage probability for the NGH-PI channel. $5 \%$ outage capacity vs. the SNR at the top, and MIMO gains vs. the SNR at the bottom.

A closer look at the MIMO gains presented in Table A.6 and Table A.7 shows that the gains are higher for NGH-PI channel. Although both channels include rotation and imbalance matrices described in section A.1.3 and share other similarities such as the delay spread of the power delay profile, differ in other characteristics such as: amplitudes of the power delay profile, distribution of the first tap (LoS for NGH-PO and NLoS+LoS for NGH-PI), and the XPD 
factor. The $X$ factor for the NGH-PI channel is lower than the $X$ factor of the NGH-PO channel (higher coupling between polarizations for the NGH$\mathrm{PI}$ ). This has a direct impact in the performance results with outage capacity, since MIMO cross-polar channels with low $X$ factors (low coupling between polarizations) are detrimental for spatial diversity [130]. Since low $X$ factor reduce the number of effective channel paths, it increases the outage probability that all channel paths are in deep fade.

Table A.7: $5 \%$ outage capacity MIMO gains over SISO in NGH-PO channel for representative SNRs.

\begin{tabular}{|c|c|c|c|c|c|}
\hline \multirow{2}{*}{ SNR $\backslash$ Scheme } & \multirow{2}{*}{ SIMO } & \multicolumn{2}{|c|}{ MIMO $2 \times 2$} & \multicolumn{2}{c|}{ MIMO $4 \times 2$} \\
\cline { 3 - 6 } & & CRSI & CTSI & CRSI & CTSI \\
\hline$-5 \mathrm{~dB}$ & 2.3 & 3.0 & 4.5 & 3.2 & 7.2 \\
\hline $0 \mathrm{~dB}$ & 2.1 & 2.8 & 3.7 & 3.1 & 5.6 \\
\hline $18 \mathrm{~dB}$ & 1.4 & 2.2 & 2.2 & 2.4 & 3.0 \\
\hline $21 \mathrm{~dB}$ & 1.3 & 2.1 & 2.1 & 2.4 & 2.8 \\
\hline
\end{tabular}

Ergodic Capacity of NGH-mobile channel model Fig. A.8 presents for the NGH-mobile channel described in section A.1.3 the ergodic capacity vs. the SNR at the top, and the MIMO gain at the bottom. The same MIMO schemes as in previous figures are included. In this case ergodic capacity is selected as representative measure, since within a code word the channel realizations vary due to the motion of the receiver terminals. The MIMO gains at the bottom of Fig. A.8 show a behaviour between the bottom of Fig. A.1 and the top of Fig. A.4. The MIMO gains in the NGH-mobile channel are shown at the bottom of Fig. A.8 and a summary of some representative SNRs is given in Table A.15. As for the NGH-PO channel, realistic received SNR values for a vehicular terminal in a broadcast network designed for rooftop reception can take values around $18 \mathrm{~dB}$. In this case the SIMO multiplies the SISO by a factor of 1.2 , MIMO $2 \times 2$ by a factor of 1.8 and MIMO $4 \times 2$ by a factor of 2.0. Additional CTSI provides almost no additional gain for MIMO $2 \times 2$ but increases the gain to a factor 2.4 for MIMO $4 \times 2$. 


\section{A.3 Performance Evaluation}
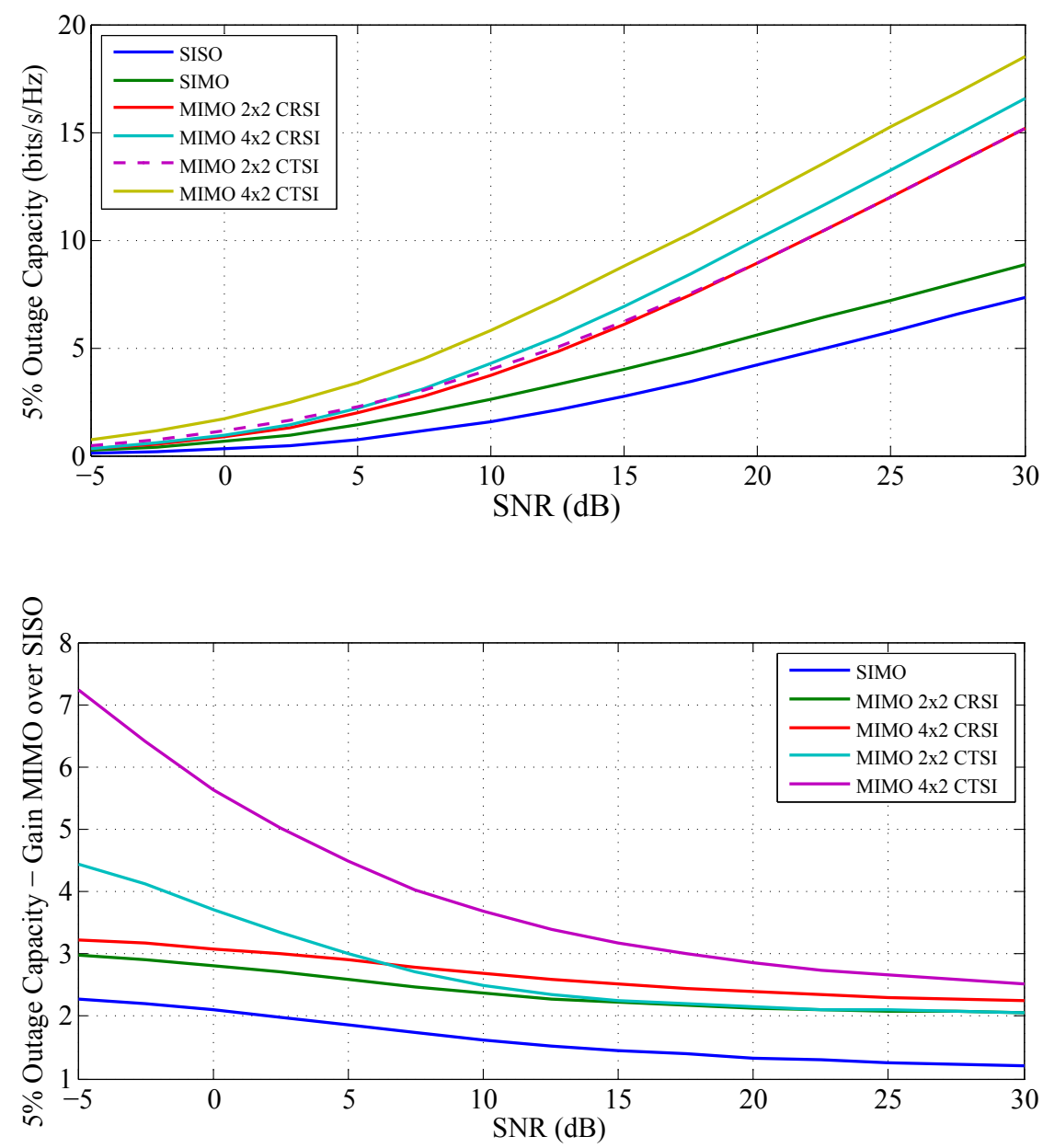

Figure A.7: Outage capacity at the $5 \%$ outage probability for the NGH-PO channel. $5 \%$ outage capacity vs. the SNR at the top, and MIMO gains vs. the SNR at the bottom. 
APPENDIX A. MIMO CHANNELS IN TERRESTRIAL BROADCASTING: MODELS AND CAPACITY
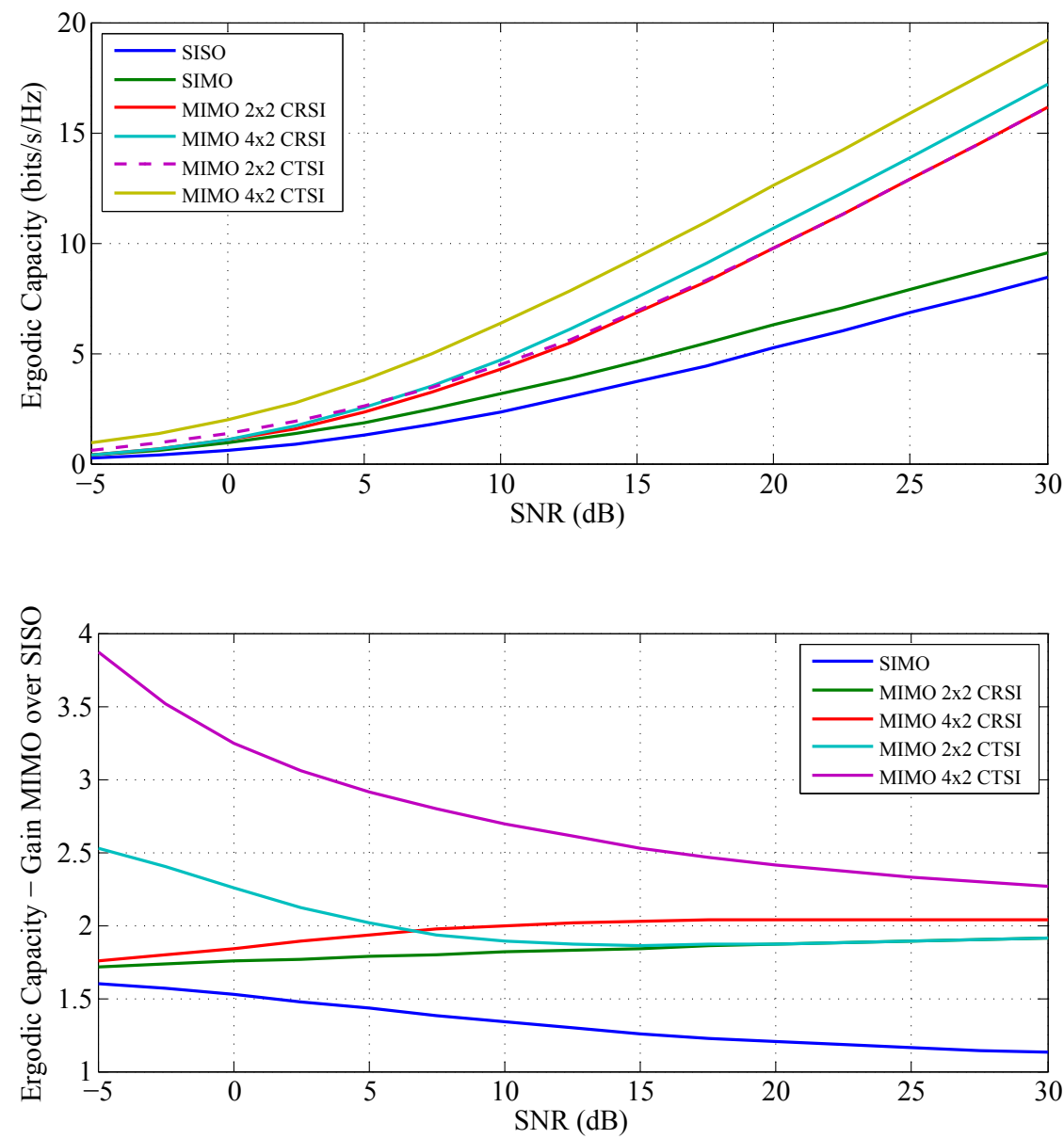

Figure A.8: Ergodic capacity for the NGH-mobile channel.Ergodic capacity vs. the SNR at the top, and MIMO gains vs. the SNR at the bottom.

Table A.8: Ergodic capacity MIMO gains over SISO in NGH-mobile channel for representative SNRs.

\begin{tabular}{|c|c|c|c|c|c|}
\hline \multirow{2}{*}{ SNR $\backslash$ Scheme } & \multirow{2}{*}{ SIMO } & \multicolumn{2}{|c|}{ MIMO $2 \times 2$} & \multicolumn{2}{c|}{ MIMO $4 \times 2$} \\
\cline { 3 - 6 } & & CRSI & CTSI & CRSI & CTSI \\
\hline$-5 \mathrm{~dB}$ & 1.6 & 1.7 & 2.5 & 1.8 & 3.9 \\
\hline $0 \mathrm{~dB}$ & 1.5 & 1.7 & 2.3 & 1.8 & 3.2 \\
\hline $18 \mathrm{~dB}$ & 1.2 & 1.8 & 1.9 & 2.0 & 2.4 \\
\hline $21 \mathrm{~dB}$ & 1.2 & 1.9 & 1.9 & 2.0 & 2.4 \\
\hline
\end{tabular}




\section{Appendix B}

\section{DVB-NGH and ATSC 3.0 MIMO Physical Layers}

7 HIS APPENDIX presents the MIMO physical layers adopted for DVB-NGH 1 and ATSC 3.0 specifications. Both specifications share similar architecture for the implementation of MIMO. First, a common transmit block diagram for the two specifications is presented. Then, specific differences and commonalities for each of the transmitting blocks are detailed. 


\section{B.1 Generic Transmit MIMO DVB-NGH \& ATSC 3.0 Physical layer}

MIMO has been adopted in DVB-NGH and ATSC 3.0 specifications as optional technologies, i.e., its implementation is not mandatory for the transmitters and receivers.

The implementation of MIMO for the optional MIMO profile in DVB-NGH and ATSC 3.0 requires additional elements compared to the base profile with single transmit and receive antenna architecture such an extra cross-polarized antenna with the inferred hardware (i.e. cooling systems, RF feedings, power combiners, amplifiers, etc.), as well as a modified transmission chain. Fig. B.1 depicts basic building blocks of a transmit MIMO physical layer: forward error correction (FEC), bit-interleavers (BIL), bit demultiplexer to multiple streams (DEMUX), mapper (MAP), MIMO precoder, interleavers (IL) and framing, and waveform. Chapter 2 of this dissertation describes the wireless fundamentals for each of the transmitting blocks.

\section{B.2 Overview of DVB-NGH and ATSC 3.0 Trans- mitting blocks}

\section{Forward Error Correction}

Both DVB-NGH and ATSC 3.0 use FEC based on a concatenation of BCH codes and LDPC codes. Both specifications use the same LDPC codes as the ones adopted for the baseline SISO profiles. The LDPC codes included in ATSC 3.0 optimize the performance of the LDPC codes included in DVB-NGH and therefore provide better performance [78]. ATSC 3.0 specifies code sizes of 16200 bits (short codes) and 64800 bits (long codes), however for the MIMO profile the long code is only specified. DVB-NGH targeting mobile devices with limited hardware requirements specifies up to 16200 bits code size. The MIMO profile of DVB-NGH defines 7 code-rates from $5 / 15$ to $11 / 15$ with equal spacing. ATSC 3.0 widens this set with a total of 13 code-rates from $2 / 15$ to $13 / 15$.

\section{Bit-Interleavers}

In DVB-NGH the bit-interleaver, different from the one for the basic SISO profile, has been optimized for MIMO transmissions exploiting the quasi-cyclic structure of the LDPC codes. The bit-interleaver for MIMO consists of two components: a parity interleaver and a parallel bit interleaver. The parity interleaver is the same as for the base profile and the parallel interleaver in turn 


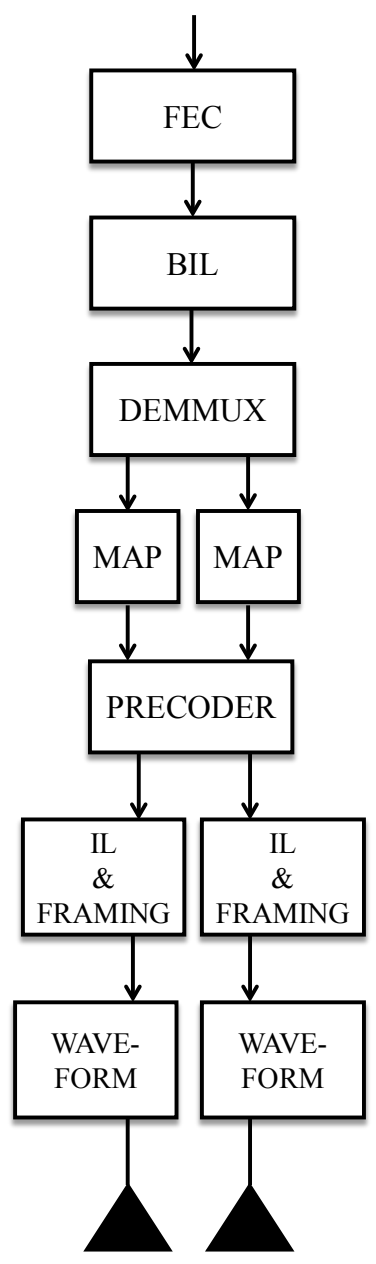

Figure B.1: Generic transmit MIMO block diagram for DVB-NGH and ATSC 3.0 specifications. 
Table B.1: Physical layer parameters comparison of DVB-NGH and ATSC 3.0 using MIMO

\begin{tabular}{|c|c|c|}
\hline PHY parameters & $D V B-N G H$ & $A T S C 3.0$ \\
\hline$N_{r} \times N_{t}$ & $2 \times 2$ & $2 \times 2$ \\
\hline LDPC code size & 16200 & 64800 \\
\hline bits per cell & 6,8 and 10 & $4,8,12,16,20,22$ \\
\hline Precoder & $\begin{array}{l}\text { Antenna power allocation } \\
\text { Rotation } \\
\text { Stream power allocation } \\
\text { Phase hopping }\end{array}$ & $\begin{array}{c}\text { Rotation } \\
\text { IQ interleaving } \\
\text { Phase Hopping }\end{array}$ \\
\hline
\end{tabular}

comprises two stages: an adjacent quasi-cyclic block interleaver and a section interleaver and presented in Fig. B.2. It exhibits a low complexity, low latency, and fully parallel design that ease the implementation of iterative structures which provide significant gains on the top of the significant MIMO gain. The quasi-cyclic block interleaving is based on permutation sequences that have been optimized for all combinations of bpc and transmit power imbalance. Deliberate transmit power imbalance of 6 and $9 \mathrm{~dB}$ is allowed in DVB-NGH to ease the integration of MIMO networks. When intentional transmit power imbalance is applied it provides a reasonable coverage reduction for single antenna terminals while MIMO precoders are optimized to maintain good performance in this situation. Furthermore, these permutations optimize the gain achieved by iterative decoding receivers.

ATSC 3.0 uses the same bit-interleaver structure as DVB-NGH but with the quasi-cyclic block interleaving permutations have been designed to optimize the performance of the SISO profile employing non-uniform constellations. MIMO in ATSC 3.0 reuses the bit-interleavers from the SISO baseline and have not been specifically optimized for MIMO transmissions. Additional transmission parameters are specified in Table B.2.

\section{Demultiplexers}

The antenna stream demultiplex distributes the output bits from the bitinterleaver into two constellation mappers, one for each transmit antenna. DVB-NGH applies a different permutation sequence for each of the bpc in Table B.2. In ATSC 3.0 the demultiplex permutation does not depend on the number of bpc, instead, the code-word stream is divided in groups of bpc bits and the first bpc/2 bits are mapped to the first transmit antenna, and the second bpc/2 bits are mapped to the second transmit antenna. 


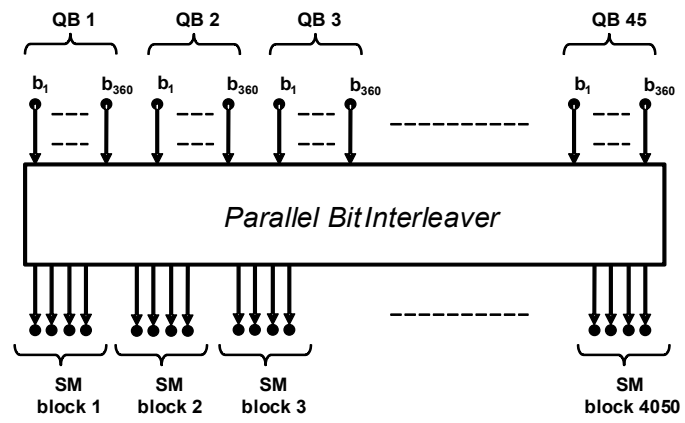

Figure B.2: Parallel Bit-Interleaver used in DVB-NGH.

\section{Mappers}

After the demultiplexer, the groups of bits in each antenna stream are mapped to complex constellation symbols (cf. Chapter 2).

Three steps of spectral efficiency are defined in DVB-NGH for MIMO (cf. Table B.2) with $6 \mathrm{bpc}, 8 \mathrm{bpc}$ and $10 \mathrm{bpc}$. Asymmetric constellations are employed for each transmit antenna to provide higher granularity. The defined constellation for the first/second antenna are QPSK+16QAM for the case of $6 \mathrm{bpc}, 16 \mathrm{QAM}+16 \mathrm{QAM}$ for the case of $8 \mathrm{bpc}$, and 16QAM+64QAM for the case of $10 \mathrm{bpc}$.

The MIMO scheme adopted in ATSC 3.0 re-uses the SISO antenna baseline constellations, and hence it introduces the use of MIMO with non-uniform constellations. In addition to QPSK, ATSC 3.0 has adopted two-dimensional non-uniform constellations for cardinalities of 16, 64 and 256 points, and onedimensional non-uniform constellations for cardinalities of 1024 and 4096 points [78]. No asymmetric constellations are employed in the MIMO scheme adopted in ATSC 3.0 since enough granularity in spectral efficiency is provided with all the combinations of bpc and available code-rates.

\section{Precoder}

The MIMO precoding adopted in DVB-NGH and ATSC 3.0 is described in detail in Chapter 3 in subsection 3.2.3 of this dissertation.

\section{Interleavers \& Framing}

Time interleaver In DVB-NGH the precoded constellation symbols of each antenna stream are time interleaved. The time interleaver is composed of two 


\section{APPENDIX B. DVB-NGH AND ATSC 3.0 MIMO PHYSICAL LAYERS}

stages, i.e. inter-frame convolutional interleaver and intra-frame block interleaver [47]. However, for MIMO the time interleaver process is implemented to each transmit layer. Due to the same memory constrains apply for all DVBNGH transmission schemes, i.e. MIMO and non-MIMO, the time interleaving depth for MIMO is halved compared to non-MIMO transmissions [44].

In ATSC 3.0 for single physical layer pipe mode a sheer convolutional time interleaving is used, whereas for the multiple physical layer pipe mode a hybrid time interleaving composed of cell interleaver, twisted block interleaver, and a convolutional delay-line is used. Optionally, the convolutional time interleaving and the hybrid time interleaving can be used in conjunction with extended time interleaving and a cell interleaver (only for hybrid time interleaving) to further improve robustness over long burst error lengths at the expense of latency [131].

Frequency interleaver For MIMO scheme, both MIMO branches, i.e. the signal generation for both transmit antennas, shall use the same frequency interleaver configuration. In addition, since MIMO services can be multiplexed with MISO services into one NGH frame, pair-wise interleaving scheme is necessary. As described in [44], this latter condition comes from the use of Alamouti encoding for MISO in NGH system as Alamouti-encoded output pair cells should experience similar channel realizations to avoid the loss of the code orthogonality. In OFDM system, the cells are mapped to frequency carriers, hence, to output of the Alamouti code is mapped to adjacent carriers to maximize the probability of similar channel realizations. For simplicity, NGH system re-uses the frequency interleaving scheme already adopted for SISO transmission. That is, the frequency interleaver in a given FFT mode only generates half of the interleaver addresses compared to the addresses generated in SISO transmissions. The cells pair always shares the address and interleaved together into adjacent carrier positions.

The frequency interleaver adopted in ATSC 3.0 has similar structure to the DVB-NGH frequency interleaver and is described in [132].

Pilot Paterns For MIMO orthogonal pilot patterns among the transmit antennas allow at the receiver estimation of the channel response from each transmit antenna. DVB-NGH inherits the pilot patten scheme from MISO DVB-T2 which is based on the Walsh-Hadamard encoding algorithm [17, 133]. With Walsh-Hadamard MIMO pilot encoding, the pilots from the second transmit antenna are inverted compared to the first transmit antenna on alternate scattered pilot bearing carriers. Both antennas shall transmit in all pilot positions. In the case of intentional transmit power imbalance between transmit antennas, the power of the pilots has to be modified accordingly to have the same power imbalance as the data. This is because the transmit power imbalance 
is included as part of the precoder (cf. Section 3.2.3) affecting only the data carriers.

ATSC 3.0 in addition to the Walsh-Hadamard encoding algorithm, it also includes null pilots encoding algorithm. However only one pilot algorithm may be used in a given frame. With MIMO null pilot encoding, in opposition to Walsh-Hadamard encoding, two orthogonal pilot patterns are defined where each pattern does not share common pilot carrier positions with the other. Here, the pilots in each transmit antenna have $3 \mathrm{~dB}$ increased transmit power to compensate for the null pilots not transmitted in the other group.

The null pilots encoding might be more suited for static channel conditions (e.g., for fixed roof-top reception) while the Walsh-Hadamard pilot encoding might be more suited for mobile channels. The reason is that the pilot spacing in time direction is higher for the null pilots encoding compared to a pilot spacing of the Walsh-Hadamard pilot encoding. For fast varying channel conditions, it is more challenging for the channel estimation to follow these channel variations if the pilot spacing in time direction is increased.

\section{Waveform}

The OFDM modulator is the same as for the SISO profile, i.e. IFFT operation plus guard interval insertion. For the MIMO rate-2 profile it is duplicated to process the information of each transmit layer. The same IFFT operation process is applied for DVB-NGH and ATSC 3.0. 
APPENDIX B. DVB-NGH AND ATSC 3.0 MIMO PHYSICAL

LAYERS 


\section{References}

[1] Frame structure channel coding and modulation for a second generation digital terrestrial television broadcasting system (DVB-T2), ETSI Std. EN 302 755, Rev. 1.2.1, 2011.

[2] "DVB-T2 - 2nd Generation Terrestrial Broadcasting," DVB Fact Sheet, 2015.

[3] J. Doeven, P. Walop, E. Zilles, J.-J. Guitot, and S. Ripley, "Digital Dividend: Insights for Spectrum Decisions," ITU Technical Report, 2012.

[4] "Cisco Visual Networking Index: Global Mobile Data Traffic Forecast Update, 2015-2020," White Paper, CISCO, 2016.

[5] W. Zhang, Y. Wu, N. Hur, T. Ikeda, and P. Xia, "FOBTV: Worldwide Efforts in Developing Next-Generation Broadcasting System," IEEE Transactions on Broadcasting, vol. 60, no. 2, pp. 154-159, June 2014.

[6] M. Simon and M. A. Aitken, "An Overview of the North American ATSC M/H Mobile Broadcasting System and Its Next-Generation ATSC 3.0," in Next Generation Mobile Broadcasting, D. Gomez-Barquero, Ed. Boca Raton, FL, USA: CRC Press, 2013, pp. 91-122.

[7] T. Masayuki et al., "An Overview of the ISDB-T One-Seg Broadcasting, ISDB-TSB and ISDB-Tmm," in Next Generation Mobile Broadcasting, D. Gomez-Barquero, Ed. Boca Raton, FL, USA: CRC Press, 2013, pp. $31-68$.

[8] Y. Zhang, W. Guan and D. He, "Overview of the Chinese Digital Terrestrial Multimedia Broadcasting System," in Next Generation Mobile Broadcasting, D. Gomez-Barquero, Ed. Boca Raton, FL, USA: CRC Press, 2013, pp. 123-150. 


\section{REFERENCES}

[9] D. Gomez-Barquero, "An Overview of the Next-Generation Mobile Digital Video Broadcasting Standard DVB-NGH," in Next Generation Mobile Broadcasting, D. Gomez-Barquero, Ed. Boca Raton, FL, USA: CRC Press, 2013, pp. 186-219.

[10] R. Gallager, "Low-density parity-check codes," IRE Transactions on Information Theory, vol. 8, no. 1, pp. 21-28, Jan. 1962.

[11] K. J. Kim et al., "Low-Density Parity-Check Codes for ATSC 3.0," IEEE Transactions on Broadcasting, vol. 62, no. 1, pp. 189-196, March 2016.

[12] B. Bross, "Overview of the HEVC Video Coding Standard," in Next Generation Mobile Broadcasting, D. Gomez-Barquero, Ed. Boca Raton, FL, USA: CRC Press, 2013, pp. 713-748.

[13] D. Tse and P. Viswanath, Fundamentals of Wireless Communications. Cambridge University Press, 2005.

[14] D. Vargas, D. Gozalvez, D. Gomez-Barquero, and N. Cardona, "MIMO for DVB-NGH, the Next Generation Mobile TV Broadcasting," IEEE Communications Magazine, vol. 51, no. 7, pp. 130-137, July 2013.

[15] D. Gomez-Barquero, D. Vargas, M. Fuentes, P. Klenner, S. Moon, J. Y. Choi, S. Schneider, and K. Murayama, "MIMO for ATSC 3.0," IEEE Transactions on Broadcasting, vol. 62, no. 1, pp. 298-305, March 2016.

[16] S. Saito et al., "8K Terrestrial Transmission Field Tests Using DualPolarized MIMO and Higher-Order Modulation OFDM," IEEE Transactions on Broadcasting, vol. 62, no. 1, pp. 306-315, March 2016.

[17] P. Moss and T. Y. Poon, "Overview of the Multiple-Input MultipleOutput Terrestrial Profile of DVB-NGH," in Next Generation Mobile Broadcasting, D. Gomez-Barquero, Ed. Boca Raton, FL, USA: CRC Press, 2013, pp. 549-580.

[18] A. J. Paulraj, D. A. Gore, R. U. Nabar, and H. Bolcskei, "An overview of MIMO communications - a key to gigabit wireless," Proceedings of the IEEE, vol. 92, no. 2, pp. 198-218, Feb. 2004.

[19] R. Monnier, J. Rault, and T. De Couasnon, "Digital Television Broadcasting with High Spectral Efficiency," in International Broadcasting Convention, IBC, July 1992, pp. 380-384.

[20] J. Mitchell, P. Moss, and M. Thorp, "A dual polarisation MIMO broadcast TV System," White Paper, British Broadcasting Corporation, 2006. 
[21] J. Boyer, P. G. Brown, K. Hayler, M. L. Garcia, J. D. Mitchell, P. N. Moss, and M. J. Thorp, "MIMO for Broadcast-Results from a High-Power UK Trial," White Paper, British Broadcasting Corporation, 2007.

[22] P. Moss, "2-by-2 MIMO Fixed Reception Channel Model for Dual-Polar Terrestrial Transmission," White Paper, British Broadcasting Corporation, 2008.

[23] T. Shitomi, K. Murayama, M. Taguchi, S. Asakura, and K. Shibuya, "Technology for next-generation digital terrestrial broadcasting - Field experiments of dual-polarized MIMO-OFDM transmission using LDPC codes," in IEEE International Symposium on Broadband Multimedia Systems and Broadcasting (BMSB), June 2012, pp. 1-6.

[24] Y. Narikiyo, M. Nakamura, H. Sanei, and M. Takada, "Mobile reception performance of sfbc-mimo transmission system with walsh code scattered pilot - computer simulation and field test results," in IEEE International Symposium on Broadband Multimedia Systems and Broadcasting (BMSB), June 2013, pp. 1-5.

[25] T. Shitomi, K. Murayama, S. Asakura, and K. Shibuya, "A study on advanced single frequency network technology using STC-SDM transmission," in IEEE International Symposium onBroadband Multimedia Systems and Broadcasting (BMSB), June 2013, pp. 1-5.

[26] S. M. Alamouti, "A simple transmit diversity technique for wireless communications," IEEE J. Sel. Areas Comm., vol. 16, no. 10, pp. 1451-1458, Oct. 1998.

[27] J. Robert, "Terrestrial TV Broadcast Using Multi-Antenna Systems," Ph.D. dissertation, Technical University Braunschweig, 2013.

[28] M. Slimani, J. Robert, P. Schlegel, U. Reimers, R. Burow, F. Kattanek, R. Pfeffer, and L. Stadelmeier, "Results of the DVB-T2 Field Trial in Germany," IEEE Transactions on Broadcasting, vol. 61, no. 2, pp. 177194, June 2015.

[29] D. Gozalvez, "Combined Time, Frequency and Space Diversity in Multimedia Mobile Broadcasting Systems," Ph.D. dissertation, Universitat Politecnica de Valencia, 2012.

[30] H. Miyasaka et al., "A study on MIMO OFDM transmissions using interpolarization spreading," in Broadband Multimedia Systems and Broadcasting (BMSB), 2015 IEEE International Symposium on, June 2015, pp. $1-4$. 


\section{REFERENCES}

[31] T. Jokela, C. Hollanti, J. Lahtonen, R. Vehkalahti, and J. Paavola, "Performance Evaluation of $4 \times 2$ MIMO Schemes for Mobile Broadcasting," in IEEE International Symposium on Broadband Multimedia Systems and Broadcasting (BMSB), June 2011, pp. 1-6.

[32] T. Jokela, P.-D. Arapoglou, C. Hollanti, M. Bhavani Shankar, and V. Tapio, "Hybrid SatelliteTerrestrial MIMO for Mobile Digital Broadcasting," in Next Generation Mobile Broadcasting, D. Gomez-Barquero, Ed. Boca Raton, FL, USA: CRC Press, 2013, pp. 713-748.

[33] Y. Nasser, J.-F. Helard, and M. Crussiere, "3D MIMO Scheme for Broadcasting Future Digital TV in Single-Frequency Networks," Electronics Letters, vol. 44, no. 13, pp. 829-830, June 2008.

[34] M. Liu, M. Helard, M. Crussiere, and J.-F. Helard, "Distributed MIMO coding Scheme with Low Decoding Complexity for Future Mobile TV Broadcasting," Electronics Letters, vol. 48, no. 17, pp. 1079-1081, Aug. 2012.

[35] M. Liu, J.-F. Helard, M. Helard, and M. Crussiere, "Towards the Next Generation Video Broadcasting: Improved Performance Using Distributed MIMO," Wireless Personal Communications, May 2015.

[36] S. Sesia, I. Toufik, and M. Baker, LTE - The UMTS Long Term Evolution: From Theory to Practice, 2nd ed. Chichester, UK: John Wiley and Sons, Ltd., 2011.

[37] G. Caire, G. Taricco, and E. Biglieri, "Bit-interleaved coded modulation," IEEE Transactions on Information Theory, vol. 44, no. 3, pp. 927-946, May 1998.

[38] A. Guillen i Fabregas, A. Martinez, and G. Caire, "Bit-interleaved coded modulation," Foundations and Trends in Communications and Information Theory, vol. 5, no. 1-2, pp. 1-153, 2008.

[39] G. Ungerboeck, "Channel coding with multilevel/phase signals," IEEE Transactions on Information Theory, vol. 28, no. 1, pp. 55-67, Jan. 1982.

[40] U. Wachsmann, R. Fischer, and J. Huber, "Multilevel codes: theoretical concepts and practical design rules," IEEE Transactions on Information Theory, vol. 45, no. 5, pp. 1361-1391, July 1999.

[41] C. Studer and H. Bolcskei, "Soft Input Soft Output Single Tree-Search Sphere Decoding," IEEE Transactions on Information Theory, vol. 56, no. 10 , pp. $4827-4842$, Oct. 2010. 
[42] I. Sobron, "Detection and Decoding Algorithms of Multi-Antenna Diversity Techniques for Terrestrial DVB Systems," Ph.D. dissertation, Mondragon Unibertsitatea, 2010.

[43] M. Witzke, S. Baro, F. Schreckenbach, and J. Hagenauer, "Iterative detection of MIMO signals with linear detectors," in Thirty-Sixth Asilomar Conference on Signals, Systems and Computers, vol. 1, Nov. 2002, pp. 289-293.

[44] D. Vargas, S. Moon, W.-S. Ko, and D. Gomez-Barquero, "Enhanced MIMO Spatial Multiplexing with Phase Hopping for DVB-NGH," in Next Generation Mobile Broadcasting, D. Gomez-Barquero, Ed. Boca Raton, FL, USA: CRC Press, 2013, pp. 609-634.

[45] Implementation guidelines for a second generation digital terrestrial television broadcasting system (DVB-T2), ETSI Std. TR 102 831, Rev. $0.10 .4,2010$.

[46] D. Gozalvez, D. Gomez-Barquero, D. Vargas, and N. Cardona, "Time Diversity in Mobile DVB-T2 Systems," IEEE Transactions on Broadcasting, vol. 57, no. 3, pp. 617-628, Sept. 2011.

[47] P. Gomez, D. Gomez-Barquero, D. Gozalvez, A. Añorga, and M. Breiling, "Time Interleaving in DVB-NGH," in Next Generation Mobile Broadcasting, D. Gomez-Barquero, Ed. Boca Raton, FL, USA: CRC Press, 2013, pp. 609-634.

[48] H. Sampath and A. Paulraj, "Linear Precoding for Space-Time Coded Systems with Known Fading Correlations," IEEE Communications Letters, vol. 6, no. 6, pp. 239-241, June 2002.

[49] S. Zhou and G. Giannakis, "Optimal Transmitter Eigen-Beamforming and Space-Time Block Coding Based on Channel Mean Feedback," IEEE Transactions on Signal Processing, vol. 50, no. 10, pp. 2599-2613, Oct. 2002.

[50] M. Vu and A. Paulraj, "MIMO Wireless Linear Precoding," IEEE Signal Processing Magazine, vol. 24, no. 5, pp. 86-105, Oct. 2007.

[51] D. Love, R. Heath, V. K. N. Lau, D. Gesbert, B. Rao, and M. Andrews, "An Overview of Limited Feedback in Wireless Communication Systems," IEEE Journal on Selected Areas in Communications, vol. 26, no. 8, pp. 1341-1365, Oct. 2008.

[52] S. Rosati, S. Tomasin, M. Butussi, and B. Rimoldi, "LLR Compression for BICM Systems Using Large Constellations," IEEE Transactions on Communications, vol. 61, no. 7, pp. 2864-2875, July 2013. 


\section{REFERENCES}

[53] M. C. Jeruchim, P. Balaban, and K. S. Shanmugan, Simulation of Communication Systems, Modeling, Methodology, and Techniques. Springer US, 2002.

[54] C. E. Shannon, "A mathematical theory of communication," Bell Syst. Tech. J, vol. 27, pp. 379-423, 1948.

[55] S. R. Saunders and A. Aragón-Zavala, Antennas and Propagation for Wireless Communication Systems, 2nd ed., J. . S. Ltd, Ed., 2007.

[56] E. Telatar, "Capacity of multi-antenna Gaussian channels," European Trans. Telecomm., vol. 10, no. 6, pp. 585-596, Nov. 1999.

[57] G. Strang, Introduction to Linear Algebra, 4th ed. Wellesley-Cambridge Press, 2009.

[58] L. Zheng and D. Tse, "Diversity and Multiplexing: a Fundamental Tradeoff in Multiple-Antenna Channels," IEEE Transactions on Information Theory, vol. 49, no. 5, pp. 1073-1096, May 2003.

[59] E. Biglieri, R. Calderbank, A. Constantinides, A. Goldsmith, A. Paulraj, and P. H. V., Eds., MIMO Wireless Communications. Cambridge University Press, 2007.

[60] J. Robert and J. Zoellner, "Multiple-Input Single-Output Antenna Schemes for DVB-NGH," in Next Generation Mobile Broadcasting, D. Gomez-Barquero, Ed. Boca Raton, FL, USA: CRC Press, 2013, pp. $581-608$.

[61] S. LoPresto, R. Citta, D. Vargas, and D. Gómez-Barquero, "Transmit Diversity Code Filter Sets (TDCFS), a MISO Antenna Frequency PreDistortion Scheme for ATSC 3.0," IEEE Transactions on Broadcasting, vol. 62, no. 1, pp. 271-280, March 2016.

[62] J.-C. Belfiore, G. Rekaya, and E. Viterbo, "The golden code: a $2 \times$ 2 Full-Rate Space-Time Code with Nonvanishing Determinants," IEEE Transactions on Information Theory, vol. 51, no. 4, pp. 1432-1436, April 2005.

[63] S. Moon, W.-S. Ko, D. Vargas, D. Gozalvez, M. Nisar, and V. Pauli, "Enhanced Spatial Multiplexing for Rate-2 MIMO of DVB-NGH System," in Proccedings of 19th International Conference on Telecommunications (ICT), 2012, April 2012, pp. 1-5.

[64] H. Jafarkhani, "A Quasi-Orthogonal Space-Time Block Code," IEEE Transactions on Communications, vol. 49, no. 1, pp. 1-4, Jan. 2001. 
[65] C. Hollanti, J. Lahtonen, and H. feng L., "Maximal Orders in the Design of Dense Space-Time Lattice Codes," IEEE Transactions on Information Theory, vol. 54, no. 10, pp. 4493-4510, Oct. 2008.

[66] R. Vehkalahti and C. Hollanti, "Reducing Complexity with Less Than Minimum Delay Space-Time Lattice Codes," in IEEE Information Theory Workshop, Oct. 2011, pp. 130-134.

[67] C. Hollanti, R. Vehkalahti, and Y. Nasser, "Algebraic Hybrid SatelliteTerrestrial Space-Time Codes for Digital Broadcasting in SFN," in IEEE Workshop on Signal Processing Systems (SiPS), Oct. 2011, pp. 234-238.

[68] C. Berrou, A. Glavieux, and P. Thitimajshima, "Near Shannon limit error-correcting coding and decoding: Turbo-codes," in IEEE International Conference on Communications, vol. 2, Geneva, Switzerland, May 1993, pp. 1064-1070.

[69] D. MacKay, "Good error-correcting codes based on very sparse matrices," IEEE Transactions on Information Theory, vol. 45, no. 2, pp. 399-431, March 1999.

[70] D. Costello Jr. and G. Forney Jr., "Channel coding: The road to channel capacity," Proceedings of the IEEE, vol. 95, no. 6, pp. 1150-1177, June 2007.

[71] D. Gomez-Barquero, Next Generation Mobile Broadcasting. CRC Press, 2013.

[72] F. Kschischang, B. Frey, and H.-A. Loeliger, "Factor graphs and the sumproduct algorithm," IEEE Transactions on Information Theory, vol. 47, no. 2, pp. 498-519, Feb. 2001.

[73] T. Richardson and R. Urbanke, "The capacity of low-density parity-check codes under message-passing decoding," IEEE Transactions on Information Theory, vol. 47, no. 2, pp. 599-618, Feb. 2001.

[74] G. Foschini, R. Gitlin, and S. Weinstein, "Optimization of twodimensional signal constellations in the presence of gaussian noise," IEEE Transactions on Communications, vol. 22, no. 1, pp. 28-38, Jan. 1974.

[75] J. Stott, "CM and BICM limits for rectangular constellations," White Paper, British Broadcasting Corporation, August 2013.

[76] J. Zoellner and N. Loghin, "Optimization of high-order non-uniform qam constellations," in IEEE International Symposium on Broadband Multimedia Systems and Broadcasting (BMSB), June 2013, pp. 1-6. 
[77] D. Gomez-Barquero, C. Douillard, P. Moss, and V. Mignone, "DVBNGH: The Next Generation of Digital Broadcast Services to Handheld Devices," IEEE Transactions on Broadcasting, vol. 60, no. 2, pp. 246-257, June 2014.

[78] L. Michael and D. Gomez-Barquero, "Bit-Interleaved Coded Modulation (BICM) for ATSC 3.0," IEEE Transactions on Broadcasting, vol. 62, no. 1, pp. 181-188, March 2016.

[79] P. Xue, K. Bae, K. Kim, and H. Yang, "M-best candidates based softdemapper for rotated M-QAM constellation," in IEEE 11th Consumer Communications and Networking Conference (CCNC), Jan. 2014, pp. 623-628.

[80] M. Fuentes, D. Vargas, and D. Gomez-Barquero, "Low-Complexity Demapping Algorithm for Two-Dimensional Non-Uniform Constellations," IEEE Transactions on Broadcasting, pp. 1-9, 2016.

[81] T. Shitomi, S. Asakura, S. Saito, T. Saito, and K. Shibuya, "Study on circularly arranged non-uniform constellations in dual-polarized MIMOOFDM transmission," in IEEE International Symposium on Broadband Multimedia Systems and Broadcasting (BMSB), June 2014, pp. 1-4.

[82] L. Szczecinski and A. Alvarado, Bit-Interleaved Coded Modulation: Fundamentals, Analysis and Design. Wiley, Feb. 2015.

[83] A. Chindapol and J. Ritcey, "Design, analysis, and performance evaluation for BICM-ID with square QAM constellations in Rayleigh fading channels," IEEE Journal on Selected Areas in Communications, vol. 19, no. 5, pp. 944-957, May 2001.

[84] M. Davey and D. MacKay, "Low-density parity check codes over GF(q)," IEEE Communications Letters, vol. 2, no. 6, pp. 165-167, June 1998.

[85] E. Boutillon, L. Conde-Canencia, and A. Al Ghouwayel, "Design of a GF(64)-LDPC Decoder Based on the EMS Algorithm," IEEE Transactions on Circuits and Systems I: Regular Papers, vol. 60, no. 10, pp. 2644-2656, Oct. 2013.

[86] B. Hochwald and S. ten Brink, "Achieving near-capacity on a multipleantenna channel," IEEE Transactions on Communications, vol. 51, no. 3, pp. 389-399, March 2003.

[87] C. Douillard, C. B. Michel Jezequel, A. Picart, P. Didier, and A. Glavieux, "Iterative correction of intersymbol interference: Turbo-equalisation," European Trans. Telecomm., vol. 6, no. 5, pp. 507-511, Sept./Oct. 1995. 
[88] R. Koetter, A. Singer, and M. Tuchler, "Turbo equalization," IEEE Signal Processing Magazine, vol. 21, no. 1, pp. 67-80, Jan. 2004.

[89] D. Vargas, A. Winkelbauer, G. Matz, D. Gomez-Barquero, and N. Cardona, "Low complexity iterative MIMO receivers for DVB-NGH using soft MMSE demapping and quantized log-likelihood ratios," in Proceedings of the COST IC1004, Barcelona, Spain, Feb. 2012.

[90] D. Seethaler, G. Matz, and F. Hlawatsch, "An efficient MMSE-based demodulator for MIMO bit-interleaved coded modulation," in IEEE Global Telecommunications Conference, vol. 4, Nov. 2004, pp. 2455-2459.

[91] L. Barbero and J. Thompson, "Extending a fixed-complexity sphere decoder to obtain likelihood information for turbo-MIMO systems," IEEE Transactions on Vehicular Technology, vol. 57, no. 5, pp. 2804-2814, Sept. 2008.

[92] P. Fertl, J. Jalden, and G. Matz, "Performance assessment of MIMOBICM demodulators based on mutual information," IEEE Transactions on Signal Processing, vol. 60, no. 3, pp. 1366-1382, March 2012.

[93] M. Danieli, S. Forchhammer, J. Andersen, L. Christensen, and S. Christensen, "Maximum mutual information vector quantization of loglikelihood ratios for memory efficient HARQ implementations," in Proc. Data Compression Conference (DCC 2010), March 2010, pp. 30-39.

[94] A. Winkelbauer, G. Matz, and A. Burg, "Channel-optimized vector quantization with mutual information as fidelity criterion," in Asilomar Conference on Signals, Systems and Computers, Nov. 2013, pp. 851-855.

[95] A. Winkelbauer, "Blind Performance Estimation and Quantizer Design with Applications to Relay Networks," Ph.D. dissertation, Institute of Telecommunications, Vienna University of Technology, 2014.

[96] D. Vargas, Y. J. D. Kim, J. Bajcsy, D. Gomez-Barquero, and N. Cardona, "A MIMO-Channel-Precoding Scheme for Next Generation Broadcast TV Systems," IEEE Transactions on Broadcasting, vol. 61, no. 3, pp. 445-456, Sept. 2015.

[97] G. H. Golub and C. F. Van Loan, Matrix Computations, 3rd ed. Baltimore: Johns Hopkins University Press, 1996.

[98] S. P. Boyd and L. Vandenberghe, Convex Optimization. Cambridge University Press, 2004. 


\section{REFERENCES}

[99] E. Jorswieck and H. Boche, "Behavior of outage probability in MISO systems with no channel state information at the transmitter," in Proceedings IEEE Information Theory Workshop, March 2003, pp. 353-356.

[100] B. Varadarajan and J. Barry, "The outage capacity of linear space-time codes," IEEE Transactions on Wireless Communications, vol. 4, no. 6, pp. 2642-2648, Nov. 2005.

[101] J.-S. Baek and J.-S. Seo, "Efficient Pilot Patterns and Channel Estimations for MIMO-OFDM Systems," IEEE Transactions on Broadcasting, vol. 58, no. 4, pp. 648-653, Dec. 2012.

[102] T. M. Cover and J. A. Thomas, Elements of Information Theory. New York: Wiley, 1991.

[103] M. Vu and A. Paulraj, "On the capacity of MIMO Wireless Channels with Dynamic CSIT," IEEE Journal on Selected Areas in Communications, vol. 25, no. 7, pp. 1269-1283, Sept. 2007.

[104] R. Kobeissi, S. Sezginer, and F. Buda, "Downlink Performance Analysis of Full-Rate STCs in 2x2 MIMO WiMAX Systems," in Proceedings IEEE Vehicular Technology Conference (VTC), April 2009, pp. 1-5.

[105] B. Roslind Jose, "Design Techniques For Sigma-Delta Based ADC For Wireless Applications," Ph.D. dissertation, Cochin University Of Science And Technology, 2010.

[106] J. Max, "Quantizing for minimum distortion," IRE Transactions on Information Theory, vol. 6, no. 1, pp. 7-12, March 1960.

[107] S. Lloyd, "Least squares quantization in PCM," IEEE Transactions on Information Theory, vol. 28, no. 2, pp. 129-137, March 1982.

[108] S. Roger, A. Gonzalez, V. Almenar, and G. Matz, "An Efficient FixedComplexity Sphere Decoder with Quantized Soft Outputs," IEEE Communications Letters, vol. 16, no. 11, pp. 1828-1831, Nov. 2012.

[109] C. Novak, "Design of multiuser and multi-antenna communication systems based on graphical models and soft information," Ph.D. dissertation, Institute of Telecommunications, Vienna University of Technology, 2010 .

[110] A. Winkelbauer and G. Matz, "On quantization of log-likelihood ratios for maximum mutual information," in IEEE 16th International Workshop on Signal Processing Advances in Wireless Communications (SPAWC), June 2015, pp. 316-320. 
[111] N. Tishby, F. Pereira, and W. Bialek, "The information bottleneck method," in Proc. 37th Allerton Conf. on Communication, Control, and Computing, Sept. 1999, pp. 368-377.

[112] A. Winkelbauer and G. Matz, "Joint network-channel coding for the asymmetric multiple-access relay channel," in Proc. IEEE ICC 2012, June 2012.

[113] J. J. Giménez, "Improved Spectrum Usage with Multi-RF Channel Aggregation Technologies for the Next-Generation Terrestrial Broadcasting," Ph.D. dissertation, Universitat Politecnica de Valencia, 2015.

[114] J. Montalban, "Solutions for new terrestrial broadcasting systems offering simultaneously stationary and mobile services," Ph.D. dissertation, Universidad del Pas Vasco/Euskal Herriko Unibertsitatea, 2014.

[115] L. Zhang et al., "Layered-Division-Multiplexing: Theory and Practice," IEEE Transactions on Broadcasting, vol. 62, no. 1, pp. 216-232, March 2016.

[116] S. Jeon, J. Han, R. Shrestha, S. Park, H. Mok, H. Kim, and J. Seo, "MIMO Cloud Transmission Based on BICM-ID for High Data Rate Local Contents Delivery," IEEE Transactions on Broadcasting, vol. 61, no. 4 , pp. 580-589, Dec. 2015.

[117] N. Loghin, J. Zoellner, B. Mouhouche, D. Ansorregui, J. Kim, and S. Park, "Non-Uniform Constellations for ATSC 3.0," IEEE Transactions on Broadcasting, vol. 62, no. 1, pp. 197-203, March 2016.

[118] S. Roger, "Design and Implementation of Efficient Algorithms for Wireless MIMO Communication Systems," Ph.D. dissertation, Universitat Politecnica de Valencia, 2012.

[119] C. Studer, "Iterative MIMO Decoding: Algorithms and VLSI Implementation Aspects," Ph.D. dissertation, ETH Zurich, 2009.

[120] B. M. Kurkoski and H. Yagi, "Quantization of Binary-Input Discrete Memoryless Channels," IEEE Transactions on Information Theory, vol. 60, no. 8, pp. 4544-4552, Aug. 2014.

[121] F. Quitin, "Channel modeling for polarized MIMO systems," Ph.D. dissertation, Université Libre de Bruxelles/Université catholique de Louvain, 2011.

[122] P. Moss, "MIMO Technology in Broadcasting - and an Application in Programme-Making," in 2nd IEEE Broadcast Technology Society Gold Workshop on Next Generation Broadcasting, Cagliari, March 2013. 
[123] — "SM-MIMO Channel Models," DVB Document TM-MIMO0051, 2013.

[124] P. Moss, T. Y. Poon, and J. Boyer, "A simple model of the UHF crosspolar terrestrial channel for DVB-NGH," White Paper, British Broadcasting Corporation, 2011.

[125] M. Patzold, U. Killat, F. Laue, and Y. Li, "On the statistical properties of deterministic simulation models for mobile fading channels," IEEE Transactions on Vehicular Technology, vol. 47, no. 1, pp. 254-269, Feb. 1998.

[126] X. Zhang and X. Zhou, LTE-Advanced Air Interface Technology. CRC Press, 2012.

[127] N. Czink, "The random-cluster model - a stochastic MIMO channel model for broadband communication systems of the 3rd generation and beyond," Ph.D. dissertation, Institut für Nachrichtentechnik und Hochfrequenztechnik, Vienna University of Technology, 2007.

[128] L. Jiang, V. Jungnickel, S. Jaeckel, L. Thiele, and A. Brylka, "Correlation analysis of multiple-input multiple-output channels with cross-polarized antennas," in 14th Asia-Pacific Conference on Communications, 2008. APCC 2008, Oct. 2008, pp. 1-5.

[129] LTE; Evolved Universal Terrestrial Radio Access (E-UTRA); Base Station (BS) radio transmission and reception, ETSI Std. TS 136 104, Rev. 0.11.0, 2013.

[130] R. Nabar, H. Bolcskei, V. Erceg, D. Gesbert, and A. Paulraj, "Performance of Multiantenna Signaling Techniques in the Presence of Polarization Diversity," IEEE Transactions on Signal Processing, vol. 50, no. 10, pp. 2553-2562, Oct. 2002.

[131] P. Klenner, J. S. Baek, N. S. Loghin, D. Gomez-Barquero, and W. S. Ko, "Physical Layer Time Interleaving for the ATSC 3.0 System," IEEE Transactions on Broadcasting, vol. 62, no. 1, pp. 253-262, March 2016.

[132] M. Earnshaw, K. Shelby, H. Lee, Y. Oh, and M. Simon, "Physical Layer Framing for ATSC 3.0," IEEE Transactions on Broadcasting, vol. 62, no. 1, pp. 263-270, March 2016.

[133] J. Morgade, P. Angueira, A. Arrinda, R. Pfeffer, V. Steinmann, J. Frank, and R. Brugger, "SFN-SISO and SFN-MISO Gain Performance Analysis for DVB-T2 Network Planning," IEEE Transactions on Broadcasting, vol. 60, no. 2, pp. 272-286, June 2014. 